Copyright

by

Merve Celen

2016 
The Dissertation Committee for Merve Celen certifies that this is the approved version of the following dissertation:

\section{Joint Maintenance and Production Operations Decision Making in Flexible Manufacturing Systems}

\section{Committee:}

Dragan Djurdjanovic, Supervisor

F. Frank Chen

John J. Hasenbein

Erhan Kutanoglu

David P. Morton 


\title{
Joint Maintenance and Production Operations Decision Making in Flexible Manufacturing Systems
}

by

\section{Merve Celen, B.S.; M.S.}

\author{
Dissertation \\ Presented to the Faculty of the Graduate School of \\ The University of Texas at Austin \\ in Partial Fulfillment \\ of the Requirements \\ for the Degree of \\ Doctor of Philosophy
}

The University of Texas at Austin

August 2016 


\section{Acknowledgements}

First and above all, I would like to express my gratitude to my supervisor, Dr. Dragan Djurdjanovic. He always knew when to guide me and when to leave me to find my own way, when to push me and when to let me take a break. This dissertation would not have been possible without his full support and trust in me. Next, I would like to thank my committee members, who were mentors or instructors at various points in my Ph.D. work. The guidance they provided in coursework, one-on-one meetings as well as in informal conversations was invaluable. I also would like to thank Emeritus Prof. Barnes for introducing me to Metaheuristics and sharing my excitement in utilizing them in my work.

I would like to thank my colleagues who have been in the Limes Lab at any point during my studies: Alex Bleakie, Kevin Wang, Marcus Musselman, Michael Cholette, Yibo Jiao, Asad U1 Haq, Matthew Graves, Derek Zhang and Yi Liu.

I would also like to give my special thanks to my parents and sister for their love and support in spite of being so far away.

Lastly, I would like to thank my husband, David Johnson. His constant love and encouragement gave me the strength to overcome the challenges I faced during my studies and cheered me up. 


\title{
Joint Maintenance and Production Operations Decision Making in Flexible Manufacturing Systems
}

\author{
Merve Celen, Ph.D. \\ The University of Texas at Austin, 2016
}

Supervisor: Dragan Djurdjanovic

\begin{abstract}
In highly flexible and highly integrated manufacturing systems such as semiconductor manufacturing, equipment has the capability of conducting different manufacturing operations and/or producing at various speeds. In such systems, degradation of a machine depends highly on the operations performed on it. Selection of operations executed on an equipment changes the degradation dynamics and hence directly affects preventive maintenance (PM) decisions. On the other hand, PM actions interrupt production and change the system reliability and equipment availability, which in turn directly affects decisions as to which operations should be performed on which piece of equipment. These strong dynamic interactions between equipment condition, operations executed on the equipment and product quality necessitate a methodology that integrates the decisions of maintenance scheduling and production operations. Currently, maintenance and production operations decision-making are two decoupled processes. To address the aforementioned problems, in this dissertation, we devise integrated decision-
\end{abstract}


making policies for maintenance scheduling and production operations in flexible manufacturing systems (FMS) optimizing a customizable objective function that takes into account operation-dependent degradation models and production targets. The objective function consists of costs associated with scheduled and unscheduled maintenance, rewards for successfully completed products and penalties for missed production targets. In order to maximize the objective function, a paradigm based on metaheuristic optimization and evaluation of candidate solutions via discrete-event simulations of operations of the underlying manufacturing system is used. Firstly, we propose an operation-dependent decision-making policy for a multiple-product/multipleequipment manufacturing system, where each product requires several operations for completion and the sequence in which different product types are produced is a priori given. The proposed method is tested in simulations of a cluster tool and the results show that operation-dependent maintenance decision-making outperforms the case where maintenance decisions are made without considerations of operation-dependent degradation dynamics. Secondly, we propose an integrated decision-making policy for maintenance scheduling and product sequencing where the sequence in which different product types can be arranged in a way to maximize the customizable profit function. The results show that jointly making maintenance and production sequencing decisions consistently and often significantly outperforms the current practice of making these decisions separately. Finally, a joint maintenance scheduling and production operations decision making policy is proposed for a flexible manufacturing system where the 
degradation states of the equipment are not perfectly observable, but are rather hidden states of a known Hidden Markov Model (HMM). Proposed integrated decision-making policy under imperfect degradation state observations is shown to consistently outperform the benchmark policies. 


\section{Table of Contents}

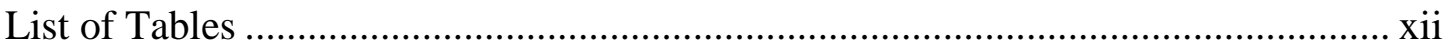

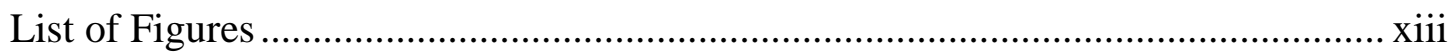

Chapter 1 .................................................................................................1

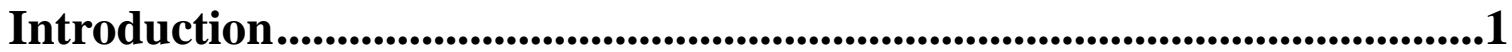

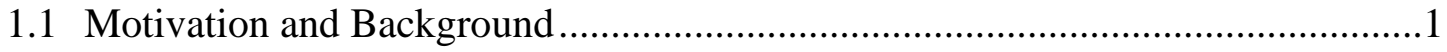

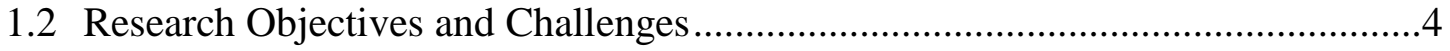

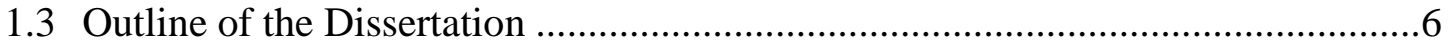

Chapter 2 ......................................................................................................7

Literature Review ...............................................................................................7

2.1 Maintenance Strategies in Manufacturing ...........................................................

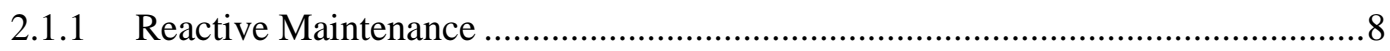

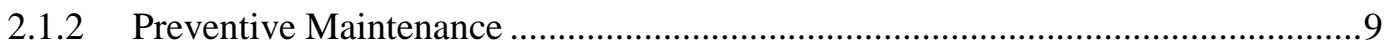

2.1.2.1 Reliability Based Maintenance ........................................................... 10

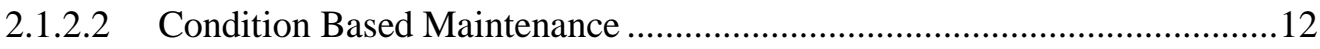

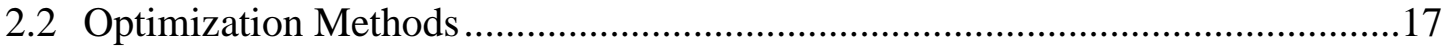

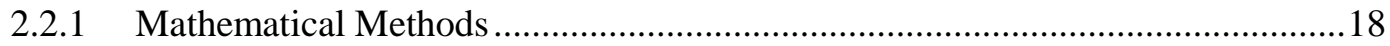

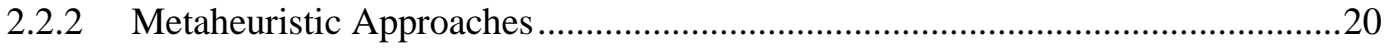

2.2.3 Simulation Based Optimization........................................................................24

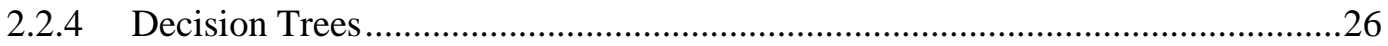

2.3 Joint Scheduling of Maintenance and Production Operations ...........................26

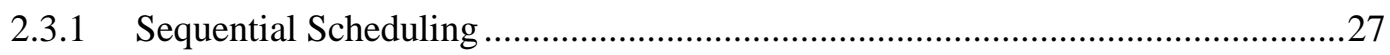

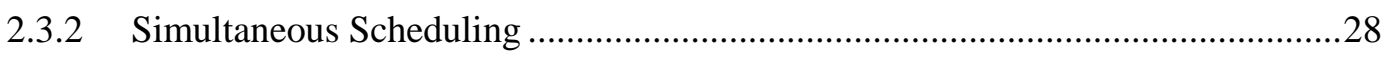

Chapter 3 ................................................................................................................ 34

\section{Operation-Dependent Maintenance Scheduling in Flexible} Manufacturing Systems........................................................................................ 34 


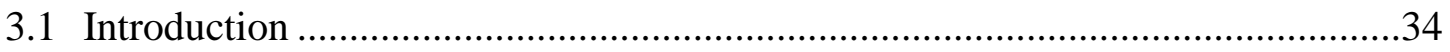

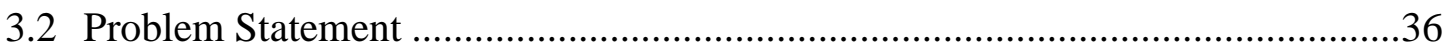

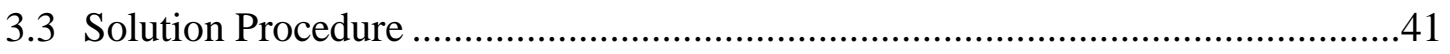

3.3.1 Operation-Dependent Maintenance Decision-Making .......................................41

3.3.1.1 Solution Representation ................................................................... 41

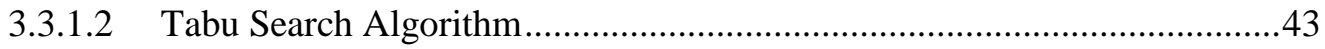

3.3.2 Operation-Independent CBM Policy Determination ..........................................46

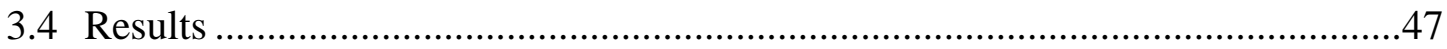

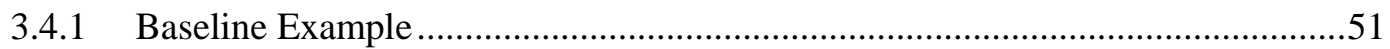

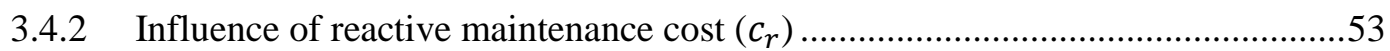

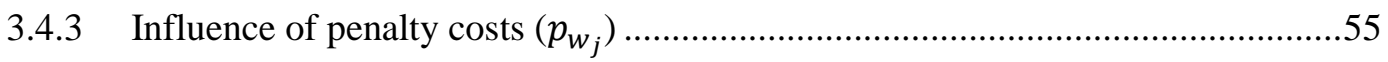

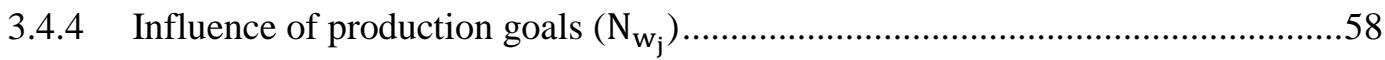

3.4.5 Influence of probabilities that preventive maintenance actions restored the perfect

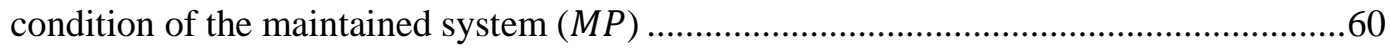

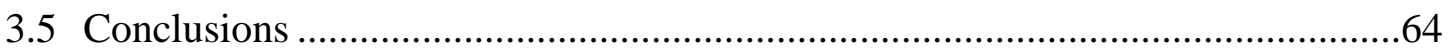

Chapter 4 .................................................................................................................... 65

\section{Integrated Maintenance and Operations Decision Making in Flexible} Manufacturing Systems.................................................................................. 65

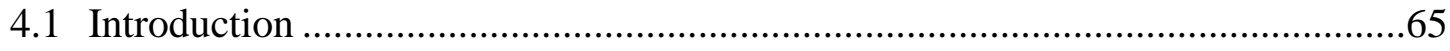

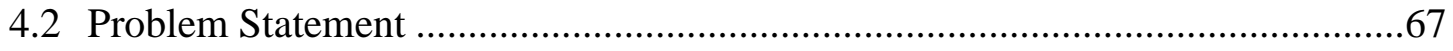

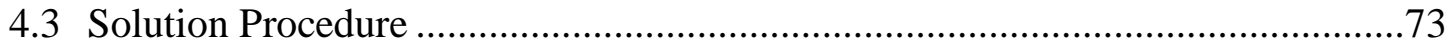

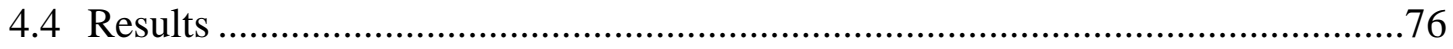

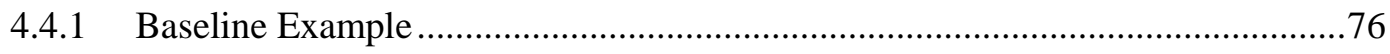

4.4.2 Influence of cheap/regular maintenance cost ratio ..............................................82

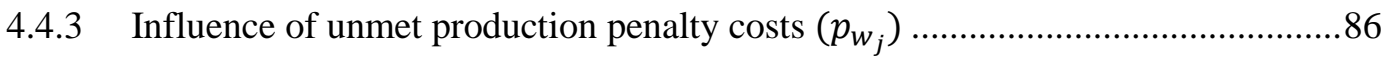

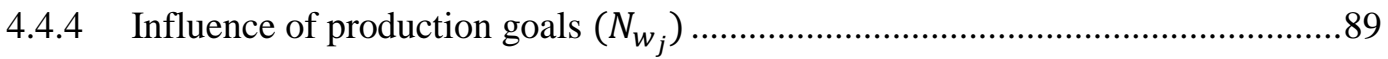

4.4.5 Influence of probabilities that preventive maintenance actions restore the perfect

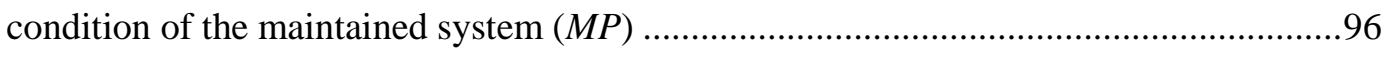

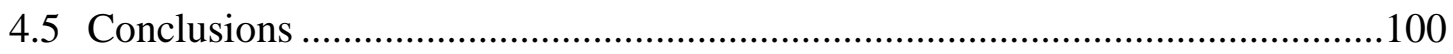


Chapter 5

\section{Integrated Maintenance and Operations Decision Making with} Imperfect Degradation State Observations.................................................... 102

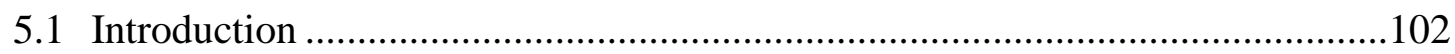

5.2 Overview of POMDP in Integrated Decision-Making ......................................104

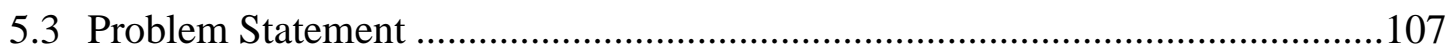

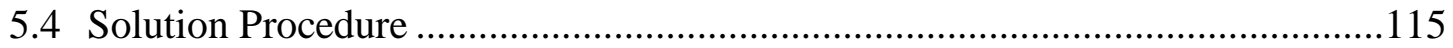

5.4.1 Degradation State Estimation ........................................................................115

5.4.2 Solution Representation ..............................................................................116

5.4.3 Tabu Search Algorithm .................................................................................117

5.4.4 Solution Procedure for Benchmark Policies........................................................119

5.4.4.1 Solution Procedure for Operation-Dependent CBM Policy........................119

5.4.4.2 Solution Procedure for Operation-Independent CBM Policy .....................120

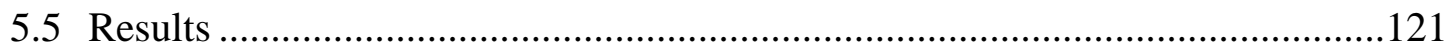

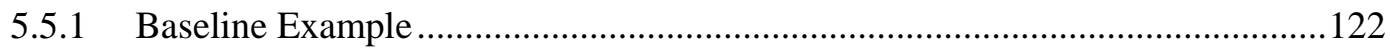

5.5.2 Influence of maintenance probability threshold $(\alpha)$..........................................129

5.5.3 Influence of emission probability matrices $(\mathrm{Q})$.................................................132

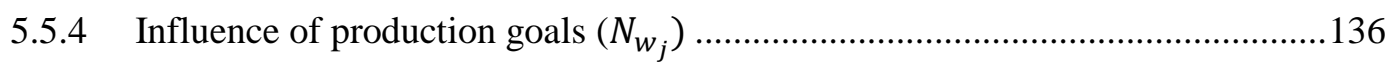

5.5.5 Influence of probabilities that preventive maintenance actions restore the perfect condition of the maintained system $(M P)$.................................................................139

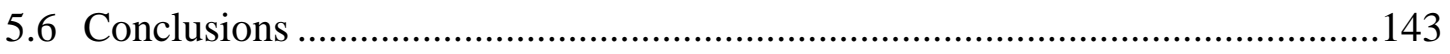

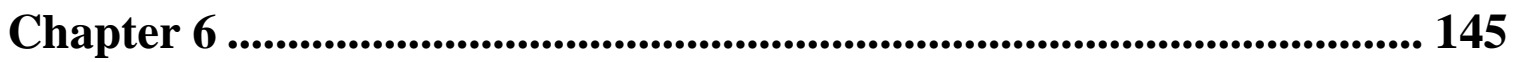

Summary and Future Work ............................................................. 145

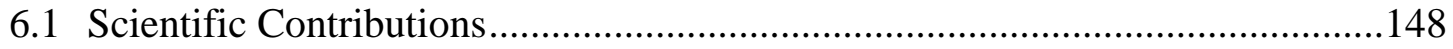

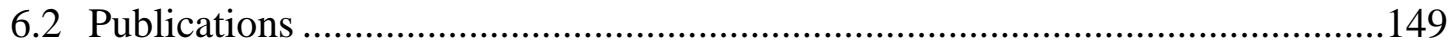

Chapter 7 ...................................................................................................... 151

Literature Review of Methods Relevant to Populating Models ............ 151

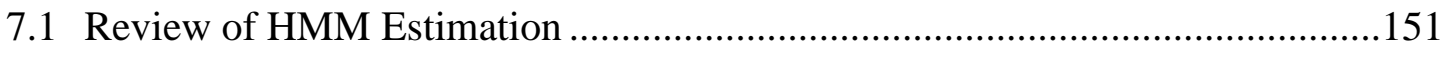

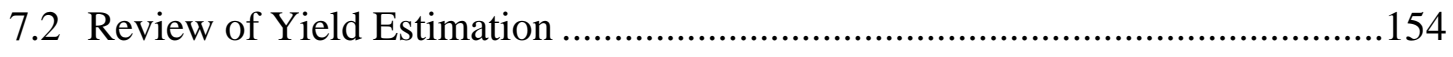




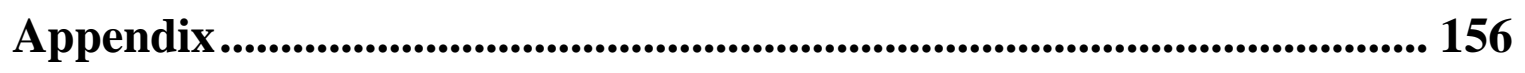
Bibliography ….............................................................................................................. 161 


\section{List of Tables}

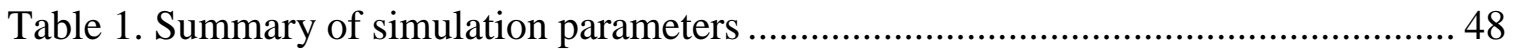

Table 2. Probability of success ("yield") for each operation ..........................................50

Table 3. Parameters of the Tabu Search ...................................................................... 51

Table 4. Summary of simulation parameters for integrated decision-making .................. 79

Table 5. Probability of success for each operation for integrated decision-making ......... 80

Table 6. Parameters of tabu search for integrated decision-making............................... 81

Table 7. p-values for different cheap/regular maintenance cost ratios ............................ 84

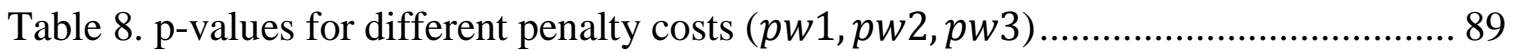

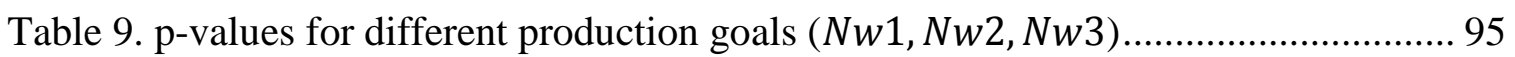

Table 10. $\mathrm{p}$-values for different production goals $(N w 1, N w 2, N w 3)$ with higher penalty

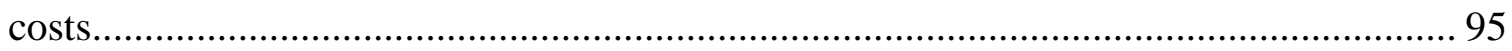

Table 11. p-values for different probabilities of perfect maintenance ........................... 100

Table 12. Summary of simulation parameters for integrated decision-making with imperfect state observations .............................................................................. 124

Table 13. Probability of success ("yield") for each operation for integrated decisionmaking with imperfect state observations........................................................... 125

Table 14. Parameters of Tabu Search for integrated decision-making with imperfect state

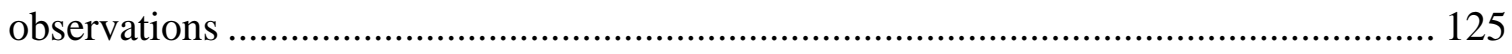

Table 15. p-values for different alpha $(\alpha)$ threshold values ...................................... 131

Table 16. $\mathrm{p}$-values for the same decision-making policy over varying alpha $(\alpha)$ threshold

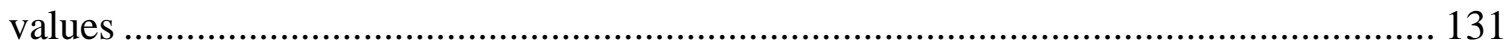

Table 17. p-values for different emission probability matrices ..................................... 134

Table 18. p-values for different production goals $(N w 1, N w 2, N w 3)$ for a system with imperfect state observations.................................................................................... 139

Table 19. p-values for different probabilities of perfect maintenance for a system with imperfect state observations............................................................................... 141 


\section{List of Figures}

Figure 1. Effect of throughput setting change on machine degradation [6] ..................... 3

Figure 2. Solution representation for operation-dependent maintenance decision-making

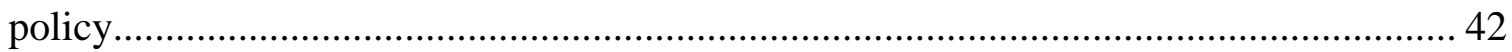

Figure 3. Decision-making by Tabu Search algorithm based on simulations ................. 43

Figure 4. Flowchart of Tabu Search algorithm for operation-dependent maintenance

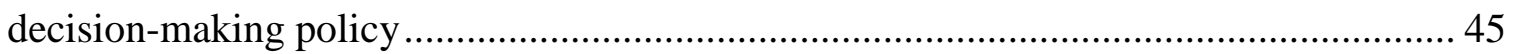

Figure 5. Neighborhood generation for maintenance triggering states........................... 46

Figure 6. Operation-dependent transition probability matrices and maintenance transition

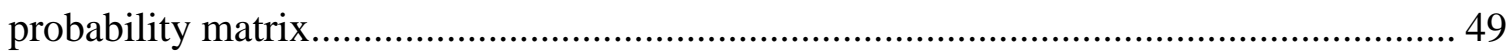

Figure 7. Expected profit comparison for baseline example (black lines denote the $-/+2 \sigma$

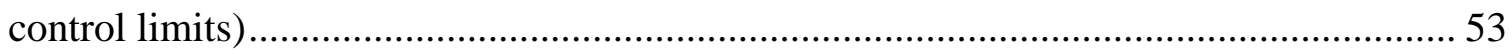

Figure 8. Comparison of expected profits for different reactive maintenance costs (black

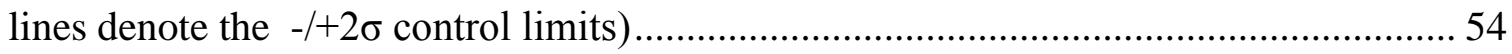
Figure 9. Percent improvement obtained by the operation-dependent maintenance decision-making policy over the operation-independent CBM policy for different reactive maintenance costs 55

Figure 10. Comparison of expected profits for changing penalty costs (black lines denote

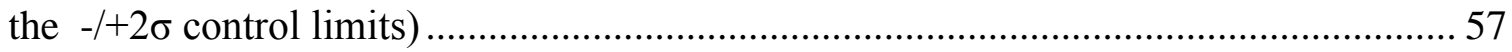

Figure 11. Percent improvement obtained by operation-dependent maintenance decisionmaking policy over the operation-independent CBM policy for different penalty costs . 57 Figure 12. Comparison of expected profits for different production goals (black lines denote the $-/+2 \sigma$ control limits)

Figure 13. Percent improvement over the operation-independent CBM policy obtained by the operation-dependent maintenance decision-making policy for different production goals

Figure 14. Perfect maintenance probabilities used for sensitivity analysis 61

Figure 15. Comparison of expected profits for different perfect maintenance probabilities (black lines denote the $-/+2 \sigma$ control limits).

Figure 16. Percent improvement obtained by the operation-dependent maintenance decision-making policy over the operation-independent CBM policy for different perfect maintenance policies 63

Figure 17. Neighborhood generation for product type sequence. 75 
Figure 18.Operation-specific transition probability matrices and maintenance transition probability matrix for integrated decision making.

Figure 19. Expected profit comparison for integrated decision-making policy

Figure 20. Comparison between the expected numbers of maintenance events conducted during the expensive and cheap maintenance periods 82 Figure 21. Comparison of expected profits for different cheap/regular maintenance cost ratios (black lines denote the $-/+2 \sigma$ limits of the simulation outcomes) for the integrated decision-making policy, operation-dependent $\mathrm{CBM}$ policy and the traditional operationindependent CBM policy..... 84

Figure 22. Comparison of number of regular and cheap PM events for various cost ratios

Figure 23. Percent improvement obtained by the integrated decision-making policy over the benchmark policies for different cheap/regular maintenance cost ratios. 85

Figure 24. Comparison of expected profits for changing unmet production penalty costs (black lines denote the $-/+2 \sigma$ limits of the simulation outcomes) for the integrated decision-making policy, operation-dependent CBM policy and the traditional operationindependent CBM policy....

Figure 25. Percent improvement obtained by the integrated decision-making policy over the benchmark policies for different unmet production penalty costs

Figure 26. Expected total number of PM events for changing penalty costs (black lines denote $-/+2 \sigma$ limits of the simulation outcomes) for the integrated decision-making policy, operation-dependent CBM policy and the traditional operation-independent CBM policy...... 88

Figure 27. Comparison of expected profits for different production goals (black lines denote the $-/+2 \sigma$ limits of the simulation outcomes) for the integrated decision-making policy, operation-dependent CBM policy and the traditional operation-independent CBM policy.

Figure 28. Percent improvement over benchmark policies obtained by the integrated decision-making policy for different production goals.....

Figure 29. Total expected maintenance cost obtained by different maintenance policies for increasing production goals

Figure 30. Probabilities of meeting the production goal for 3 decision-making policies over increasing production goals

Figure 31. Percent improvement over the benchmark policies obtained by the integrated decision-making policy for higher unmet production penalty costs 94 
Figure 32. Perfect maintenance probabilities used for sensitivity analysis with integrated decision-making policy.....

Figure 33. Comparison of expected profits for different probabilities of perfect maintenance (black lines denote the $-/+2 \sigma$ limits of the simulation outcomes) for the integrated decision-making policy, operation-dependent CBM policy and the traditional operation-independent CBM policy....

Figure 34. Percent improvement obtained by the integrated decision-making policy over the benchmark policies for different probabilities of perfect maintenance 99 Figure 35. Representation of a candidate solution for the integrated decision-making policy......

Figure 36. Representation of a candidate solution for the operation-independent CBM policy......

Figure 37. Operation-specific transition probability matrices, maintenance transition probability matrix and emission probability matrix for integrated decision making with imperfect state observations....

Figure 38. Comparison of expected profits for the integrated decision-making policy, operation-dependent CBM policy and the traditional operation-independent CBM policy with imperfect state observations.

Figure 39. Comparison between the expected numbers of maintenance events conducted during the expensive and cheap maintenance periods for a system with imperfect state observations 128

Figure 40. Comparison of expected profits for different alpha $(\alpha)$ values (black lines denote the $-/+2 \sigma$ limits of the simulation outcomes) for the integrated decision-making policy, operation-dependent CBM policy and the traditional operation-independent CBM policy.

Figure 41. Percent improvement obtained by the integrated decision-making policy over the benchmark policies for different alpha $(\alpha)$ values

Figure 42. Emission probability matrices used for sensitivity analysis.

Figure 43. Comparison of expected profits for different emission probability matrices (black lines denote the $-/+2 \sigma$ limits of the simulation outcomes) for the integrated decision-making policy, operation-dependent $\mathrm{CBM}$ policy and the traditional operationindependent CBM policy.....

Figure 44. Percent improvement obtained by the integrated decision-making policy over the benchmark policies for different emission probability matrices. 
Figure 45. Comparison of expected profits obtained by the integrated decision-making policy with imperfect observations using emission probability matrix $Q 5$ and with perfect observations (black lines denote the $-/+2 \sigma$ limits of the simulation outcomes)

Figure 46. Comparison of expected profits for different production goals (black lines denote the $-/+2 \sigma$ limits of the simulation outcomes) for the integrated decision-making policy, operation-dependent CBM policy and the traditional operation-independent CBM policy with imperfect state observations. 138

Figure 47. Percent improvement over benchmark policies obtained by the integrated decision-making policy with imperfect state observations for different production goals

Figure 48. Perfect maintenance probabilities used for sensitivity analysis of the integrated decision-making policy with imperfect state observations

Figure 49. Comparison of expected profits for different probabilities of perfect maintenance (black lines denote the $-/+2 \sigma$ limits of the simulation outcomes) for the integrated decision-making policy, operation-dependent CBM policy and the traditional operation-independent CBM policy with imperfect state observations. 142 Figure 50. Percent improvement obtained by the integrated decision-making policy over the benchmark policies for different probabilities of perfect maintenance for a system with imperfect state observations 


\section{Chapter 1}

\section{Introduction}

\subsection{Motivation and Background}

Maintenance is an essential part of manufacturing operations ensuring that adequate production resources are available to achieve desired productivity and quality in a manufacturing system. Adequate implementation of maintenance operations will ensure that the production system runs smoothly, with as few unexpected breakdowns as possible. However, excessive maintenance will stop the production line, causing both losses in production and an increase in expenses due to unnecessary maintenance operations. Therefore, in order to keep the production line operating smoothly with minimal disruptions and to minimize maintenance costs, it is very important to apply maintenance operations efficiently, on the right equipment at the right time.

Generally speaking, there are two types of maintenance operations; reactive maintenance (RM), which occurs when a tool/machine actually fails and preventive maintenance (PM), which is performed on a tool/machine before actual failure occurs. Even though RM is unavoidable, it usually costs much more and requires more maintenance time when compared to PM [1]. For example, in an automotive assembly plant a minute of unscheduled downtime can cost as much as \$20000 [2]. Also, in semiconductor industry, considering that each wafer is worth up to $\$ 100 \mathrm{~K}$, it is extremely 
important to keep the production line on and the production equipment at a very good condition. Hence, proper scheduling of PM is always desired.

PM policies can be roughly characterized as reliability-based maintenance (RBM) policies, where maintenance is performed at certain time or usage intervals, and condition-based maintenance $(\mathrm{CBM})$ policies, where maintenance is performed when the condition of machine requires a repair. RBM considers the long-run average of the system dynamics and hence the decisions made are not particularly tuned to an individual machine. On the contrary, since CBM is more dynamic and the decisions are based on the condition of the system at that time point, the effects of those decisions can be significantly better than those based on RBM policies [3], enabling preventive maintenance of the system only when necessary, thus, saving resources and improving system availability [4].

In Flexible Manufacturing Systems (FMS), maintenance decisions are considerably harder to make, as the machines have the capability of conducting different manufacturing operations and/or producing at various speeds. In such systems, degradation of a machine depends highly on the operations performed on that machine. Thus, selection of operations executed on a machine directly affects PM decisions by changing the degradation dynamics. On the other hand, PM actions interrupt production and change the system reliability and equipment availability, which in turn directly affects decisions as to which operations should be performed on which piece of equipment [5]. 
Such close interaction between production and maintenance operations decision making is illustrated in Figure 1. Figure 1.a shows a possible scenario of occasionally running a machine with a lower throughput setting, which makes sense especially when the machine is severely degraded. This decelerates machine degradation, lengthens the uptime of the machine, but also lowers productivity. Conversely, as seen in Figure 1.b, a higher throughput setting would accelerate machine degradation and hence increases the probability of machine failure.

(a)

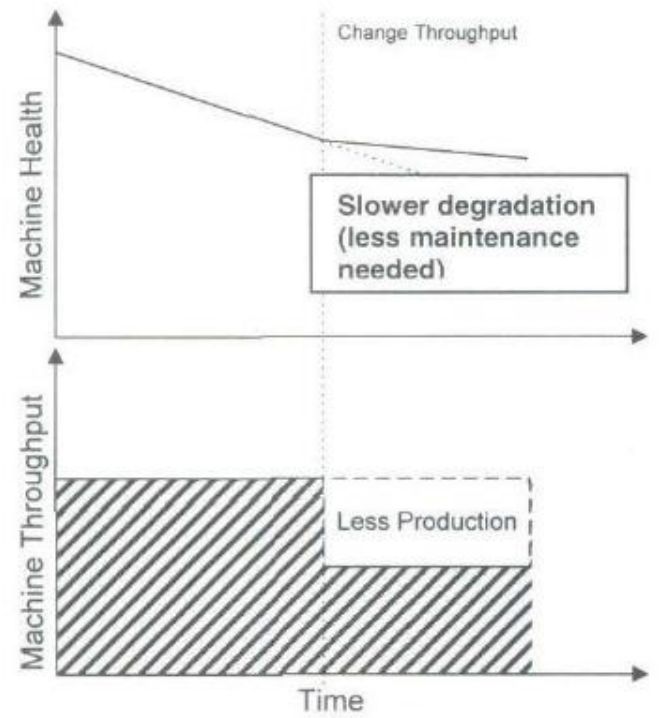

(b)

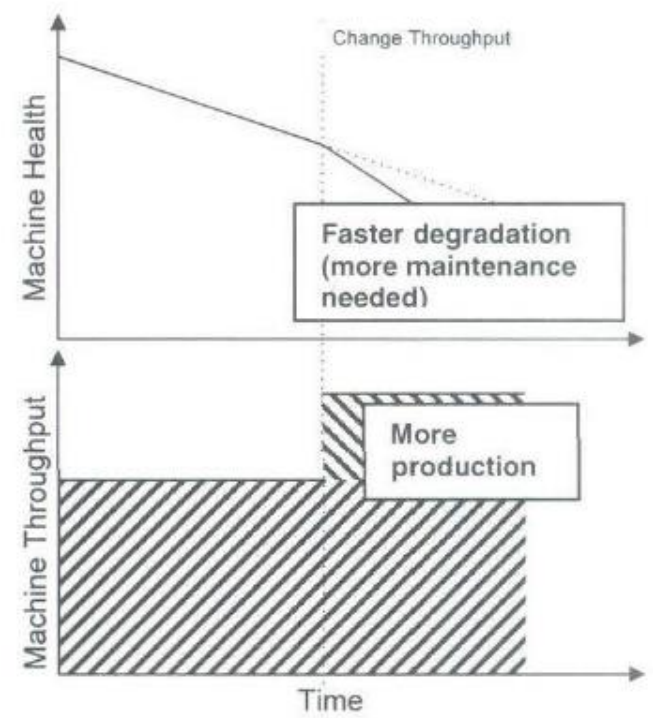

Figure 1. Effect of throughput setting change on machine degradation [6]

In existing literature, most of the maintenance decision-making work is based on modeling the degradation dynamics of single equipment and scheduling maintenance operations for this equipment. However, most production systems, especially flexible manufacturing systems (FMS), are composed of multiple equipment which can execute 
multiple operations. Therefore, there is a need for a decision support tool to schedule the maintenance events and production operations, taking the interactions between equipment and operations executed on these equipment into consideration.

\subsection{Research Objectives and Challenges}

The main objective of the research in this doctoral thesis is to explore various forms of integrated decision making policies for maintenance scheduling and product dispatching in FMS, with the objective of maximizing a customizable profit function with respect to operation-dependent degradation models and production target. Throughout this thesis, the profit function will consist of costs associated with scheduled and unscheduled maintenance, rewards for production and penalties for missed production targets. Simulation based optimization methods are pursued, which will allow one to incorporate various forms of more elaborate cost functions individual users may prefer.

The contributions of this work can be summarized as follows:

1. A maintenance decision making system based on operation-dependent degradation dynamics, where the state of the equipment is perfectly observable.

2. A joint maintenance and operations decision making system where the equipment are prone to operation-dependent degradation and the sequence of the operations executed on the equipment can be arranged in a way to maximize a customizable reward function. 
3. An integrated maintenance and operations decision making system for systems in which the states (condition) of the equipment are not perfectly observable.

The challenges in achieving the aforementioned contributions can be summarized as follows:

1. Even for a small flexible manufacturing system, the state space of possible machine conditions is large, which requires a careful approach to optimization.

2. The sequencing of the products to be produced, and hence the sequencing of the operations to be executed on the equipment, changes the degradation dynamics of the equipment. Therefore, for each operation sequencing option, we would have a different maintenance scheduling policy, which increases the size of the already large solution space.

3. When the states of the equipment are not perfectly observable, the decision making challenges grow further. Namely, the influence of degradation dynamics is now hidden and needs to be inferred from the available sensor readings, which are only probabilistically related to the system condition. This adds another level of complexity to the problem of integrated maintenance and operations decision-making. 


\subsection{Outline of the Dissertation}

The rest of this dissertation is organized as follows. Chapter 2 presents a review of the literature relevant to the proposed research. In Chapter 3, the proposed methodology and the experiments for the operation-dependent maintenance scheduling for flexible manufacturing systems are shown in detail. The work presented in Chapter 3 is based on the paper [7]. Chapter 4 discusses the proposed joint maintenance and operation decision making methodology in detail, along with prior work in this area. Chapter 4 is based on the recently published papers [8] and [9]. Chapter 5 describes the proposed methodology for integrated maintenance and operation decision making where the states of the equipment are not perfectly observable. Finally, conclusions, guidelines for future work and a summary of the scientific contributions of the proposed research are presented in Chapter 6, along with past/foreseen publications to be written based on this doctoral research. 


\section{Chapter 2}

\section{Literature Review}

In order to understand the current state of knowledge on maintenance applications, in Section 2.1 we review the maintenance strategies used in manufacturing. In Section 2.2, optimization methods used in maintenance problems are reviewed. Finally, a review on the existing literature on joint scheduling of maintenance and production operations is given in Section 2.3.

\subsection{Maintenance Strategies in Manufacturing}

All manufacturing systems deteriorate by usage and time. As the deterioration continues, the quality of the products decrease, the costs of operation increases and finally machine breakdown occurs. Maintenance tasks are implemented either to repair the broken down equipment or to prevent breakdowns of the equipment in the first place.

There are mainly two types of maintenance operations; reactive maintenance (RM) which occurs when a tool/machine actually fails and preventive maintenance (PM) which is performed on a tool/machine before actual failure occurs. Preventive maintenance can be grouped as reliability based maintenance (RBM) and condition based maintenance $(\mathrm{CBM})$ as will be explained in detailed in following sections. 
Due to the complexity of the modern manufacturing systems, perfect prediction of failures is impossible and hence it is not possible to completely eliminate unexpected machine breakdowns and reactive maintenance actions. Even though RM is unavoidable, it requires more maintenance time due to unavailability of resources and usually costs three or four times higher when compared to PM ([1], [10]). As mentioned by Iakovou et al. [11], in addition to being less costly, PM prolongs the useful life of the production equipment and keeps the system in an available condition when needed, and the serious loss incurred by the unpredicted fails can be avoided [12]. There are also studies that aim to build up a buffer inventory to use during a maintenance event, which makes PM even more cost effective ([13], [14], [15]).

\subsubsection{Reactive Maintenance}

As aforementioned, due to the complex nature of manufacturing systems, perfect prediction of breakdown events is impossible and hence reactive maintenance operations are unavoidable. However, it is possible to decrease the maintenance time and increase responsiveness through employment of historical data and computers. Kobbacy et al. [16] developed an intelligent maintenance optimization system (IMOS) which uses historical data obtained from past maintenance events. This historical data is used to create a rule based decision support system that gives the maintenance staff guidance concerning which repair model should be applied in a given situation. Through the computer interface and the rule-based decision support system, the maintenance staff is informed 
about a breakdown as soon as it occurs and is also told what kind of maintenance action to take for that specific breakdown event leading to a shortening in downtime duration.

\subsubsection{Preventive Maintenance}

Acknowledging the fact that PM takes less time and is less costly when compared to RM, researchers have focused on finding methods to properly schedule PM and assess its effectiveness. One of the first studies on PM was conducted by Barlow and Hunter [17] in 1960. Summaries on different PM models for various manufacturing systems can be found in the literature surveys of Sherif and Smith [18], Valdez-Flores and Feldman [19], Zeng [20], Fernandez et al. [21] and Garg and Deshmukh [22].

PM policies can be roughly characterized as reliability-based maintenance (RBM) policies, where maintenance is performed at certain time or usage intervals, and condition-based maintenance $(\mathrm{CBM})$ policies, where maintenance is performed when the condition of machine requires a repair. RBM considers the long-run average of the system dynamics and hence the decisions made are not particularly tuned to an individual machine. On the contrary, CBM is more dynamic and the decisions are based on the condition of the system at that time point. Thus, the effects of those decisions can be significantly better than those based on RBM policies [3], which enables preventive maintenance of the system only when necessary, thus, saving resources and improving system availability [4].

Zeng [20] classifies PM into two types as age based maintenance (ABM) and condition based maintenance (CBM). However, ABM models degradation through 
reliability functions and therefore we will review the literature on ABM under reliability based maintenance (RBM).

\subsubsection{Reliability Based Maintenance}

Reliability based maintenance assumes that the breakdown of a machine follows a pattern and the degradation of a machine can be modeled by certain reliability functions. In order to estimate the time between two breakdowns and the time required to restore the system back to its operational state, researchers have used indices such as Mean Time To Failure (MTTF) and Mean Time To Repair (MTTR). Even though MTTF and MTTR are the most commonly used indices, Pham et al. [23] have provided expressions for indices including reliability, availability, life time, operational life time, first failure time, mean life time, mean operational life time and mean time to first failure. Although degradation dynamics of a machine can be different from time to time, these indices would still provide beneficial estimates of breakdown times.

Reliability based maintenance (RBM) is commonly referred to as age-dependent PM or periodic PM in the literature. In age-dependent PM policy, a unit is preventively maintained at predetermined age $T$, or repaired at failure, whichever comes first ([24], [25]). Barlow and Hunter [17] considered age-dependent maintenance policy for both simple and complex systems. For simple systems, such as vacuum tubes, they proposed to maintain the system to "as good as new" state after a certain time of operation. For complex systems, such as computers, they introduced the notion of minimal repair so that the system failure rate is not disturbed after maintenance. Legat et al. [26] presented a 
method for determining optimal age-dependent PM policy for both finite and infinitetime horizons. Canfield [27] and Charles et al. [28] consider a periodic PM policy with perfect maintenance, which means that with each preventive maintenance event the system is restored to as good as new state. Nakagawa [29] discussed optimum PM strategies for a system with both perfect repair and minimal maintenance policies. Another work that considers minimal repair for both preventive maintenance and reactive maintenance can be found in [30]. Aforementioned papers on periodic PM policies have considered either perfect or minimal repair options. However, it is also possible to have an imperfect maintenance option, which causes a variable decrease on the failure rate of the system reliability function. In the work of Chaudhuri and Sahu [31], the reliability of a system with increasing failure rates (IFR) and with imperfect PM was proved to be a concave function and optimum PM intervals for perfect and imperfect PM were found and compared. Other works on periodic PM optimization with imperfect maintenance are presented in [32], [33] and [34]. The work by Linderman et al. [35] demonstrated considerable economic benefits by having an adaptive maintenance policy, where the interval of PM events adapts to the stability of the process.

In aforementioned studies on RBM, systems composed of only a single-unit was considered. However in reality, manufacturing systems are composed of multiple machines which have economic and failure dependencies [1]. An example for economic dependence is the possibility to do PM to non-failed systems at a reduced additional cost, while failed subsystems are being repaired. Failure correlations may be explained by the effects of failure of one subsystem on the other non-failed subsystems. For example, if a 
machine fails, the workload of that machine may be transferred to other non-failed machines, increasing their degradation rates. An example on failure correlations is presented in [36].

Considering these dependencies, Wang [1] classify the RBM policies on multiunit systems as group maintenance policies and opportunistic maintenance policies. Group maintenance policy focuses on the replacement or the repair of a group of machines when one or a certain number of failures occur ([37], [38]). Opportunistic maintenance policy focuses on performing reduced cost PM on non-failed subsystems while the system is stopped due to failed subsystems being repaired. Dagpunar [39] shows that a reduction in maintenance costs can be obtained via opportunistic/interrupt replacements.

\subsubsection{Condition Based Maintenance}

Acknowledging its potential for preventively maintaining the system only when necessary, CBM policies have gained widespread acceptance in recent years. Ahmad and Kamaruddin [40] state that decision making in CBM can be carried out based on two methods: current condition evaluation-based (CCEB) and future condition predictionbased (FCPB). The CCEB method evaluates the current equipment condition, after which the appropriate maintenance is carried out if needed. The FCPB method predicts the future trend of the equipment condition and the appropriate maintenance is planned and scheduled if needed. In this section, we will first review the work based on current 
condition evaluation and then will continue with a review of the recent studies based on predictive CBM.

The work of Christer and Wang [41] is among the first studies to consider condition based maintenance policies. In their work, they addressed the problem of condition monitoring of a component with direct measure information, which they call wear. The decision problem is to choose an appropriate time for the next inspection based upon the condition information obtained to date. If a defective state is identified during an inspection or a failure occurs, the component is immediately repaired or replaced. A similar inspection time decision problem in the context of condition based maintenance is presented in the work of Hontelez et al [42]. Barbera et al. [43] assumed fixed inspection intervals at which the condition of the equipment, such as vibration, is monitored. If the degradation condition of the equipment is above a certain threshold, an instantaneous maintenance action is performed. Aven [44] and Cadini et al. [45] aimed to determine the optimal replacement time of the equipment, which may depend on the information about the condition of the unit. Dieulle at al. [46] not only determined the inspection schedule based on the system state but also the preventive replacement threshold. Similar works on the joint determination of both optimal inspection strategy and optimal repair policy for a manufacturing system whose result is dependent on the system condition can be found in [47], [48] and [49].

Above mentioned studies assumed that inspection to reveal the current condition of the system was made at either regular intervals (the time between two inspections is 
constant) or at irregular intervals (the time between two inspections depends on the current degradation state of the system). There also exist some studies in the literature on CBM that assume continuous monitoring of the system degradation state. For example, Kopnov [50] applied two-level control policies to various types of Markov processes describing degrading parameters of system units. Barata et al. [51] modeled continuously monitored deteriorating systems by using Monte Carlo simulation and embedding the resulting model within an "on condition" maintenance optimization scheme for a multicomponent manufacturing system. Similarly, Marseguerra et al. [4] modeled a continuously monitored multi-component system and used genetic algorithm to determine the optimal degradation level beyond which preventive maintenance has to be performed. Zhou et al. [52] developed a dynamic opportunistic maintenance policy for a continuously monitored multi-unit series system with integrating imperfect effect into maintenance activities. Saassouh et al. [53] proposed an online maintenance decision rule for systems where continuous online information on the actual deterioration levels is available and the systems are subject to a sudden change in their degradation process. Liao et al. [54] determined the optimal reliability threshold $R$ via simulation for a system whose reliability could be monitored continuously and perfectly.

Aforementioned studies on CBM all assumed that the degradation states of the systems were directly observable during inspections. However, it may not be possible for all systems to see the degradation level directly unless there is an obvious failure of the equipment. In such systems, as stated by Wang and Christer [55], the performance of the system concerned cannot be described directly by the monitored information, but is 
correlated with it stochastically. Condition monitoring techniques like vibration and oil analysis can be used to infer the degradation state of the system. Makis and Jiang [56] and Maillart [57] consider such imperfect-information systems where the system states are not observable except failure. The research in [57] models the imperfect-information problem as cost-rate-minimizing partially observable markov decision processes (POMDP).

Njike et al. [58] developed an optimal stochastic control model with objective of minimizing the discounted overall cost due to maintenance activities, inventory holding and backlogs. The deterioration condition of the system is assessed by using the quantity of flawless and defective products and this way they considered the machine failure and human error in an integrated manner.

All aforementioned studies in this section based their decision making on the evaluation of the current condition of the system. Further review of such studies can be found in [40], [59] and [60]. The rest of this section will focus on the review of predictive CBM literature.

Jardine et al. [61] reviews the research and developments in diagnostics and prognostics of mechanical systems implementing CBM with emphasis on models, algorithms and technologies for data processing and maintenance decision-making up to 2005. They state that prognostics, which deals with fault prediction before it occurs, is much more efficient than diagnostics, which deals with fault when it occurs, to achieve zero-downtime performance. 
Yam et al. [62] developed an intelligent predictive decision support system (IPDSS) based on the recurrent neural network (RNN) approach to predict rate of equipment deterioration. The results showed that the IPDSS model provided reliable fault diagnosis and strong predictive power for the trend of equipment deterioration. Wu et al. [63] used artificial neural networks (ANN) to predict the residual life which is updated adaptively online at each sampling instance. This predicted residual life was used to make maintenance decisions. Similarly, in [64], [65] and [66], the maintenance decisions were based on the predicted residual life distributions (RLD) which can be updated using in situ sensor data. The updated distributions are then used to revise the schedule of maintenance events based on the most recently observed degradation information.

In their paper, Garcia et al. [67] presented the application of the Intelligent System for Predictive Maintenance (SIMAP) they developed to the health condition monitoring of a windturbine gearbox. They have shown that SIMAP was able to optimize and dynamically adapt a maintenance calendar for a monitored windturbine according to the real needs and operating life of it as well as other technical and economical criteria.

Lu et al. [68] introduced a predictive CBM model using a state-space model and Kalman filtering to predict future degradation state. These predicted states were then converted to failure probabilities and the maintenance decisions were based on these predicted failure probabilities and associated cost factors.

Both [69] and [70] use predicted system reliability to determine the maintenance schedule that minimizes maintenance cost. Curcurù et al. [69] assume that degradation 
process is observed at regular time intervals, however the observations are imperfect. They show that proposed sensor-driven predictive maintenance policy reduces the maintenance cost with respect to time based maintenance policy. In You et al.'s work [70], the system is monitored continuously and system reliability prediction is updated. It is also assumed in their work that imperfect maintenance is possible and its impacts are captured in the maintenance cost.

Yang et al. [71] focus on the scheduling of the maintenance events by taking the complex interaction between the production process and maintenance operations into account. Effects of any maintenance schedule were evaluated through a discrete-event simulation that utilizes predicted probabilities of machine failures, where predicted probabilities of failure are assumed to be available either from historical equipment reliability information or based on the newly available predictive algorithms ([72]-[74]).

\subsection{Optimization Methods}

Optimization is a method that selects the best element that minimizes or maximizes a certain objective function from a set of elements while taking existing constraints on those elements into consideration. In order to accomplish maintenance optimization, one needs to develop a mathematical model in which both costs and benefits of maintenance are quantified and in which an optimum balance between both is obtained [75]. These models can be of help in determining effective and efficient schedules and plans, that properly consider/incorporate various maintenance policies, 
system configurations, shut-off rules, maintenance restoration degrees, correlation failures and repairs, failure dependence, economic dependence, non-negligible maintenance time, etc [1].

Some researchers have used mathematical models to obtain an analytical solution to the optimization problem. However, for problems that consider a more complex manufacturing system, obtaining analytical solutions is infeasible and hence researchers employed other optimization methods like heuristics, simulation and hierarchical classification for such complex problems.

\subsubsection{Mathematical Methods}

In their paper, Pham et al [23] provided expressions for different indices such as availability, life time, operational life time, first failure time, mean life time, mean operational life time and mean time to first failure. More recently, Chen and Trivedi [76] studied condition-based maintenance and derived closed-form expressions of system availability when the machine undergoes both deterioration as well as Poisson type failures. Those closed-form solutions enabled them to find faster algorithms to determine optimal inspection policy, where they can observe the current degradation state of the system.

Yamayee [77] has claimed that dynamic programming (DP) suits best for maintenance scheduling problems for various reasons. For example, DP is especially suitable for problems where a sequence of decisions is involved, the objective need not be a continuous function of decision and state variables and analytical forms for the 
objective function or constraint functions are not required as long as one can obtain function values. In their study, Zurn and Quintana [78] have presented a state-space representation for the optimal generator maintenance scheduling problem and used successive approximations dynamic programming to find the optimal solution. In order to reduce the size of the state-space, they implement a group-sequential scheduling method instead of a unit-sequential scheduling method. More recently, Barbera et al. [43] obtained the optimal solution via dynamic programming for a condition based maintenance scheduling problem where the objective is to minimize the long-run average cost of maintenance actions and failures.

Maintenance scheduling literature also includes studies that propose using mathematical programming methods for maintenance scheduling problem. For example, Dopazo and Merrill [79] model the generator maintenance scheduling problem as a 0-1 integer programming model and guarantee to find the optimal feasible schedules, if one exists. Egan et al. [80] study the problem of scheduling maintenance activities on generator units in a power system. They model the problem as an integer programming problem and obtain the optimal solution using the branch-and-bound technique. Iakovou et al. [11] uses linear programming to determine the optimal periodic maintenance policy for a system where the rate of deterioration is linked to its throughput. Wang and Pham [32] model the periodic maintenance optimization problem via nonlinear programming for a system with imperfect maintenance actions. 
In some cases, finding the optimal solution for the maintenance planning problem may be infeasible due to long computation times. To address this problem, different numerical approximation methods have been proposed to find near optimal solutions. For example, Tagaras [81] uses the golden section search technique to simultaneously optimize the design parameters for both process control and maintenance procedures. A well-known gradient search algorithm, steepest ascent method was used by Das and Sarkar [82] to find the optimal preventive maintenance policy.

\subsubsection{Metaheuristic Approaches}

The methods based on tractable mathematical models can give a strict optimal solution for small scale problems, but are not efficient for large scale problems because of tremendous number of intermediate solutions (curse of dimensionality). Hence, it is hard to find the optimal solution for large scale problems within a reasonable computing time [83]. Different metaheuristic approaches have been proposed in order to find optimal or near optimal solutions for large scale maintenance planning problems.

The genetic algorithm (GA) is a search heuristic that mimics the process of natural evolution. GA replicates the selection and inheritance principles of the population genetics by implementing "the survival of the fittest" method ([84], [85]). In GA the evolution starts with a randomly generated or, if possible, judiciously picked set of solutions which are represented through numerical strings (chromosomes). In each generation, multiple solutions are stochastically selected from the population based on their fitness values (the more fit the solution, the better chance it has to be selected). 
Through crossover of chromosome pieces and their mutation, a new generation is obtained to be used in the next iteration of the algorithm. As the evolution progresses, the overall fitness of each subsequent generation improves according to the rules of natural selection.

GA has been widely used by researchers to solve the maintenance planning problem. Munoz et al. [86] use the GA at the global and constrained optimization of surveillance and maintenance of components based on risk and cost criteria. Contrary to the common application, they use decimal representation in their chromosomes instead of a binary representation. Cavory et al. [87] encoded the maintenance start times in the chromosomes and evaluated the fitness of each chromosome via a simulation in order to find the solution that yields the highest throughput. Tsai et al. [12] aim to find the activities-combination that maximizes the unit-cost life of the system. They express each activities-combination solution by a string of variable integers, where each variable represent a component of the multi-component system and the allele number on that variable represents the PM activity of that component (no action, simple preventive maintenance, and preventive replacement). Marseguerra and Zio [88] utilize GA to optimize the components' maintenance periods and number of repair teams. The fitness function, which is a profit function which accounts for the safety and economic performance of the plant, is evaluated by Monte Carlo simulation. In a later study, Marseguerra et al. [4] used GA for determining the optimal degradation level beyond which preventive maintenance has to be performed. The fitness function was defined as a multi-objective function of profit and system availability. Ilgin and Tunali [89] proposed 
a GA for the joint optimization of periodic preventive maintenance and spare provisioning policies of a manufacturing system operating in the automotive sector. A $5 \%$ reduction in total annual cost and increase in throughput was obtained by their GA based approach. Wang et al. [90] use GA in a similar problem where condition based maintenance is involved instead of periodic preventive maintenance. Another work on the application of GA on joint planning of maintenance and production is presented by Machani and Nourelafath [91]. Yang et al. [92] used GA for finding the optimal maintenance priority assignment. In the work of Camci [93], for a multi-component system employing condition based maintenance the trade-off between maintenance and failure is quantified in risk as the objective function and minimized utilizing GA for the whole system rather than individual components.

Occasionally, GA is combined with other methods to improve search efficiency. For example, Deris et al. [94] developed a hybrid algorithm that uses GA and constraintbased reasoning (CBR) to optimize the ship availability in Royal Malaysian Navy. The ships are considered available if all of their main systems are in full operational readiness and the availability can be improved by effective maintenance planning and scheduling. Such hybrid and modified genetic algorithm usage has also been studied by Goncalves and Almeida [95] and Chung et al [96].

Simulated annealing (SA) is inspired by annealing in metallurgy, a technique involving heating and controlled cooling of a material to increase the size of its crystals and reduce their defects. This notion of slow cooling is implemented in SA as a slow 
decrease in the probability of accepting worse solutions as it explores the solution space. By accepting worse solutions, a more extensive search of the solution space is allowed. The method was first described by Metropolis et al. [97] and has been improved by Kirkpatrick et al. [98], Cerny [99] and Ingber [100]. SA was applied in the problem of scheduling maintenance for a thermal generator by Satoh and Nara [101]. More recently, Saraiva et al. [102] proposed a SA based approach for the generator maintenance problem but with a different objective function than the one presented in [101].

In some cases, due to their inherent deficiencies, GA and SA alone are not satisfactory. Therefore, to improve the performances of GA and SA, hybrid algorithms combining GA and SA have been proposed for maintenance optimization problems ([83], [103]). These hybrid algorithms were shown to perform better over simple GA and SA.

Tabu Search (TS) is a local search technique that enhances the exploration performance by using advanced memory structures of a computer. Once a candidate solution has been determined, it is marked as 'tabu' so that the same solution is not visited by the algorithm over a certain number of iterations. The search starts from an initial solution (randomly seeded or chosen based on some problem specific information), moves iteratively from a solution $x$ to a "non-tabu" solution $x^{\prime}$ in the local neighborhood of $x$ and terminates when some stopping criterion is satisfied ([104], [105]).

Tabu search as an approach for maintenance scheduling has only recently been applied. Sawa et al. [106] developed an automatic scheduling method for maintenance outage task in power systems using TS. After comparing the results obtained via TS with 
the optimal solution obtained by implicit enumeration, El-Amin et al. [107] claimed that TS offers a viable approach for maintenance scheduling. Gopalakrishnan et al. [108] presented a TS based heuristic (PM_TABU) to find the PM schedule that maximizes the total priority of the scheduled tasks subject to resource availability constraints. PM_TABU was shown to reduce average optimality gap for the test problems. Kim et al. [109] proposed a hybrid algorithm of GA, SA and TS for thermal unit maintenance scheduling problem. The hybrid algorithm adopted the acceptance probability of SA to improve the convergence of simple GA, and TS was introduced to find more accurate solutions.

\subsubsection{Simulation Based Optimization}

Simulations have been widely used in various fields because of their ability to faithfully represent complex systems. Monte Carlo methods are especially useful for modeling complex systems like manufacturing systems. In maintenance optimization problem, simulation has been mostly used as a tool to evaluate a certain maintenance schedule. In their studies, Borgonovo et al. [110] and Marquez [111] used Monte Carlo simulations for assessment of alternative scheduling policies that could be implemented dynamically on the shop floor. However, in the case of too many alternative maintenance schedules, the search for an optimal solution would require an excessively large number of Monte Carlo evaluations. In order to overcome this problem, a hybrid approach of Monte Carlo simulation with GA optimization of decisions has been proposed by researchers for maintenance optimization problems. For example, Marseguerra et al. [4] 
consider a continuously monitored multi-component system and use a GA for determining the optimal degradation level beyond which PM has to be performed. They aim at simultaneously optimizing two objective functions: profit and availability. The estimates for these objective functions for each solution generated by GA are obtained via Monte Carlo simulations. Cavory et al. [87] aims to increase the overall through-put of a single product manufacturing production line by optimizing the schedule of the maintenance tasks of all machines. They developed an optimizer using GA and evaluated potential solutions by a simulator of the production line. The main focus of their study was the tuning of the genetic algorithm parameters. Marseguerra and Zio [88] present an approach to the optimal maintenance and repair strategies of an industrial plant, in the face of reliability and economic constraints, based on the coupling of GA and Monte Carlo techniques. GA is utilized to optimize the components' maintenance periods and number of repair teams. The value of the fitness function is computed by the Monte Carlo simulation model of the system, which enabled them to include several practical aspects such as stand-by operation modes, deteriorating repairs, aging, sequences of periodic maintenances, number of repair teams available for different kinds of repair interventions (mechanical, electronic, hydraulic, etc.) and components priority rankings. Bris et al. [112] employ the coupling of GA and simulation to find the optimal PM model that minimizes the maintenance cost for a system consisting of subsystems connected in series. Yang et al. [71] propose a GA based optimization procedure for scheduling of maintenance operations in a manufacturing system using the continuous assessment and prediction of the level of performance degradation of manufacturing equipment. Effects 
of any maintenance schedule are evaluated through a discrete-event simulation that utilizes predicted probabilities of machine failures in the manufacturing system.

\subsubsection{Decision Trees}

In some maintenance problems, it is important for managers to consider some non-quantitative issues such as safety issues. In such cases, the type of maintenance strategy to follow becomes more important than the timing of the maintenance. For this type of maintenance problems, hierarchical methods, like decision trees, have been used to pick the best maintenance strategy that satisfies all the constraints of the system. Bevilacqua et al. [113] developed Analytical Hierarchy Process (AHP) for selecting the best maintenance strategy for an important oil refinery with about 200 plants in total. Considering various parameters, such as safety, machine importance for the process, maintenance costs, failure frequency, downtime length, operating conditions and machine access difficulty, they give the decisions of what kind of maintenance strategy (corrective, preventive, opportunistic, condition-based, predictive) to use. Deshpande and Mopak ([114], [115]) used logic trees to suggest PM categories for various failure modes in the components in a medium scale steel industry.

\subsection{Joint Scheduling of Maintenance and Production Operations}

Despite the fact that there are dynamic interactions between production and maintenance events, not many researchers have focused on the problem of optimizing production and maintenance jointly. Given that maintenance affects available production 
time and elapsed production time affects the probability of machine failure, this interdependency seems to be overlooked in the literature [116]. The literature on joint optimization of maintenance and production operations can be grouped into two based on the scheduling technique used. The first group considers sequential scheduling of production and maintenance operations; a production schedule is determined first and then maintenance operations are scheduled according to the pre-determined production schedule. The second group, acknowledging the interactions between the effects of PM decisions on system reliability and the effects of production on degradation, optimizes production and maintenance operations simultaneously.

\subsubsection{Sequential Scheduling}

Benbouzid et al [117] presented a sequential strategy for a flow shop environment in which the production tasks are scheduled first and then the tasks of maintenance are integrated, taking the scheduling of production as a strong constraint. In their works, Cassady and Kutanoglu ([116], [118]) suggested to order the jobs according to weighted shortest processing time (WSPT) and then identify the optimal PM decisions for this job sequence for large problems. For smaller problems, they solved the integrated preventive maintenance and production scheduling problem by using complete enumeration method. The first step in their solution procedure was to enumerate the set of all feasible job sequences and the second step was to identify the set of optimal PM decisions for each feasible job sequence. They compared the performance of the integrated solution with the solutions obtained from solving the preventive maintenance planning, and job scheduling 
problems independently and noted that it is possible to obtain improvements of as much as $20 \%$.

A sequential approach is also followed by Benbouzid-Sitayeb et al. [119]. In their study, the jobs are scheduled using Ant System algorithm [120] and then the insertion of maintenance tasks is done by using several heuristics, taking the production scheduling as a hard constraint. Berrichi et al. [121] used genetic algorithm to find the optimal allocation and sequencing of the jobs in a parallel machine environment and determined the best PM decisions based on that job schedule.

\subsubsection{Simultaneous Scheduling}

In Flexible Manufacturing Systems (FMS), PM decisions are considerably harder to make, as the machines have the capability of conducting different manufacturing operations. Since degradation of a machine depends highly on the operations performed on it, selection of operations executed on a machine directly affects PM decisions by changing the degradation dynamics. On the other hand, PM actions interrupt production and change the system reliability and equipment availability, which in turn directly affects decisions as to which operations should be performed, on which pieces of equipment [5]. This inherent coupling between decisions in the domains of PM and production operations necessitates simultaneous consideration and optimization of maintenance and production decision in manufacturing systems. As shown in [117], integrated scheduling of maintenance and production operations provide better results than sequential scheduling of maintenance and production operations. 
The need for simultaneous maintenance and operations decision making in flexible manufacturing systems has been addressed only recently. A group of studies has been conducted on systems that produces in lots and consider usage or age based preventive maintenance ([91], [122]-[126]). The objective of this group of studies is to find the optimal economic production quantity (how much to produce if maintenance action is not taken) and preventive maintenance strategy of the system.

Ben Ali et al. [127] proposed a multi-objective genetic algorithm to solve the simultaneous production and maintenance scheduling in the job shop. They considered both age based and usage based preventive maintenance. It was assumed that the assignment of the jobs to the machines and the number and type of the maintenance tasks to be executed on each machine were predetermined. They used GA to find the best sequencing for these predetermined production and maintenance operations on each machine. Jin et al. [128] also aimed to find the best PM and job sequencing using a GA. However, in their work instead of having a PM event at every $T$ time units, they defined intervals in which PM tasks can be applied and determined the optimal PM strategy accordingly.

Yang et al. [6] combined age-based maintenance (ABM) decisions with operations decision making in an environment where there are only two operation modes available: fast and slow. Naturally, a fast operation mode leads to faster degradation but higher production, and vice versa. This effect is modeled by operation specific reliability functions. In their study, possible schedules are searched using a Genetic Search 
Algorithm [129] and the value of each schedule is evaluated by a discrete event simulation. It was shown in their results that jointly optimizing throughput and maintenance operations results in decreased maintenance time and increased profits.

A more complex situation is considered in Zhou et al [5]. They develop an integrated reconfiguration and ABM policy (IRABM) on a single-product parallel-serial system with reconfiguration capabilities. Reconfigurations consisted of transferring flexible operations (i.e. the operations that can be executed by more than one machine) to a different machine than they were initially assigned to. With reconfiguration comes the ability to improve system throughput and reduce the likelihood of a system-wide failure. Nevertheless, there is also an associated cost of operation transfer from a degraded machine to a less degraded one, necessitating a tradeoff between the benefits and drawbacks of operations reconfiguration. It has been shown in their study that IRABM outperforms ABM in terms of lower expected total cost.

The previous two publications ([5], [6]) modeled operation-dependent degradation through operation specific reliability functions, which makes them very suitable for RBM approaches in maintenance decision-making. Iravani and Duenyas [130] utilized Markov models to model the degradation of the machine conditions, with states of the Markov model representing states of the system degradation. PM is triggered when the degradation state reaches a certain threshold level rather than at fixed time/usage points, which makes this model particularly suitable for PM decision-making in the $\mathrm{CBM}$ context. The objective is to minimize the total average inventory and 
maintenance costs by finding the optimal production and maintenance policy. In other words, at any operational state, based on the inventory available, the optimal policy determines whether the machine should produce one more item, stay idle or be maintained. The study of Yao et al. [131] provided an improvement on the work presented in [130] by not just deciding on whether to produce or maintain but if production option is chosen, how much to produce. In [130] and [131], the authors considered systems which produced only a single type of product and hence they did not consider the effects of producing different products on the degradation dynamics of the system.

There have been some studies in the literature, such as the ones presented by Kazaz and Sloan [132] and Cai et al. [133], that tackle the maintenance and production planning problem in a single-stage multiple-product system. In these studies at each decision epoch, the decision of whether to maintain the machine or not is given based on the current degradation state of the machine. If production option is chosen, the decision of which type of product to produce is given.

Kaufman and Lewis [134] study a single server maintenance problem with workload considerations. Similarly, the work by Zhou et al. [135] use simulation-based optimization in a load-sharing system, where different components of the system share an overall load. In such systems, loads allocated to each machine vary, thus affecting degradation dynamics of each machine, since the degradation of a machine depends on the amount of load assigned to that machine. It was shown that Integrated Load 
Allocation and Condition Based Maintenance Policy introduced in that paper results in increased availability of equipment when compared to the traditional CBM.

As machine condition degrades, the outgoing product quality (yield) usually also decreases. One of the earliest works considering the effects of equipment condition on product quality and incorporating it into maintenance scheduling is reported in [136]. A decision making policy that simultaneously determines maintenance and production schedules for a multiple-product single machine system was developed by considering the fact that the machine condition can affect the yield of different product types differently. In a later study, Sloan [137] extends the work from [136] by considering varying production targets and multiple maintenance actions. In addition, Sloan and Shanthikumar [138] consider multiple machines and add job dispatching decisions to the decision-making process developed in [136]. They compare the performances of a set of predetermined dispatching rules (such as FCFS, SPT, selecting the lot with highest yield, etc.) and several specific maintenance policies (such as fixed state, fixed time, etc.).

More recently, Lee et al. [3] present a novel method on joint CBM and dispatching decision-making with yield considerations in a semiconductor manufacturing environment. Unlike [136], Lee et al. [3] do not consider only a set of a priori determined CBM policies. Instead, CBM policies for different wafer types are determined via discrete event simulation and GA based optimization of maintenance triggering states. The authors report that using wafer type-dependent CBM policies results in increased yields. However, [3] overlooks the fact that degradation is an operation-dependent 
process and assumes that each operation affects the degradation of the equipment in the same way.

In this thesis, we address the aforementioned gaps by pursuing several integrated maintenance and production operations decision-making policies for multipleproduct/multiple-station manufacturing systems, where each product requires several operations for completion. In each model we consider, it is assumed that each manufacturing station can execute a certain subset of operations where degradation states follow a known directly or probabilistically observable operating mode-specific stochastic process. The output quality of the products is assumed to decrease as the condition of the machine degrades according to a known product-specific stochastic model. 


\section{Chapter 3}

\section{Operation-Dependent Maintenance Scheduling in Flexible Manufacturing Systems}

\subsection{Introduction ${ }^{1}$}

In flexible manufacturing systems (FMS), the machines have the capability of conducting different manufacturing operations and/or producing at various speeds. In such systems, degradation of a machine depends highly on the operations performed on that machine. For example, a certain operation or higher production speeds may degrade the system faster whereas another operation or lower production speeds may degrade the system slower. Thus, selection of operations executed on a machine directly affects PM decisions by changing the degradation dynamics. On the other hand, the degradation condition of a machine can affect the outcome ("yield") of various operations differently. An acceptable degradation level for operation $o_{1}$ may not be acceptable for another operation $\mathrm{O}_{2}$ and hence a PM action would be required before executing $\mathrm{O}_{2}$ on that machine.

In order to address this problem, in this chapter, we study the problem of maintenance scheduling in a multiple-product/multiple-machine system where each

\footnotetext{
${ }^{1}$ This chapter is based on the following publication: Celen, M., Djurdjanovic, D., 2012, "Operation-Dependent Maintenance Scheduling in Flexible Manufacturing Systems," CIRP Journal of Manufacturing Science and Technology, vol. 5, pp. 296-308.

M. Celen wrote this publication under D. Djurdjanovic's supervision.
} 
product requires several operations for completion. These operations are executed on non-identical machines, each of which can execute a certain subset of operations. Degradation processes of the machines are modeled as operation-dependent Markov models, with the output quality of the products decreasing as the condition of the machine degrades according to a known product-specific stochastic model. Our aim is to facilitate maintenance decision-making in highly flexible manufacturing systems (where one has the ability to do multiple operations on multiple stations), based on the aforementioned operation-dependent degradation models. The decision-making will be done by maximizing a customizable reward function, taking into account rewards of production and the costs of maintenance operations.

The rest of this chapter is organized as follows. In Section 3.2, we formally define the problem of operation-dependent maintenance scheduling. In Section 3.3, we introduce a novel solution procedure for the problem defined in Section 3.2. A numerical experiment based on the simulation of a semiconductor cluster tool is introduced in Section 3.4. The results collected from this experiment are also enclosed in Section 3.4 in order to assess the performance of the newly proposed optimization methodology and compare it with traditional policies. Section 3.5 discusses the conclusions of this work and possible directions for future work. 


\subsection{Problem Statement}

Let us consider a flexible manufacturing system with $m$ manufacturing stations labeled $c_{1}, c_{2}, \ldots, c_{m}$ and assume that in those stations, we are producing a set of product types $W=\left\{w_{1}, w_{2}, \ldots, w_{l}\right\}$. Let $O=\left\{o_{1}, o_{2}, \ldots, o_{K}\right\}$ be the set of all operations that can be executed by the stations of that manufacturing system. Each product type $w_{j}, w_{j} \in W$, is associated with a sequence of operations $O_{w_{j}}=\left[o_{w_{j}}^{1}, o_{w_{j}}^{2}, \ldots, o_{w_{j}}^{p_{j}}\right]$, where $p_{j}$ is the number of operations needed to manufacture that product type. Since any two product types $w_{j_{1}}$ and $w_{j_{2}}$ may have some common operations, the intersections $O_{w_{j_{1}}} \cap O_{w_{j_{2}}}$, $w_{j_{1}}, w_{j_{2}} \in W$ are not necessarily empty sets. It is assumed that each station $c_{i}$ can execute operations $O_{c_{i}}=\left\{o_{c_{i}}^{1}, o_{c_{i}}^{2}, \ldots, o_{c_{i}}^{q_{i}}\right\} \subset O$, where $q_{i}$ is the number of operations that can be executed by that station. Any two stations $c_{i_{1}}$ and $c_{i_{2}}$ may be able to execute some common operations, which means that $O_{c_{i_{1}}} \cap O_{c_{i_{2}}}$, for $c_{i_{1}}, c_{i_{2}} \in\left\{c_{1}, c_{2}, \ldots, c_{m}\right\}$ are not necessarily empty sets. That means some operations can be executed by more than one station, making it necessary to choose which station to use for a given operation. The goal will be to produce $N_{w_{j}}$ of each product type $w_{j}, w_{j} \in W$, within a certain mission time $T$.

In this study, we acknowledge that different operations have different degradation effects on the stations. We assume that the degradation is perfectly observable and is described via concatenated operation-dependent Markov models. More specifically, the degradation process of each station $c_{i}$ is characterized by a set of operation-dependent 
Markov Chains defined over a common state space $S_{c_{i}}=\left\{1,2, \ldots, M_{c_{i}}\right\}$, with Markov chain states denoting the degradation states of the manufacturing station (1 denoting the "good as new" state and $M_{c_{i}}$ denoting the "failed" state). For any station, the probability of transitioning from state $a$ to state $b$ if operation $o_{k}$ is executed in it is defined as operation-dependent and station dependent $P_{a, b}^{\left(o_{k}\right)}$, which yields operation-dependent state transition matrices $P^{\left(o_{k}\right)}=\left[P_{a, b}^{\left(o_{k}\right)}\right], a \in\left\{1,2, \ldots, M_{c_{i}}\right\}, b \in\left\{1,2, \ldots, M_{c_{i}}\right\}$. All Markov chains are assumed to be unidirectional, modeling the well-accepted intuition that the condition of a station can only worsen over time, unless a maintenance operation is done.

When a maintenance operation is performed, the condition of a station will get better, but it may not necessarily go to the "good as new" state. For any station the probability of transitioning to a better state as a result of a maintenance operation is modeled by station dependent state transition matrices $M P=\left[P_{d, e}\right], d \in\left\{2, \ldots, M_{c_{i}}\right\}$, $e \in\left\{1,2, \ldots, M_{c_{i}}-1\right\}$. This means that, unlike what we see in a lot of existing research, this study acknowledges that PMs do not necessarily restore the perfect condition of the maintained system (good as new), or just restore the functionality of the system without improving its condition (minimal maintenance, or "as bad as before").

Let $S_{c_{i}}^{o_{k}}$ denote the state of station $c_{i}$ that triggers preventive maintenance when operation $o_{k}$ is executed in it. It means that if the current station's degradation state $X_{i}(t)$ is greater than or equal to $S_{c_{i}}^{o_{k}}$, then operation $o_{k}$ could not be performed on that station, unless a maintenance operation is done on it beforehand. Thus, as time passes and 
stations degrade, each station "looses" more and more operations that can be done in it, unless a maintenance action is invoked on it to restore its condition.

In addition, let us take into account the fact that as the degradation level of a station increases, the probability of success of any operation executed on that station decreases. We assume that for any station the probability of success of a given operation is a known function of the operation $o_{k} \in O$ and the station state $X_{i}$, denoted as $s_{c_{i}}\left(o_{k}, X_{i}\right)$

Our objective is to find a combined policy of maintenance triggering states and dispatching of operations across a system that maximizes a reward function that considers the benefits of production, costs of maintenance and penalties for unmet production goals. In this study, it is assumed that the sequence in which products are manufactured is fixed, though this is another decision lever that can (and should) be considered in the future.

More formally, this problem can be defined as follows. Let $\left[d_{1}, \ldots, d_{F}\right]$ denote the fixed sequence in which the products are processed in the FMS, where $d_{q} \in$ $\left\{w_{1}, w_{2}, \ldots, w_{l}\right\}, q=1,2, \ldots, F$ and $F$ is the total amount of products that are fed into the manufacturing system (this is the sequence in which the products are released into the system). Also, let $N_{w_{j}}$ denote the production goal for product type $w_{j} \in\left\{w_{1}, w_{2}, \ldots, w_{l}\right\}$, and let $n_{w_{j}}$ denote the number of good products of type $w_{j}$ produced during mission time T. The operation-dependent CBM policy will be defined by maintenance triggering states 
$S_{c_{i}}^{o_{k}} \in\left\{1,2, \ldots, M_{c_{i}}\right\}$ that are specific for each operation $o_{k} \in O$ and each manufacturing station $c_{i} \in\left\{c_{1}, c_{2}, \ldots, c_{m}\right\}$, and that maximize the objective function

$$
E\left(\sum_{j=1}^{l} R_{w_{j}} n_{w_{j}}-V_{r} c_{r}-V_{p} c_{p}-\sum_{j=1}^{l} a_{w_{j}}\left(N_{w_{j}}-n_{w_{j}}\right) p_{w_{j}}\right)
$$

where

- $R_{w_{j}}$ is the unit reward for good products produced of type $w_{j}, w_{j} \in W$

- $\quad p_{w_{j}}$ is the unit penalty for penalizing unmet production for product type $w_{j}, w_{j} \in$ $W$

- $c_{r}$ denotes the unit cost of reactive maintenance per reactive maintenance event

- $c_{p}$ is the unit cost of reactive maintenance per reactive maintenance event

- $V_{r}$ is the total number of reactive maintenance events

- $V_{p}$ is the total number of preventive maintenance events

- $a_{w_{j}}=\left\{\begin{array}{c}1, \text { if } n_{w_{j}}<N_{w_{j}} \\ 0, \text { otherwise }\end{array}\right.$ for $\forall w_{j} \in W$

In other words, the operation-dependent CBM policy will be defined by the optimization problem

$$
\begin{aligned}
& \underset{j=1}{\operatorname{argmax}} E\left(\sum_{j=1}^{l} R_{w_{j}} n_{w_{j}}-V_{r} c_{r}-V_{p} c_{p}-\sum_{j=1}^{l} a_{w_{j}}\left(N_{w_{j}}-n_{w_{j}}\right) p_{w_{j}}\right) \\
& {\left[\begin{array}{cccc}
S_{c_{1}}^{o_{1}} & S_{c_{2}}^{o_{1}} & \cdots & S_{c_{m}}^{o_{1}} \\
S_{c_{1}}^{o_{2}} & S_{c_{2}}^{o_{2}} & \cdots & S_{c_{m}}^{o_{2}} \\
\vdots & \vdots & \ddots & \vdots \\
S_{c_{1}}^{o_{K}} & S_{c_{2}}^{o_{K}} & \cdots & S_{c_{m}}^{o_{K}}
\end{array}\right]}
\end{aligned}
$$


The optimization problem (1) aims to find the operation-dependent maintenance triggering states $S_{c_{i}}^{o_{k}}$ that maximize the expected reward function in (1), given a fixed product type sequence $\left[d_{1}, d_{2}, \ldots, d_{F}\right]$. Objective function in (1) incorporates the rewards obtained from the completion of good products, as well as the penalties incurred in case the production goal is not met. The expectation operator is applied because of stochastic effects induced by randomness in the reliability of equipment (states of manufacturing stations degrade according to operation specific and machine specific stochastic processes), condition-dependent yields (for each operation and each machine, one has a probability of successfully completing the operation) and repair times (repair times are not deterministic in our study). All these random effects are modeled via discrete event simulations and objective function results obtained from multiple runs of the simulations are averaged to estimate the expected profits corresponding to any given maintenance triggering policy.

In order to assess the performance of the proposed methodology, we will compare our results with the traditional Operation-Independent CBM Policy ([136], [139]), which assumes that the maintenance triggering states for each station are independent of the operation executed in it (i.e. $S_{c_{i}}^{o_{1}}=S_{c_{i}}^{o_{2}}=\cdots=S_{c_{i}}^{o_{K}}=S_{c_{i}}$ for each station $c_{i}$ ). In both of these maintenance decision-making problems, the product type sequence $\left[d_{1}, d_{2}, \ldots, d_{F}\right]$ is assumed to be a priori given, which is consistent with the traditional, fragmented approach to maintenance planning and production scheduling. For completeness, the Operation-Independent CBM Policy is defined as the solution to the optimization problem 


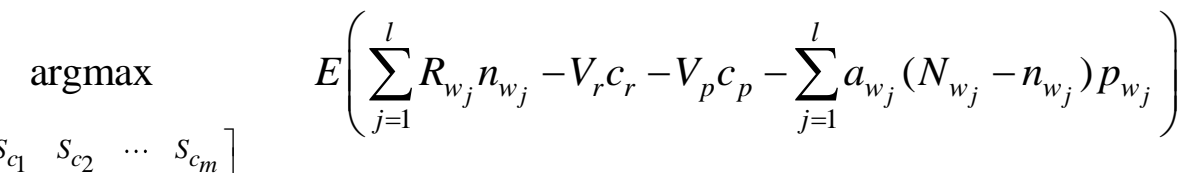

In both maintenance policies, dispatching of the operations to a machine in the cases when an operation can be executed by more than one station is based on the intuitive paradigm of always dispatching it to the least degraded station. This dispatching policy is supported by results from [3]. In addition, both maintenance policies are stationary in the sense that the maintenance triggering states would not change throughout the production of all products.

\subsection{Solution Procedure}

\subsubsection{Operation-Dependent Maintenance Decision-Making}

\subsubsection{Solution Representation}

For an $m$-station manufacturing system with $K$ operations that can be executed in these stations, a solution for preventive maintenance triggering states can be represented with a $K \times m$ matrix illustrated in Figure 2. In this matrix, $i$ th column represents the maintenance triggering states of station $c_{i}$ for each operation, and similarly, $k$ th row represents the triggering states of each station when operation $o_{k}$ is produced in them.

There are up to $\prod_{i=1}^{m}\left(M_{c_{i}}-1\right)^{K}$ candidate solutions in the solution space, though it can be decreased by acknowledging that not all operations can be executed on all stations. Nevertheless, even the aforementioned reduction usually results in a solution 
space that is too large for complete enumeration, especially when candidate solutions are evaluated via replicated discrete-event simulations.

\begin{tabular}{|c|c|c|c|}
\hline$S_{c_{1}}^{o_{1}}$ & $S_{c_{2}}^{o_{1}}$ & $\cdots$ & $S_{c_{m}}^{o_{1}}$ \\
\hline$S_{c_{1}}^{o_{2}}$ & $S_{c_{2}}^{o_{2}}$ & $\cdots$ & $S_{c_{m}}^{o_{2}}$ \\
\hline$\cdots$ & $\cdots$ & $S_{c_{i}}^{o_{k}}$ & $\cdots$ \\
\hline$S_{c_{1}}^{o_{K}}$ & $S_{c_{2}}^{o_{K}}$ & $\cdots$ & $S_{c_{m}}^{o_{K}}$ \\
\hline
\end{tabular}

Candidate solution matrix representation

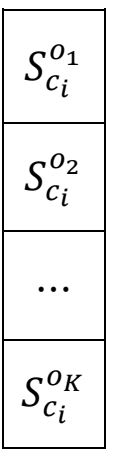

\begin{tabular}{|c|c|c|c|}
\hline$S_{c_{1}}^{o_{k}}$ & $S_{c_{2}}^{o_{k}}$ & $\cdots$ & $S_{c_{m}}^{o_{k}}$ \\
\hline
\end{tabular}

Maintenance policy for operation $o_{k}$

Figure 2. Solution representation for operation-dependent maintenance decision-making policy

Hence, in order to find a practical, applicable and near optimal solution, we used a Tabu Search algorithm [105] based on the results of discrete-event simulations of operations of the underlying manufacturing system. As illustrated in Figure 3, a set of feasible solutions is generated by the Tabu Search algorithm and fed into the discreteevent simulator. The average profit function value relevant to each candidate solution is evaluated from multiple replications of discrete-event simulations of the target manufacturing system and is fed back into the Tabu Search algorithm as the objective function of the optimization process. 


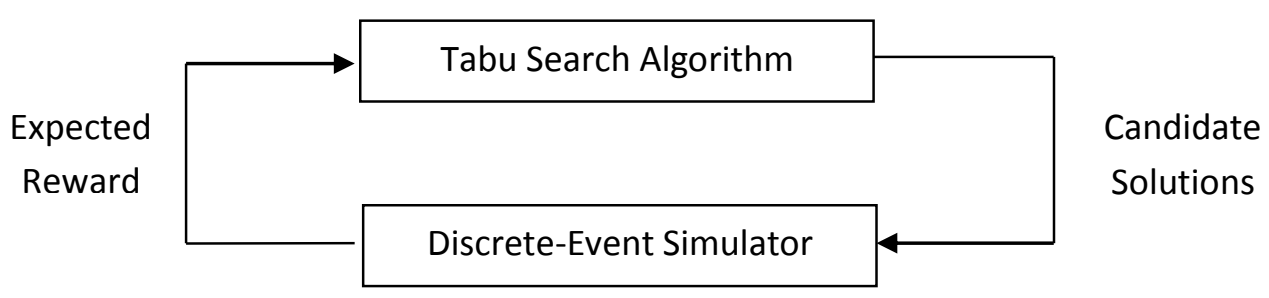

Figure 3. Decision-making by Tabu Search algorithm based on simulations

\subsubsection{Tabu Search Algorithm}

As explained in Section 2.2.3, Tabu Search (TS) has recently begun to be used in maintenance optimization problems. The main idea of TS is to mark a candidate solution as 'tabu' once it has been determined so that the same candidate solution is not visited by the algorithm over a certain number of iterations. The search starts from a randomly seeded or problem specific initial solution and moves iteratively from a non-tabu solution $x$ to another solution $x^{\prime}$ in the local neighborhood of $x$. The flowchart of the Tabu Search algorithm implemented in this study is given in Figure 4.

In our work, to obtain better results in a shorter time, the optimized operationindependent CBM policy was used as the initial solution in the Tabu Search. However, any random solution can be chosen as the initial solution. Elements (candidate solutions) in the local neighborhood of the current solution are generated by randomly selecting a cell in the matrix representing the current solution (shown in Figure 2) and randomly perturbing its value. An example of a local neighborhood for a system with 3 stations, each having 5 degradation states and being capable to execute 4 operations, is illustrated in Figure 5. For this example, the value of each cell can be perturbed to a value in the set 
$\{2, \ldots, 5\}$, since there are 5 degradation states and maintenance is not performed when the machine is at state 1 (the "as good as new" state). In the upper part of Figure 5, the value of the cell at the third row and second column is 4 in the current solution and is perturbed to 2, 3 and 5 to yield 3 elements in the local neighborhood. In the lower part of the figure, the value of the cell in the first row and third column is 5 in the current solution and is perturbed to 2, 3 and 4 to form more elements of the local neighborhood.

A tabu list is implemented to prevent returning to the solutions already visited for a certain number of iterations. However, if the best solution among the candidate solutions is obtained by a tabu move, but yields an expected profit higher than the best profit obtained thus far, then the tabu status of that solution is overridden and it is selected as the next incumbent solution. This is a well documented aspiration criterion concept, often used in Tabu Search [104].

The algorithm terminates whenever the maximum number of iterations is reached or a non-improving move is made for a certain number of consecutive iterations. 


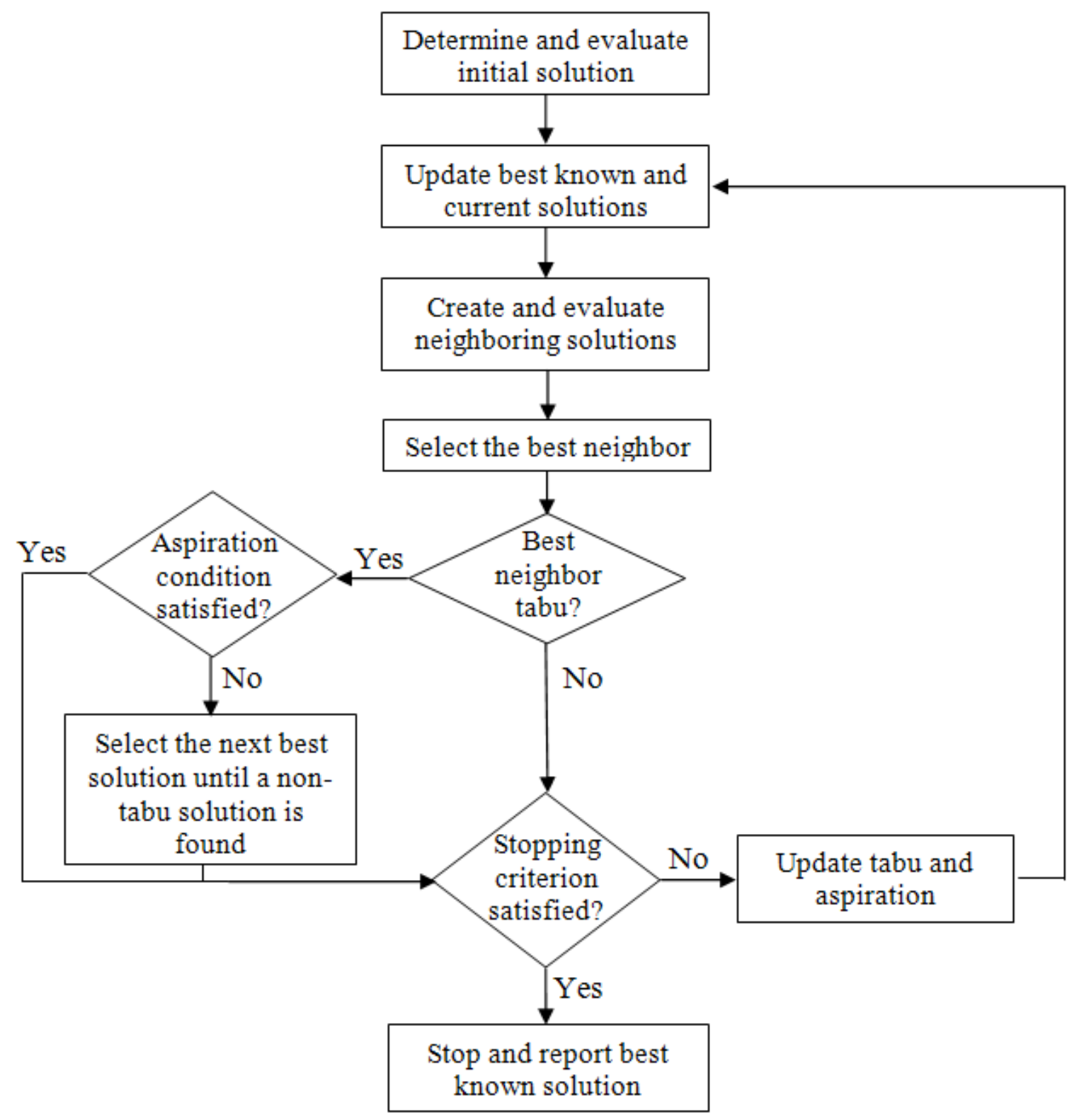

Figure 4. Flowchart of Tabu Search algorithm for operation-dependent maintenance decision-making policy 


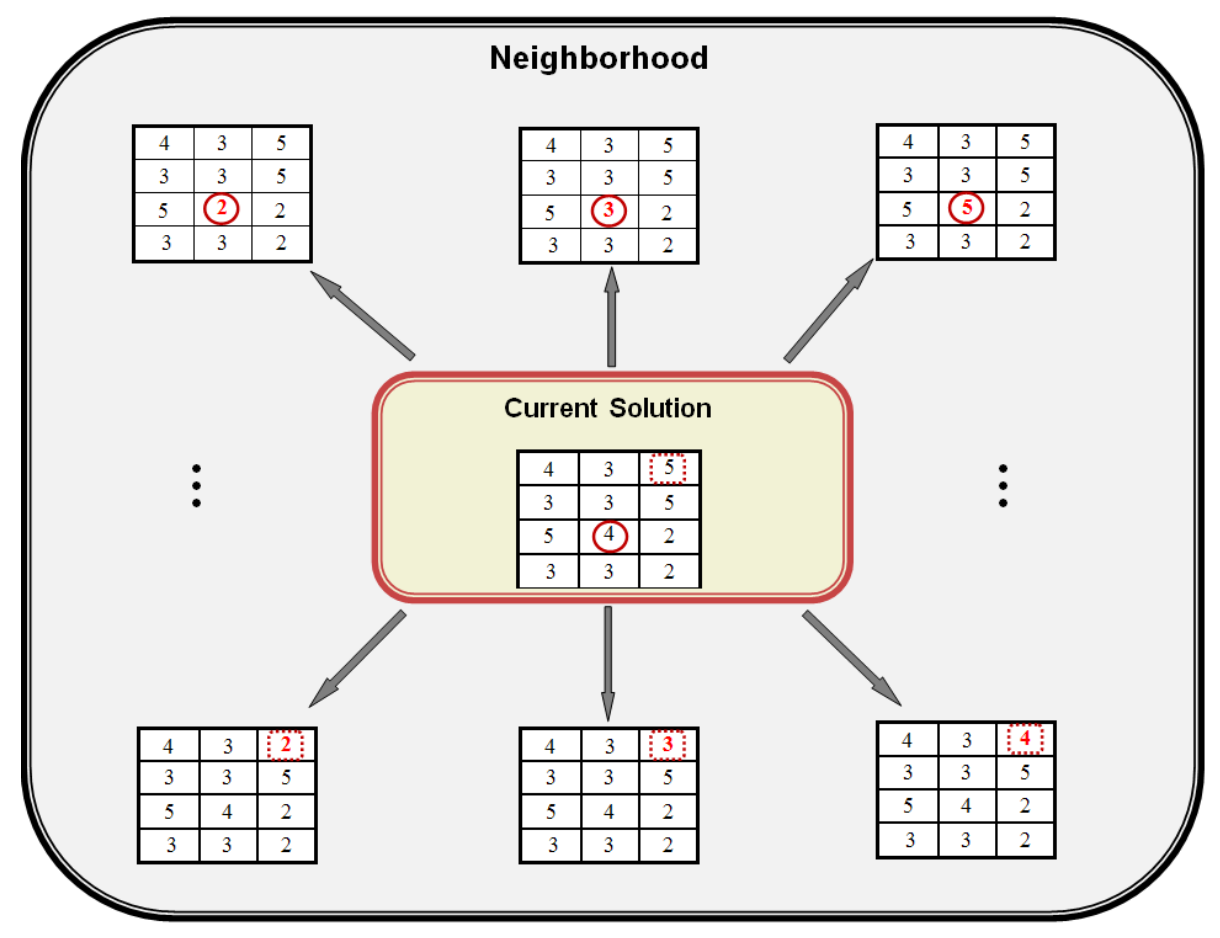

Figure 5. Neighborhood generation for maintenance triggering states

\subsubsection{Operation-Independent CBM Policy Determination}

As mentioned before, the newly proposed operation-dependent maintenance decision-making will be compared with the traditional operation-independent CBM policy obtained by solving the optimization problem (2).

For a manufacturing system with $m$ stations, where station $c_{i}$ has $M_{c_{i}}$ degradation states, the solution space for traditional operation-independent CBM policy optimization consists of up to $\prod_{i=1}^{m}\left(M_{c_{i}}-1\right)$ candidate solutions (since degradation state 1 would never be observed as a potential solution in a CBM policy, as it denotes a trivial policy of maintaining the station after each operation). The solution space for a simple 5-station 
manufacturing system where each station has 5 degradation states consists of $4^{5}=1024$ candidate solutions. Since our focus in this study was on a small manufacturing system (see Section 3.4), this problem can be solved using complete enumeration. The expected profit of each candidate solution is determined by evaluating it via discrete-event simulation of the target system over multiple replications.

\subsection{Results}

Operation-dependent maintenance decision making was tested on an example of a cluster tool, a highly sophisticated and integrated machine routinely used in semiconductor manufacturing. A typical cluster tool is a "mini" manufacturing system of interacting subsystems (multiple chambers as manufacturing stations, supported by a material handling system) and can be seen as a quintessential FMS, since each chamber of a cluster tool can be seen as a manufacturing station and different wafer layers produced in these chambers can be seen as different operations performed in the corresponding manufacturing stations, while different wafer types pushed through the tool can be seen as different product types produced in this system. 
Table 1. Summary of simulation parameters

\begin{tabular}{|c|c|}
\hline Set of chambers & $C=\left\{c_{1}, c_{2}, \ldots, c_{5}\right\}$ \\
\hline Wafer types & $W=\left\{w_{1}, w_{2}, w_{3}\right\}$ \\
\hline $\begin{array}{l}\text { Number of wafers for each } \\
\text { wafer type }\end{array}$ & $\begin{array}{l}F=12, F_{w_{1}}=4, F_{w_{2}}=4, F_{w_{3}}=4 \text { (These are the number of lots and } \\
\text { each lot consists of } 25 \text { wafers, hence } 100 \text { wafers of each wafer type are fed } \\
\text { into the system) }\end{array}$ \\
\hline Wafer type sequence & {$\left[d_{1}, d_{2}, \ldots, d_{12}\right]=\left[w_{1}, w_{2}, w_{3}, w_{1}, w_{2}, w_{3}, w_{1}, w_{2}, w_{3}, w_{1}, w_{2}, w_{3}\right]$} \\
\hline Production goal (wafers) & $N_{w_{1}}=N_{w_{2}}=N_{w_{3}}=67$ \\
\hline Set of operations & $O=\left\{o_{1}, o_{2}, \ldots, o_{13}\right\}$ \\
\hline Operation cycle times ( $\mathrm{min})$ & {$[10,20,15,15,20,20,10,25,10,15,10,10]$} \\
\hline \multirow[t]{2}{*}{$\begin{array}{l}\text { Operation sequences for each } \\
\text { wafer type }\end{array}$} & $o_{w_{1}}=\left[o_{1}, o_{2}, o_{3}, o_{4}, o_{13}, o_{5}\right]$ \\
\hline & $\begin{array}{l}O_{w_{2}}=\left[o_{6}, o_{3}, o_{7}, o_{11}, o_{12}\right] \\
o_{w_{3}}=\left[o_{8}, o_{9}, o_{10}, o_{11}, o_{6}\right]\end{array}$ \\
\hline \multirow[t]{5}{*}{$\begin{array}{l}\text { Operations executable in each } \\
\text { chamber }\end{array}$} & $o_{c_{1}}=\left\{o_{1}, o_{2}, o_{4}, o_{5}, o_{6}, o_{7}, o_{8}, o_{9}\right\}$ \\
\hline & $o_{c_{2}}=\left\{o_{1}, o_{2}, o_{3}, o_{4}, o_{6}, o_{8}, o_{9}, o_{10}\right\}$ \\
\hline & $O_{c_{3}}=\left\{o_{3}, o_{4}, o_{5}, o_{7}, o_{9}, o_{10}, o_{11}\right\}$ \\
\hline & $O_{c_{4}}=\left\{o_{2}, o_{8}, o_{12}, o_{13}\right\}$ \\
\hline & $o_{c_{5}}=\left\{o_{1}, o_{5}, o_{6}, o_{7}, o_{11}, o_{12}\right\}$ \\
\hline Degradation state space & $S_{c_{1}}=S_{c_{2}}=\cdots=S_{c_{5}}=\{1,2, \ldots, 5\}$ \\
\hline \multirow{3}{*}{$\begin{array}{l}\text { Distributions of preventive } \\
\text { maintenance time for each } \\
\text { chamber ( } \mathrm{min})\end{array}$} & {$[U(31,49), U(51,69), U(41,59), U(21,39), U(21,39)]$ and returns to state 1} \\
\hline & {$[U(18,36), U(31,49), U(24,42), U(11,29), U(11,29)]$ and returns to state 2} \\
\hline & {$[U(4,22), U(11,29), U(8,26), U(1,19), U(1,19)]$ and returns to state 3} \\
\hline $\begin{array}{l}\text { Reactive maintenance times for } \\
\text { each chamber ( } \mathrm{min} \text { ) }\end{array}$ & {$[100,110,120,90,100]$} \\
\hline Rewards (\$) & $R_{w_{1}}=50, \quad R_{w_{2}}=25, \quad R_{w_{3}}=10$ \\
\hline Costs (\$) & $c_{p}=50, \quad c_{r}=250, \quad p_{w_{1}}=20, \quad p_{w_{2}}=10, \quad p_{w_{3}}=4$ \\
\hline
\end{tabular}




$$
\begin{aligned}
& P^{\left(o_{1}\right)}=\left[\begin{array}{ccccc}
0.98 & 0.02 & 0 & 0 & 0 \\
0 & 0.98 & 0.02 & 0 & 0 \\
0 & 0 & 0.98 & 0.02 & 0 \\
0 & 0 & 0 & 0.98 & 0.02 \\
0 & 0 & 0 & 0 & 1
\end{array}\right] \quad P^{\left(o_{2}\right)}=\left[\begin{array}{ccccc}
0.90 & 0.08 & 0.02 & 0 & 0 \\
0 & 0.90 & 0.08 & 0.02 & 0 \\
0 & 0 & 0.90 & 0.08 & 0.02 \\
0 & 0 & 0 & 0.93 & 0.07 \\
0 & 0 & 0 & 0 & 1
\end{array}\right] \\
& P^{\left(o_{3}\right)}=\left[\begin{array}{ccccc}
0.93 & 0.05 & 0.02 & 0 & 0 \\
0 & 0.93 & 0.05 & 0.02 & 0 \\
0 & 0 & 0.93 & 0.05 & 0.02 \\
0 & 0 & 0 & 0.97 & 0.03 \\
0 & 0 & 0 & 0 & 1
\end{array}\right] \quad P^{\left(o_{4}\right)}=\left[\begin{array}{ccccc}
0.94 & 0.04 & 0.015 & 0.005 & 0 \\
0 & 0.94 & 0.04 & 0.015 & 0.005 \\
0 & 0 & 0.94 & 0.04 & 0.02 \\
0 & 0 & 0 & 0.94 & 0.06 \\
0 & 0 & 0 & 0 & 1
\end{array}\right] \\
& P^{\left(o_{5}\right)}=\left[\begin{array}{ccccc}
0.98 & 0.015 & 0.005 & 0 & 0 \\
0 & 0.98 & 0.015 & 0.005 & 0 \\
0 & 0 & 0.98 & 0.015 & 0.005 \\
0 & 0 & 0 & 0.98 & 0.02 \\
0 & 0 & 0 & 0 & 1
\end{array}\right] \quad P^{\left(o_{6}\right)}=\left[\begin{array}{ccccc}
0.95 & 0.04 & 0.01 & 0 & 0 \\
0 & 0.95 & 0.04 & 0.01 & 0 \\
0 & 0 & 0.95 & 0.04 & 0.01 \\
0 & 0 & 0 & 0.95 & 0.05 \\
0 & 0 & 0 & 0 & 1
\end{array}\right] \\
& P^{\left(o_{7}\right)}=\left[\begin{array}{ccccc}
0.93 & 0.04 & 0.02 & 0.01 & 0 \\
0 & 0.93 & 0.04 & 0.02 & 0.01 \\
0 & 0 & 0.93 & 0.06 & 0.01 \\
0 & 0 & 0 & 0.97 & 0.03 \\
0 & 0 & 0 & 0 & 1
\end{array}\right] \quad P^{\left(o_{8}\right)}=\left[\begin{array}{ccccc}
0.97 & 0.03 & 0 & 0 & 0 \\
0 & 0.97 & 0.03 & 0 & 0 \\
0 & 0 & 0.97 & 0.03 & 0 \\
0 & 0 & 0 & 0.97 & 0.03 \\
0 & 0 & 0 & 0 & 1
\end{array}\right] \\
& P^{\left(o_{9}\right)}=\left[\begin{array}{ccccc}
0.90 & 0.05 & 0.03 & 0.02 & 0 \\
0 & 0.90 & 0.05 & 0.03 & 0.02 \\
0 & 0 & 0.90 & 0.07 & 0.03 \\
0 & 0 & 0 & 0.90 & 0.10 \\
0 & 0 & 0 & 0 & 1
\end{array}\right] \quad P^{\left(o_{10}\right)}=\left[\begin{array}{ccccc}
0.99 & 0.01 & 0 & 0 & 0 \\
0 & 0.99 & 0.01 & 0 & 0 \\
0 & 0 & 0.99 & 0.01 & 0 \\
0 & 0 & 0 & 0.99 & 0.01 \\
0 & 0 & 0 & 0 & 1
\end{array}\right] \\
& P^{\left(o_{11}\right)}=\left[\begin{array}{ccccc}
0.89 & 0.07 & 0.03 & 0.01 & 0 \\
0 & 0.89 & 0.07 & 0.03 & 0.01 \\
0 & 0 & 0.91 & 0.07 & 0.02 \\
0 & 0 & 0 & 0.91 & 0.09 \\
0 & 0 & 0 & 0 & 1
\end{array}\right] \quad P^{\left(o_{12}\right)}=\left[\begin{array}{ccccc}
0.92 & 0.06 & 0.02 & 0 & 0 \\
0 & 0.92 & 0.06 & 0.02 & 0 \\
0 & 0 & 0.92 & 0.06 & 0.02 \\
0 & 0 & 0 & 0.95 & 0.05 \\
0 & 0 & 0 & 0 & 1
\end{array}\right] \\
& P^{\left(o_{13}\right)}=\left[\begin{array}{ccccc}
0.95 & 0.03 & 0.02 & 0 & 0 \\
0 & 0.95 & 0.03 & 0.02 & 0 \\
0 & 0 & 0.95 & 0.03 & 0.02 \\
0 & 0 & 0 & 0.96 & 0.04 \\
0 & 0 & 0 & 0 & 1
\end{array}\right] \quad M P=\left[\begin{array}{ccccc}
0 & 0 & 0 & 0 & 0 \\
1 & 0 & 0 & 0 & 0 \\
0.60 & 0.40 & 0 & 0 & 0 \\
0.60 & 0.30 & 0.10 & 0 & 0 \\
1 & 0 & 0 & 0 & 0
\end{array}\right]
\end{aligned}
$$

Figure 6. Operation-dependent transition probability matrices and maintenance transition probability matrix 
In order to assess the performance of the newly proposed maintenance decisionmaking methodology, we used the AutoMod software package [140] to simulate a 5chamber cluster tool, producing 3 types of wafers. Throughout our study, we used 40 simulation replications to evaluate each candidate solution for a maintenance policy. We determined this number by running the simulations over a wide spectrum of reliability and cycle time parameters and by increasing the number of replications until further increase of this number did not result in significant changes in the average profits obtained from the simulations. There was no control over the random number seeds that were used in these replications. It also should be noted that, at the end of each simulation, the current condition of the system is not maintained.

Table 2. Probability of success ("yield") for each operation

\begin{tabular}{|l|ccccc|}
\cline { 2 - 6 } \multicolumn{1}{c|}{} & State 1 & State 2 & State 3 & State 4 & State 5 \\
\hline $\boldsymbol{o}_{\mathbf{1}}$ & 1 & 0.9606 & 0.8295 & 0.8122 & 0.7938 \\
\hline $\boldsymbol{o}_{\mathbf{2}}$ & 1 & 0.9900 & 0.9327 & 0.9132 & 0.8896 \\
\hline $\boldsymbol{o}_{\mathbf{3}}$ & 1 & 0.9224 & 0.6022 & 0.5677 & 0.4935 \\
\hline $\boldsymbol{o}_{\mathbf{4}}$ & 1 & 0.9801 & 0.8877 & 0.8619 & 0.8284 \\
\hline $\boldsymbol{o}_{\mathbf{5}}$ & 1 & 0.9512 & 0.8650 & 0.7643 & 0.7438 \\
\hline $\boldsymbol{o}_{\mathbf{6}}$ & 1 & 0.9276 & 0.9110 & 0.8946 & 0.8525 \\
\hline $\boldsymbol{o}_{\mathbf{7}}$ & 1 & 0.9317 & 0.8386 & 0.5721 & 0.5062 \\
\hline $\boldsymbol{o}_{\mathbf{8}}$ & 1 & 0.9631 & 0.8301 & 0.7737 & 0.7316 \\
\hline $\boldsymbol{o}_{\mathbf{9}}$ & 1 & 0.9360 & 0.9042 & 0.7430 & 0.6866 \\
\hline $\boldsymbol{o}_{\mathbf{1 0}}$ & 1 & 0.9511 & 0.9397 & 0.9284 & 0.8991 \\
\hline $\boldsymbol{o}_{\mathbf{1 1}}$ & 1 & 0.9500 & 0.8758 & 0.8418 & 0.7084 \\
\hline $\boldsymbol{o}_{\mathbf{1 2}}$ & 1 & 0.9739 & 0.9605 & 0.8880 & 0.8603 \\
\hline $\boldsymbol{o}_{\mathbf{1 3}}$ & 1 & 0.9276 & 0.7848 & 0.7169 & 0.6354 \\
\hline
\end{tabular}




\subsubsection{Baseline Example}

The parameters used in the baseline example are given in Table 1, Table 2 and Figure 6. Operation-independent CBM policy was determined through complete enumeration and $S_{C B M}=\left[\begin{array}{lllll}3 & 3 & 3 & 3 & 4\end{array}\right]$ was found to be the best operation-independent CBM policy in the baseline parameter setting.

In order to optimize the operation-dependent decision-making policy, Tabu Search algorithm described in Section 3.3.1.2 was used. The parameters of the Tabu Search algorithm used in this study were selected in an ad hoc manner and are given in Table 3. General guidelines for a more systematic Tabu Search parameter selection can be found in [141].

Table 3. Parameters of the Tabu Search

\begin{tabular}{|l|c|}
\hline \multicolumn{1}{|c|}{ Parameters of Tabu Search } & Value of Parameters \\
\hline Maximum iterations until which the algorithm will run & 20 \\
\hline Tabu list length & 5 \\
\hline Neighbors generated for maintenance triggering states & 10 \\
\hline Replication number to find expected profit & 40 \\
\hline
\end{tabular}

One upper bound for both maintenance policies is an ideal scenario where there are no PM events, no RM events and no scraps. Therefore, the total rewards from production with no scrap, i.e. 8500 , is an upper bound for both maintenance policies. We can compare this upper bound to the results obtained by the proposed operationdependent CBM policy and benchmark operation-independent CBM policy. 
Let us first note that, as illustrated in Figure 7, the operation-dependent maintenance decision-making policy results in a higher expected profit, when compared to the traditional operation-independent CBM policy.

Second, let us inspect the maintenance triggering states obtained by the operationdependent maintenance decision-making policy and enclosed in Appendix A.1. For the best operation-dependent maintenance decision-making policy, it can be observed that the maintenance triggering states $S_{c_{2}}^{O_{9}}$ and $S_{c_{3}}^{o_{9}}$ have been changed from 3 (the state implied by the operation-independent CBM policy) to 4 , whereas $S_{c_{2}}^{o_{10}}$ has changed from 3 to 5. Operations $o_{9}$ and $o_{10}$ are both executed only in the manufacturing of the cheapest wafer and this result proves the intuition that as the wafer reward decreases, the effect of completing that wafer successfully on the profit decreases, thus favoring later triggering of maintenance. In addition to that, the maintenance triggering states $S_{c_{2}}^{o_{3}}$ and $S_{c_{3}}^{o_{4}}$ have changed from 3 to 2 , whereas $S_{c_{5}}^{o_{12}}$ has changed from 4 to 3 . Operations $o_{3}, o_{4}$ and $o_{12}$ are executed in the manufacturing of more costly wafers and degrade the chambers faster. Therefore, this result proves the intuition that more frequent maintenance (corresponding to earlier triggering of maintenance) is optimal for faster degrading operations, as well as the intuition that increased wafer rewards favor earlier triggering of maintenance. 


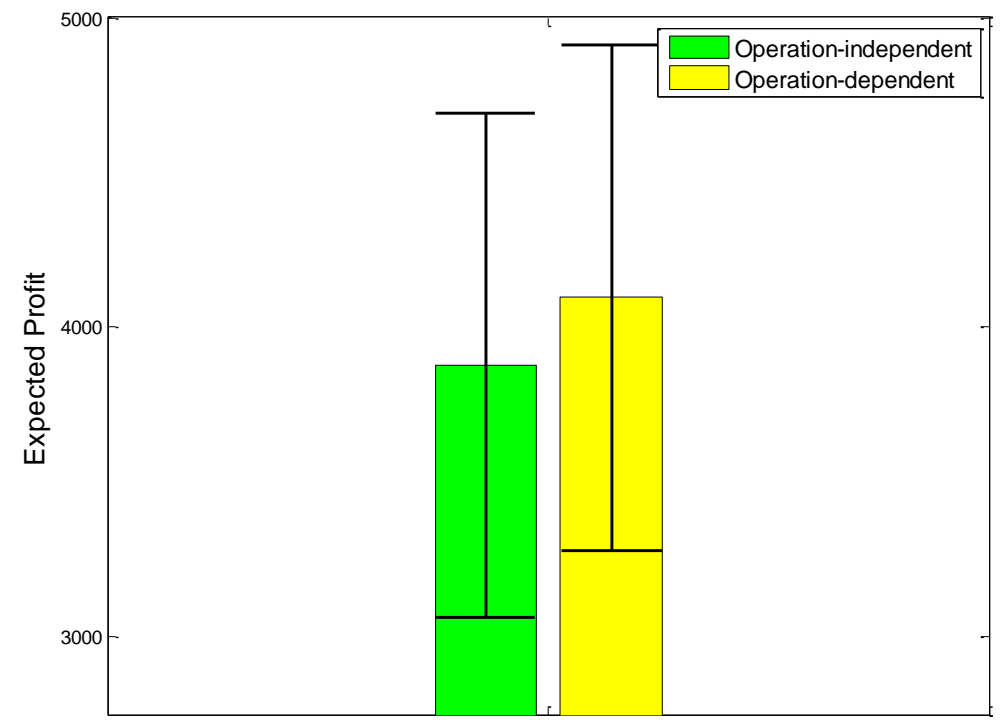

Figure 7. Expected profit comparison for baseline example (black lines denote the $-/+2 \sigma$ control limits)

To gain better understanding of the operation-dependent maintenance decisionmaking policy, we studied the influences of reactive maintenance cost $\left(c_{r}\right)$, penalty cost $\left(p_{w_{j}}\right)$ and production goal $\left(N_{w_{j}}\right)$ parameters, as well as the efficacy of maintenance execution on the resulting decisions.

\subsubsection{Influence of reactive maintenance $\operatorname{cost}\left(c_{r}\right)$}

The expected profits obtained for various reactive maintenance costs $c_{r}$ are shown in Figure 8, while the percent improvements obtained with operation-dependent maintenance decision-making policy over the operation-independent CBM policy are given in Figure 9 (see Appendix A.2 for numerical values of the maintenance triggering 
state results). As observed in Figure 8, for all reactive maintenance costs, operationdependent CBM policy results in a higher expected profit when compared to the benchmark policy (operation-independent CBM). Furthermore, as illustrated in Figure 9, the benefits of the operation-dependent CBM policy increase as the reactive maintenance costs increase. This is intuitively plausible since the operation-dependent maintenance decision-making policy decreases the probability of unexpected failures and consequent reactive maintenance events. The reason for this is that it finds the most appropriate maintenance triggering states for each operation, rather than just one maintenance triggering state for all operations, which is what traditional operation-independent CBM policy does.

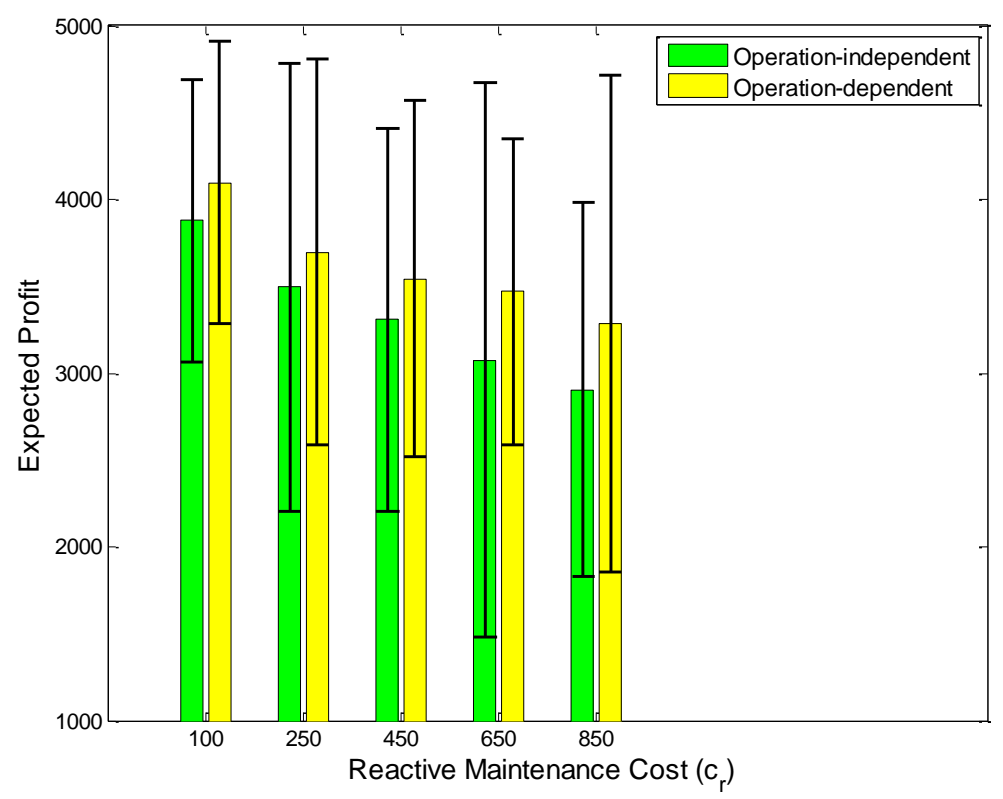

Figure 8. Comparison of expected profits for different reactive maintenance costs (black lines denote the $-/+2 \sigma$ control limits) 


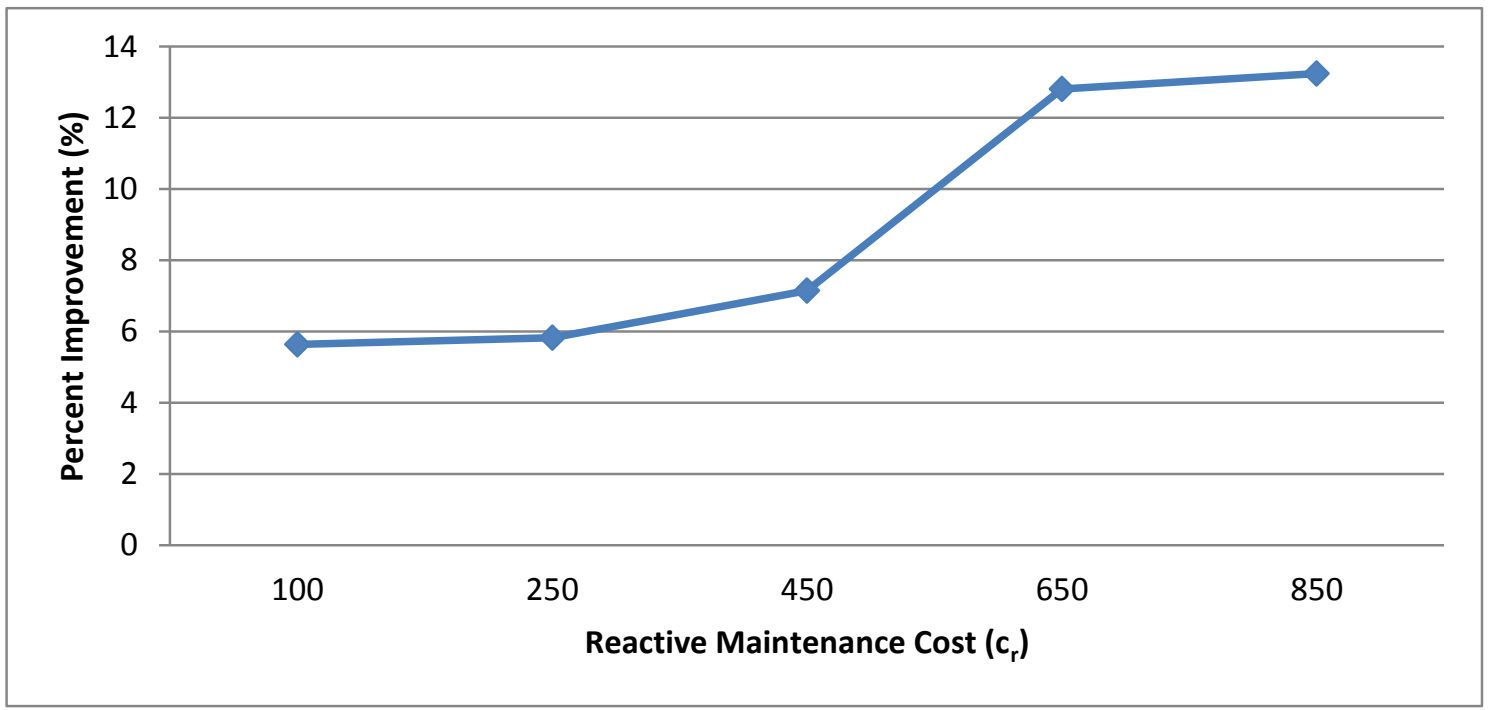

Figure 9. Percent improvement obtained by the operation-dependent maintenance decision-making policy over the operation-independent CBM policy for different reactive maintenance costs

\subsubsection{Influence of penalty costs $\left(\mathbf{p}_{w_{j}}\right)$}

In this section, we investigate the performance of the newly proposed operationdependent maintenance decision-making policy under different penalty cost configurations. The expected profit and percent improvement results for increasing penalty costs are given in Figure 10 and Figure 11 (for numerical values of the corresponding results, please see Appendix A.3). Once again, it is visible from Figure 10 that the expected profits obtained with the operation-dependent CBM policy are higher than the profits obtained by the operation-independent CBM policy, regardless of the penalty cost parameters. Also, a closer look at the figure shows that as the penalty costs for the wafers change, the expected profits obtained with the operation-dependent CBM policy vary slightly. However, the variance of the expected profits for the operation- 
independent CBM policy is higher than the operation-dependent CBM policy. A simple explanation for this situation is that even under different penalty costs, operationdependent maintenance decision-making policy manages to meet the production goal more often and hence is not significantly affected by the changes in the penalty costs. On the other hand, the operation-independent CBM policy is less likely to meet the production goal and hence results in reduced profits and more uncertainty in the profits as the penalty costs increase.

This reasoning also explains the improvement pattern observed in Figure 11. The operation-dependent maintenance decision-making policy pursues maintenance triggering states that maximize the probability of successful completion of an operation and thus minimizes the number of scrap products. Therefore, it is intuitive that the relative benefits of the operation-dependent maintenance decision-making policy over the operationindependent CBM policy become larger as the penalty costs increase. 


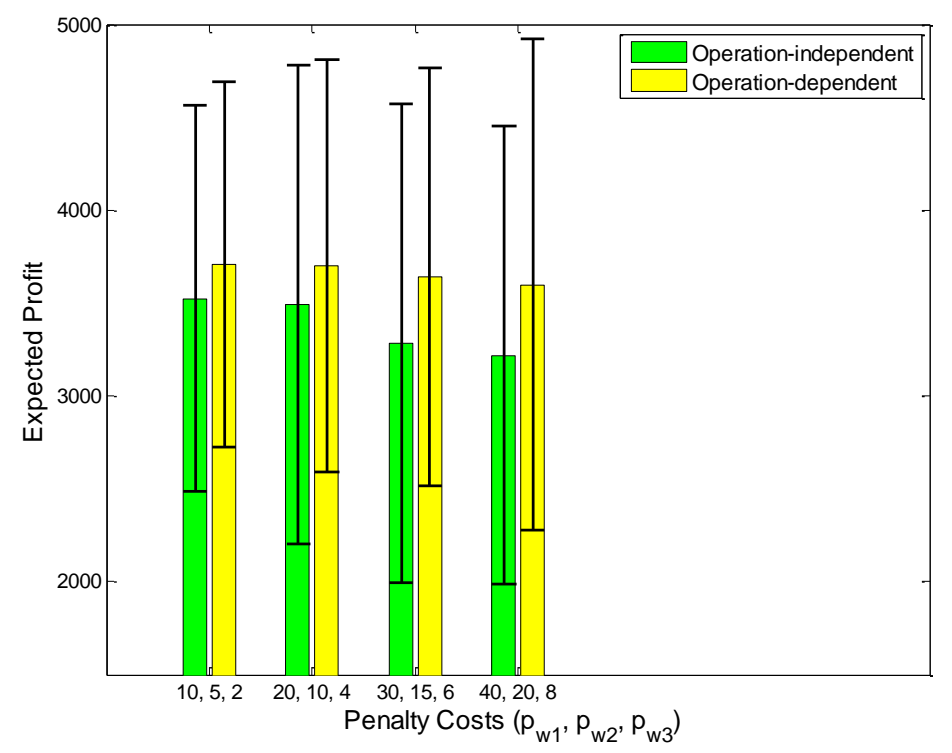

Figure 10. Comparison of expected profits for changing penalty costs (black lines denote the $-/+2 \sigma$ control limits)

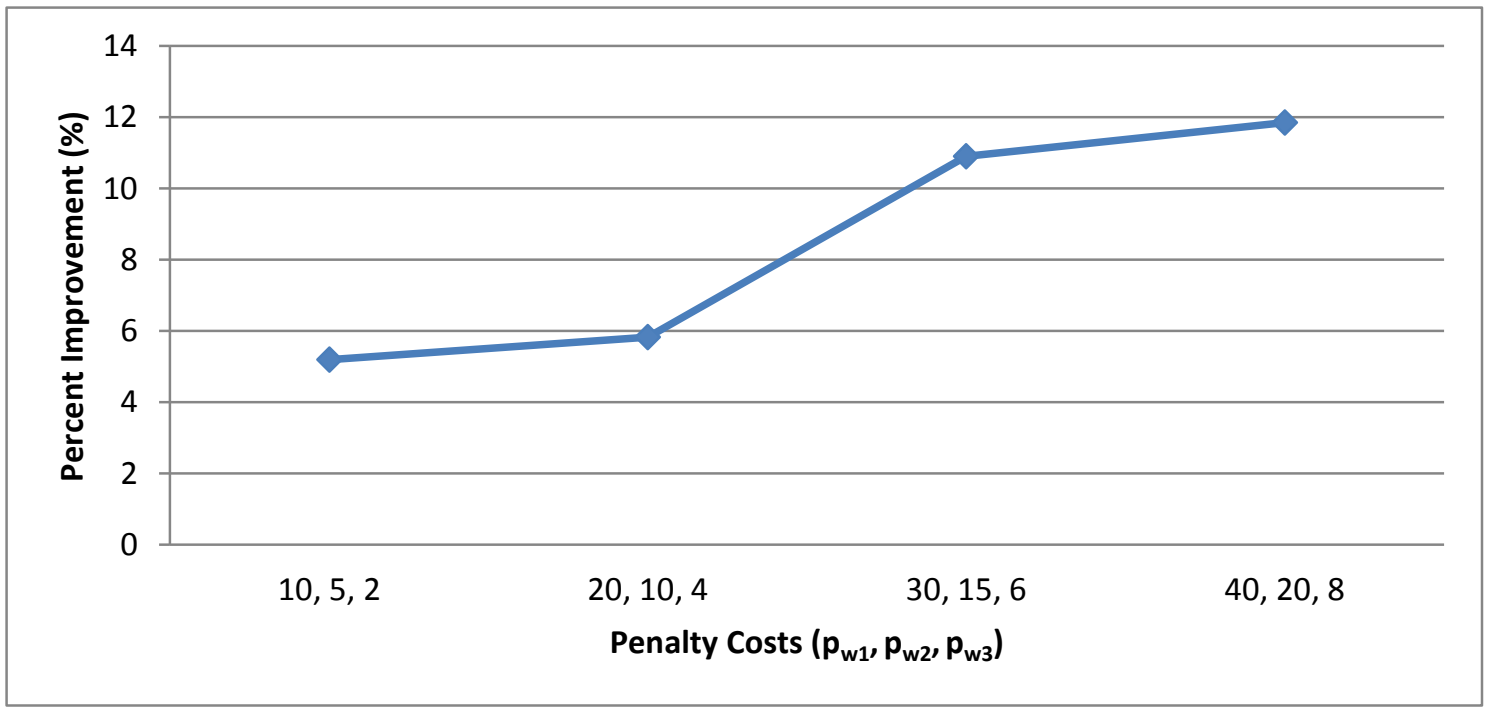

Figure 11. Percent improvement obtained by operation-dependent maintenance decisionmaking policy over the operation-independent CBM policy for different penalty costs 


\subsubsection{Influence of production goals $\left(\mathbf{N}_{\mathbf{w}_{\mathrm{j}}}\right)$}

Let us now assess the performance of the proposed methodology under increasingly demanding production goals (i.e. as one increases the production goals $N_{w_{j}}$ for each product type in the cost functions (1) and (2)). The expected profit and percent improvements of the newly proposed method over the benchmark operation-independent CBM policy for different production goals are given in Figure 12 and Figure 13, respectively (for corresponding numerical results, please see Appendix A.4). As can be seen in Figure 12, the operation-dependent CBM policy again yields higher expected profits when compared to the operation-independent CBM policy. In addition, although the expected profits obtained from the operation-dependent CBM policy decrease slightly as the production goals increase (which happens because the increasing production goals becomes less and less likely to be met), the profit decreases corresponding to the operation-independent CBM policy are even more dramatic.

The aforementioned results are also intuitively plausible. Namely, as the production goals increase, avoiding unscheduled downtime and production penalty costs by preventing the production of scrap wafers becomes even more important. The operation-dependent CBM policy allows operation-specific maintenance triggering states and thus offers more freedom in the maintenance decision-making, which ultimately leads to a higher likelihood of meeting the production goals. For example, for the operations that are executed in the completion of the wafers that have higher unmet production penalty costs, the operation-dependent maintenance decision-making policy 
can suggest earlier triggering of maintenance, thus reducing the number of scrap wafers and increasing the likelihood of making that production goal. It can also compensate for the consequent increase in the number of preventive maintenance events by suggesting later triggering of maintenance events for other operations. The traditional operationindependent $\mathrm{CBM}$ policy does not have these flexibilities and as the production goals increase, the gap between the number of successfully produced wafers and the production goal increases more dramatically than in the case of the operation-dependent maintenance decision-making policy. Therefore, it is logical that as the production goals increase, the relative benefits of the operation-dependent maintenance decision-making policy also increase, as can be seen in Figure 13.

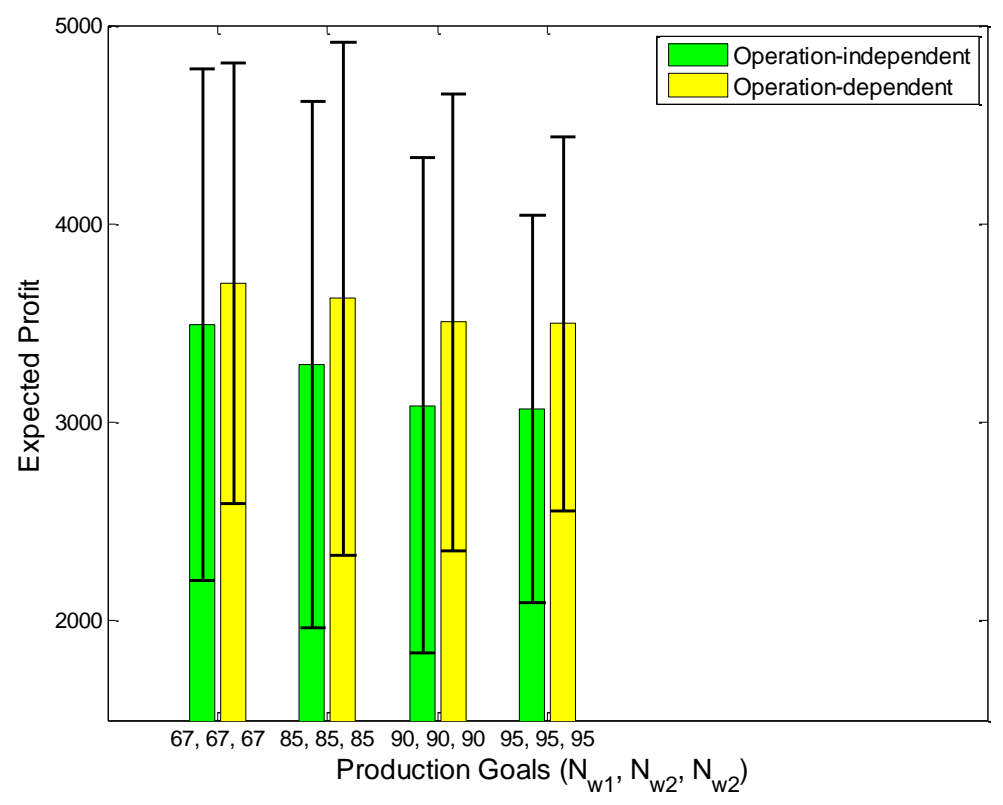

Figure 12. Comparison of expected profits for different production goals (black lines denote the $-/+2 \sigma$ control limits) 


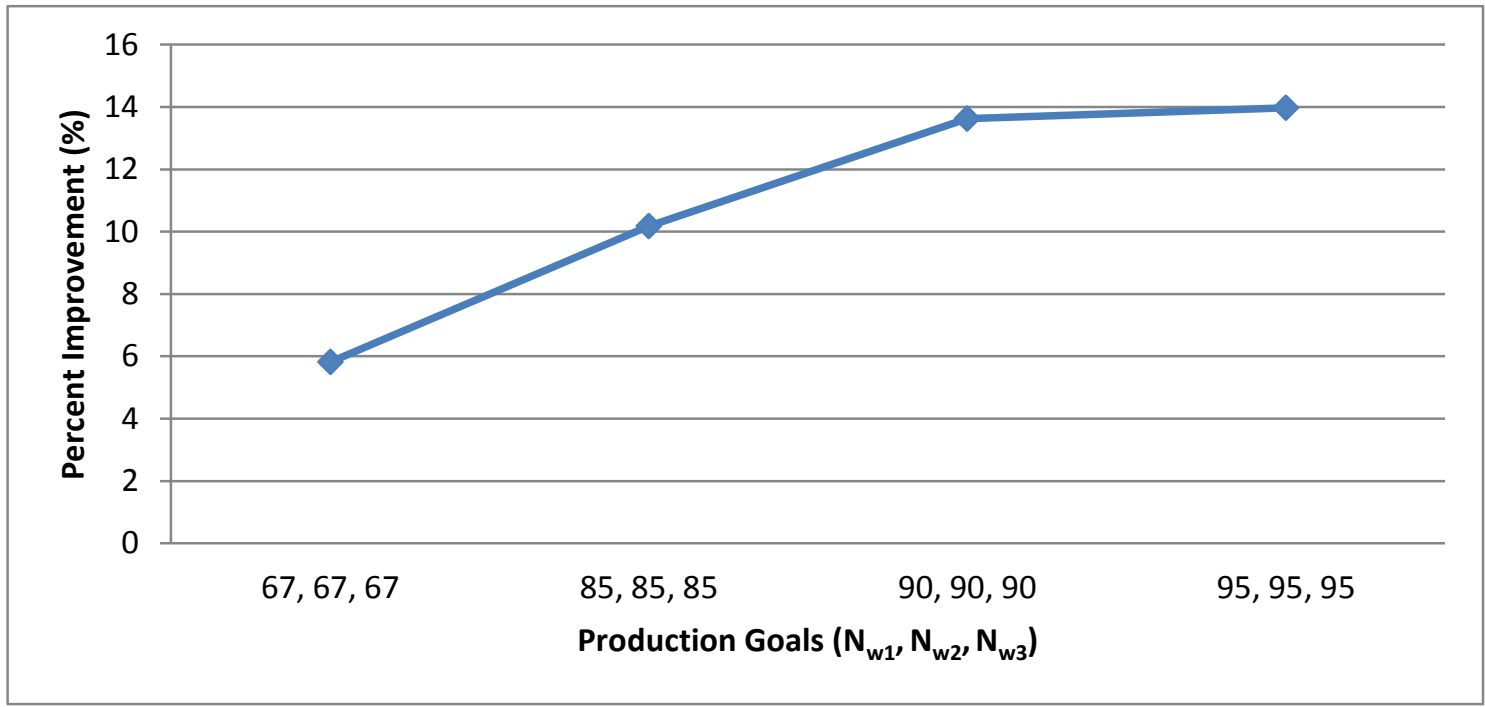

Figure 13. Percent improvement over the operation-independent CBM policy obtained by the operation-dependent maintenance decision-making policy for different production goals

\subsubsection{Influence of probabilities that preventive maintenance actions restored the perfect condition of the maintained system (MP)}

In this study, we acknowledge the fact that different workers may have different skill sets and hence the success of a maintenance operation may be different. As mentioned in the problem description in Section 3.2, we assume that not every maintenance operation may result in "good as new" state, i.e. imperfect maintenance may happen. In this section, we assessed the effects of maintenance personnel skills and effectiveness of maintenance on the expected profits by using various probabilities that the triggered PM event was perfect (i.e. that the PM event restored the condition of the maintained system to "as good as new"). A low probability of perfect maintenance 
intervention implies maintenance personnel executing PM interventions less effectively (possibly because of lower skill levels) and vice versa.

Various effectiveness levels of PM interventions are tested using different Markov transition matrices corresponding to condition-state recovery induced by PM. Figure 14 lists the PM-related Markov transition matrices tested in this study. Going from MP1 to MP5, we see increasingly efficient PM interventions, with increasing probabilities that PM returns the maintained system to better states (closer to the "as good as new" state). Note that MP5 corresponds to perfect maintenance operations that always return the maintained system to the "as good as new" state.

$$
\begin{gathered}
M P_{1}=\left[\begin{array}{ccccc}
0 & 0 & 0 & 0 & 0 \\
1 & 0 & 0 & 0 & 0 \\
0.20 & 0.80 & 0 & 0 & 0 \\
0.20 & 0.60 & 0.20 & 0 & 0 \\
1 & 0 & 0 & 0 & 0
\end{array}\right] \quad M P_{2}=\left[\begin{array}{ccccc}
0 & 0 & 0 & 0 & 0 \\
1 & 0 & 0 & 0 & 0 \\
0.40 & 0.60 & 0 & 0 & 0 \\
0.40 & 0.45 & 0.15 & 0 & 0 \\
1 & 0 & 0 & 0 & 0
\end{array}\right] \\
M P_{3}=\left[\begin{array}{ccccc}
0 & 0 & 0 & 0 & 0 \\
1 & 0 & 0 & 0 & 0 \\
0.60 & 0.40 & 0 & 0 & 0 \\
0.60 & 0.30 & 0.10 & 0 & 0 \\
1 & 0 & 0 & 0 & 0
\end{array}\right] \\
M P_{5}=\left[\begin{array}{lllll}
0 & 0 & 0 & 0 & 0 \\
1 & 0 & 0 & 0 & 0 \\
1 & 0 & 0 & 0 & 0 \\
1 & 0 & 0 & 0 & 0 \\
1 & 0 & 0 & 0 & 0
\end{array}\right]
\end{gathered}
$$

Figure 14. Perfect maintenance probabilities used for sensitivity analysis 
The expected profits obtained for various levels of maintenance efficacies are shown in Figure 15, while the percent improvements obtained with operation-dependent maintenance decision-making policy over the operation-independent CBM policy are given in Figure 16 (see Appendix A.5 for numerical values of the maintenance triggering state results).

Once again, one can observe from Figure 15 that for all levels of PM efficacy (PM characterized by Markov transition matrices MP1-MP5), the operation-dependent CBM policy results in a higher expected profit when compared to the benchmark operation-independent CBM policy. In addition, from Figure 16 one can see that higher levels of PM efficacy ${ }^{2}$ result in more pronounced percentual improvements of the newly proposed policy over the traditional operation-independent CBM policy. This can be explained by the fact that in sophisticated decision mechanisms, such as the operationdependent maintenance decision-making proposed in this study, the success of decisions depends on the effectiveness of the execution of those decisions. If the decisions are not executed effectively (when probabilities of restoring the good conditions of the maintained machine are lower), it could remove some of the benefits of the operationdependent decision policy.

${ }^{2}$ Situations when Markovian transition matrices corresponding to PMs have higher likelihood of returning the system to the "as good as new" state, i.e. scenarios obtained as we go from Markovian transition matrix $\mathrm{MP}_{1}$, towards $\mathrm{MP}_{2}$, then on to $\mathrm{MP}_{3}$ and so on. 


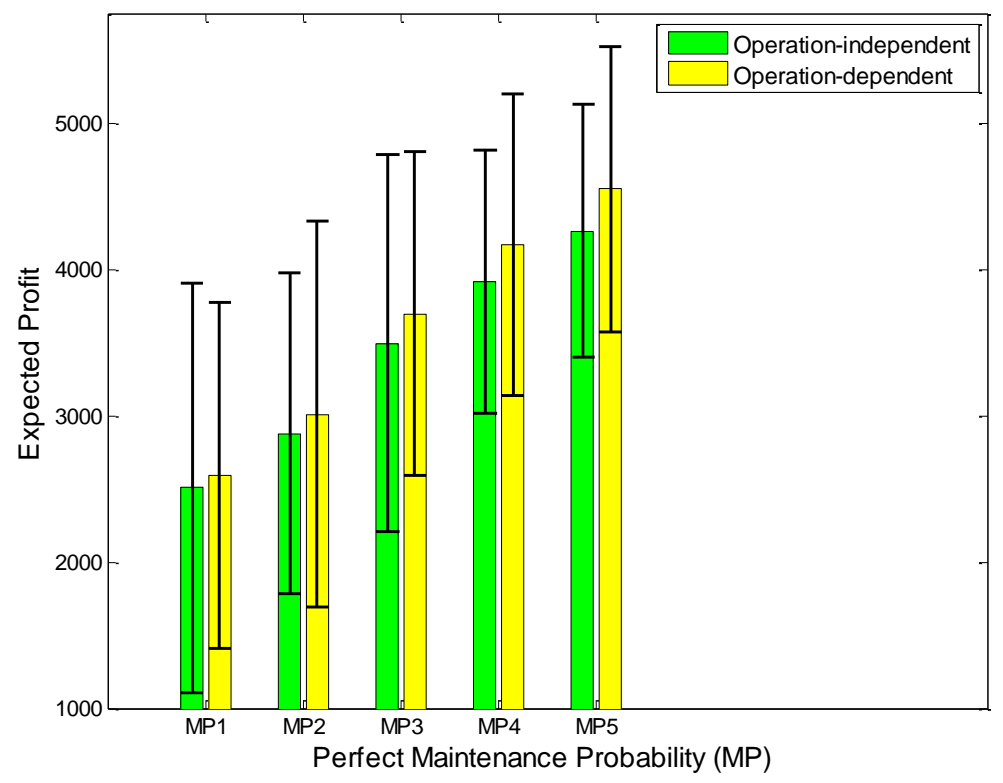

Figure 15. Comparison of expected profits for different perfect maintenance probabilities (black lines denote the $-/+2 \sigma$ control limits)

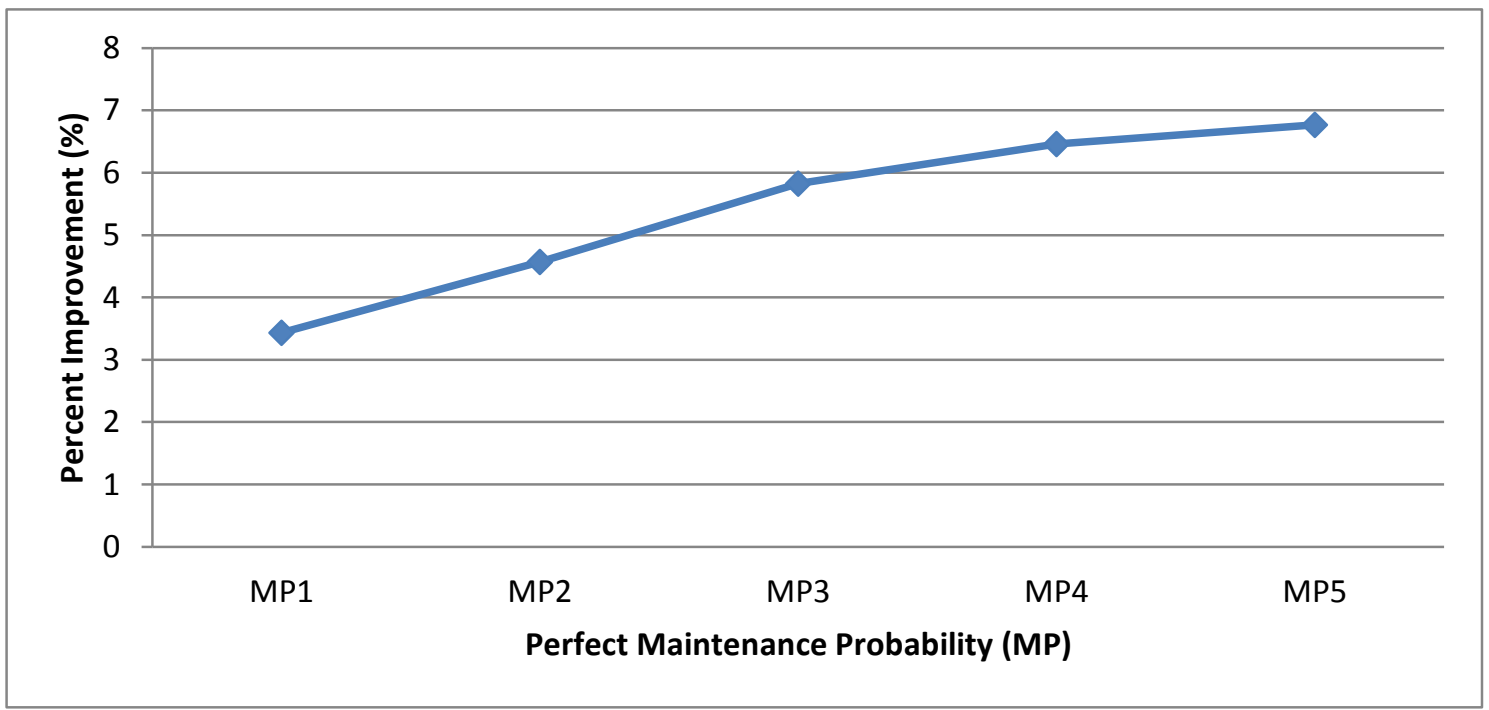

Figure 16. Percent improvement obtained by the operation-dependent maintenance decision-making policy over the operation-independent CBM policy for different perfect maintenance policies 


\subsection{Conclusions}

This chapter presents a methodology resulting in an operation-dependent maintenance scheduling policy for a manufacturing system composed of multiple manufacturing stations that can execute multiple operations. The new methodology is based on a metaheuristic optimization where candidate solutions are evaluated through repeated discrete event simulations of the underlying manufacturing system. The newly introduced operation-dependent decision making policy was evaluated and benchmarked against the traditional operation-independent CBM policy on an example of a cluster tool model, used routinely in semiconductor manufacturing industry. The new operationdependent CBM policy consistently outperformed the benchmark maintenance decisionmaking policy. Furthermore, a sensitivity analysis was performed and it was observed that higher reactive maintenance costs, unmet production penalty costs, production goals and efficacy of maintenance execution lead to more significant relative benefits of the newly proposed maintenance policy over the traditional operation-independent CBM policy. The aforementioned conclusions support the intuition that the new decisionmaking policy reduces unscheduled downtimes, decreases the number of scrap products and increases the likelihood of meeting the production goals through added freedom in the maintenance decision-making process. 


\section{Chapter 4}

\section{Integrated Maintenance and Operations Decision Making in Flexible Manufacturing Systems}

\subsection{Introduction ${ }^{3}$}

As mentioned in Section 3.1, machines in flexible manufacturing systems (FMS) have the capability of executing different operations and the degradation of a machine is highly dependent on the operation performed on it. Therefore, one can reconfigure production operations to increase the chance of successfully executing all operations and to decrease maintenance costs. For example, one could reroute operations from a degraded machine to less degraded ones to improve the yield and reduce the likelihood of unscheduled downtimes. In addition to that, one could also change sequence in which products are produced and delay scheduled maintenance to times when it is less intrusive on the overall production and most cost effective (weekends, or times of low demand).

\footnotetext{
${ }^{3}$ This chapter is based on the following three publications:

a. Celen, M., Djurdjanovic, D., 2012, "Joint Maintenance and Production Operations Decision Making in Flexible Manufacturing Systems," Proceedings of the ASME 2012 International Manufacturing Science and Engineering Conference, Paper ID. MSEC2012-7258.

M. Celen wrote this publication under D. Djurdjanovic's supervision.

b. Cholette, M. E., Celen, M., Djurdjanovic, D., and Rasberry, J. D., 2013, "Condition Based Monitoring and Operational Decision Making in Semiconductor Manufacturing," IEEE Transactions on Semiconductor Manufacturing, vol. 26, no. 4, pp. 454-464. M. Celen wrote Sections IV and V in this publication.

c. Celen, M., Djurdjanovic, D., 2015, "Integrated Maintenance Decision-Making and Product Sequencing in Flexible Manufacturing Systems," ASME Journal of Manuf. Sci. and Eng., vol. 137, no. 4, pp. 1-15.

M. Celen wrote this publication under D. Djurdjanovic's supervision.
} 
This section will illustrate benefits of joint maintenance and product dispatching in a FMS in which one knows operating mode dependent degradation dynamics.

In Chapter 3, the sequence in which different product types are produced was assumed to be a priori given and was not optimized. Unlike Chapter 3, in this chapter, we pursue an integrated decision-making policy for maintenance scheduling and product sequencing in a multiple-product/multiple-station manufacturing system, similar to the one considered in Chapter 3 (i.e. consisting of multiple manufacturing stations, each of which can execute a set of operations and each of which undergoes an observable operation-specific degradation process). The decision-making will be done by maximizing a customizable reward function, taking into account rewards of production, the costs of maintenance operations and penalties for unmet production goals.

The rest of this chapter is organized as follows. In Section 4.2, we formally define the problem of integrated decision-making for maintenance scheduling and production sequencing. In Section 4.3, we introduce a novel solution procedure for the problem defined in Section 4.2. The results collected from the simulation of a semiconductor cluster tool are enclosed in Section 4.4 in order to assess the performance of the newly proposed optimization methodology and compare it with traditional policies as well as the policy proposed in Chapter 3. Section 4.5 discusses the conclusions of this work and possible directions for future work. 


\subsection{Problem Statement}

As in Chapter 3, let us consider a FMS with $m$ manufacturing stations labeled $c_{1}, c_{2}, \ldots, c_{m}$, producing a set of product types $W=\left\{w_{1}, w_{2}, \ldots, w_{l}\right\}$. Let $O=$ $\left\{o_{1}, o_{2}, \ldots, o_{K}\right\}$ be the set of all operations that can be executed by the stations of that manufacturing system. Each product type $w_{j}, w_{j} \in W$, is associated with a sequence of operations $O_{w_{j}}=\left[o_{w_{j}}^{1}, o_{w_{j}}^{2}, \ldots, o_{w_{j}}^{p_{j}}\right]$, where $p_{j}$ is the number of operations needed to manufacture that product type. It is assumed that each station $c_{i}$ can execute operations $O_{c_{i}}=\left\{o_{c_{i}}^{1}, o_{c_{i}}^{2}, \ldots, o_{c_{i}}^{q_{i}}\right\} \subset O$, where $q_{i}$ is the number of operations that can be executed by that station. The goal will be to produce $N_{w_{j}}$ of each product type $w_{j}, w_{j} \in W$, within a certain mission time $T$.

The degradation process of each station will be modeled via concatenated operation-specific Markov models. More precisely, the degradation states of each station $c_{i}$ are assumed to be observable (or perfectly inferred) and follow operation-specific Markov models defined over a common state space $S_{c_{i}}=\left\{1,2, \ldots, M_{c_{i}}\right\}$ (degradation state "1" denoting the "good as new" state and $\mathrm{M}_{\mathrm{c}_{\mathrm{i}}}$ denoting the "failed" state). For any station, the probability of transitioning from state $a$ to state $b$ if operation $o_{k}$ is executed in it is defined as operation-dependent and station dependent $P_{a, b}^{\left(o_{k}\right)}$, which yields operationdependent state transition matrices $P^{\left(o_{k}\right)}=\left[P_{a, b}^{\left(o_{k}\right)}\right], a \in\left\{1,2, \ldots, M_{c_{i}}\right\}, b \in\left\{1,2, \ldots, M_{c_{i}}\right\}$. All Markov chains are assumed to be unidirectional, modeling the well-accepted intuition 
that the condition of a manufacturing station can only worsen over time, unless a maintenance operation is done.

When a maintenance operation is performed, the condition of a station will get better, but it may not necessarily go to the "good as new" state. For any station the probability of transitioning to a better state as a result of a maintenance operation is modeled by station dependent state transition matrices $M P=\left[P_{d, e}\right], d \in\left\{2, \ldots, M_{c_{i}}\right\}$, $e \in\left\{1,2, \ldots, M_{c_{i}}-1\right\}$. Thus, maintenance itself is modeled as a single-step Markov process.

In addition, we take into account the fact that as the degradation level of a station increases, the probability of success of any operation executed on that station decreases. We assume that for any station the probability of success of a given operation is a known function of the operation $o_{k} \in O$ and the station state $X_{i}$, denoted as $S_{c_{i}}\left(o_{k}, X_{i}\right)$.

Let $S_{c_{i}}^{o_{k}}$ denote the state of station $c_{i}$ that triggers preventive maintenance when operation $o_{k}$ is executed on it. It means that if the current station's degradation state $X_{i}(t)$ is greater than or equal to $S_{c_{i}}^{o_{k}}$, then operation $o_{k}$ could not be performed on that station, unless a maintenance operation is done on it beforehand.

Finally, let $\left[d_{1}, \ldots, d_{F}\right]$ denote the production sequence in which the products are processed in the FMS, where $d_{q} \in\left\{w_{1}, w_{2}, \ldots, w_{l}\right\}, q=1,2, \ldots, F$ and $F$ is the total 
amount of products that are fed into the manufacturing system ${ }^{4}$. In this chapter, it will be assumed that the FMS is such that the sequence $\left[d_{1}, \ldots, d_{F}\right]$ in which products are released into the FMS can also be changed. This means that the goal of producing a given number of units of each product type produced in a system could be achieved through multiple different sequences in which the units are released into it.

The decision-making objective will be to find a combined policy of maintenance triggering states across a system and product-type sequencing which maximizes a reward function that considers the benefits of production, costs of maintenance and penalties for unmet production goals. The benefits of production (rewards) come from selling the good products ${ }^{5}$. As the machines degrade, the probability of successfully completing an operation decreases, hence the number of scrap (not good) products increases, which makes it harder to meet the production goal. If the number of good products produced is not enough to meet the production goal, we assume we lose the customer that demanded those products and hence incur an unmet production goal penalty. In order to maximize the reward function, we would like to increase our rewards as much as possible and incur no penalty for unmet production goal. This is only possible by keeping the machines in the "good as new" state and thus mostly eliminating scrap products. However, this would mean maintaining the machines constantly, which would be very costly. To find the trade-off between the number of maintenance events executed and the number of scrap

\footnotetext{
${ }^{4}$ Effectively, $\left[d_{1}, \ldots, d_{F}\right]$ is the sequence in which the products are released into the system.

${ }^{5}$ Products of acceptable quality.
} 
products produced, we have included maintenance costs associated with each preventive and reactive maintenance events into our reward function. More formally, the decision optimization problem will be defined as follows. Let $N_{w_{j}}$ denote the production goal for product type $w_{j} \in\left\{w_{1}, w_{2}, \ldots, w_{l}\right\}$, and let $n_{w_{j}}$ denote the number of good products of type $w_{j}$ produced during mission time $T$. The integrated decision-making policy will be pursued through the optimization procedure

$$
\begin{aligned}
& \underset{\operatorname{maximize}}{\max }\left(\sum_{j=1}^{l} R_{w_{j}} n_{w_{j}}-V_{r} c_{r}-V_{p} c_{p}-V_{c r} c_{c r}-V_{c p} c_{c p}-\sum_{j=1}^{l} a_{w_{j}}\left(N_{w_{j}}-n_{w_{j}}\right) p_{w_{j}}\right) \\
& {\left[\begin{array}{ccccc}
\left.S_{d_{1}, d_{2}, \ldots, d_{F}}^{o_{1}}\right] & S_{c_{2}}^{o_{1}} & \cdots & S_{c_{m}}^{o_{1}} \\
S_{c_{1}}^{o_{2}} & S_{c_{2}}^{o_{2}} & \cdots & S_{c_{m}}^{o_{2}} \\
\vdots & \vdots & \ddots & \vdots \\
S_{c_{1}}^{o_{K}} & S_{c_{2}}^{o_{K}} & \cdots & S_{c_{m}}^{o_{K}}
\end{array}\right]}
\end{aligned}
$$

where

- $R_{w_{j}}$ is the unit reward for good products produced of type $w_{j}, w_{j} \in W$

- $p_{w_{j}}$ is the unit penalty for penalizing unmet production for product type $w_{j}, w_{j} \in W$

- $c_{r}$ denotes the unit cost of reactive maintenance (RM) per reactive maintenance event

- $c_{p}$ is the unit cost of preventive maintenance (PM) per preventive maintenance event 
- $c_{c r}$ denotes the unit cost of RM conducted during time intervals in which maintenance operation can be performed at a cheaper price (such as night shifts, weekends $)^{6}$

- $c_{c p}$ is the unit cost of PM executed during time intervals in which maintenance operations can be executed at a cheaper price ${ }^{7}$

- $V_{r}$ is the total number of RM events

- $V_{p}$ is the total number of PM events

- $V_{c r}$ is the total number of RM events during less expensive maintenance time periods

- $V_{c p}$ is the total number of PM events during less expensive maintenance time periods

- $a_{w_{j}}=\left\{\begin{array}{l}1, \text { if } n_{w_{j}}<N_{w_{j}} \\ 0, \text { otherwise }\end{array}\right.$ for $\forall w_{j} \in W$

The objective function shown in (3) aims to find the product sequence $\left[d_{1}, d_{2}, \ldots, d_{F}\right]$ and operation-dependent maintenance triggering states $S_{c_{i}}^{o_{k}}$ that maximize the expected reward function in (3). We understand that cost models in different companies would be very different. Even within a single company, different parts of the company may implement different cost models. Therefore, we created a method that is not dependent on the cost function in the sense that we used simulation based

\footnotetext{
${ }^{6}$ Of course, if such periods of cheaper maintenance do not exist in the given FMS, then $c_{c r}=c_{r}$.

${ }^{7}$ Once again, if such periods of time do not exist in a given FMS, then $c_{c p}=c_{p}$.
} 
metaheuristic optimization where the cost function can easily be changed to suit the reality of a company while the optimization method would not change at all. In order to demonstrate our method, we utilized a very simple and intuitive objective function (shown in (3)) that rewards production and punishes downtime and scrap products.

The expectation operator is applied because of the stochastic effects induced by randomness in the reliability of equipment (states of manufacturing stations degrade according to operation specific and machine specific stochastic processes), conditiondependent yields (for each operation and each machine, one has a probability of successfully completing the operation), maintenance effects (equipment condition after maintenance is stochastic as mentioned earlier) and repair times (repair times are not deterministic in our study). All these random effects are modeled via discrete event simulations and objective function results obtained from multiple runs of the simulations are averaged to estimate the expected profits corresponding to any given product sequence and maintenance triggering policy.

In order to assess the performance of the proposed methodology, the integrated decision-making results will be compared with the traditional Operation-Independent CBM Policy, which assumes that the maintenance triggering states for each station are independent of the operation executed in it (i.e. $S_{c_{i}}^{o_{1}}=S_{c_{i}}^{o_{2}}=\cdots=S_{c_{i}}^{o_{K}}=S_{c_{i}}$ for each station $c_{i}$ ). The new decision-making policy will also be compared to the OperationDependent CBM Policy, presented in Chapter 3. In both of these benchmark problems, the product type sequence $\left[d_{1}, d_{2}, \ldots, d_{F}\right]$ is assumed to be a priori given, which is 
consistent with the traditional, fragmented approach to maintenance planning and production scheduling. For completeness, these two problems are defined below.

Operation-Independent CBM Policy Determination:

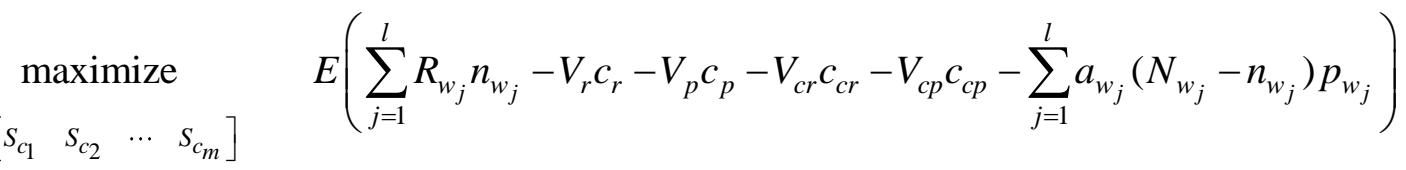

Operation-Dependent CBM Policy Determination:

$$
\begin{gathered}
\operatorname{maximize} \\
{\left[\begin{array}{cccc}
S_{c_{1}}^{o_{1}} & S_{c_{2}}^{o_{1}} & \cdots & S_{c_{m}}^{o_{1}} \\
S_{c_{1}}^{o_{2}} & S_{c_{2}}^{o_{2}} & \cdots & S_{c_{m}}^{o_{2}} \\
\vdots & \vdots & \ddots & \vdots \\
S_{c_{1}}^{o_{K}} & S_{c_{2}}^{o_{K}} & \cdots & S_{c_{m}}^{o_{K}}
\end{array}\right]}
\end{gathered}
$$

The integrated decision-making policy and both benchmark CBM policies are stationary in the sense that the selected maintenance triggering states and product type sequence would not change throughout the execution of all production.

\subsection{Solution Procedure}

For an $m$-station manufacturing system with $K$ operations that can be executed in these stations, and producing $F$ products, there are up to $\prod_{i=1}^{m}\left(M_{c_{i}}-1\right)^{K} \times \frac{F !}{\prod_{j=1}^{l} F_{w_{j}} !}$ candidate solutions in the solution space, though it can be decreased by acknowledging 
that not all operations can be executed in all stations. Nevertheless, even the aforementioned reduction usually results in a solution space that is too large for complete enumeration, especially when candidate solutions are evaluated via replicated discreteevent simulations.

Hence, in order to find a practical, applicable and near optimal solution, a Tabu Search algorithm [104] is used, with discrete-event simulations of the target manufacturing system providing cost effects of any candidate solution. The average profit relevant to each candidate solution is evaluated from multiple replications of the discreteevent simulations of the target manufacturing system and is fed back into the Tabu Search algorithm to guide the movements towards improved candidate decisions.

The local neighborhood of any candidate solution is generated by generating neighborhoods for the candidate maintenance triggering states and product type sequence. To generate the neighborhood for maintenance triggering states, a cell in the matrix representing the current solution (rows representing operations and columns representing machines) is selected randomly and its value is perturbed. The details of maintenance triggering state neighborhood generation can be found in Section 3.3.1.2. For the product type sequence, neighbors were generated by pairwise interchanges of two randomly selected product types. An example of product type sequence neighborhood generation for a system that produces 10 products ( 3 of product type $w_{1}, 2$ of product type $w_{2}, 2$ of product type $w_{3}$ and 3 of product type $\left.w_{4}\right)$ is given in Figure 17 . At each iteration, for the current CBM and product type sequence solution, a limited number of 
product type sequence neighbors are generated. For the sequence that gives the highest profit, a predetermined number of cells are selected from the matrix representing the maintenance triggering states and their values are perturbed. The operation-dependent CBM policy presented in Chapter 3 and a randomly selected product type sequence were used in this study as the initial solution in order to speed up the metaheuristic optimization process.

Please note that the solution procedures for the benchmark policies have been presented in detail in Chapter 3, and therefore will not be elaborated on in this chapter.

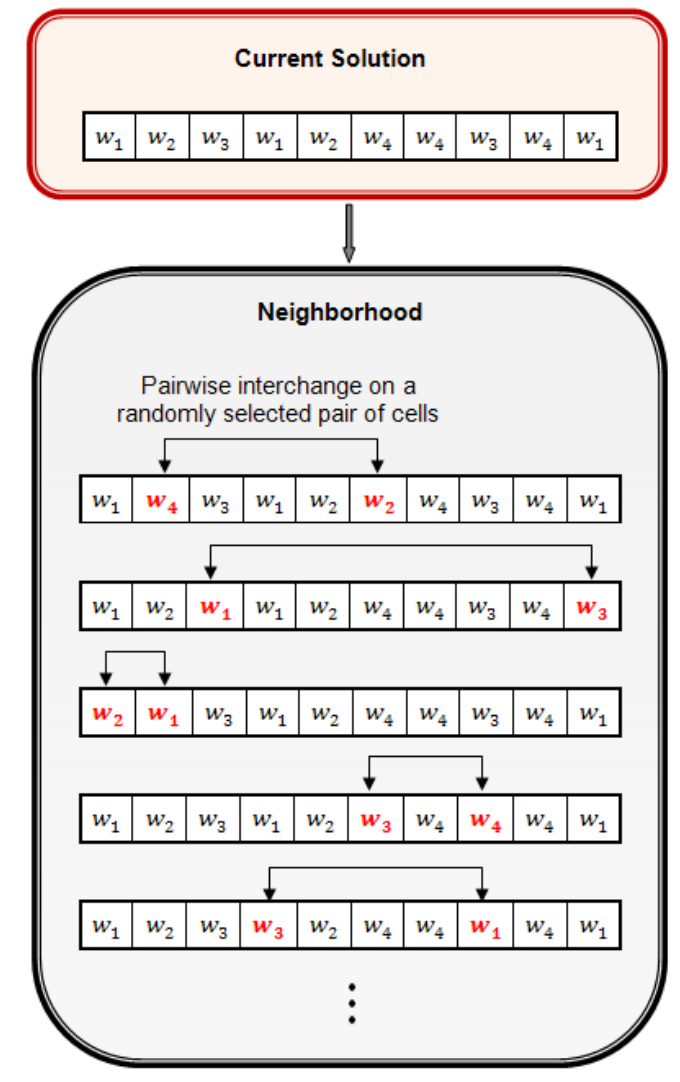

Figure 17. Neighborhood generation for product type sequence 


\subsection{Results}

Integrated decision-making of preventive maintenance scheduling and product sequencing was tested on an example of a cluster tool, described in Section 3.4.

In order to assess the performance of the newly proposed optimization methodology, the AutoMod software package [140] was used to simulate a 5-chamber cluster tool, producing 3 types of wafers. Throughout our study, 40 simulation replications were used to evaluate each candidate solution for a maintenance and product sequencing policy. This number was determined by increasing the number of replications until further increase of this number did not result in significant changes in average profit effects of simulations ran for a wide set of parameters. There was no control over the random number seeds that were used in these replications. It also should be noted that, at the end of each simulation, the current system condition is not maintained.

\subsubsection{Baseline Example}

The parameters used to generate the results are given in Table 4, Table 5 and Figure 18. Within this plethora of parameters, let us note that PM times are assumed to be approximately one half of the corresponding RM times. In addition, the night shifts (8pm

- 8am) were assumed to be the periods of cheaper maintenance, during which maintenance events were assumed to $\cos t 2 / 5^{\text {th }}$ of a regular maintenance event executed during day shifts $(8 \mathrm{am}-8 \mathrm{pm})$, for both preventive and reactive maintenance interventions. It can also be noted that, based on the corresponding Markovian state 
transition matrices, operations $o_{2}, o_{3}, o_{7}, o_{9}, o_{11}$ and $o_{12}$ degrade the chambers slower than other operations. In addition, analysis of degradation dependent yield shows that operations $o_{3}, o_{7}, o_{9}, o_{11}$ and $o_{13}$ are more sensitive to degradation, since their yields decrease more severely as the degradation state of the chamber increases. These points will be important as we analyze the effects of the newly introduced integrated decisionmaking policy and compare them to the benchmark policies.

The operation-independent CBM policy was determined through complete enumeration and $\mathrm{S}_{\mathrm{CBM}}=\left[\begin{array}{lllll}3 & 3 & 2 & 3 & 2\end{array}\right]$ was found to be the best operation-independent CBM policy in the baseline parameter setting. On the other hand, in order to optimize the integrated decision-making policy, Tabu Search algorithm described in Section 3.3.1.2 was used. The parameters of the tabu search algorithm used in this study were selected in an ad hoc manner and are given in Table 6.

From Figure 19, it is immediately visible that the integrated decision-making policy results in a higher expected profit, when compared to the traditional operationindependent CBM policy and the operation-dependent CBM policy introduced in Chapter 3. There are several reasons for this performance improvement brought by the newly proposed decision-making method. Firstly, the benefits of considering operationdependent degradation dynamics and postulating an operation-dependent maintenance policy were apparent, similarly to what we saw in Chapter 3. Namely, for the best integrated decision-making policy, it was observed that the maintenance triggering states $S_{c_{4}}^{o_{2}}, S_{c_{3}}^{o_{3}}, S_{c_{4}}^{o_{8}}, S_{c_{3}}^{o_{9}}, S_{c_{2}}^{o_{10}}$ and $S_{c_{5}}^{o_{11}}$ are higher (allowing more degradation) compared to 
the traditional, operation-independent CBM policy. As mentioned before, operations $O_{2}$ and $o_{3}$ degrade the chambers slower than others and the optimized operation-mode dependent maintenance triggering states yielded by the procedure proposed in this chapter support the intuition that less frequent maintenance should be allowed for slower degrading operations. In addition, since operations $o_{8}, o_{9}, o_{10}$ and $o_{11}$ are involved in the manufacturing of cheaper (less rewarding) wafers, the corresponding optimized maintenance triggering states also conform with the intuition that as the wafer reward decreases, the effect of completing that wafer successfully on the profit decreases, thus favoring later triggering of maintenance.

In addition, clear benefits of the ability to adjust product sequencing within the newly proposed decision-making scheme can be seen in Figure 20. It can be observed in Figure 20 (a) that the integrated decision-making policy not only decreases the total number of PM events, but also increases the number of the PM events occurring in the cheaper maintenance periods, when compared to the benchmark policies. The reason for this is that, by allowing changes in the product sequence, the integrated decision-making policy takes advantage of the cheaper maintenance periods and rearranges the product sequencing in a way that more PM events are pushed to take place during those periods. This is a significant feature which contributed to the beneficial cost effects of the decision-making procedure introduced in this study, which is not available in the fragmented maintenance and product sequencing decision-making schemes traditionally pursued in manufacturing today. 
Table 4. Summary of simulation parameters for integrated decision-making

\begin{tabular}{|c|c|}
\hline Set of chambers & $C=\left\{c_{1}, c_{2}, \ldots, c_{5}\right\}$ \\
\hline Wafer types & $W=\left\{w_{1}, w_{2}, w_{3}\right\}$ \\
\hline $\begin{array}{l}\text { Number of wafers for each } \\
\text { wafer type }\end{array}$ & $\begin{array}{l}F=12, F_{w_{1}}=4, F_{w_{2}}=4, F_{w_{3}}=4 \text { (These are the number of lots and each } \\
\text { lot consists of } 25 \text { wafers, hence } 100 \text { wafers of each wafer type are fed into } \\
\text { the system) }\end{array}$ \\
\hline Wafer type sequence & {$\left[d_{1}, d_{2}, \ldots, d_{12}\right]=\left[w_{1}, w_{2}, w_{3}, w_{1}, w_{2}, w_{3}, w_{1}, w_{2}, w_{3}, w_{1}, w_{2}, w_{3}\right]$} \\
\hline Production goal (wafers) & $N_{w_{1}}=N_{w_{2}}=N_{w_{3}}=85$ \\
\hline Set of operations & $O=\left\{o_{1}, o_{2}, \ldots, o_{13}\right\}$ \\
\hline Operation cycle times (min) & {$[10,20,15,15,10,20,20,10,25,10,15,10,10]$} \\
\hline $\begin{array}{l}\text { Operation sequences for each } \\
\text { wafer type }\end{array}$ & $\begin{array}{l}O_{w_{1}}=\left[o_{1}, o_{2}, o_{3}, o_{4}, o_{13}, o_{5}\right] \\
O_{w_{2}}=\left[o_{6}, o_{3}, o_{7}, o_{11}, o_{12}\right] \\
O_{w_{3}}=\left[o_{8}, o_{9}, o_{10}, o_{11}, o_{6}\right]\end{array}$ \\
\hline $\begin{array}{l}\text { Operations executable in each } \\
\text { chamber }\end{array}$ & $\begin{array}{l}O_{c_{1}}=\left\{o_{1}, o_{2}, o_{4}, o_{5}, o_{6}, o_{7}, o_{8}, o_{9}\right\} \\
O_{c_{2}}=\left\{o_{1}, o_{2}, o_{3}, o_{4}, o_{6}, o_{8}, o_{9}, o_{10}\right\} \\
O_{c_{3}}=\left\{o_{3}, o_{4}, o_{5}, o_{7}, o_{9}, o_{10}, o_{11}\right\} \\
O_{c_{4}}=\left\{o_{2}, o_{8}, o_{12}, o_{13}\right\} \\
O_{c_{5}}=\left\{o_{1}, o_{5}, o_{6}, o_{7}, o_{11}, o_{12}\right\}\end{array}$ \\
\hline Degradation state space & $S_{c_{1}}=S_{c_{2}}=\cdots=S_{c_{5}}=\{1,2, \ldots, 5\}$ \\
\hline $\begin{array}{l}\text { Distributions of preventive } \\
\text { maintenance times for each } \\
\text { chamber ( } \mathrm{min})\end{array}$ & {$[U(31,49), U(51,69), U(41,59), U(21,39), U(21,39)]$} \\
\hline Periods of cheaper maintenance & 8:00pm - 8:00am each day \\
\hline $\begin{array}{l}\text { Reactive maintenance times for } \\
\text { each chamber ( } \mathrm{min})\end{array}$ & {$[U(62,98), U(102,138), U(82,118), U(42,78), U(42,78)]$} \\
\hline Reward parameters (\$) & $R_{w_{1}}=50, R_{w_{2}}=25, R_{w_{3}}=10$ \\
\hline Costs (\$) & $c_{p}=50, c_{r}=250, c_{c p}=20, c_{c r}=100, p_{w_{1}}=20, p_{w_{2}}=10, p_{w_{3}}=4$ \\
\hline
\end{tabular}


Table 5. Probability of success for each operation for integrated decision-making

\begin{tabular}{|l|ccccc|}
\cline { 2 - 6 } \multicolumn{1}{c|}{} & State 1 & State 2 & State 3 & State 4 & State 5 \\
\hline$o_{1}$ & 1 & 0.9606 & 0.8295 & 0.8122 & 0.7938 \\
\hline$o_{2}$ & 1 & 0.9900 & 0.9327 & 0.9132 & 0.8896 \\
\hline$o_{3}$ & 1 & 0.9224 & 0.6022 & 0.5677 & 0.4935 \\
\hline$o_{4}$ & 1 & 0.9801 & 0.8877 & 0.8619 & 0.8284 \\
\hline$o_{5}$ & 1 & 0.9512 & 0.8650 & 0.7643 & 0.7438 \\
\hline$o_{6}$ & 1 & 0.9276 & 0.9110 & 0.8946 & 0.8525 \\
\hline$o_{7}$ & 1 & 0.9317 & 0.8386 & 0.5721 & 0.5062 \\
\hline$o_{8}$ & 1 & 0.9631 & 0.8301 & 0.7737 & 0.7316 \\
\hline$o_{9}$ & 1 & 0.9360 & 0.9042 & 0.7430 & 0.6866 \\
\hline$o_{10}$ & 1 & 0.9511 & 0.9397 & 0.9284 & 0.8991 \\
\hline$o_{11}$ & 1 & 0.9500 & 0.8758 & 0.8418 & 0.7084 \\
\hline$o_{12}$ & 1 & 0.9739 & 0.9605 & 0.8880 & 0.8603 \\
\hline$o_{13}$ & 1 & 0.9276 & 0.7848 & 0.7169 & 0.6354 \\
\hline
\end{tabular}

$$
\begin{gathered}
P^{\left(o_{1}\right)}=\left[\begin{array}{ccccc}
0.98 & 0.02 & 0 & 0 & 0 \\
0 & 0.98 & 0.02 & 0 & 0 \\
0 & 0 & 0.98 & 0.02 & 0 \\
0 & 0 & 0 & 0.98 & 0.02 \\
0 & 0 & 0 & 0 & 1
\end{array}\right] P^{\left(o_{2}\right)}=\left[\begin{array}{ccccc}
0.90 & 0.08 & 0.02 & 0 & 0 \\
0 & 0.90 & 0.08 & 0.02 & 0 \\
0 & 0 & 0.90 & 0.08 & 0.02 \\
0 & 0 & 0 & 0.93 & 0.07 \\
0 & 0 & 0 & 0 & 1
\end{array}\right] P^{\left(o_{3}\right)}=\left[\begin{array}{ccccc}
0.93 & 0.05 & 0.02 & 0 & 0 \\
0 & 0.93 & 0.05 & 0.02 & 0 \\
0 & 0 & 0.93 & 0.05 & 0.02 \\
0 & 0 & 0 & 0.97 & 0.03 \\
0 & 0 & 0 & 0 & 1
\end{array}\right] \\
P^{\left(o_{4}\right)}=\left[\begin{array}{ccccc}
0.94 & 0.04 & 0.015 & 0.005 & 0 \\
0 & 0.94 & 0.04 & 0.015 & 0.005 \\
0 & 0 & 0.94 & 0.04 & 0.02 \\
0 & 0 & 0 & 0.94 & 0.06 \\
0 & 0 & 0 & 0 & 1
\end{array}\right] \quad P^{\left(o_{5}\right)}=\left[\begin{array}{ccccc}
0.98 & 0.015 & 0.005 & 0 & 0 \\
0 & 0.98 & 0.015 & 0.005 & 0 \\
0 & 0 & 0.98 & 0.015 & 0.005 \\
0 & 0 & 0 & 0.98 & 0.02 \\
0 & 0 & 0 & 0 & 1
\end{array}\right]
\end{gathered}
$$$$
P^{\left(o_{6}\right)}=\left[\begin{array}{ccccc}
0.95 & 0.04 & 0.01 & 0 & 0 \\
0 & 0.95 & 0.04 & 0.01 & 0 \\
0 & 0 & 0.95 & 0.04 & 0.01 \\
0 & 0 & 0 & 0.95 & 0.05 \\
0 & 0 & 0 & 0 & 1
\end{array}\right] \quad P^{\left(o_{7}\right)}=\left[\begin{array}{ccccc}
0.93 & 0.04 & 0.02 & 0.01 & 0 \\
0 & 0.93 & 0.04 & 0.02 & 0.01 \\
0 & 0 & 0.93 & 0.06 & 0.01 \\
0 & 0 & 0 & 0.97 & 0.03 \\
0 & 0 & 0 & 0 & 1
\end{array}\right] \quad P^{\left(o_{8}\right)}=\left[\begin{array}{ccccc}
0.97 & 0.03 & 0 & 0 & 0 \\
0 & 0.97 & 0.03 & 0 & 0 \\
0 & 0 & 0.97 & 0.03 & 0 \\
0 & 0 & 0 & 0.97 & 0.03 \\
0 & 0 & 0 & 0 & 1
\end{array}\right]
$$$$
P^{\left(o_{9}\right)}=\left[\begin{array}{ccccc}
0.90 & 0.05 & 0.03 & 0.02 & 0 \\
0 & 0.90 & 0.05 & 0.03 & 0.02 \\
0 & 0 & 0.90 & 0.07 & 0.03 \\
0 & 0 & 0 & 0.90 & 0.10 \\
0 & 0 & 0 & 0 & 1
\end{array}\right] P^{\left(o_{10}\right)}=\left[\begin{array}{ccccc}
0.99 & 0.01 & 0 & 0 & 0 \\
0 & 0.99 & 0.01 & 0 & 0 \\
0 & 0 & 0.99 & 0.01 & 0 \\
0 & 0 & 0 & 0.99 & 0.01 \\
0 & 0 & 0 & 0 & 1
\end{array}\right] P^{\left(o_{11}\right)}=\left[\begin{array}{ccccc}
0.89 & 0.07 & 0.03 & 0.01 & 0 \\
0 & 0.89 & 0.07 & 0.03 & 0.01 \\
0 & 0 & 0.91 & 0.07 & 0.02 \\
0 & 0 & 0 & 0.91 & 0.09 \\
0 & 0 & 0 & 0 & 1
\end{array}\right]
$$$$
P^{\left(o_{12}\right)}=\left[\begin{array}{ccccc}
0.92 & 0.06 & 0.02 & 0 & 0 \\
0 & 0.92 & 0.06 & 0.02 & 0 \\
0 & 0 & 0.92 & 0.06 & 0.02 \\
0 & 0 & 0 & 0.95 & 0.05 \\
0 & 0 & 0 & 0 & 1
\end{array}\right] \quad P^{\left(o_{13}\right)}=\left[\begin{array}{ccccc}
0.95 & 0.03 & 0.02 & 0 & 0 \\
0 & 0.95 & 0.03 & 0.02 & 0 \\
0 & 0 & 0.95 & 0.03 & 0.02 \\
0 & 0 & 0 & 0.96 & 0.04 \\
0 & 0 & 0 & 0 & 1
\end{array}\right] \quad M P=\left[\begin{array}{ccccc}
0 & 0 & 0 & 0 & 0 \\
1 & 0 & 0 & 0 & 0 \\
0.60 & 0.40 & 0 & 0 & 0 \\
0.60 & 0.30 & 0.10 & 0 & 0 \\
1 & 0 & 0 & 0 & 0
\end{array}\right]
$$

Figure 18.Operation-specific transition probability matrices and maintenance transition probability matrix for integrated decision making 
Table 6. Parameters of tabu search for integrated decision-making

\begin{tabular}{|l|l|}
\hline Parameters of Tabu Search & Value \\
\hline Maximum iterations & 20 \\
\hline Tabu list length & 5 \\
\hline Neighbors for product type sequence & 10 \\
\hline Neighbors for maintenance triggering states & 15 \\
\hline Replication number to find expected profit & 40 \\
\hline
\end{tabular}

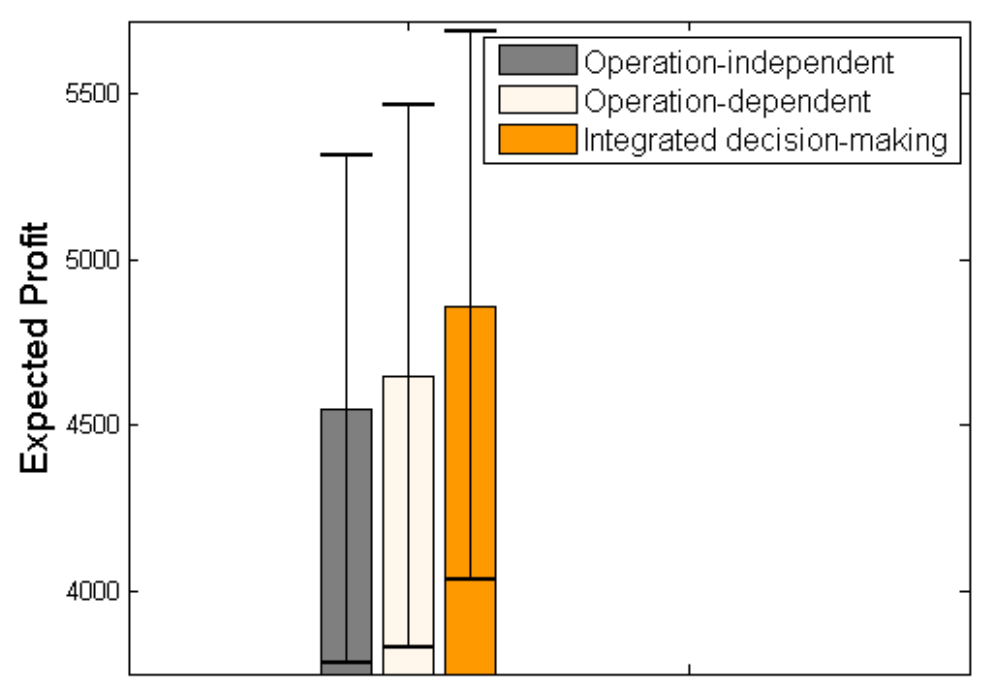

Figure 19. Expected profit comparison for integrated decision-making policy 


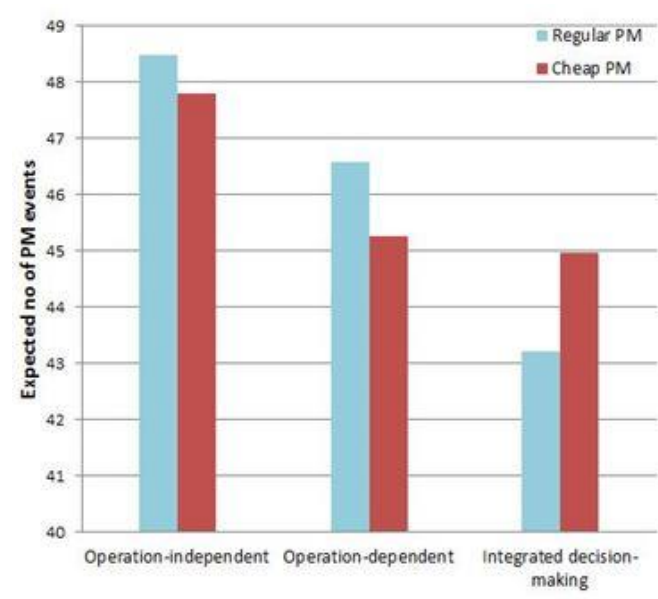

(a) Expected number of PM events

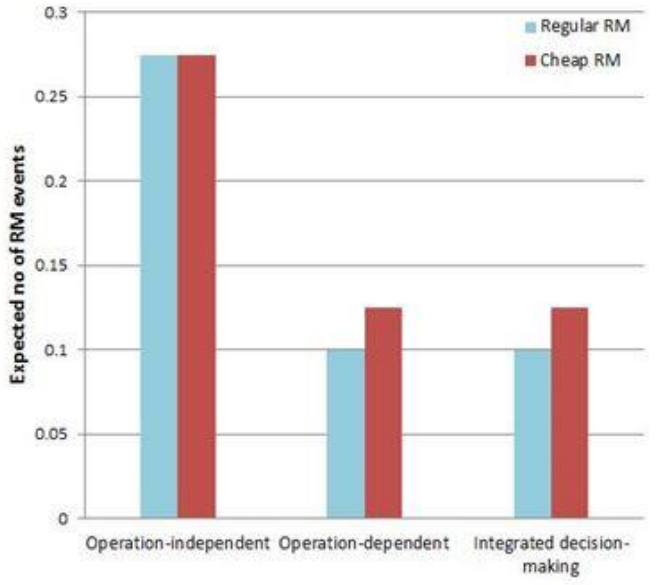

(b) Expected number of RM events

Figure 20. Comparison between the expected numbers of maintenance events conducted during the expensive and cheap maintenance periods

To gain better understanding of the newly proposed integrated decision-making policy, we studied the influences of cheap/regular maintenance cost ratio, unmet production penalty cost $\left(p_{w_{j}}\right)$ and production goal $\left(N_{w_{j}}\right)$ parameters, as well as the efficacy of maintenance execution on the resulting decisions and their effects. Results of these studies are enclosed below.

\subsubsection{Influence of cheap/regular maintenance cost ratio}

The ratios of maintenance costs during the cheap and regular maintenance periods were varied across a wide range of values ${ }^{8}$ and, as can be clearly observed from Figure 21, for all cheap/regular maintenance cost ratios, the integrated decision-making policy

\footnotetext{
${ }^{8}$ Regardless of the cheap/regular maintenance ratio, average maintenance costs over the length of the day were kept the same as they were for the baseline example.
} 
results in a higher expected profit when compared to the benchmark policies. Figure 22 illustrates how the integrated decision-making policy took advantage of the cheaper maintenance periods and delivered higher expected profits. Namely, as the cheap/regular maintenance cost ratio decreases and execution of PM operations during cheaper maintenance periods carries more potentials for savings, the integrated maintenance policy arranges the product sequence in such a way that more maintenance events occur during the cheaper maintenance periods, thus taking advantage of the reduced cost. This increasing trend is clearly evident in Figure 22.

Furthermore, as illustrated in Figure 23, the benefits of the integrated decisionmaking policy relative to the operation-independent $\mathrm{CBM}$ policy increase as the cheap/regular maintenance cost ratio decreases. This is reasonable since with the decrease in the cheap/regular maintenance cost ratio, the cost opportunity to push more maintenance events to the cheaper maintenance periods grows. Since only the integrated decision-making policy has the capability to, when necessary, re-sequence products and push more PM events to the cheaper maintenance periods, its cost relative benefits over the benchmark policies grow.

The p-values calculated through a paired t-test to observe the statistical significance of the outperformance of the proposed method over the benchmark policies are given in Table 7. The proposed integrated decision-making policy outperforms the operation-independent CBM policy with statistical significance in all instances. For the higher cheap/regular maintenance cost ratios, the benefits of the integrated decision- 
making policy over the operation-dependent CBM policy will be lower as pushing the maintenance events to cheaper maintenance periods would not yield substantial maintenance cost decrease. Therefore the proposed method does not outperform the operation-dependent CBM policy significantly for higher cheap/regular maintenance cost ratios.

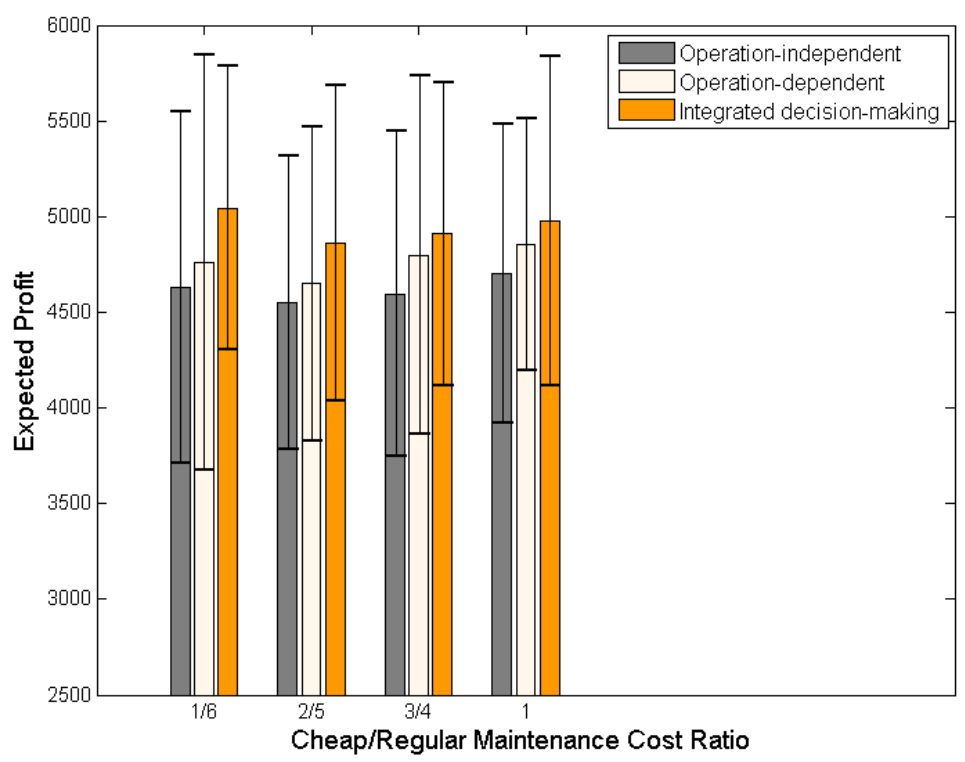

Figure 21. Comparison of expected profits for different cheap/regular maintenance cost ratios (black lines denote the $-/+2 \sigma$ limits of the simulation outcomes) for the integrated decision-making policy, operation-dependent CBM policy and the traditional operationindependent CBM policy

Table 7. $\mathrm{p}$-values for different cheap/regular maintenance cost ratios

\begin{tabular}{|c|c|c|c|c|}
\cline { 2 - 4 } \multicolumn{1}{c|}{} & $1 / 6$ & $2 / 5$ & $3 / 4$ & 1 \\
\hline $\begin{array}{c}\text { Integrated decision-making } \\
\text { over } \\
\text { Operation-independent }\end{array}$ & 0.0001 & 0.0006 & 0.0025 & 0.0060 \\
\hline $\begin{array}{c}\text { Integrated decision-making } \\
\text { over } \\
\text { Operation-dependent }\end{array}$ & 0.0077 & 0.0362 & 0.1865 & 0.1620 \\
\hline
\end{tabular}




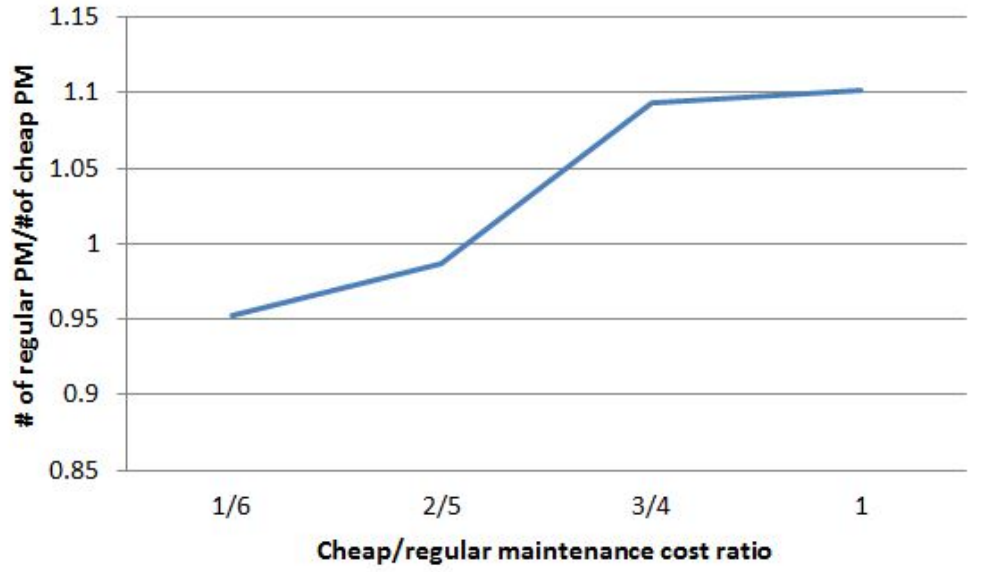

Figure 22. Comparison of number of regular and cheap PM events for various cost ratios

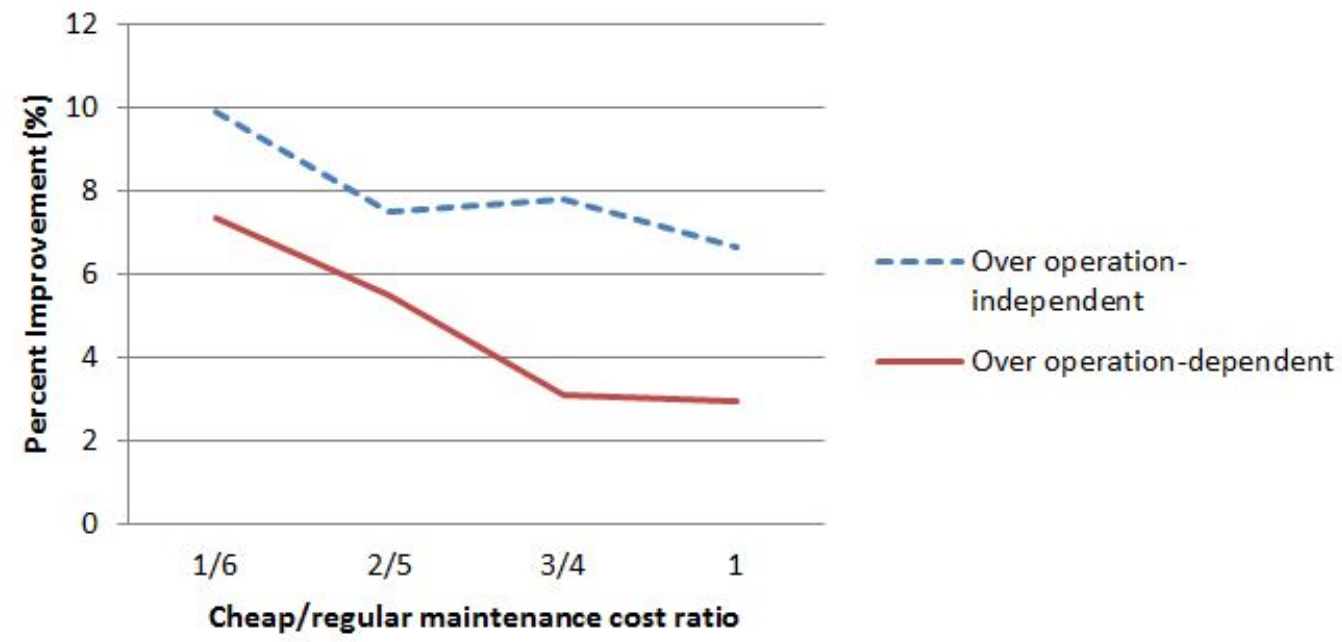

Figure 23. Percent improvement obtained by the integrated decision-making policy over the benchmark policies for different cheap/regular maintenance cost ratios 


\subsubsection{Influence of unmet production penalty costs $\left(p_{w_{j}}\right)$}

In this section, we investigate the performance of the newly proposed integrated decision-making policy under different unmet production penalty costs. The expected profit and percent improvements over the benchmark policies for various penalty costs are respectively given in Figure 24 and Figure 25. Once again, it is visible from Figure 24 that the expected profits obtained using the integrated decision-making policy are higher than the profits obtained by the benchmark policies, regardless of the unmet production penalty costs. Also, a closer look at the figure shows that as the penalty costs of unmet production grow, the expected profits obtained using the integrated decision-making policy diminish slightly while the expected profits for the operation-independent and operation-dependent CBM policies diminish considerably. The reason for this situation is that the integrated decision-making policy manages to meet the production goal more reliably by involving a smaller number of maintenance events, compared to the benchmark policies. This reduction in the number of maintenance events is due to the ability of the newly proposed decision-making scheme to change the product sequence and to keep assigning less degradation-sensitive products to more degraded machines. This is evident in Figure 26, where we see the expected total number of PM events for various unmet production penalty costs. 


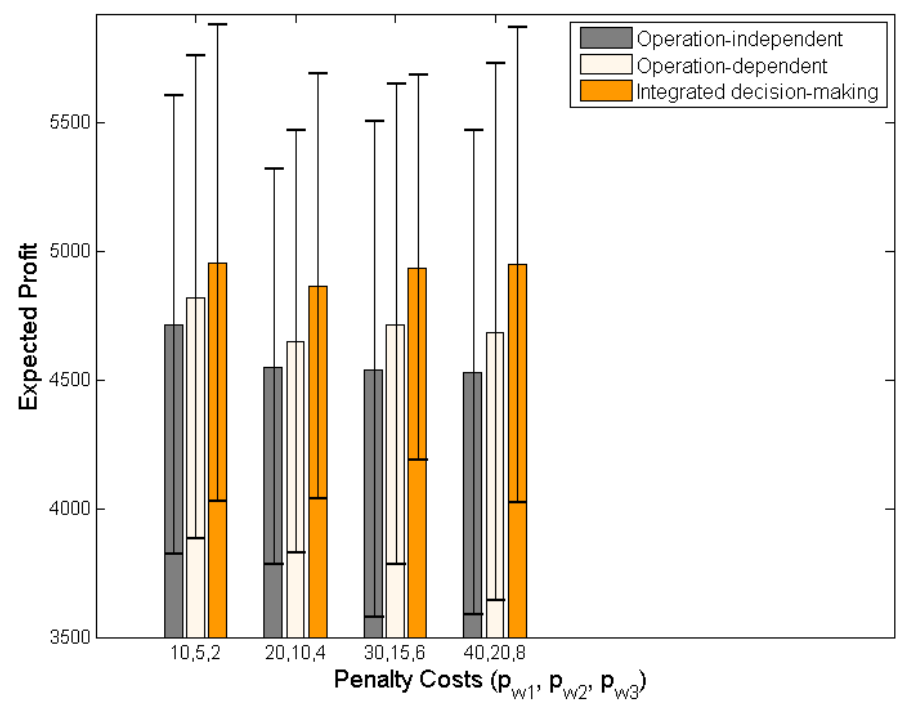

Figure 24. Comparison of expected profits for changing unmet production penalty costs (black lines denote the $-/+2 \sigma$ limits of the simulation outcomes) for the integrated decision-making policy, operation-dependent CBM policy and the traditional operationindependent CBM policy

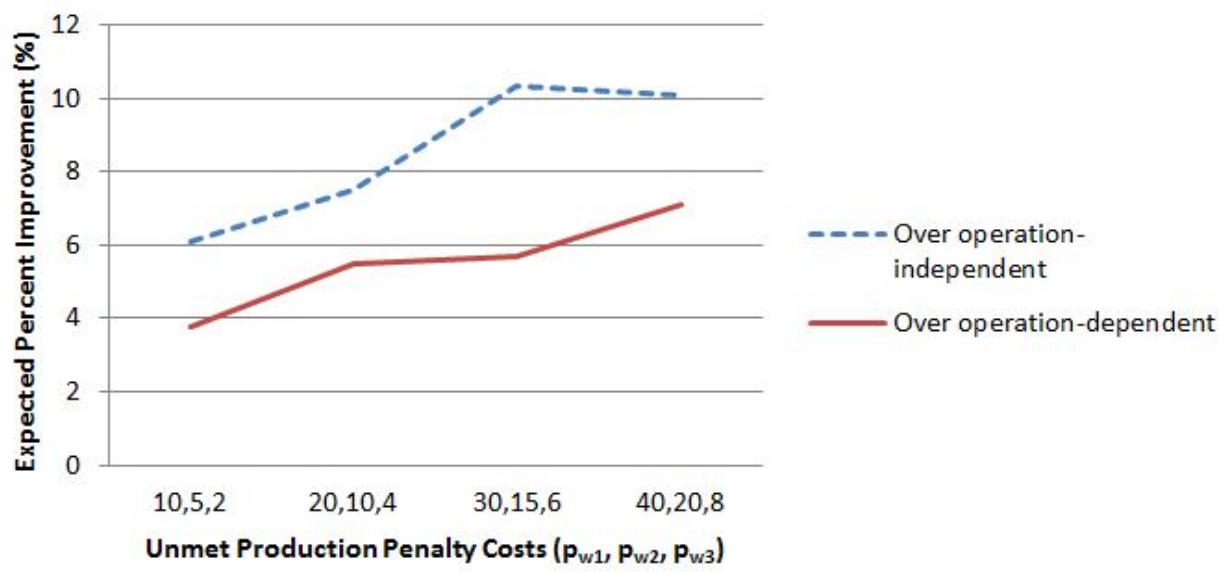

Figure 25. Percent improvement obtained by the integrated decision-making policy over the benchmark policies for different unmet production penalty costs 


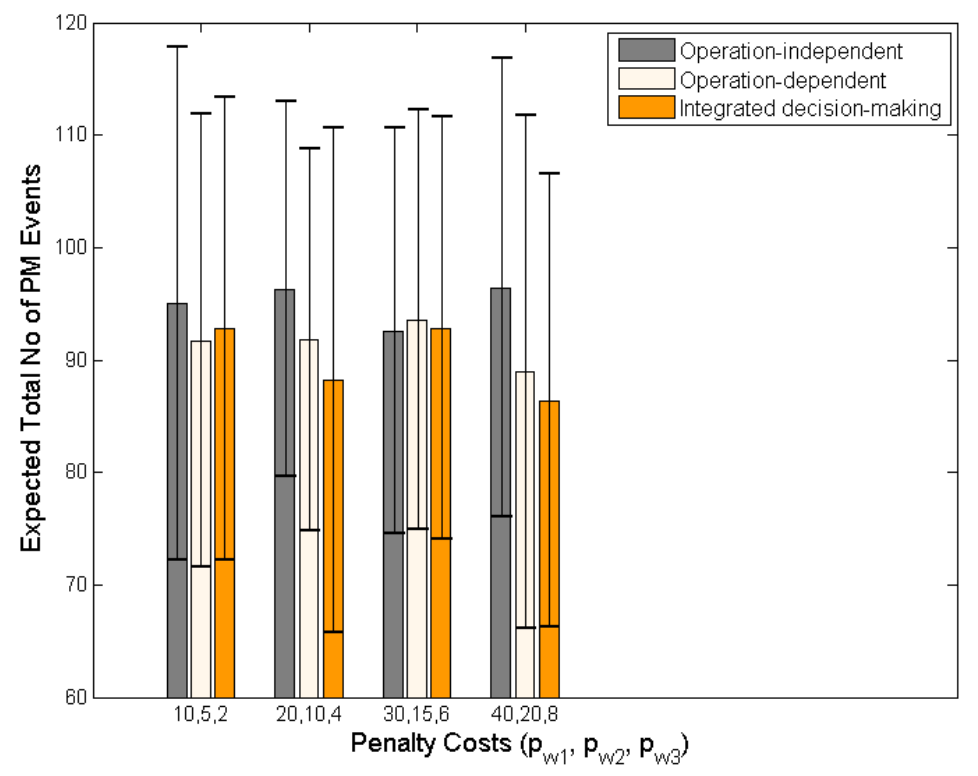

Figure 26. Expected total number of PM events for changing penalty costs (black lines denote $-/+2 \sigma$ limits of the simulation outcomes) for the integrated decision-making policy, operation-dependent CBM policy and the traditional operation-independent CBM policy

The same rationale also explains the results presented in Figure 25, which shows the percentual improvements brought by the newly proposed integrated decision-making scheme relative to the benchmark policies. The integrated decision-making policy is able to meet the production goal using fewer maintenance events, whereas the benchmark policies require more frequent maintenance interventions in order to meet that goal as the penalty costs increase. This explains why the relative benefits of the integrated decisionmaking policy over the benchmark policies consistently become larger as the unmet production penalty costs increase. 
8 shows the p-values calculated through a paired t-test. The proposed integrated decision-making policy significantly outperforms the operation-independent CBM policy in all instances. However, for the lowest penalty costs, the relative benefit of the integrated decision-making policy over operation-dependent CBM policy is the smallest and hence outperformance of the proposed method over operation-dependent CBM policy is not statistically significant.

Table 8. p-values for different penalty costs $\left(p_{w_{1}}, p_{w_{2}}, p_{w_{3}}\right)$

\begin{tabular}{|c|c|c|c|c|}
\cline { 2 - 5 } \multicolumn{1}{c|}{} & $10,5,2$ & $20,10,4$ & $30,15,6$ & $40,20,8$ \\
\hline $\begin{array}{c}\text { Integrated decision-making } \\
\text { over } \\
\text { Operation-independent }\end{array}$ & 0.0262 & 0.0006 & 0.0008 & 0.0001 \\
\hline $\begin{array}{c}\text { Integrated decision-making } \\
\text { over }\end{array}$ & 0.2036 & 0.0362 & 0.0301 & 0.0339 \\
\hline \begin{tabular}{c} 
Operation-dependent \\
\hline
\end{tabular} & & & \\
\hline
\end{tabular}

\subsubsection{Influence of production goals $\left(N_{w_{j}}\right)$}

Let us now assess the performance of the integrated decision-making methodology introduced in this chapter under increasingly demanding production goals ${ }^{9}$. The expected profits for the newly proposed and benchmark decision-making schemes are shown in Figure 27 and, once again, the integrated decision-making policy consistently yields higher expected profits, when compared to the benchmark policies.

\footnotetext{
${ }^{9}$ I.e. as one increases the production goals $N_{w_{j}}$ for each product type in the cost functions (3), (4) and (5).
} 
This can be explained by the fact that as the production goals increase, avoiding unscheduled downtime and production penalty costs by preventing the production of scrap wafers becomes increasingly influential on the effects of any decision-making policy. The integrated decision-making policy jointly optimizes product sequencing and operation-specific maintenance triggering states and thus offers more freedom in the operational decision-making. For example, for the operations that are executed in the completion of the wafers for which we have a higher production goal, the integrated decision-making policy can suggest earlier triggering of maintenance, thus reducing the number of scrap wafers and increasing the likelihood of reaching that production goal. It can also compensate for the consequent increase in the number of preventive maintenance events by suggesting later triggering of maintenance events for other operations. In addition, by changing the product sequence, the integrated decisionmaking has the flexibility to assign less sensitive operations to more degraded chambers, thus reducing the number of scrap wafers, and potentially postponing the maintenance of those machines towards periods of cheaper maintenance. The traditional operationindependent $\mathrm{CBM}$ policy does not have these flexibilities and as the production goals increase, in order to reduce the number of scrap wafers, the number of maintenance events, and hence maintenance costs, increase more dramatically than in the case of the integrated decision-making policy. This is clearly visible in Figure 29, which shows expected maintenance costs for the 3 policies considered in this study.

It is therefore logical that as the production goals increase, the relative benefits of the integrated decision-making policy over the traditional operation-independent CBM 
policy consistently increase, as can be seen in Figure 28. However, it can also be observed in Figure 28 that for the highest production goal, the percentual improvement of the integrated decision-making policy over the operation-dependent CBM policy actually decreases, as does the slope of the percentual improvements the newly proposed policy relative to the traditional CBM policy. The reason for this can be seen in Figure 30. Namely, even though the probability of meeting the production goal decreases for all maintenance policies as the production goal increases, the decrease in this probability for the integrated decision-making policy is more dramatic, as it prefers to decrease the number of maintenance events at the cost of the increased number of scrap wafers, which further reduces the probability of meeting the production goals. Therefore, despite the fact that the expected maintenance cost increases only slightly with the increasing production goals for the integrated decision-making policy, the decrease in the probability of meeting the production goal causes the pattern seen in Figure 28 for the most aggressive production goals. In Table 9, it is observed that the integrated decisionmaking policy outperforms the benchmark policies significantly despite the pattern observed in Figure 28. 


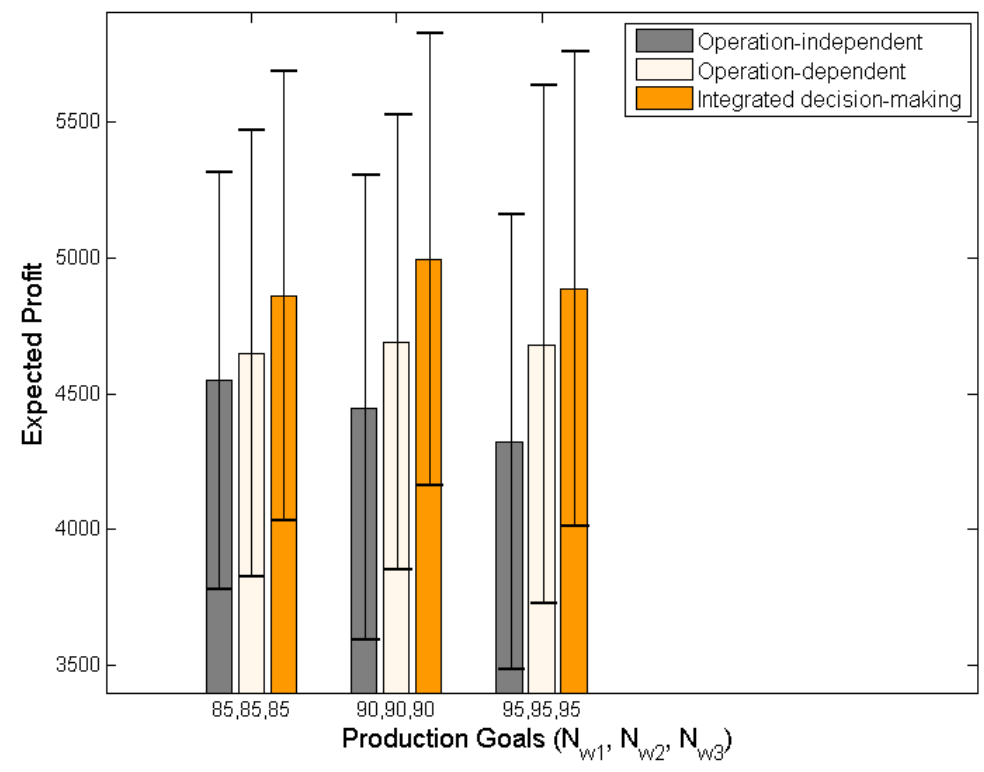

Figure 27. Comparison of expected profits for different production goals (black lines denote the $-/+2 \sigma$ limits of the simulation outcomes) for the integrated decision-making policy, operation-dependent CBM policy and the traditional operation-independent CBM policy

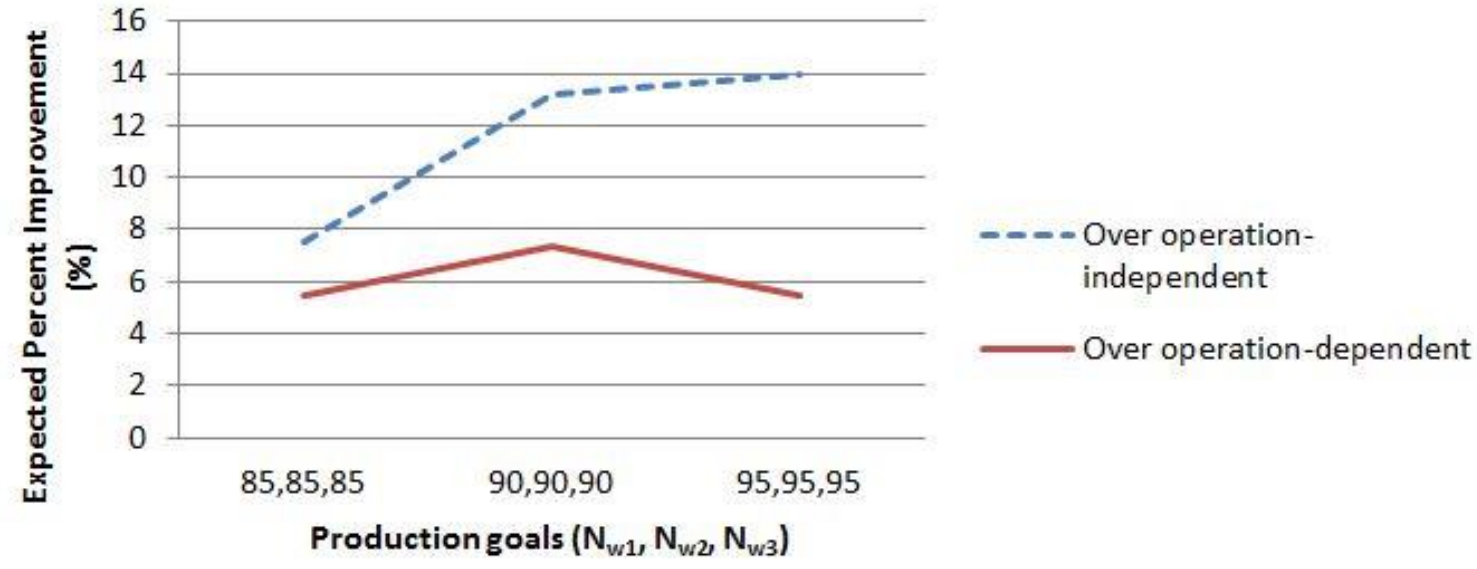

Figure 28. Percent improvement over benchmark policies obtained by the integrated decision-making policy for different production goals 


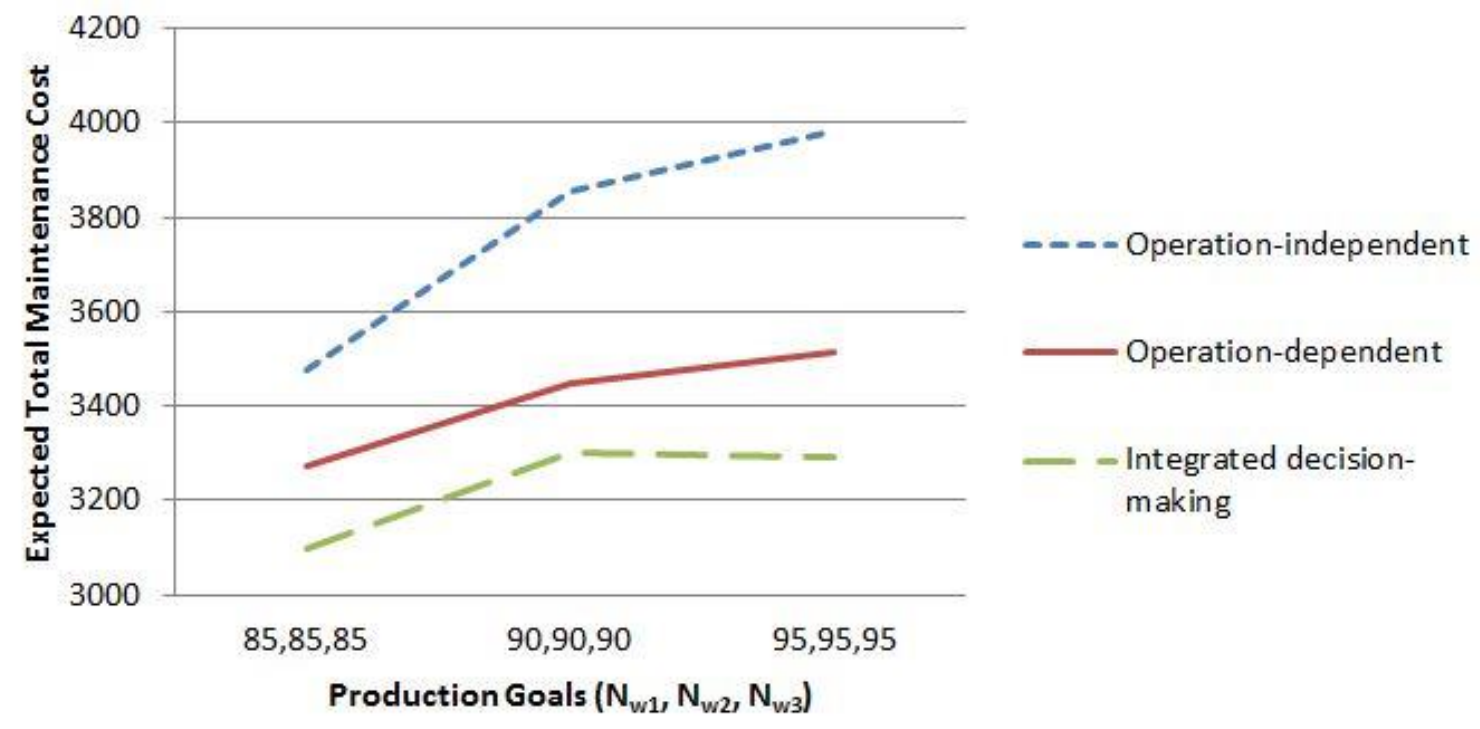

Figure 29. Total expected maintenance cost obtained by different maintenance policies for increasing production goals

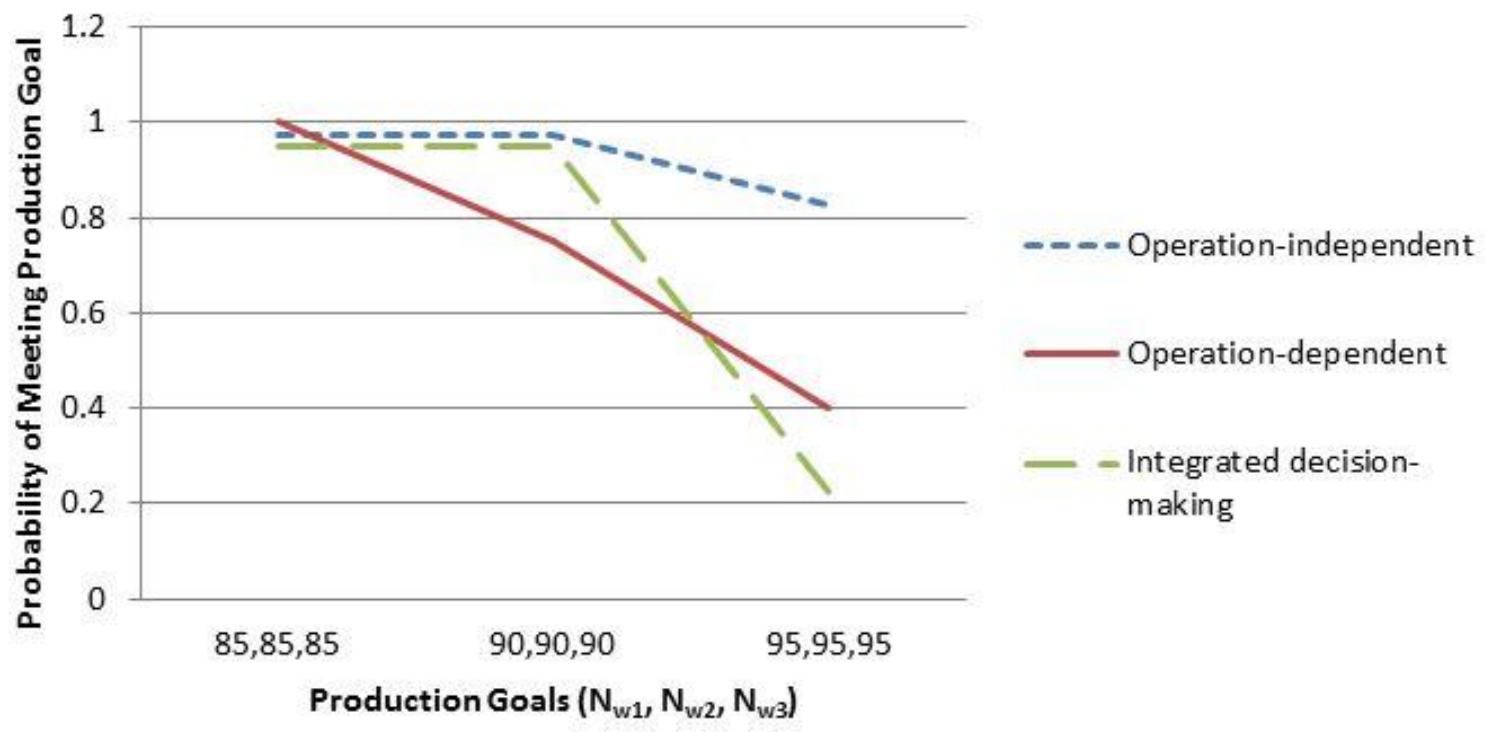

Figure 30. Probabilities of meeting the production goal for 3 decision-making policies over increasing production goals 
In order to observe the behavior of the integrated decision-making system under conditions where it is no longer more cost effective to sacrifice production for the sake of decreased maintenance cost, we increased the unmet production goal penalties and set them to be double the reward for each wafer. For these penalty parameters, we conducted another comparative study for the same production goals as those considered in Figure 28. Results of this study are illustrated in Figure 31, which shows percentual improvements of the integrated decision-making policy over the benchmark policies for the increased unmet production penalty costs. For these parameters, the cost of unmet production is so much more severe that the integrated decision-making policy no longer prefers sacrificing production in order to reduce maintenance costs, which is why results in Figure 31 shows consistently increasing percentual improvements over the benchmark policies.

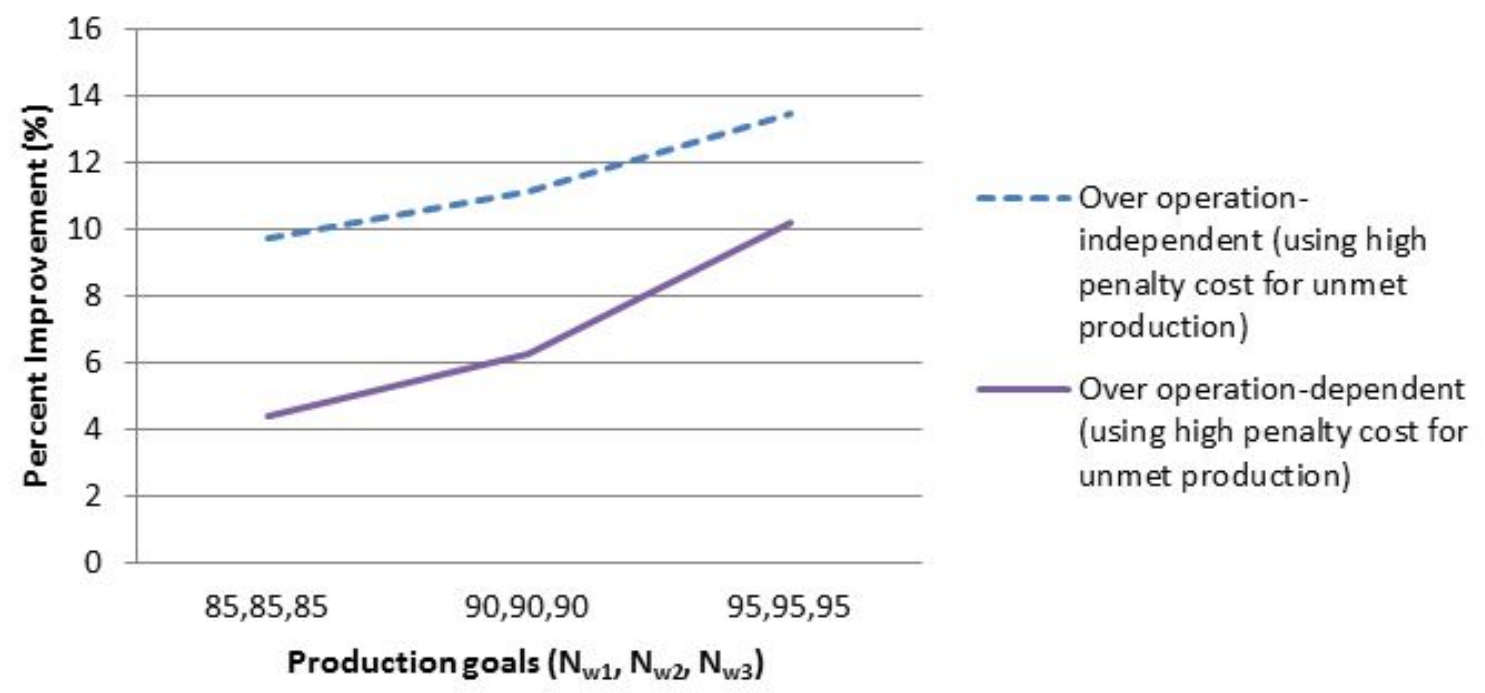

Figure 31. Percent improvement over the benchmark policies obtained by the integrated decision-making policy for higher unmet production penalty costs 
The p-values associated with higher penalty costs are presented in Table 10. As can be observed in Table 10, the proposed method significantly outperforms the benchmark policies in all but one instance. For the lowest production goal, the integrated decision-making policy yields the lowest relative benefits over the operation-dependent CBM policy and hence the outperformance is not statistically significant.

Table 9. p-values for different production goals $\left(N_{w_{1}}, N_{w_{2}}, N_{w_{3}}\right)$

\begin{tabular}{|c|c|c|c|}
\cline { 2 - 4 } \multicolumn{1}{c|}{} & $85,85,85$ & $90,90,90$ & $95,95,95$ \\
\hline $\begin{array}{c}\text { Integrated decision-making } \\
\text { over } \\
\text { Operation-independent }\end{array}$ & 0.0006 & 0.0001 & 0.0001 \\
\hline $\begin{array}{c}\text { Integrated decision-making } \\
\text { over }\end{array}$ & 0.0362 & 0.0017 & 0.0462 \\
\hline \begin{tabular}{c} 
Operation-dependent \\
\hline
\end{tabular} & & & \\
\hline
\end{tabular}

Table 10. p-values for different production goals $\left(N_{w_{1}}, N_{w_{2}}, N_{w_{3}}\right)$ with higher penalty costs

\begin{tabular}{|c|c|c|c|}
\cline { 2 - 4 } \multicolumn{1}{c|}{} & $85,85,85$ & $90,90,90$ & $95,95,95$ \\
\hline $\begin{array}{c}\text { Integrated decision-making } \\
\text { over }\end{array}$ & 0.0001 & 0.0001 & 0.0001 \\
\hline $\begin{array}{c}\text { Operation-independent } \\
\text { Integrated decision-making } \\
\text { over }\end{array}$ & 0.1228 & 0.0043 & 0.0003 \\
\hline Operation-dependent & & \\
\hline
\end{tabular}




\subsubsection{Influence of probabilities that preventive maintenance actions restore the perfect condition of the maintained system (MP)}

In this study, we acknowledge the fact that the effects of a maintenance operation may be uncertain. As mentioned in the problem description in Section 4.2, we acknowledge that imperfect maintenance may happen and will study in this section the effects of the effectiveness of maintenance operations on the expected profits yielded by different decision-making policies.

Various effectiveness levels of PM interventions are tested using different Markov transition matrices corresponding to the condition-state recoveries induced by PM events. Figure 32 lists the PM-related Markov transition matrices tested in this study. Generally speaking, these matrices express the effectiveness of maintenance operations via the expected equipment condition after a maintenance is executed. Matrices MP1 MP5 are selected in such a way that regardless of the state at which maintenance is invoked, as we go from MP1 to MP5, we see increasingly efficient PM interventions, with increasing probabilities that PM returns the maintained system to better states (closer to the "as good as new" state). Note that MP5 corresponds to perfect maintenance operations that always return the maintained system to the "as good as new" state. 


$$
\begin{aligned}
M P_{1}= & {\left[\begin{array}{ccccc}
1 & 0 & 0 & 0 & 0 \\
1 & 0 & 0 & 0 & 0 \\
0.20 & 0.80 & 0 & 0 & 0 \\
0.20 & 0.60 & 0.20 & 0 & 0 \\
1 & 0 & 0 & 0 & 0
\end{array}\right] M P_{2}=\left[\begin{array}{cccccc}
1 & 0 & 0 & 0 & 0 \\
1 & 0 & 0 & 0 & 0 \\
0.40 & 0.60 & 0 & 0 & 0 \\
0.40 & 0.45 & 0.15 & 0 & 0 \\
1 & 0 & 0 & 0 & 0
\end{array}\right] M P_{3}=\left[\begin{array}{ccccc}
1 & 0 & 0 & 0 & 0 \\
1 & 0 & 0 & 0 & 0 \\
0.60 & 0.40 & 0 & 0 & 0 \\
0.60 & 0.30 & 0.10 & 0 & 0 \\
1 & 0 & 0 & 0 & 0
\end{array}\right] M P_{4}=} \\
& {\left[\begin{array}{ccccc}
1 & 0 & 0 & 0 & 0 \\
1 & 0 & 0 & 0 & 0 \\
0.80 & 0.20 & 0 & 0 & 0 \\
0.80 & 0.15 & 0.05 & 0 & 0 \\
1 & 0 & 0 & 0 & 0
\end{array}\right] M P_{5}=\left[\begin{array}{lllll}
1 & 0 & 0 & 0 & 0 \\
1 & 0 & 0 & 0 & 0 \\
1 & 0 & 0 & 0 & 0 \\
1 & 0 & 0 & 0 & 0 \\
1 & 0 & 0 & 0 & 0
\end{array}\right] }
\end{aligned}
$$

Figure 32. Perfect maintenance probabilities used for sensitivity analysis with integrated decision-making policy

The expected profits obtained for various levels of maintenance efficacies are shown in Figure 33, while the percent improvements obtained with the integrated decision-making policy over the benchmark policies are given in Figure 34.

From Figure 33, we can once again observe that for all levels of PM efficacy, the integrated decision-making policy results in a higher expected profit, when compared to the benchmark policies. In addition, from Figure 34 one can see that higher levels of PM efficacy ${ }^{10}$ result in more pronounced percentual improvements of the newly proposed policy over the traditional operation-independent CBM policy. This can be explained by the fact that in sophisticated decision mechanisms, such as the newly proposed integrated decision-making, the success of decisions strongly depends on the effectiveness of the execution of those decisions. If the decisions are not executed effectively (when probabilities of restoring the good conditions of the maintained machine are lower), that

${ }^{10}$ Situations when Markovian transition matrices corresponding to PMs have higher likelihood of returning the system closer to the "as good as new" state, i.e. scenarios obtained as we go from Markovian transition matrix $\mathrm{MP}_{1}$, to $\mathrm{MP}_{2}$, then on to $\mathrm{MP}_{3}$ and so on. 
diminishes the benefits of the integrated decision-making policy and reduces the resulting profits.

In addition, it can also be observed from Figure 34 that as the maintenance efficacy increases, the percentual improvements obtained by the integrated decisionmaking policy over the operation-dependent CBM policy first rise and then decrease. This phenomenon can be explained as follows. When the maintenance efficacy is lower, the maintenance triggering threshold decisions in both operation-dependent CBM policy and the integrated decision-making policy are not reliable as the execution of these decisions is far from perfect and hence the benefits of the ability to rearrange the product sequencing become more pronounced. However, as the maintenance efficacy keeps increasing, the maintenance decisions are applied more and more effectively and hence the relative advantage of the integrated decision-making policy due to the ability to rearrange the product sequence decreases, resulting in the trend we see in Figure 34.

The p-values calculated through a paired t-test are presented in Table 11. As mentioned in the previous paragraph, when the maintenance efficacy is lower, both the integrated decision-making policy and the operation-dependent CBM policy fail to produce reliable maintenance triggering threshold decisions. Therefore, the proposed integrated decision-making policy does not produce significantly better results than the operation-dependent CBM policy for lower maintenance efficacies. 


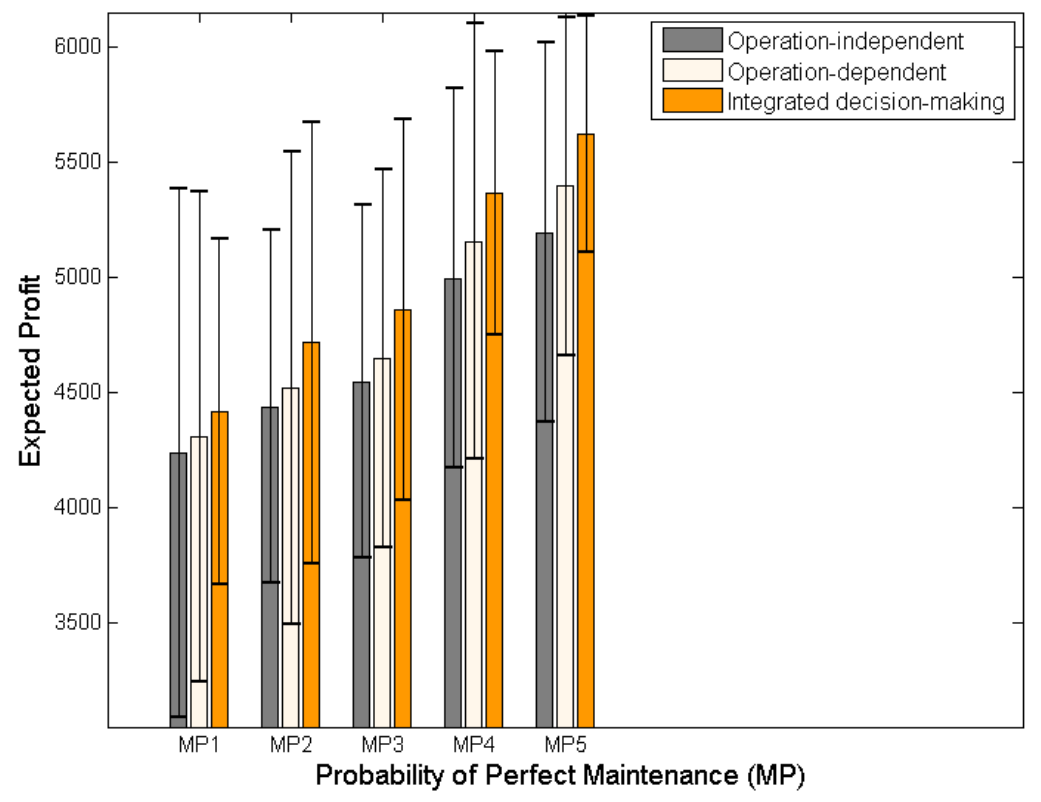

Figure 33. Comparison of expected profits for different probabilities of perfect maintenance (black lines denote the $-/+2 \sigma$ limits of the simulation outcomes) for the integrated decision-making policy, operation-dependent CBM policy and the traditional operation-independent CBM policy

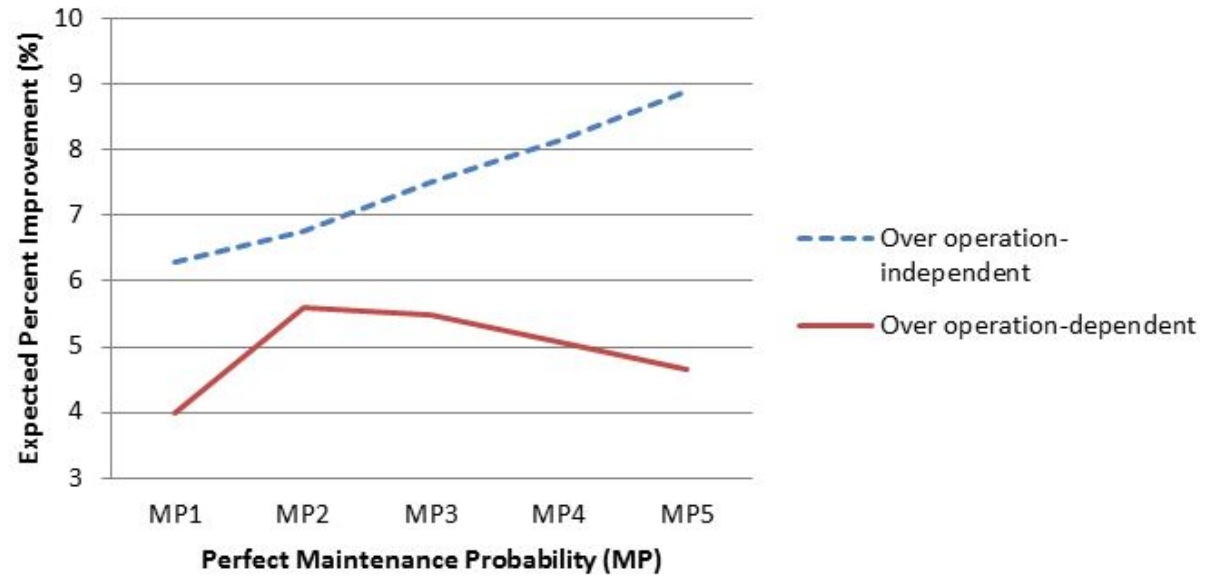

Figure 34. Percent improvement obtained by the integrated decision-making policy over the benchmark policies for different probabilities of perfect maintenance 
Table 11. p-values for different probabilities of perfect maintenance

\begin{tabular}{|c|c|c|c|c|c|}
\cline { 2 - 5 } \multicolumn{1}{c|}{} & MP1 & MP2 & MP3 & MP4 & MP5 \\
\hline $\begin{array}{c}\text { Integrated decision-making } \\
\text { over }\end{array}$ & 0.0978 & 0.0015 & 0.0006 & 0.0001 & 0.0001 \\
\hline $\begin{array}{c}\text { Operation-independent } \\
\begin{array}{c}\text { Integrated decision-making } \\
\text { over }\end{array} \\
\text { Operation-dependent }\end{array}$ & 0.2737 & 0.0828 & 0.0362 & 0.0255 & 0.0023 \\
\hline
\end{tabular}

\subsection{Conclusions}

In this chapter, a decision-making system for maintenance scheduling and product sequencing for a multiple machine/multiple operation manufacturing system is presented. To the best of our knowledge, this is the first integrated decision-making study in a manufacturing system consisting of multiple manufacturing stations that considers both operation-dependent degradation models and a model of the probability of manufacturing success yield, that is both operation and degradation dependent.

The newly introduced integrated decision making policy was applied on an example of a cluster tool model, which is a mini flexible manufacturing system. It was benchmarked against the traditional operation-independent condition based maintenance policy, as well as the operation-dependent condition based maintenance policy presented in Chapter 3. In addition, a sensitivity analysis was conducted to evaluate the performance of the newly proposed methodology under varying relative costs of maintenance in periods of cheap and regular maintenance interventions, penalties of unmet production, production goals and efficacy of maintenance. The results show that the integrated decision-making policy consistently outperforms the benchmark decision 
policies by providing a higher expected profit. Furthermore, it was observed that decreasing the ratio of costs of maintenance interventions during periods of cheap maintenance relative to those done during regular maintenance periods, as well as increasing of the unmet production penalty costs for unmet production, production goals and efficacy of maintenance execution all resulted in more significant benefits of the newly proposed integrated decision-making policy over the traditional operationindependent CBM policy. In addition, decreases in the cheap/regular maintenance cost ratios and increases in the penalties of unmet production consistently yielded increased relative benefits of the newly introduced policy over the operation-dependent CBM policy from Chapter 3. Finally, sensitivity studies regarding different production goals and efficacies of maintenance operations illustrated how an interplay of contributions from a reduction in the number of PM events, probabilities of meeting various production goals, penalties due to unmet production and reliability with which maintenance decision are executed lead to different trends in the relative improvements of the integrated maintenance and production sequencing policy over the operation-dependent CBM policy. 


\section{Chapter 5}

\section{Integrated Maintenance and Operations Decision Making with Imperfect Degradation State Observations}

\subsection{Introduction}

In Chapters 3 and 4, it was assumed that the degradation process of the underlying equipment condition follows a specific Markov Model with current conditions of the manufacturing environment being perfectly observable (states of the Markov Model were perfectly observable at any given time). However, in some highly complex manufacturing environments, such as highly integrated systems of multiple interacting subsystems, or in systems involving numerous distributed multi-physics phenomena, such as plasma-based tools in semiconductor manufacturing, the condition of the equipment cannot be directly observed and must be probabilistically inferred from the available sensor readings [142], [143]. Acknowledging this, recently it has been proposed to model degradation in such systems as a Hidden Markov Model (HMM), where degradation states of the monitored system are modeled as the hidden states of the HMM and the sensor readings are the observable symbols of the HMM. In such an environment, integrated decision-making of the operations and maintenance scheduling has not been addressed before and that is the gap that will be addressed in this chapter. 
In this chapter, we consider a FMS where the degradation processes of each machine are still characterized by operation-dependent Markov chains. However, the current degradation states of the machines are not perfectly observable and can only be inferred probabilistically from available sensor readings. The decisions of actions (execute PM, RM or operation) are made based on uncertain information. Therefore, this process is a Partially Observable Markov Decision Process (POMDP). A POMDP is a generalization of a Markov Decision Process (MDP). The system dynamics of a POMDP are determined by a MDP and there are still a set of states, a set of actions, state transitions and rewards for actions. However, contrary to a MDP, in POMDP, the current state of the system is not perfectly observable which adds a set of observations to the model.

The rest of this chapter is organized as follows. In Section 5.2, we give a brief overview of the latest progress in the application of POMDPs in the integrated decisionmaking realm. Section 5.3 will formally define the problem of integrated decisionmaking for maintenance scheduling and production sequencing in an FMS with imperfect degradation state observations, while Section 5.4 introduces a novel solution procedure for this joint decision-making problem. In order to assess the performance of the newly proposed decision-making methodology and compare it with some more traditional decision-making policies, the results collected from a series of numerical experiments based on the simulations of a semiconductor cluster tool are enclosed in Section 5.5. Finally, Section 5.6 discusses the conclusions of this work and possible directions for future research. 


\subsection{Overview of POMDP in Integrated Decision-Making}

Howard [144] described movement in an MDP as a frog in a pond jumping from lily pad to lily pad. On the other hand, the setting of a POMDP can be viewed as a fog shrouded lily pond. The frog is no longer certain about which pad it is currently on and before jumping, the frog can obtain information about its current location. POMDP's are suitable for manufacturing applications, where thorough inspection of the machine condition is costly and often infeasible. However, this suitability comes at the cost of added computational complexity.

POMDP models have been applied to areas like cost control problems in accounting [145], internal control of a corporate control system [146], learning process [147], job search [148], etc. However, we will only elaborate on the literature on the POMDP based maintenance decision-making for a deteriorating system.

The first works on the modeling of machine deterioration and maintenance as a POMDP mainly considered only two actual states for the current degradation of the machine (core states) and three possible actions (do nothing, do inspection, do maintenance). Girshick and Rubin [149] were one of the first to consider such a setting. They determined the forms of optimal maintenance policies in the case where no information regarding the condition of the station is obtained if "do nothing" action is selected and perfect information is obtained if "do inspection" option is selected. However, Taylor [150] showed the maintenance policy from [149] to be wrong via a counter-example. For the two-state core process, Ross [151] showed that the optimal 
policy may have four regions (produce without inspection - inspection - produce without inspection - repair), despite the common intuition of three regions (produce without inspection - inspection - repair). Recently, Ivy and Nembhard [152] proposed a simulation methodology for integrating statistical quality control and POMDP in order to develop and evaluate optimal maintenance policies for a two-state system.

Smallwood and Sondik [153] considered a more general setting with more than two core states. They showed that the optimal payoff function is piecewise linear and convex, and presented an algorithm using this property to calculate the optimal control policy over a finite horizon. White [154] has shown that only control limit strategies should be examined for finding an optimal policy for a partially observable replacement problem. Lovejoy [155] provided sufficient conditions for the optimal policy to be monotone on the space of state probability vectors ordered by likelihood ratios (totally positive of order 2, TP2). Maillart [57] considered a system with obvious failures and modeled both the perfect-information (observations perfectly reveal the underlying deterioration state) and imperfect-information (observations are only probabilistically related to the underlying deterioration state) cases as cost-rate-minimizing POMDP's. Elwany, Gebraeel and Maillart [156] focused on the exponentially increasing degradation models and showed that the optimal replacement policy for such models is a monotonically non-decreasing control limit policy.

In most manufacturing environments, especially in flexible manufacturing systems, a machine can execute multiple operations and hence its degradation model 
changes as the operation executed on it changes. However, few researchers have considered the influence of a varying environment on the maintenance decision-making. Byon, Ntaimo and Ding [157] examined optimal repair strategies that minimizes the expected average cost over an infinite horizon for wind turbines operating under stochastic weather conditions. The closest work in literature to the proposed work in this chapter was presented by Jin, Miyawaki and Suzuki [158]. They considered a system that deteriorates under the influence of a controllable varying environment. They identified a sufficient condition that ensures the optimality of the control limit policy with respect to the system's prior probability and its environment. In [158], the degradation of the machine is affected by the varying environment, but the condition of the environment is not affected by the degradation of the machine.

In our work, the degradation of the machines influences our decisions on the dispatching of the operations to the machines, which effectively means decision-making will affect the operating regime of the machine ("environment" variable from [158]). Furthermore, in all of the studies presented above, the cost of each action (production, preventive maintenance, reactive maintenance) is assumed to be known. In our study, the degradation state of a machine affects the probability of successful completion of an operation and too many unsuccessful operations may lead to an unmet production goal penalty. Hence, in the study proposed in this document, it is much more challenging to express the cost effects of actions, compared to what we see in [158]. The aforementioned challenges will be addressed in the remainder of this chapter. 


\subsection{Problem Statement}

In this chapter, we consider a similar problem to the one described Chapter 4, with a crucial modification in the sense that the degradation states of the manufacturing stations are not perfectly observable anymore and can only be inferred from the available sensor readings. More precisely, we consider a manufacturing environment with $m$ stations labeled $c_{1}, c_{2}, \ldots, c_{m}$, producing a set of product types $W=\left\{w_{1}, w_{2}, \ldots, w_{l}\right\}$ via a set $O=\left\{o_{1}, o_{2}, \ldots, o_{K}\right\}$ of operations that can be executed by the stations of that manufacturing system. It is assumed that each station $c_{i}$ can execute operations $O_{c_{i}}=$ $\left\{o_{c_{i}}^{1}, o_{c_{i}}^{2}, \ldots, o_{c_{i}}^{q_{i}}\right\} \subset O$, where $q_{i}$ is the number of operations that can be executed by that station. Any two stations $c_{i_{1}}$ and $c_{i_{2}}$ may be able to execute some common operations, which means that $O_{c_{i_{1}}} \cap O_{c_{i_{2}}}$, for $c_{i_{1}}, c_{i_{2}} \in\left\{c_{1}, c_{2}, \ldots, c_{m}\right\}$ may not necessarily be an empty set. Each product type $w_{j}, w_{j} \in W$, is associated with a sequence of operations $O_{w_{j}}=\left[o_{w_{j}}^{1}, o_{w_{j}}^{2}, \ldots, o_{w_{j}}^{p_{j}}\right]$, where $p_{j}$ is the number of operations needed to manufacture product type $w_{j}$. Successful completion of $w_{j}$ requires all the operations in the sequence $O_{w_{j}}$ to be executed successfully. The goal will be to produce $N_{w_{j}}$ of each product type $w_{j}, w_{j} \in W$, within a certain mission time $T$.

$X_{i}(t)$ denotes the unobservable degradation state of station $c_{i}$ at time $t$, where $X_{i}(t) \in S_{c_{i}}$ and $S_{c_{i}}=\left\{1,2, \ldots, M_{c_{i}}\right\}$. The initial distribution of $X_{i}(t)$ is given by $\pi_{i}(0)=$ $\left[\operatorname{Pr}\left(X_{i}(0)=1\right), \operatorname{Pr}\left(X_{i}(0)=2\right), \ldots, \operatorname{Pr}\left(X_{i}(0)=M_{c_{i}}\right)\right]$. The unobservable degradation 
states are assumed to evolve according to an operation-dependent Markov process and the station and operation-dependent transition probabilities are represented in matrix form

$$
\boldsymbol{P}_{c_{i}}^{\left(o_{k}\right)}=\left[\begin{array}{ccc}
p_{c_{i}, 1,1}^{\left(o_{k}\right)} & \cdots & p_{c_{i}, 1, M_{c_{i}}}^{\left(o_{o}\right)} \\
\vdots & \ddots & \vdots \\
p_{c_{i}, M_{c_{i}, 1}}^{\left(o_{k}\right)} & \cdots & p_{c_{i}, M_{c_{i}, M_{c_{i}}}}^{\left(o_{k}\right)}
\end{array}\right]
$$

where $p_{c_{i}, u, v}^{\left(o_{k}\right)}$ is the probability of transitioning from state $u$ to $v$ when operation $o_{k}$ is executed on station $c_{i}$. The transition probability matrix $\boldsymbol{P}_{c_{i}}^{\left(o_{k}\right)}$ will be an upper triangular matrix when a production operation is executed, whereas it will be a lower triangular matrix when a maintenance event is executed. Thus, we assume that when a maintenance operation is performed, the condition of a station will get better, but it may not necessarily go to the "good as new" state.

As mentioned before, the underlying degradations states $X_{i}(t)$ are not perfectly observable. Instead, we have observations that give probabilistic information related to the true state of the system. Let $b_{i}(t)$ denote the observation emitted by station $c_{i}$ at time $t$ and be defined over the state space $B_{c i}=\left\{\gamma_{1}, \gamma_{2}, \ldots, \gamma_{N_{c i}}\right\}$. Observations are emitted from each state according to the $M_{c_{i}} \times N_{c_{i}}$ dimensional emission probability matrix

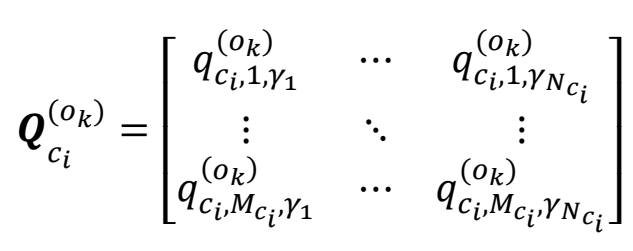


where $q_{c_{i}, j, \gamma_{k}}^{\left(o_{k}\right)}$ is the probability of observing symbol $\gamma_{k}$ when the actual degradation state of station $c_{i}$ is $j$ and action $o_{k}$ is executed on it. In this work, emission probability matrix $\boldsymbol{Q}_{c_{i}}^{\left(o_{k}\right)}$ is assumed to be known.

In addition, let us take into account the fact that as the degradation level of a station increases, the probability of success of any operation executed on that station decreases. It will be assumed that for any station, the probability of success of a given operation is a known function of the operation $o_{k} \in O$ and the station state $X_{i}$, denoted as $s_{c_{i}}\left(o_{k}, X_{i}\right)$.

Let $\left[d_{1}, \ldots, d_{F}\right]$ denote the production sequence in which the products are processed in the FMS, where $d_{q} \in\left\{w_{1}, w_{2}, \ldots, w_{l}\right\}, q=1,2, \ldots, F$ and $F$ is the total amount of products that are fed into the manufacturing system ${ }^{11}$. In this chapter, similar to Chapter 4 , it will be assumed that the FMS is such that the sequence $\left[d_{1}, \ldots, d_{F}\right]$ in which products are released into the FMS can also be changed. This means that the goal of producing a given number of units of each product type produced in a system could be achieved through multiple different sequences in which the units are released into it.

Based on the product sequence and already completed operations for a product, next operation to be executed is determined, i.e. $o_{k}$ where $o_{k} \in\left\{o_{1}, o_{2}, \ldots, o_{K}\right\}$. If operation $o_{k}$ can be executed more than one station, following the reasoning from [3], and job dispatching policy pursued in [7], [8], it will be dispatched to the station with the

\footnotetext{
${ }^{11}$ Effectively, $\left[d_{1}, \ldots, d_{F}\right]$ is the sequence in which the products are released into the system.
} 
lowest expected degradation state ${ }^{12}$, where the expected state of station $c_{i}$ at time $t$ is evaluated as

$$
\sum_{u=1}^{M_{c_{i}}} u \pi_{i u}(t)
$$

The action $a_{t+1}$ to be taken at time $t+1$, will be determined based on the information obtained from the distribution $\pi_{i}(t+1)$ of hidden states of station $c_{i}$ at time $t+1$. It will be assumed that a failed state is perfectly observable and if a station $c_{i}$ enters that failed state, it immediately shutdowns, requiring a reactive maintenance action to bring it back up. If the station is not in failed state, but the available observations indicate a situation in which probability that the degradation progressed beyond the maintenance triggering threshold for that station is sufficiently big, a preventive maintenance event will be triggered. More precisely, for a station $c_{i}$ on which operation $o_{k}$ needs to be performed at time $t+1$, if

$$
\sum_{v \geq S_{c_{i}}^{o_{k}}} \pi_{i v}(t+1) \geq \alpha
$$

where $\alpha$ is a tunable parameter ${ }^{13}$, operation $o_{k}$ could not be processed on it unless PM event is done on the station.

The decision-making objective will be to find a combined policy of maintenance triggering states across a system and product-type sequencing which maximizes a reward

\footnotetext{
12 This is a modification from what was done in [7], [8], because degradation states are now hidden.

${ }^{13} \alpha$ is one of the parameters for which a sensitivity analysis will be conducted.
} 
function that considers the benefits of production, costs of maintenance and penalties for unmet production goals. More formally, the decision optimization problem will be defined as follows. Let $N_{w_{j}}$ denote the production goal for product type $w_{j} \in$ $\left\{w_{1}, w_{2}, \ldots, w_{l}\right\}$, and let $n_{w_{j}}$ denote the number of good products of type $w_{j}$. The integrated decision-making policy will be pursued through the optimization procedure

$$
\begin{aligned}
& \underset{\left[d_{1}, d_{2}, \ldots, d_{F}\right]}{\operatorname{maximize}} E\left(\sum_{j=1}^{l} R_{w_{j}} n_{w_{j}}-V_{r} c_{r}-V_{p} c_{p}-V_{c r} c_{c r}-V_{c p} c_{c p}-\sum_{j=1}^{l} a_{w_{j}}\left(N_{w_{j}}-n_{w_{j}}\right) p_{w_{j}}\right) \\
& {\left[\begin{array}{cccc}
{\left[d_{1}, d_{2}, \ldots, d_{F}\right]} & \\
S_{c_{1}}^{o_{1}} & S_{c_{2}}^{o_{1}} & \cdots & S_{c_{m}}^{o_{1}} \\
S_{c_{1}}^{o_{2}} & S_{c_{2}}^{o_{2}} & \cdots & S_{c_{m}}^{o_{2}} \\
\vdots & \vdots & \ddots & \vdots \\
S_{c_{1}}^{o_{K}} & S_{c_{2}}^{o_{K}} & \cdots & S_{c_{m}}^{o_{K}}
\end{array}\right]}
\end{aligned}
$$

where

- $\quad R_{w_{j}}$ is the unit reward for good products produced of type $w_{j}, w_{j} \in W$

- $\quad p_{w_{j}}$ is the unit penalty for penalizing unmet production for product type $w_{j}, w_{j} \in W$

- $\quad c_{r}$ denotes the unit cost of reactive maintenance $(\mathrm{RM})$ per reactive maintenance event

- $c_{p}$ is the unit cost of preventive maintenance $(\mathrm{PM})$ per preventive maintenance event

- $c_{c r}$ denotes the unit cost of RM conducted during time intervals in which maintenance operation can be performed at a cheaper price (such as night shifts, weekends $)^{14}$

- $c_{c p}$ is the unit cost of PM executed during time intervals in which maintenance operations can be executed at a cheaper price ${ }^{15}$

\footnotetext{
${ }^{14}$ Of course, if such periods of cheaper maintenance do not exist in the given FMS, then $c_{c r}=c_{r}$.

${ }^{15}$ Once again, if such periods of time do not exist in a given FMS, then $c_{c p}=c_{p}$.
} 
- $V_{r}$ is the total number of RM events

- $V_{p}$ is the total number of PM events

- $V_{c r}$ is the total number of RM events during less expensive maintenance time periods

- $\quad V_{c p}$ is the total number of PM events during less expensive maintenance time periods

- $a_{w_{j}}=\left\{\begin{array}{c}1, \text { if } n_{w_{j}}<N_{w_{j}} \\ 0, \text { otherwise }\end{array}\right.$ for $\forall w_{j} \in W$

The objective function shown in (1) aims to find the product sequence $\left[d_{1}, d_{2}, \ldots, d_{F}\right]$ and operation-dependent maintenance triggering states $S_{c_{i}}^{o_{k}}$ that maximize the expected reward function in (1).

We understand that cost models in different companies, or even within different parts of the same company could be very different. Therefore, we pursued a method that is not dependent on the cost function in the sense that we used simulation based metaheuristic optimization where the cost function can easily be changed to suit the reality of a company, while the optimization method would not change at all. In order to demonstrate our method, we utilized a very simple and intuitive objective function (1) that rewards production and punishes downtime and scrap products. Interested readers/users can easily change the objective function to accommodate the needs of their production systems.

The expectation operator in (1) is applied because of the stochastic effects induced by randomness in the reliability of equipment (states of manufacturing stations 
degrade according to operation specific and machine specific stochastic processes), stochastic relations between equipment condition and available observations, conditiondependent yields (for each operation and each machine, one has a probability of successfully completing the operation), maintenance effects (equipment condition after maintenance is stochastic) and repair times (repair times are not modeled deterministically). All these random effects are modeled via discrete event simulations and objective function results obtained from multiple runs of the simulations are averaged to estimate the expected cost effects corresponding to any given product sequence and maintenance triggering policy.

In order to assess the performance of the newly proposed methodology, the integrated decision-making policy introduced in this chapter will be compared with the traditional Operation-Dependent CBM Policy and Operation-Independent CBM Policy. Both benchmark policies will be modeled similarly to the newly proposed integrated decision-making policy such that in both benchmark policies the degradation is modeled by operation-dependent Markov chains. In both benchmark policies, the degradation states are not perfectly observable and can only be inferred from observed symbols.

The main difference of the operation-dependent CBM policy from the integrated decision-making policy is that the product type sequence $\left[d_{1}, d_{2}, \ldots, d_{F}\right]$ is assumed to be a priori given. The action selection process for operation-dependent CBM policy is the same as the action selection process for the integrated decision-making policy. If operation $o_{k}$ can be executed more than one station, it will be dispatched to the station 
with the lowest expected degradation state. The action $a_{t+1}$ to be taken at time $t+1$, will be determined based on the information obtained from the distribution $\pi_{i}(t+1)$ of hidden states of station $c_{i}$ at time $t+1$. If the station is in failed state, it is perfectly observable and requires a RM event. If the station is not in failed state, but the available observations indicate a situation in which probability that the degradation progressed beyond the maintenance triggering threshold for that station is sufficiently big, a preventive maintenance event will be triggered. Operation-dependent CBM policy will be pursued through the optimization procedure

$$
\begin{gathered}
\operatorname{maximize} \\
{\left[\begin{array}{cccc}
S_{c_{1}}^{o_{1}} & S_{c_{2}}^{o_{1}} & \cdots & S_{c_{m}}^{o_{1}} \\
S_{c_{1}}^{o_{2}} & S_{c_{2}}^{o_{2}} & \cdots & S_{c_{m}}^{o_{2}} \\
\vdots & \vdots & \ddots & \vdots \\
S_{c_{1}}^{o_{K}} & S_{c_{2}}^{o_{K}} & \cdots & S_{c_{m}}^{o_{K}}
\end{array}\right]}
\end{gathered} \quad E\left(\sum_{j=1}^{l} R_{w_{j}} n_{w_{j}}-V_{r} c_{r}-V_{p} c_{p}-V_{c r} c_{c r}-V_{c p} c_{c p}-\sum_{j=1}^{l} a_{w_{j}}\left(N_{w_{j}}-n_{w_{j}}\right) p_{w_{j}}\right)
$$

For the operation-independent CBM policy, the product type sequence $\left[d_{1}, d_{2}, \ldots, d_{F}\right]$ is again assumed to be a priori given. In this policy, the inferred maintenance triggering states for each station are independent of the operations executed on it (i.e. $S_{c_{i}}^{o_{1}}=S_{c_{i}}^{o_{2}}=\cdots=S_{c_{i}}^{o_{K}}=S_{c_{i}}$ for each station $c_{i}$ ). The action selection process for this policy is similar to the other benchmark policy and the proposed integrated decision-making policy. However, it differs in the way a PM event is triggered which is independent of the operation executed on that station for this policy. More precisely, a PM event is triggered if

$$
\sum_{v \geq S_{c_{i}}} \pi_{i v}(t+1) \geq \alpha .
$$


For completeness, the optimization procedure for operation-independent CBM policy is as follows:

$\underset{\left[\begin{array}{llll}S_{c_{1}} & S_{c_{2}} & \cdots & S_{c_{m}}\end{array}\right]}{\operatorname{maximize}} E\left(\sum_{j=1}^{l} R_{w_{j}} n_{w_{j}}-V_{r} c_{r}-V_{p} c_{p}-V_{c r} c_{c r}-V_{c p} c_{c p}-\sum_{j=1}^{l} a_{w_{j}}\left(N_{w_{j}}-n_{w_{j}}\right) p_{w_{j}}\right)$

It should be mentioned that, the integrated decision-making policy and the benchmark CBM policies described above are stationary in the sense that the selected maintenance triggering states and product type sequence would not change throughout the execution of all production.

\subsection{Solution Procedure}

\subsubsection{Degradation State Estimation}

Since the current degradation states of the stations are not perfectly observable, they can be only inferred from the observations. The estimations on the current state of a station at time $t+1$ are dependent on the action taken at time $t$ and the observable symbol emitted by the station at time $t+1$. To estimate condition of the station $c_{i}$ at time $t+1$, given that symbol $\gamma_{k}$ was observed at time $t+1$, the following commonly used Bayes' formula can be employed to obtain the probability density of hidden states at time $t+1[159]:$

$$
\pi_{i v}(t+1)=\frac{\sum_{u \in S_{c i}} q_{i v \gamma_{k}} p_{c_{i}, u, v}^{a_{t}} \pi_{i u}(t)}{\sum_{v \prime \in S_{c i}} \sum_{u \in S_{c i}} q_{i v \prime \gamma_{k}} P_{c_{i}, u, v,}^{a_{t}} \pi_{i u}(t)}, \quad v=1, \ldots, M_{c_{i}}
$$




\subsubsection{Solution Representation}

For an $m$-station manufacturing system with $K$ operations that can be executed in these stations, a solution for preventive maintenance triggering states can be represented with a $K \times m$ matrix illustrated in Figure 35(a). In this matrix, $i^{\text {th }}$ column represents the maintenance triggering states of station $c_{i}$ for each operation, and similarly, $k^{\text {th }}$ row represents the maintenance triggering states of each station when operation $o_{k}$ is executed in it. In addition, the product type sequence $\left[d_{1}, d_{2}, \ldots, d_{F}\right]$ can be represented as an array of symbols denoting the product types, as shown in Figure 35 (b). The solution space is too large for complete enumeration, especially when candidate solutions are evaluated via replicated discrete-event simulations. On the other hand, the objective function does not have tractability, i.e. the problem at hand is a big stochastic optimization with no clear structure to reduce its complexity ${ }^{16}$. Hence, in order to find a practical, sub-optimal solution, a Tabu Search metaheuristic algorithm [105] is used, with discrete-event simulations of the target manufacturing system providing cost effects of any candidate decision. The cost effects of a candidate solution are evaluated via averaging the objective function values obtained from multiple replications of the discrete-event simulations of the target manufacturing system, which is fed back into the Tabu Search algorithm to guide the movements towards improved candidate decisions.

\footnotetext{
${ }^{16}$ As mentioned earlier, we pursued flexibility of the cost function (i.e. the objective function), to deal with the reality that even different departments within the same company could have different needs to be represented in the cost function.
} 


\begin{tabular}{|c|c|c|c|}
\hline$S_{c_{1}}^{o_{1}}$ & $S_{c_{2}}^{o_{1}}$ & $\cdots$ & $S_{c_{m}}^{o_{1}}$ \\
\hline$S_{c_{1}}^{o_{2}}$ & $S_{c_{2}}^{o_{2}}$ & $\cdots$ & $S_{c_{m}}^{o_{2}}$ \\
\hline$\cdots$ & $\cdots$ & $S_{c_{i}}^{o_{k}}$ & $\cdots$ \\
\hline$S_{c_{1}}^{o_{K}}$ & $S_{c_{2}}^{o_{K}}$ & $\cdots$ & $S_{c_{m}}^{o_{K}}$ \\
\hline
\end{tabular}

Candidate solution matrix representation

\begin{tabular}{|c|}
\hline$S_{c_{i}}^{o_{1}}$ \\
\hline$S_{c_{i}}^{o_{2}}$ \\
\hline$\cdots$ \\
\hline$S_{c_{i}}^{o_{K}}$ \\
\hline
\end{tabular}

\begin{tabular}{|c|c|c|c|}
\hline$S_{c_{1}}^{o_{k}}$ & $S_{c_{2}}^{o_{k}}$ & $\cdots$ & $S_{c_{m}}^{o_{k}}$ \\
\hline
\end{tabular}

Maintenance policy for operation $o_{k}$

(a)

\begin{tabular}{|l|l|l|l|l|}
\hline$d_{1}$ & $d_{2}$ & $d_{3}$ & $\cdots$ & $d_{F}$ \\
\hline
\end{tabular}

(b)

Figure 35. Representation of a candidate solution for the integrated decision-making policy

\subsubsection{Tabu Search Algorithm}

Tabu Search has recently begun to receive attention in the realm of maintenance optimization problems ([160] - [109]). The main idea of Tabu Search is to mark a candidate solution as 'tabu' once it has been evaluated so that the same candidate solution is not revisited over a certain number of iterations. The search starts from a randomly seeded or problem specific initial solution and moves iteratively from a non-tabu candidate solution $x$ to another non-tabu solution $x^{\prime}$ in the local neighborhood of $x$.

In this chapter, similar to Chapter 4 , the local neighborhood of any candidate solution is created by generating neighborhoods for the candidate maintenance triggering states and product type sequence, similarly to how it was done in [8]. For the sake of 
completeness, let us repeat the description of how it was done. In order to create a neighbor for the maintenance triggering states of the current solution, a cell in the corresponding matrix (shown in Figure 35 (a)) is selected randomly and its value is perturbed. This procedure is repeated a predetermined number of times in order to generate a local neighborhood of the current solution. For the product type sequence, neighbors were generated via a predetermined number of pairwise interchanges of two randomly selected product types (two randomly selected cells in the array representing the product sequence in Figure 35 (b)).

It should be noted that in this work, the operation-dependent CBM policy presented in Section 5.3 was used as the initial solution in order to speed up the metaheuristic optimization process ${ }^{17}$.

A tabu list is implemented to prevent returning to the solutions already visited for a certain number of iterations. However, if the best solution among the candidate solutions is obtained by a tabu move that yields an expected profit higher than the best profit obtained thus far, then its tabu status is overridden and it was allowed to be selected as the next incumbent solution. In other words, in the Tabu Search employed in this chapter, we used the well documented aspiration criterion concept, often used in the Tabu Search literature [104].

\footnotetext{
${ }^{17}$ The initial product sequence was randomly chosen, though that portion of the initial candidate solution can also be chosen more intelligently, to further accelerate the search, if some structure of prior knowledge about the problem exists.
} 
The algorithm terminates whenever the maximum number of iterations is reached or no improvements are made for a certain number of consecutive iterations.

\subsubsection{Solution Procedure for Benchmark Policies}

\subsubsection{Solution Procedure for Operation-Dependent CBM Policy}

The solution procedure for operation-dependent CBM policy is very similar to the solution procedure for the integrated decision-making policy described in Sections 5.4.1 -5.4 .3 .

As mentioned before, in the benchmark operation-dependent CBM policy, the current degradation states are not perfectly observable. The probability density of hidden states of station $c_{i}$ at time $t+1$, given that action $a_{t}$ was taken at time $t$ and symbol $\gamma_{k}$ was observed at time $t+1$, can be estimated using the following formula:

$$
\pi_{i v}(t+1)=\frac{\sum_{u \in S_{c i}} q_{i v \gamma_{k}} p_{c_{i}, u, v}^{a_{t}} \pi_{i u}(t)}{\sum_{v \prime \in S_{c i}} \sum_{u \in S_{c i}} q_{i v \prime \gamma_{k}} P_{c_{i}, u, v \prime}^{a_{t}} \pi_{i u}(t)}, \quad v=1, \ldots, M_{c_{i}}
$$

Each solution can be represented as shown in Figure 2 and Figure 35 (a) and as explained in Section 5.4.2. For operation-dependent CBM policy, a Tabu Search metaheuristic algorithm is used, with discrete-event simulations of the target manufacturing system providing cost effects of any candidate solution. The local neighborhood of any candidate solution is created by generating neighborhoods for the candidate maintenance triggering states similarly to how it was done in [7]. The local neighborhood generation for maintenance triggering states has been explained in detail in 
Section 5.4.3 and will not be repeated here. However, it should be mentioned that for the operation-dependent $\mathrm{CBM}$ policy, unlike the integrated decision-making policy, a neighborhood for product type sequence will not be generated as the product type sequence $\left[d_{1}, d_{2}, \ldots, d_{F}\right]$ is assumed to be a priori given.

\subsubsection{Solution Procedure for Operation-Independent CBM Policy}

The probability density of hidden states in operation-dependent CBM policy can be estimated similarly to the way it was done for the integrated decision-making policy and operation-dependent CBM policy using the following Bayes' formula:

$$
\pi_{i v}(t+1)=\frac{\sum_{u \in S_{c i}} q_{i v \gamma_{k}} p_{c_{i}, u, v}^{a_{t}} \pi_{i u}(t)}{\sum_{v \prime \in S_{c i}} \sum_{u \in S_{c i}} q_{i v \prime} \gamma_{k} P_{c_{i}, u, v \prime}^{a_{t}} \pi_{i u}(t)}, \quad v=1, \ldots, M_{c_{i}}
$$

A solution for operation-independent CBM policy can be represented as shown in Figure 36. For a manufacturing system with $m$ stations, where station $c_{i}$ has $M_{c_{i}}$ degradation states, the solution space for optimization of the traditional operationindependent CBM policy consists of up to $\prod_{i=1}^{m}\left(M_{c_{i}}-1\right)$ candidate solutions. Thus, the CBM solution space for a simple 5-station manufacturing system, where each station has

5 degradation states, consists of $4^{5}=1024$ candidate solutions. Since our focus in this study was on a small manufacturing system (see Results section), this problem was solved using complete enumeration. 


\begin{tabular}{|l|l|l|l|}
\hline$S_{c_{1}}$ & $S_{c_{2}}$ & $\cdots$ & $S_{c_{m}}$ \\
\hline
\end{tabular}

Figure 36. Representation of a candidate solution for the operation-independent CBM policy

\section{$5.5 \quad$ Results}

The newly proposed integrated decision-making in the domains of preventive maintenance scheduling and product sequencing with imperfect degradation state observations was tested on an example of a semiconductor manufacturing cluster tool, described in detail in Section 3.4.

In order to assess the performance of the newly proposed optimization methodology, we used the AutoMod software package [140] to simulate a 5-chamber cluster tool, producing 3 types of wafers. Throughout our study, we used 40 simulation replications to evaluate each candidate solution for a maintenance and product sequencing policy. This number was determined by increasing the number of replications until further increase of this number did not result in significant changes in the average profit effects of simulations ran for a wide set of parameters. There was no control over the random number seeds that were used in these replications. It should be noted that, at the end of each simulation, the current system condition is not maintained. 


\subsubsection{Baseline Example}

The parameters used in the baseline example are given in Table 12, Table 13 and Figure 37.

Similar to Chapter 4, let us note that PM times are assumed to be approximately one half of the corresponding RM times. In addition, the night shifts (8pm - 8am) were assumed to be the periods of cheaper maintenance, during which maintenance events were assumed to cost $2 / 5^{\text {th }}$ of a regular maintenance event executed during day shifts (8am-8pm), for both PM and RM interventions. It can also be noted that, based on the corresponding state transition matrices, operations $\mathrm{o}_{2}, \mathrm{o}_{3}, \mathrm{o}_{7}, \mathrm{o}_{9}, \mathrm{o}_{11}$ and $\mathrm{o}_{12}$ degrade the chambers slower than other operations. In addition, analysis of degradation dependent yield shows that operations $\mathrm{o}_{3}, \mathrm{o}_{7}, \mathrm{o}_{9}, \mathrm{o}_{11}$ and $\mathrm{o}_{13}$ are more sensitive to degradation, since their yields decrease more severely as the degradation state of the chamber increases. These points will be important as we analyze the effects of the newly introduced integrated decision-making policy and compare them to the benchmark policies.

As mentioned in the previous section, operation-independent CBM policy was determined through complete enumeration and $S_{\mathrm{CBM}}=\left[\begin{array}{lllll}2 & 3 & 2 & 3 & 3\end{array}\right]$ was found to be the best operation-independent CBM policy for the baseline parameter setting. On the other hand, in order to optimize the integrated decision-making policy, Tabu Search algorithm described in previous section was used. The parameters of the Tabu Search algorithm used in this study were selected in an ad hoc manner and are given in Table 14. One 
should note that general guidelines for a more systematic Tabu Search parameter selection can be found in [141].

$$
\begin{aligned}
& P^{\left(o_{1}\right)}=\left[\begin{array}{ccccc}
0.98 & 0.02 & 0 & 0 & 0 \\
0 & 0.98 & 0.02 & 0 & 0 \\
0 & 0 & 0.98 & 0.02 & 0 \\
0 & 0 & 0 & 0.98 & 0.02 \\
0 & 0 & 0 & 0 & 1
\end{array}\right] P^{\left(o_{2}\right)}=\left[\begin{array}{ccccc}
0.90 & 0.08 & 0.02 & 0 & 0 \\
0 & 0.90 & 0.08 & 0.02 & 0 \\
0 & 0 & 0.90 & 0.08 & 0.02 \\
0 & 0 & 0 & 0.93 & 0.07 \\
0 & 0 & 0 & 0 & 1
\end{array}\right] P^{\left(o_{3}\right)}=\left[\begin{array}{ccccc}
0.93 & 0.05 & 0.02 & 0 & 0 \\
0 & 0.93 & 0.05 & 0.02 & 0 \\
0 & 0 & 0.93 & 0.05 & 0.02 \\
0 & 0 & 0 & 0.97 & 0.03 \\
0 & 0 & 0 & 0 & 1
\end{array}\right] \\
& P^{\left(o_{4}\right)}=\left[\begin{array}{ccccc}
0.94 & 0.04 & 0.015 & 0.005 & 0 \\
0 & 0.94 & 0.04 & 0.015 & 0.005 \\
0 & 0 & 0.94 & 0.04 & 0.02 \\
0 & 0 & 0 & 0.94 & 0.06 \\
0 & 0 & 0 & 0 & 1
\end{array}\right] \quad P^{\left(0_{5}\right)}=\left[\begin{array}{ccccc}
0.98 & 0.015 & 0.005 & 0 & 0 \\
0 & 0.98 & 0.015 & 0.005 & 0 \\
0 & 0 & 0.98 & 0.015 & 0.005 \\
0 & 0 & 0 & 0.98 & 0.02 \\
0 & 0 & 0 & 0 & 1
\end{array}\right] \\
& P^{\left(o_{6}\right)}=\left[\begin{array}{ccccc}
0.95 & 0.04 & 0.01 & 0 & 0 \\
0 & 0.95 & 0.04 & 0.01 & 0 \\
0 & 0 & 0.95 & 0.04 & 0.01 \\
0 & 0 & 0 & 0.95 & 0.05 \\
0 & 0 & 0 & 0 & 1
\end{array}\right] \quad P^{\left(o_{7}\right)}=\left[\begin{array}{ccccc}
0.93 & 0.04 & 0.02 & 0.01 & 0 \\
0 & 0.93 & 0.04 & 0.02 & 0.01 \\
0 & 0 & 0.93 & 0.06 & 0.01 \\
0 & 0 & 0 & 0.97 & 0.03 \\
0 & 0 & 0 & 0 & 1
\end{array}\right] \quad P^{\left(o_{8}\right)}=\left[\begin{array}{ccccc}
0.97 & 0.03 & 0 & 0 & 0 \\
0 & 0.97 & 0.03 & 0 & 0 \\
0 & 0 & 0.97 & 0.03 & 0 \\
0 & 0 & 0 & 0.97 & 0.03 \\
0 & 0 & 0 & 0 & 1
\end{array}\right] \\
& P^{\left(o_{9}\right)}=\left[\begin{array}{ccccc}
0.90 & 0.05 & 0.03 & 0.02 & 0 \\
0 & 0.90 & 0.05 & 0.03 & 0.02 \\
0 & 0 & 0.90 & 0.07 & 0.03 \\
0 & 0 & 0 & 0.90 & 0.10 \\
0 & 0 & 0 & 0 & 1
\end{array}\right] P^{\left(o_{10}\right)}=\left[\begin{array}{ccccc}
0.99 & 0.01 & 0 & 0 & 0 \\
0 & 0.99 & 0.01 & 0 & 0 \\
0 & 0 & 0.99 & 0.01 & 0 \\
0 & 0 & 0 & 0.99 & 0.01 \\
0 & 0 & 0 & 0 & 1
\end{array}\right] P^{\left(o_{11}\right)}=\left[\begin{array}{ccccc}
0.89 & 0.07 & 0.03 & 0.01 & 0 \\
0 & 0.89 & 0.07 & 0.03 & 0.01 \\
0 & 0 & 0.91 & 0.07 & 0.02 \\
0 & 0 & 0 & 0.91 & 0.09 \\
0 & 0 & 0 & 0 & 1
\end{array}\right] \\
& P^{\left(o_{12}\right)}=\left[\begin{array}{ccccc}
0.92 & 0.06 & 0.02 & 0 & 0 \\
0 & 0.92 & 0.06 & 0.02 & 0 \\
0 & 0 & 0.92 & 0.06 & 0.02 \\
0 & 0 & 0 & 0.95 & 0.05 \\
0 & 0 & 0 & 0 & 1
\end{array}\right] \quad P^{\left(o_{13}\right)}=\left[\begin{array}{ccccc}
0.95 & 0.03 & 0.02 & 0 & 0 \\
0 & 0.95 & 0.03 & 0.02 & 0 \\
0 & 0 & 0.95 & 0.03 & 0.02 \\
0 & 0 & 0 & 0.96 & 0.04 \\
0 & 0 & 0 & 0 & 1
\end{array}\right] \quad M P=\left[\begin{array}{ccccc}
0 & 0 & 0 & 0 & 0 \\
1 & 0 & 0 & 0 & 0 \\
0.60 & 0.40 & 0 & 0 & 0 \\
0.60 & 0.30 & 0.10 & 0 & 0 \\
1 & 0 & 0 & 0 & 0
\end{array}\right] \\
& Q=\left[\begin{array}{ccccc}
0.85 & 0.10 & 0.05 & 0 & 0 \\
0.06 & 0.85 & 0.06 & 0.03 & 0 \\
0.03 & 0.06 & 0.85 & 0.06 & 0 \\
0 & 0.05 & 0.10 & 0.85 & 0 \\
1 & 0 & 0 & 0 & 0
\end{array}\right]
\end{aligned}
$$

Figure 37. Operation-specific transition probability matrices, maintenance transition probability matrix and emission probability matrix for integrated decision making with imperfect state observations 
Table 12. Summary of simulation parameters for integrated decision-making with imperfect state observations

\begin{tabular}{|c|c|}
\hline Set of chambers & $C=\left\{c_{1}, c_{2}, \ldots, c_{5}\right\}$ \\
\hline Wafer types & $W=\left\{w_{1}, w_{2}, w_{3}\right\}$ \\
\hline $\begin{array}{l}\text { Number of wafers for each } \\
\text { wafer type }\end{array}$ & $\begin{array}{l}F=12, F_{w_{1}}=4, F_{w_{2}}=4, F_{w_{3}}=4 \text { (These are the number of lots and } \\
\text { each lot consists of } 25 \text { wafers, hence } 100 \text { wafers of each wafer type are fed } \\
\text { into the system) }\end{array}$ \\
\hline Wafer type sequence & {$\left[d_{1}, d_{2}, \ldots, d_{12}\right]=\left[w_{1}, w_{2}, w_{3}, w_{1}, w_{2}, w_{3}, w_{1}, w_{2}, w_{3}, w_{1}, w_{2}, w_{3}\right]$} \\
\hline Production goal (wafers) & $N_{w_{1}}=N_{w_{2}}=N_{w_{3}}=85$ \\
\hline Set of operations & $O=\left\{o_{1}, o_{2}, \ldots, o_{13}\right\}$ \\
\hline Operation cycle times (min) & $\begin{array}{l}{[10,20,15,15,20,20,10,25,10,15,10,10]} \\
o_{w_{1}}=\left[o_{1}, o_{2}, o_{3}, o_{4}, o_{13}, o_{5}\right]\end{array}$ \\
\hline $\begin{array}{l}\text { Operation sequences for each } \\
\text { wafer type }\end{array}$ & $\begin{aligned} O_{w_{2}} & =\left[o_{6}, o_{3}, o_{7}, o_{11}, o_{12}\right] \\
o_{w_{3}} & =\left[o_{8}, o_{9}, o_{10}, o_{11}, o_{6}\right] \\
O_{c_{1}} & =\left\{o_{1}, o_{2}, o_{4}, o_{5}, o_{6}, o_{7}, o_{8}, o_{9}\right\} \\
o_{c_{2}} & =\left\{o_{1}, o_{2}, o_{3}, o_{4}, o_{6}, o_{8}, o_{9}, o_{10}\right\}\end{aligned}$ \\
\hline $\begin{array}{l}\text { Operations executable in each } \\
\text { chamber }\end{array}$ & $\begin{array}{l}O_{c_{3}}=\left\{o_{3}, o_{4}, o_{5}, o_{7}, o_{9}, o_{10}, o_{11}\right\} \\
o_{c_{4}}=\left\{o_{2}, o_{8}, o_{12}, o_{13}\right\} \\
O_{c_{5}}=\left\{o_{1}, o_{5}, o_{6}, o_{7}, o_{11}, o_{12}\right\}\end{array}$ \\
\hline Degradation state space & $S_{c_{1}}=S_{c_{2}}=\cdots=S_{c_{5}}=\{1,2, \ldots, 5\}$ \\
\hline $\begin{array}{l}\text { Distributions of preventive } \\
\text { maintenance times for each } \\
\text { chamber ( } \mathrm{min} \text { ) }\end{array}$ & {$[U(31,49), U(51,69), U(41,59), U(21,39), U(21,39)]$} \\
\hline Periods of cheaper maintenance & 8:00pm - 8:00am each day \\
\hline $\begin{array}{l}\text { Reactive maintenance times for } \\
\text { each chamber ( } \mathrm{min} \text { ) }\end{array}$ & {$[U(62,98), U(102,138), U(82,118), U(42,78), U(42,78)]$} \\
\hline Reward parameters (\$) & $R_{w_{1}}=50, \quad R_{w_{2}}=25, \quad R_{w_{3}}=10$ \\
\hline Costs (\$) & $c_{p}=50, \quad c_{r}=250, \quad c_{c p}=20, \quad c_{c r}=100, p_{w_{1}}=20, p_{w_{2}}=10, \quad p_{w_{3}}=4$ \\
\hline Alpha $(\alpha)$ & 0.70 \\
\hline
\end{tabular}


Table 13. Probability of success ("yield") for each operation for integrated decisionmaking with imperfect state observations

\begin{tabular}{|c|ccccc|}
\cline { 2 - 6 } \multicolumn{1}{c|}{} & State 1 & State 2 & State 3 & State 4 & State 5 \\
\hline $\boldsymbol{o}_{\mathbf{1}}$ & 1 & 0.9606 & 0.8295 & 0.8122 & 0.7938 \\
\hline $\boldsymbol{o}_{\mathbf{2}}$ & 1 & 0.9900 & 0.9327 & 0.9132 & 0.8896 \\
\hline $\boldsymbol{o}_{\mathbf{3}}$ & 1 & 0.9224 & 0.6022 & 0.5677 & 0.4935 \\
\hline $\boldsymbol{o}_{\mathbf{4}}$ & 1 & 0.9801 & 0.8877 & 0.8619 & 0.8284 \\
\hline $\boldsymbol{o}_{\mathbf{5}}$ & 1 & 0.9512 & 0.8650 & 0.7643 & 0.7438 \\
\hline $\boldsymbol{o}_{\mathbf{6}}$ & 1 & 0.9276 & 0.9110 & 0.8946 & 0.8525 \\
\hline $\boldsymbol{o}_{\mathbf{7}}$ & 1 & 0.9317 & 0.8386 & 0.5721 & 0.5062 \\
\hline $\boldsymbol{o}_{\mathbf{8}}$ & 1 & 0.9631 & 0.8301 & 0.7737 & 0.7316 \\
\hline $\boldsymbol{o}_{\mathbf{9}}$ & 1 & 0.9360 & 0.9042 & 0.7430 & 0.6866 \\
\hline $\boldsymbol{o}_{\mathbf{1 0}}$ & 1 & 0.9511 & 0.9397 & 0.9284 & 0.8991 \\
\hline $\boldsymbol{o}_{\mathbf{1 1}}$ & 1 & 0.9500 & 0.8758 & 0.8418 & 0.7084 \\
\hline $\boldsymbol{o}_{\mathbf{1 2}}$ & 1 & 0.9739 & 0.9605 & 0.8880 & 0.8603 \\
\hline $\boldsymbol{o}_{\mathbf{1 3}}$ & 1 & 0.9276 & 0.7848 & 0.7169 & 0.6354 \\
\hline
\end{tabular}

Table 14. Parameters of Tabu Search for integrated decision-making with imperfect state observations

\begin{tabular}{|l|l|}
\hline Parameters of Tabu Search & Value \\
\hline Maximum iterations & 20 \\
\hline Tabu list length & 5 \\
\hline Neighbors for product type sequence & 10 \\
\hline Neighbors for maintenance triggering states & 15 \\
\hline Replication number to find expected profit & 40 \\
\hline
\end{tabular}

From Figure 38, it is immediately visible that the integrated decision-making policy results in a higher expected profit, when compared to the traditional operationindependent $\mathrm{CBM}$ policy and the operation-dependent CBM policy. There are several reasons for this performance improvement brought by the proposed integrated decisionmaking method. Firstly, the benefits of considering operation-dependent degradation 
dynamics and postulating an operation-dependent maintenance policy were apparent, similarly to what was found in [7] and [8]. Namely, for the best integrated decisionmaking policy, it was observed that the maintenance triggering states $S_{\mathrm{C}_{1}}^{\mathrm{o}_{2}}, \mathrm{~S}_{\mathrm{c}_{1}}^{\mathrm{o}_{7}}, \mathrm{~S}_{\mathrm{C}_{1}}^{\mathrm{o}_{9}}, \mathrm{~S}_{\mathrm{C}_{3}}^{\mathrm{o}_{11}}$ and $\mathrm{S}_{\mathrm{C}_{5}}^{\mathrm{o}_{11}}$ are higher (allowing more degradation) compared to the traditional, operationindependent $\mathrm{CBM}$ policy. As mentioned before, operations $\mathrm{o}_{2}, \mathrm{o}_{7}, \mathrm{o}_{9}$ and $\mathrm{o}_{11}$ degrade the chambers slower than others and the optimized operation-mode dependent maintenance triggering states yielded by the procedure proposed in this chapter support the intuition that less frequent maintenance should be allowed for slower degrading operations. In addition, since operations $\mathrm{o}_{7}, \mathrm{o}_{9}$ and $\mathrm{o}_{11}$ are involved in the manufacturing of cheaper (less rewarding) wafers, the corresponding optimized maintenance triggering states also conform with the intuition that as the wafer reward decreases, the effect of completing that wafer successfully on the profit decreases, thus favoring later triggering of maintenance.

In this study, as mentioned in Section 5.4.4, for the benchmark policies, the action $a_{t+1}$ to be taken at time $t+1$, is determined based on the information obtained from the distribution $\pi_{i}(t+1)$ of hidden states of station $c_{i}$ at time $t+1$. A much simpler practice, that is commonly implemented in manufacturing fabs, is determining the action at time $t+1$ according to the highest likelihood state based on the observed symbol. However, as can be seen from the leftmost bar in Figure 38, this approach yields much lower expected profits when compared to the proposed integrated decision-making policy. 
Clear benefits of the ability to adjust product sequencing within the newly proposed decision-making scheme can be seen in Figure 39. One can observe in Figure 39 (a) that the integrated decision-making policy not only decreases the total number of PM events, but also increases the number of the PM events occurring in the cheaper maintenance periods, when compared to the benchmark policies. The reason for this is that, by allowing changes in the product sequence, the integrated decision-making policy takes advantage of the cheaper maintenance periods and rearranges the product sequencing in a way that more PM events are pushed to take place during those periods. This is a significant feature which contributed to the beneficial cost effects of the decision-making procedure proposed in this chapter, as it did in Chapter 4, which is not available in the fragmented maintenance and product sequencing decision-making schemes, traditionally pursued in manufacturing today. 


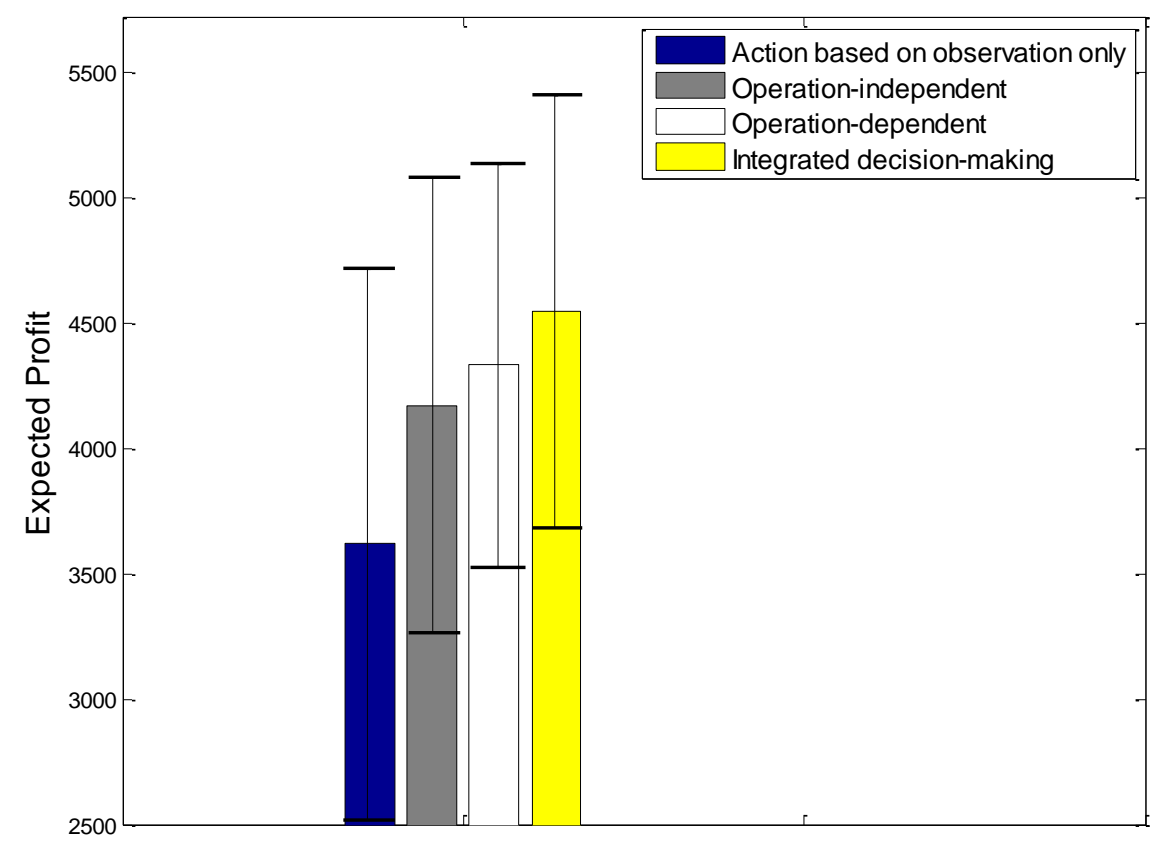

Figure 38. Comparison of expected profits for the integrated decision-making policy, operation-dependent CBM policy and the traditional operation-independent CBM policy with imperfect state observations

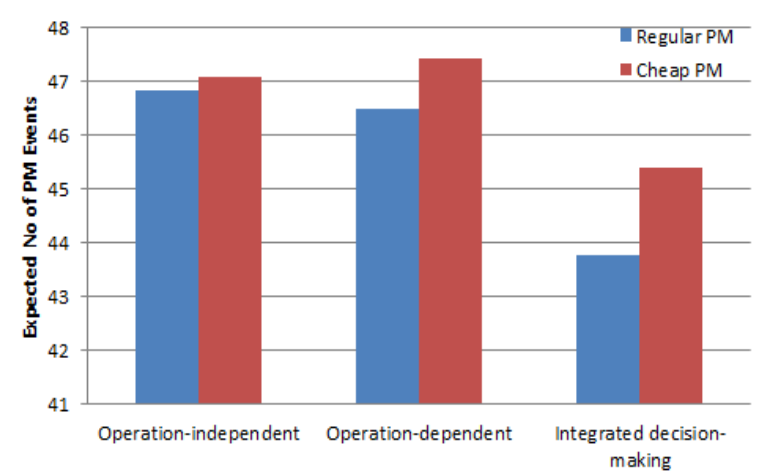

(a) Expected number of PM events

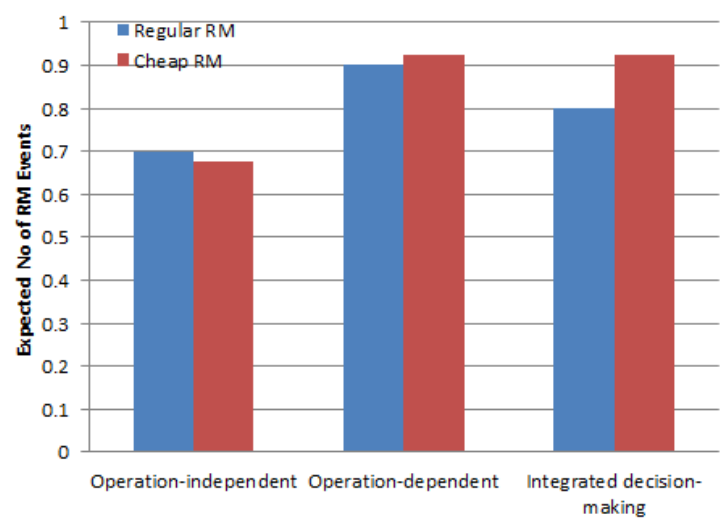

(b) Expected number of RM events

Figure 39. Comparison between the expected numbers of maintenance events conducted during the expensive and cheap maintenance periods for a system with imperfect state observations 
To gain better understanding of the newly proposed integrated decision-making policy, we studied the influences of maintenance threshold $(\alpha)$, production goal $\left(\mathrm{N}_{\mathrm{w}_{\mathrm{j}}}\right)$ parameters, efficacy of maintenance execution and uncertainty in emission matrix on the resulting decisions and their effects. Results of these studies are enclosed below.

\subsubsection{Influence of maintenance probability threshold ( $\alpha)$}

As explained in Section 5.3, the execution of a preventive maintenance event is dependent on whether the probability that degradation progressed beyond $S_{c_{i}}^{o_{k}}$ is greater than some threshold $\alpha$ or not.

In this section, we evaluate the performance of the integrated decision-making policy under varying values of $\alpha$. It is visible from Figure 40 that the expected profits obtained using the integrated decision-making policy are consistently higher than the profits obtained by the benchmark policies. In addition, it can be observed in Figure 41 that the percent improvement of the integrated decision-making policy over benchmark policies is higher for the smallest $\alpha$ value and lower and similar for higher $\alpha$ values. The reason for this is that when $\alpha$ is small, the system is prone to having more PM events and hence the capabilities of integrated decision-making becomes crucial in keeping both the maintenance and scrap cost low. The benchmark policies also aim to keep the maintenance costs low but are not able to reduce the scrap at the same time. As $\alpha$ increases, the probability of triggering a maintenance event becomes lower and hence the impact of the capability of integrated decision-making policy in keeping the scrap cost low reduces. 
The p-values calculated through a paired t-test to evaluate the statistical significance of the outperformance of the proposed method over the benchmark policies are given in Table 15. The proposed integrated decision-making policy outperforms both the operation-independent CBM policy and operation-dependent CBM policy with statistical significance in all instances.

The p-values for the same decision-making policy but for varying $\alpha$ values are given in Table 16.

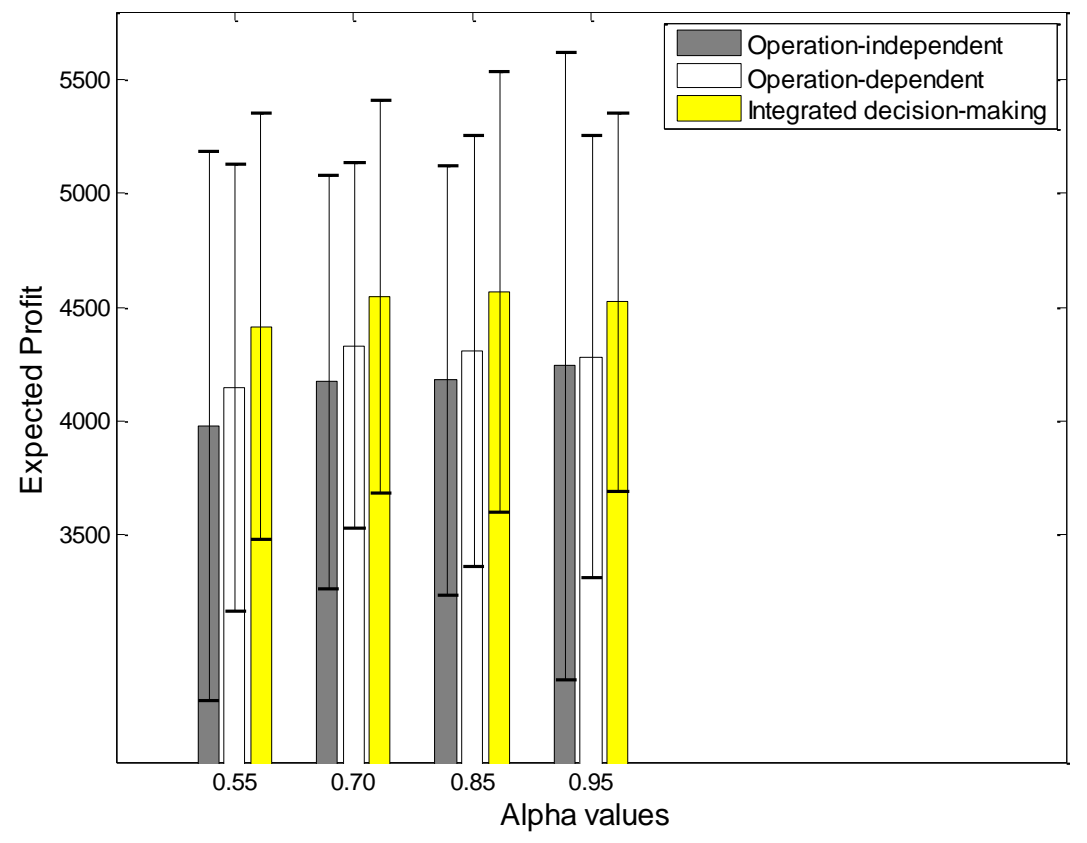

Figure 40. Comparison of expected profits for different alpha $(\alpha)$ values (black lines denote the $-/+2 \sigma$ limits of the simulation outcomes) for the integrated decision-making policy, operation-dependent CBM policy and the traditional operation-independent CBM policy 


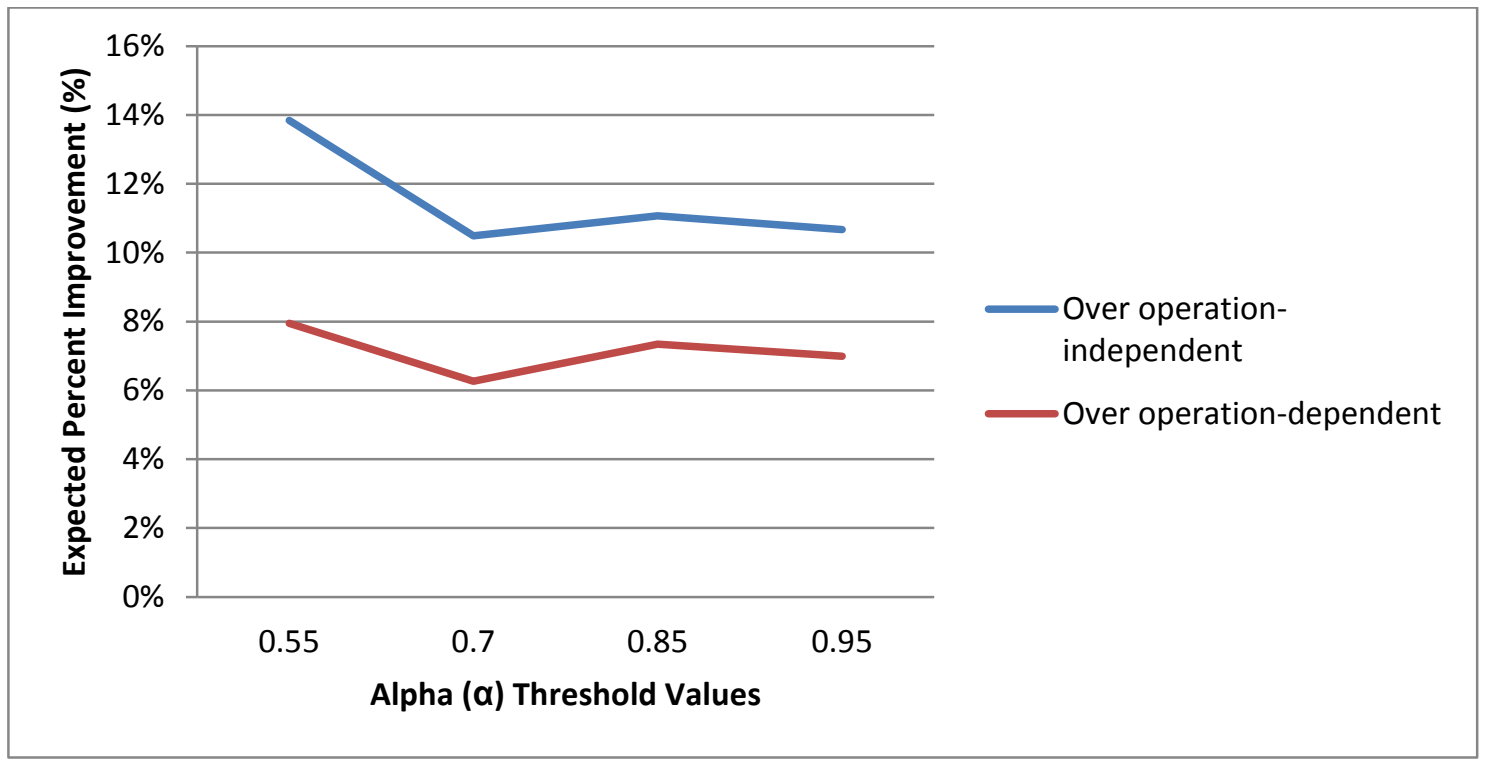

Figure 41. Percent improvement obtained by the integrated decision-making policy over the benchmark policies for different alpha $(\alpha)$ values

Table 15. p-values for different alpha $(\alpha)$ threshold values

\begin{tabular}{|c|c|c|c|c|}
\hline & $\alpha=0.55$ & $\alpha=0.70$ & $\alpha=0.85$ & $\alpha=0.95$ \\
\hline $\begin{array}{c}\text { Integrated decision-making } \\
\text { over } \\
\text { Operation-independent }\end{array}$ & 0.0006 & 0.0013 & 0.0022 & 0.0440 \\
\hline $\begin{array}{c}\text { Integrated decision-making } \\
\text { over } \\
\text { Operation-dependent }\end{array}$ & 0.0189 & 0.0595 & 0.0177 & 0.0261 \\
\hline
\end{tabular}

Table 16. p-values for the same decision-making policy over varying alpha $(\alpha)$ threshold values

\begin{tabular}{|c|c|c|c|}
\cline { 2 - 4 } \multicolumn{1}{c|}{} & $\begin{array}{c}\alpha=0.55 \\
\text { vs } \\
\alpha=0.70\end{array}$ & $\begin{array}{c}\alpha=0.70 \\
\text { vs } \\
\alpha=0.85\end{array}$ & $\begin{array}{c}\alpha=0.85 \\
\text { vs } \\
\alpha=0.95\end{array}$ \\
\hline Operation-independent & 0.0836 & 0.9364 & 0.6362 \\
\hline Operation-dependent & 0.0805 & 0.8134 & 0.8159 \\
\hline Integrated decision-making & 0.2299 & 0.8090 & 0.5975 \\
\hline
\end{tabular}




\subsubsection{Influence of emission probability matrices $(Q)$}

As explained in detail in Section 5.3, the major improvement compared to the research presented in Chapter 4 is that the degradation state of the system is not perfectly observable anymore and must be inferred from the available observations. The emission probability matrix $(Q)$ provides the probabilistic relations between the hidden degradation states and the available observations.

In this section, we will investigate the performance of the integrated decisionmaking policy under various emission probability matrices. It is assumed that there are five observation symbols that can be used to infer the actual degradation states which

makes these matrices 5 by 5 , with $q_{i j}$ denoting the probability of observing symbol $j$ if actual state is $i$. Matrices $Q 1-Q 5$, shown in Figure 42, are selected in a way that as we go from $Q 1$ to $Q 5$, the relation between the observed symbol and the actual degradation state becomes more and more deterministic (entropies of the matrices become smaller as we go from $Q 1$ to $Q 5)$. In $Q 5$ case, the relation is completely deterministic, i.e. given an observation symbol, one can identify the actual degradation state with probability 1 .

$$
\begin{gathered}
Q 1=\left[\begin{array}{ccccc}
0.55 & 0.3 & 0.15 & 0 & 0 \\
0.18 & 0.55 & 0.18 & 0.09 & 0 \\
0.09 & 0.18 & 0.55 & 0.18 & 0 \\
0 & 0.15 & 0.3 & 0.55 & 0 \\
0 & 0 & 0 & 0 & 1
\end{array}\right] \quad Q 2=\left[\begin{array}{ccccc}
0.7 & 0.2 & 0.1 & 0 & 0 \\
0.13 & 0.7 & 0.13 & 0.04 & 0 \\
0.04 & 0.13 & 0.7 & 0.13 & 0 \\
0 & 0.1 & 0.2 & 0.7 & 0 \\
0 & 0 & 0 & 0 & 1
\end{array}\right] \quad Q 3= \\
{\left[\begin{array}{cccccc}
0.85 & 0.10 & 0.05 & 0 & 0 \\
0.06 & 0.85 & 0.06 & 0.03 & 0 \\
0.0 .3 & 0.06 & 0.85 & 0.06 & 0 \\
0 & 0.05 & 0.10 & 0.85 & 0 \\
0 & 0 & 0 & 0 & 1
\end{array}\right] \quad Q 4=\left[\begin{array}{ccccccc}
0.95 & 0.04 & 0.01 & 0 & 0 \\
0.02 & 0.95 & 0.02 & 0.01 & 0 \\
0.01 & 0.02 & 0.95 & 0.02 & 0 \\
0 & 0.01 & 0.04 & 0.95 & 0 \\
0 & 0 & 0 & 0 & 1
\end{array}\right] \quad Q 5=\left[\begin{array}{llllll}
1 & 0 & 0 & 0 & 0 \\
0 & 1 & 0 & 0 & 0 \\
0 & 0 & 1 & 0 & 0 \\
0 & 0 & 0 & 1 & 0 \\
0 & 0 & 0 & 0 & 1
\end{array}\right]}
\end{gathered}
$$

Figure 42. Emission probability matrices used for sensitivity analysis 
As observed in Figure 43, the integrated decision-making policy outperforms the benchmark CBM policies for various emission probability matrices. The p-values presented in Table 17 show that the outperformance of the integrated decision-making policy over traditional operation-independent CBM policy is statistically significant for all cases but $Q 1$. For the operation-dependent CBM policy, it is statistically significant for all cases but $Q 1$ and $Q 2$. These findings are consistent with the trend observed in Figure 44. As mentioned above, when we go from $Q 5$ to $Q 1$, estimation of the actual degradation states from the observed symbols become more and more stochastic, making the maintenance decisions more unreliable. Therefore, the benefits of the integrated decision-making over the benchmark policies would be low for these more stochastic cases.

As mentioned above, emission probability matrix $Q 5$ represents the case where we can determine the actual degradation state with certainty for a given observation symbol. This case is identical to the problem addressed in Chapter 4 where the degradation states were perfectly observable. It can be observed in Figure 45 that the expected profits obtained by the integrated decision-making policy with imperfect degradation states using emission probability matrix $Q 5$ are almost the same as the expected profits obtained by the integrated decision-making policy with perfect degradation-state observations (see Figure 19), which is confirmed by a p-value of 0.756 . 


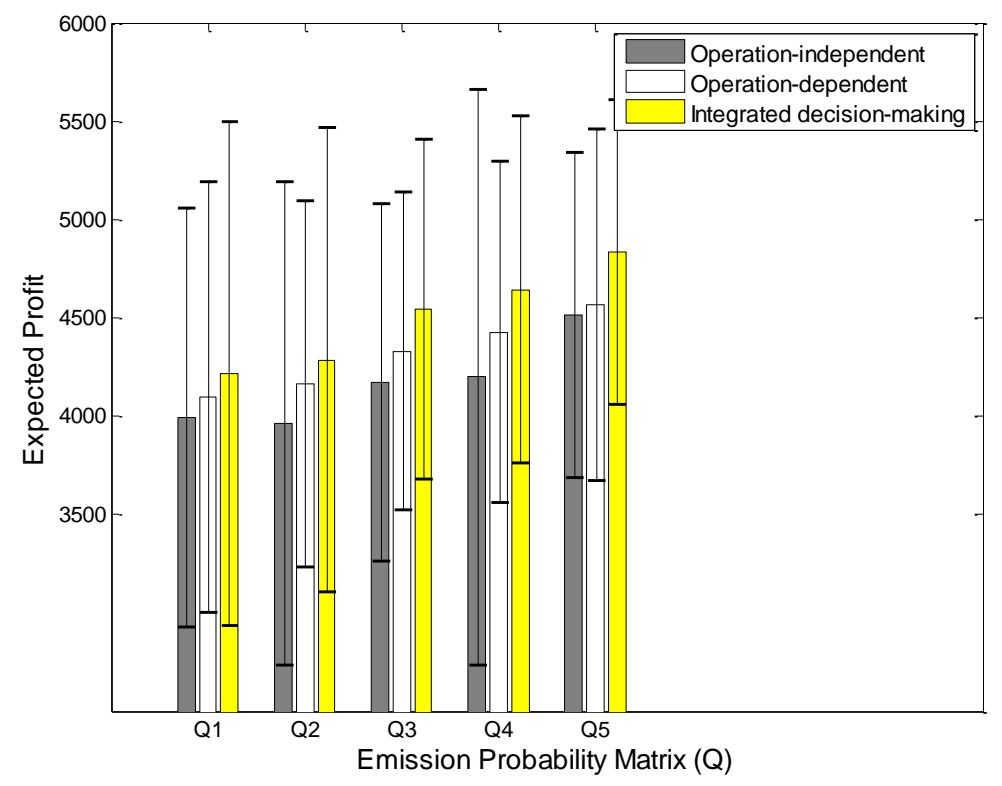

Figure 43. Comparison of expected profits for different emission probability matrices (black lines denote the $-/+2 \sigma$ limits of the simulation outcomes) for the integrated decision-making policy, operation-dependent $\mathrm{CBM}$ policy and the traditional operationindependent CBM policy

Table 17. p-values for different emission probability matrices

\begin{tabular}{|c|c|c|c|c|c|}
\cline { 2 - 5 } \multicolumn{1}{c|}{} & Q1 & Q2 & Q3 & Q4 & Q5 \\
\hline $\begin{array}{c}\text { Integrated decision-making } \\
\text { over } \\
\text { Operation-independent }\end{array}$ & 0.1138 & 0.0167 & 0.0013 & 0.0011 & 0.0021 \\
\hline $\begin{array}{c}\text { Integrated decision-making } \\
\text { over }\end{array}$ & 0.4132 & 0.3515 & 0.0595 & 0.0159 & 0.0066 \\
Operation-dependent & & & & \\
\hline
\end{tabular}




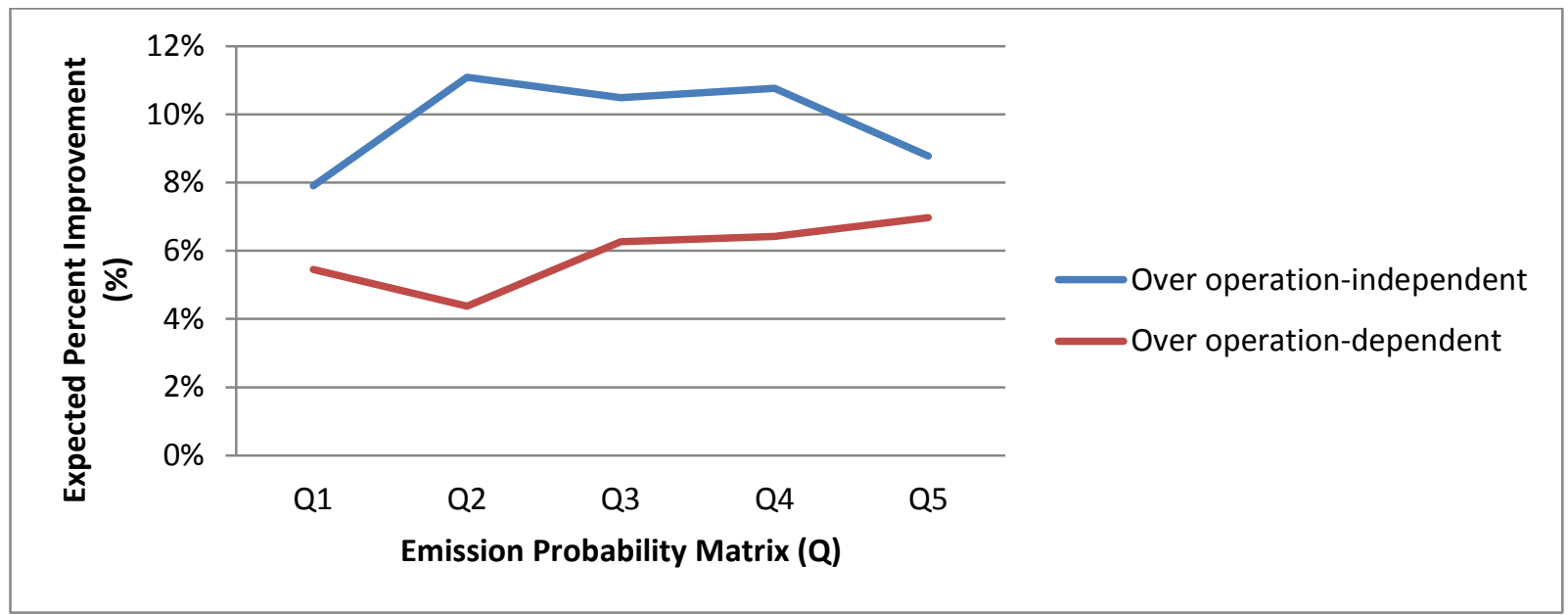

Figure 44. Percent improvement obtained by the integrated decision-making policy over the benchmark policies for different emission probability matrices

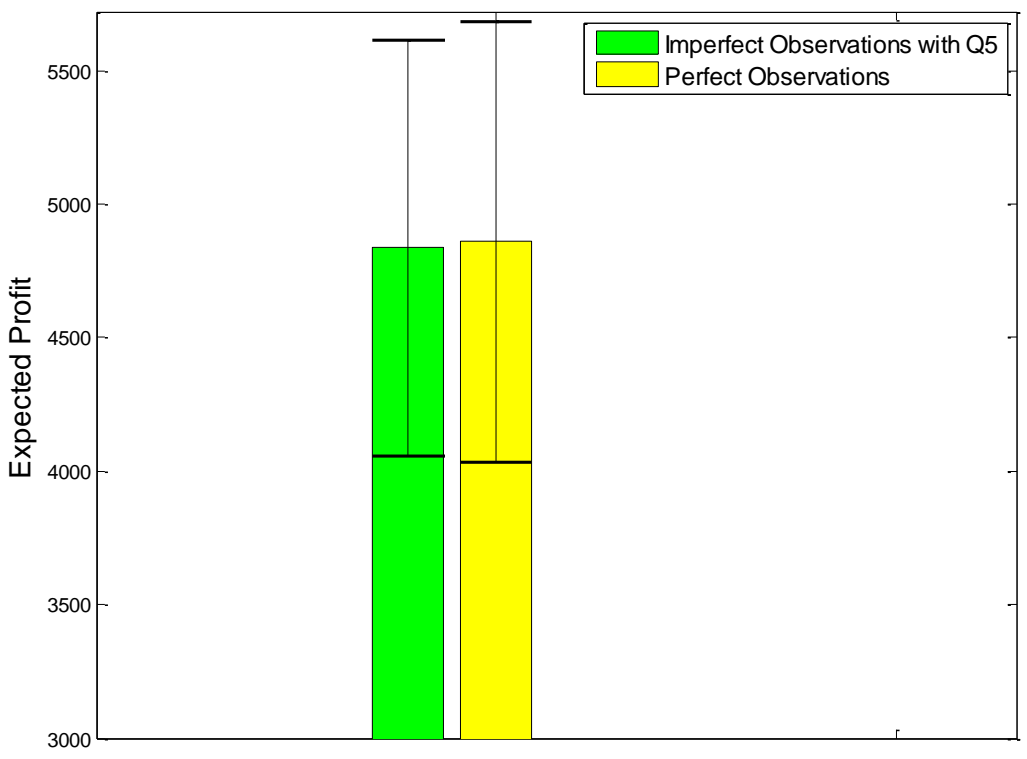

Figure 45. Comparison of expected profits obtained by the integrated decision-making policy with imperfect observations using emission probability matrix $Q 5$ and with perfect observations (black lines denote the $-/+2 \sigma$ limits of the simulation outcomes) 


\subsubsection{Influence of production goals $\left(N_{w_{j}}\right)$}

Let us now assess the performance of the newly introduced integrated decisionmaking methodology under increasingly demanding production goals ${ }^{18}$. The expected profits for the newly proposed and benchmark decision-making schemes are shown in Figure 46 and, once again, the integrated decision-making policy consistently yields higher expected profits, when compared to the benchmark policies. The p-values given in Table 18 show that integrated decision-making policy outperforms the benchmark policies significantly.

This can be explained by the fact that as the production goals increase, avoiding unscheduled downtime and production penalty costs by preventing the production of scrap wafers becomes increasingly influential on the effects of any decision-making policy. The integrated decision-making policy jointly optimizes product sequencing and operation-specific maintenance triggering states and thus offers more freedom in the operational decision-making. For example, for the operations that are executed in the completion of the wafers for which we have a higher production goal, the integrated decision-making policy can suggest earlier triggering of maintenance, thus reducing the number of scrap wafers and increasing the likelihood of reaching that production goal. It can also compensate for the consequent increase in the number of preventive maintenance events by suggesting later triggering of maintenance events for other

\footnotetext{
${ }^{18}$ I.e. as one increases the production goals $N_{w_{j}}$ for each product type in the cost functions (1), (2) and (3).
} 
operations. In addition, by changing the product sequence, the integrated decisionmaking has the flexibility to assign less sensitive operations to more degraded chambers, thus reducing the number of scrap wafers, and potentially postponing the maintenance of those machines towards periods of cheaper maintenance. The traditional operationindependent CBM policy does not have any of these flexibilities and the operationdependent CBM policy does not have the flexibility of product sequencing and hence as the production goals increase, in order to reduce the number of scrap wafers, the number of maintenance events, and hence maintenance costs, increase more dramatically than in the case of the integrated decision-making policy.

It is therefore logical that as the production goals increase, the relative benefits of the integrated decision-making policy over the benchmark CBM policies consistently increase, as can be seen in Figure 47. 


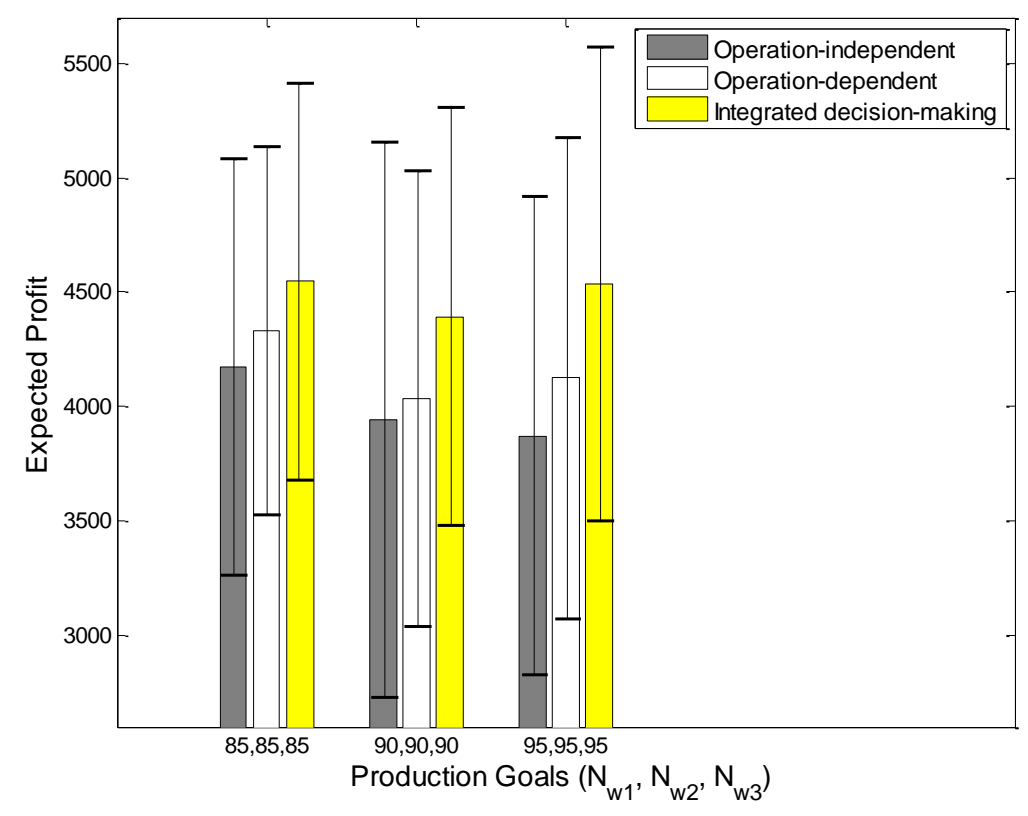

Figure 46. Comparison of expected profits for different production goals (black lines denote the $-/+2 \sigma$ limits of the simulation outcomes) for the integrated decision-making policy, operation-dependent CBM policy and the traditional operation-independent CBM policy with imperfect state observations

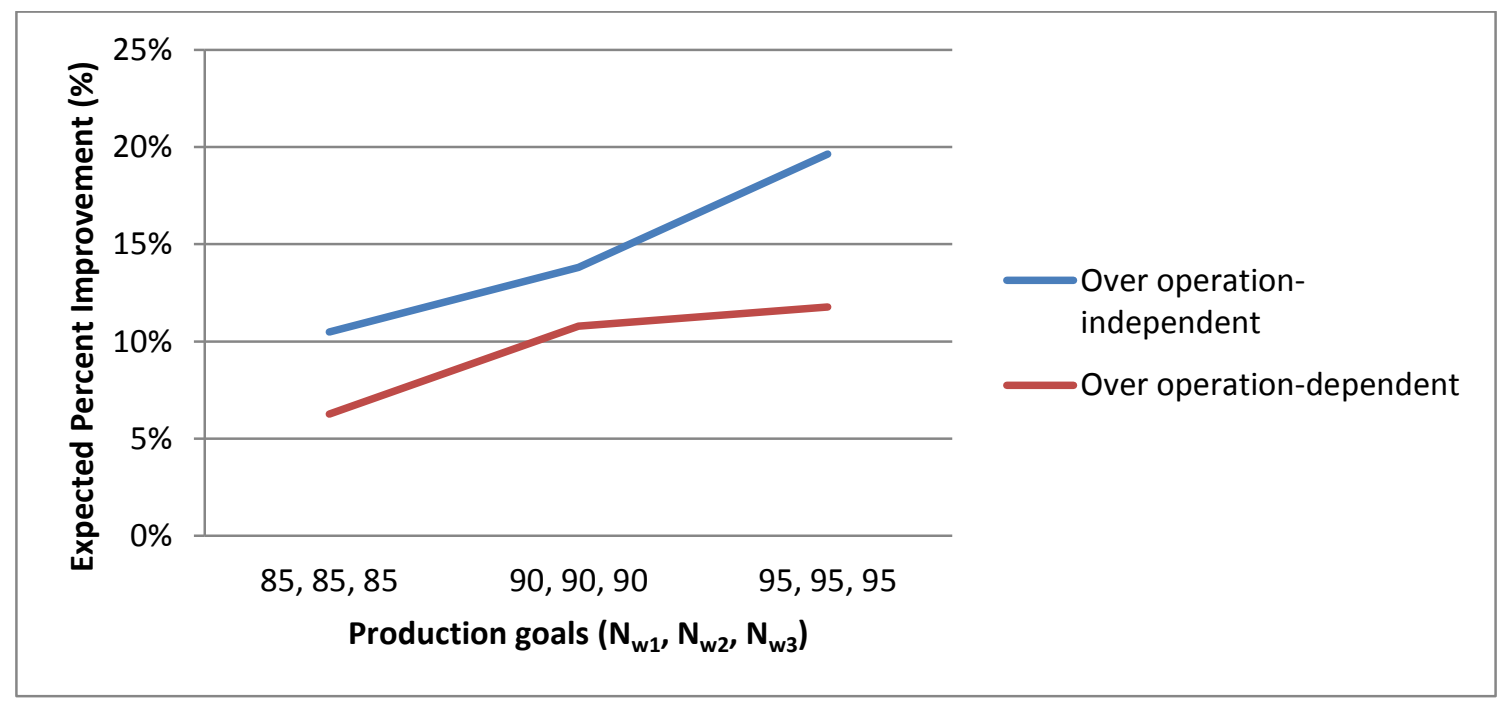

Figure 47. Percent improvement over benchmark policies obtained by the integrated decision-making policy with imperfect state observations for different production goals 
Table 18. p-values for different production goals $\left(N_{w_{1}}, N_{w_{2}}, N_{w_{3}}\right)$ for a system with imperfect state observations

\begin{tabular}{|c|c|c|c|}
\cline { 2 - 4 } \multicolumn{1}{c|}{} & $85,85,85$ & $90,90,90$ & $95,95,95$ \\
\hline $\begin{array}{c}\text { Integrated decision-making } \\
\text { over }\end{array}$ & 0.0013 & 0.0001 & 0.0001 \\
\hline $\begin{array}{c}\text { Operation-independent } \\
\text { Integrated decision-making } \\
\text { over }\end{array}$ & 0.0595 & 0.0029 & 0.0011 \\
\hline Operation-dependent & &
\end{tabular}

\subsubsection{Influence of probabilities that preventive maintenance actions restore the perfect condition of the maintained system (MP)}

As mentioned in the problem description in Section 5.3, we acknowledge in this study that imperfect maintenance may happen. In this section, we will evaluate the impacts of the effectiveness of maintenance operations on the expected profits yielded by different decision-making policies.

Various effectiveness levels of PM interventions are tested using different Markov transition matrices corresponding to the condition-state recoveries induced by PM events. Figure 48 lists the PM-related Markov transition matrices tested in this study. Generally speaking, these matrices express the effectiveness of maintenance operations via the expected equipment condition after a maintenance event is executed. Matrices MP1 - MP5 are selected in such a way that, regardless of the state at which maintenance is invoked, as we go from MP1 to MP5, we see increasingly efficient PM interventions, with increasing probabilities that PM returns the maintained system to better states 
(closer to the "as good as new" state). Note that MP5 corresponds to perfect maintenance operations that always return the maintained system to the "as good as new" state.

$$
\begin{gathered}
M P_{1}=\left[\begin{array}{ccccc}
1 & 0 & 0 & 0 & 0 \\
1 & 0 & 0 & 0 & 0 \\
0.20 & 0.80 & 0 & 0 & 0 \\
0.20 & 0.60 & 0.20 & 0 & 0 \\
1 & 0 & 0 & 0 & 0
\end{array}\right] M P_{2}=\left[\begin{array}{ccccc}
1 & 0 & 0 & 0 & 0 \\
1 & 0 & 0 & 0 & 0 \\
0.40 & 0.60 & 0 & 0 & 0 \\
0.40 & 0.45 & 0.15 & 0 & 0 \\
1 & 0 & 0 & 0 & 0
\end{array}\right] M P_{3}=\left[\begin{array}{ccccc}
1 & 0 & 0 & 0 & 0 \\
1 & 0 & 0 & 0 & 0 \\
0.60 & 0.40 & 0 & 0 & 0 \\
0.60 & 0.30 & 0.10 & 0 & 0 \\
1 & 0 & 0 & 0 & 0
\end{array}\right] \\
M P_{4}=\left[\begin{array}{cccccc}
1 & 0 & 0 & 0 & 0 \\
1 & 0 & 0 & 0 & 0 \\
0.80 & 0.20 & 0 & 0 & 0 \\
0.80 & 0.15 & 0.05 & 0 & 0 \\
1 & 0 & 0 & 0 & 0
\end{array}\right] M P_{5}=\left[\begin{array}{lllll}
1 & 0 & 0 & 0 & 0 \\
1 & 0 & 0 & 0 & 0 \\
1 & 0 & 0 & 0 & 0 \\
1 & 0 & 0 & 0 & 0 \\
1 & 0 & 0 & 0 & 0
\end{array}\right]
\end{gathered}
$$

Figure 48. Perfect maintenance probabilities used for sensitivity analysis of the integrated decision-making policy with imperfect state observations

The expected profits obtained for various levels of maintenance efficacies are shown in Figure 49, while the percent improvements obtained with the integrated decision-making policy over the benchmark policies are given in Figure 50.

From Figure 49, we can once again observe that for all levels of PM efficacy, the integrated decision-making policy results in a higher expected profit, when compared to the benchmark policies. In addition, from Figure 50 one can see that the percentual improvements obtained by the integrated decision-making policy over the benchmark policies first rise and then decrease. This phenomenon can be explained through the following reasoning. When the maintenance efficacy is lower, the maintenance triggering thresholds are not reliable in either of the benchmark policies or the integrated decisionmaking policy and hence the benefits of product sequencing become more pronounced. 
However, as the maintenance efficacy keeps increasing, the maintenance decisions are applied more and more effectively and hence the relative advantage of the integrated decision-making policy due to the ability to rearrange the product sequence decreases, resulting in the trend we see in Figure 50. It can also be observed from the same figure that the percentual improvements of the integrated decision-making policy over the operation-independent CBM policy are always higher than the percentual improvements over the operation-dependent CBM policy. This is due to the fact that even though the integrated decision-making policy's advantage via the capability of product sequencing reduces, it still has an added advantage over the traditional operation-independent CBM policy by using operation-dependent maintenance triggering states.

The p-values calculated through a paired t-test are presented in Table 19. Integrated decision-making policy yields significantly better results over operationindependent CBM policy for all but the lowest maintenance efficacy values. Following the trend given in Figure 50, statistical significance of integrated decision-making policy over operation-dependent CBM policy decreases as the maintenance efficacy increases.

Table 19. p-values for different probabilities of perfect maintenance for a system with imperfect state observations

\begin{tabular}{|c|c|c|c|c|c|}
\cline { 2 - 5 } \multicolumn{1}{c|}{} & MP1 & MP2 & MP3 & MP4 & MP5 \\
\hline $\begin{array}{c}\text { Integrated decision-making } \\
\text { over }\end{array}$ & 0.064 & 0.0019 & 0.0013 & 0.0007 & 0.0461 \\
\hline $\begin{array}{c}\text { Operation-independent } \\
\text { Integrated decision-making } \\
\text { over }\end{array}$ & 0.1446 & 0.0368 & 0.0595 & 0.1134 & 0.1706 \\
\hline \begin{tabular}{c} 
Operation-dependent \\
\hline
\end{tabular} & & & & & \\
\hline
\end{tabular}




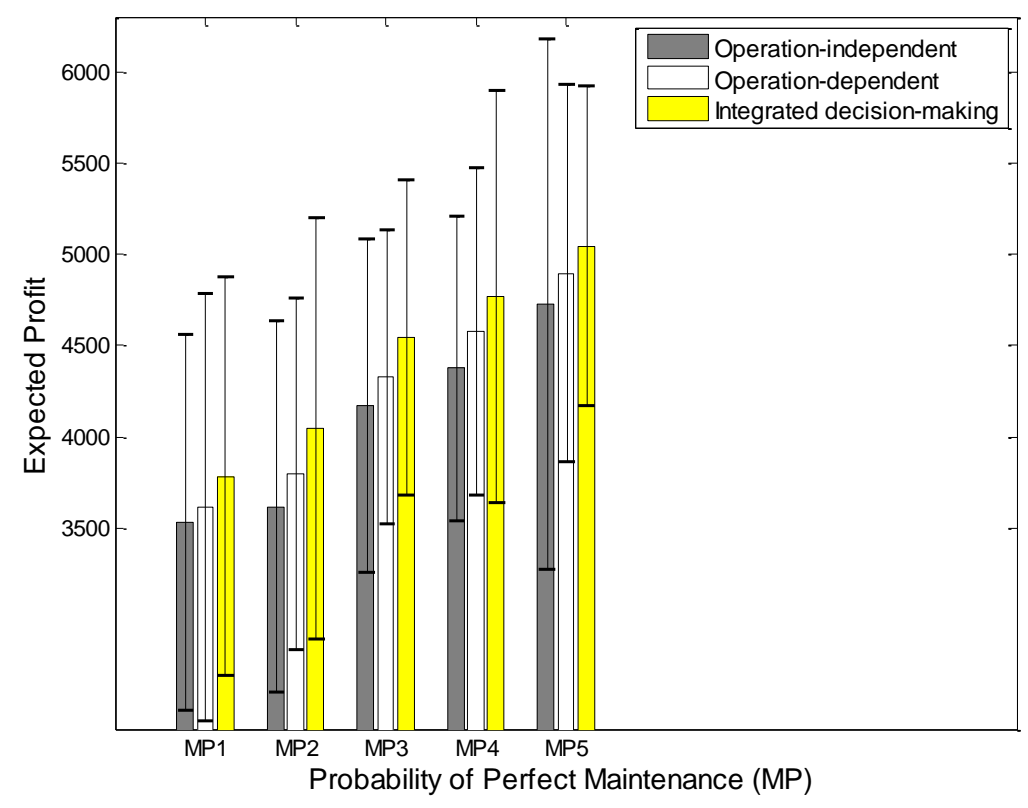

Figure 49. Comparison of expected profits for different probabilities of perfect maintenance (black lines denote the $-/+2 \sigma$ limits of the simulation outcomes) for the integrated decision-making policy, operation-dependent CBM policy and the traditional operation-independent CBM policy with imperfect state observations

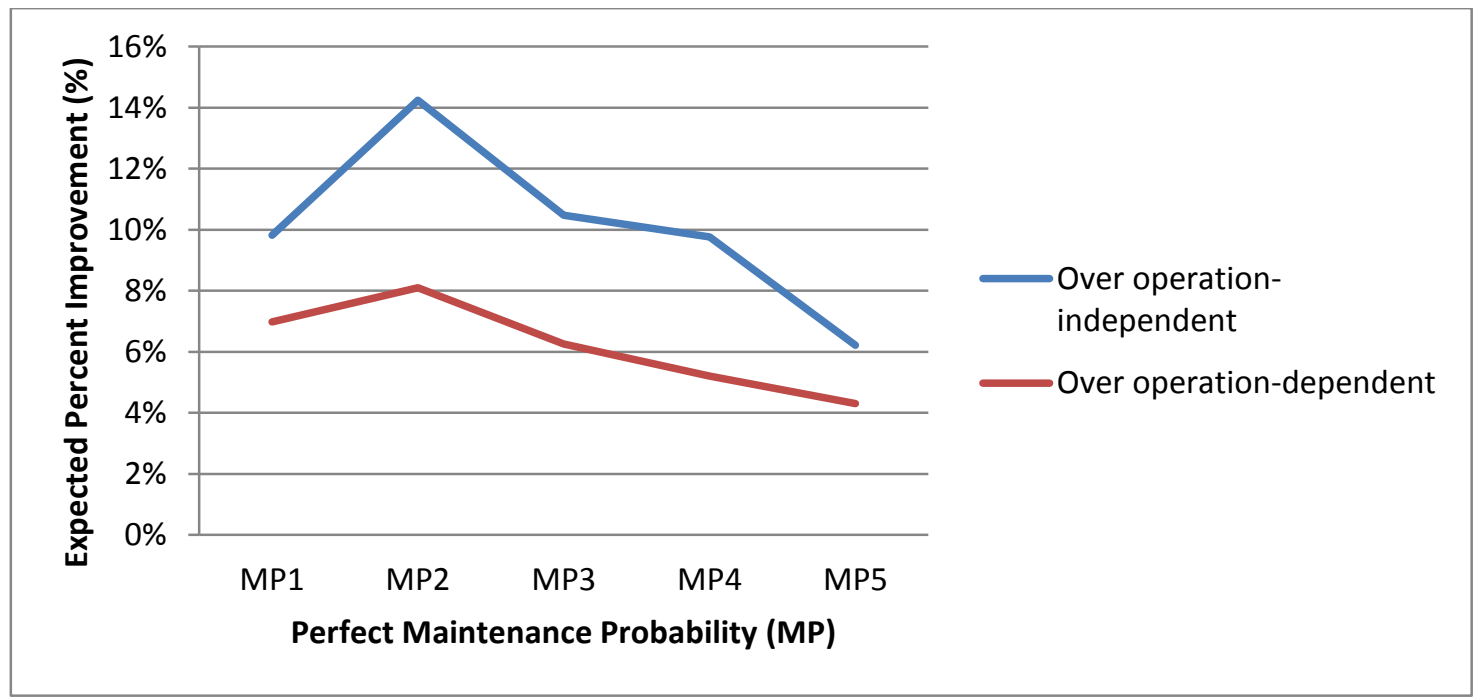

Figure 50. Percent improvement obtained by the integrated decision-making policy over the benchmark policies for different probabilities of perfect maintenance for a system with imperfect state observations 


\subsection{Conclusions}

In this chapter, an integrated decision-making system for maintenance scheduling and product sequencing for a multiple machine/multiple operation manufacturing system with imperfect degradation state observations is presented. Very few researchers have studied the impacts of the environment on the performance of the target system and none have studied the impacts of the target system on the environment. In this study, the decisions of which operations to execute on which equipment are based on the inferred condition of the equipment and hence the condition of the target system impacts the environment. Therefore, to the best of our knowledge, this is the first integrated decisionmaking study considering both the impacts of the environment in the target system and the impact of the target system on the environment.

The integrated decision making policy with imperfect state observations presented in this chapter was applied on an example of a cluster tool model. It was benchmarked against the traditional operation-independent condition based maintenance policy, as well as the operation-dependent condition based maintenance policy both of which assumed imperfect state observations. The performance of the integrated decision-making policy was evaluated under various system parameters such as maintenance probability threshold $(\alpha)$, emission probability matrix, production goals and perfect maintenance probability. The results show that integrated decision-making policy with imperfect state observations outperforms the benchmark policies, in most cases significantly. One instance of the sensitivity analysis for emission probability matrices included the case 
where the relation between the actual degradation states and observation symbols were deterministic. It is shown in Section 5.5.3 that this case is identical to the problem addressed in Chapter 4, i.e. integrated decision-making for a system with perfect degradation state observations. 


\section{Chapter 6}

\section{Summary and Future Work}

The research proposed in this dissertation studies the problems of joint decision making policy for maintenance scheduling and product sequencing in flexible manufacturing systems. The goal is to maximize a customizable profit function with respect to operation-dependent degradation models and production target using various operation mode dependent degradation and yield models for the manufacturing system.

In Chapter 3, which is based on a recent publication [7], a new maintenance decision-making policy based on operation-dependent degradation models and production targets was introduced. The decision-making was done by maximizing a customizable reward function, taking into account rewards of production, costs of maintenance and penalties for unmet production goals. The newly introduced operationdependent decision-making policy was benchmarked against the traditional operationindependent CBM policy, where the maintenance decisions for the equipment are independent of the operations executed in it. The results showed that the operationdependent CBM policy consistently outperformed the benchmark policy in terms of profit. Through the sensitivity analysis, it was observed that higher reactive maintenance costs, unmet production penalty costs, production goals and efficacy of maintenance 
execution lead to more significant relative benefits of the newly proposed maintenance policy over the more traditional operation-independent CBM policy.

In both the newly introduced operation-dependent CBM policy and the traditional operation-independent CBM policy, the sequence in which the products are produced was assumed to be a priori given, which is consistent with the traditional, fragmented approach to maintenance planning and production scheduling. In order to address the problems emanating from this fragmented approach, in Chapter 4, an integrated decisionmaking policy for maintenance scheduling and product sequencing was pursued. The proposed integrated decision-making policy was benchmarked against operationindependent CBM policy and the operation-dependent CBM policy presented in Chapter 3. Through a sensitivity analysis conducted in a simulated environment, the newly proposed methodology was shown to outperform the benchmark policies consistently and often significantly by providing a higher expected profit.

In the manufacturing systems studied in Chapters 3 and 4, the degradation states of the equipment were assumed to be perfectly observable. However, in some highly complex manufacturing environments, the condition of the equipment cannot be directly observed and must be probabilistically inferred from the available sensor readings, i.e. the degradation states are not perfectly observable. Acknowledging this, in Chapter 5, an integrated decision-making policy based on partially observable degradation states was presented for a multiple machine/multiple operation FMS. This new decision-making policy was benchmarked against the traditional operation-independent CBM policy and 
the operation-dependent CBM policy, both of which assumed imperfect state observations. It was shown through a sensitivity analysis that the newly proposed integrated decision-making policy outperformed the benchmark policies consistently even with imperfect state observations. One case of the sensitivity analysis on the emission probability matrix was the situation where the relation between the actual degradation states and observation symbols was deterministic, which is equivalent to having perfect state observations. The results from this case was compared to the results obtained in Chapter 4, confirming this equivalency and showing that the integrated decision-making policy presented in Chapter 5 is a generalization of the policy presented in Chapter 4.

In order to assess the performances of the newly proposed decision-making policies presented in this dissertation, a typical semiconductor manufacturing cluster tool was simulated. Even though the parameters used in these simulations are believed to be representative of the conditions observed in a semiconductor manufacturing fab, an avenue for future work would be obtaining real production and yield parameters from industrial partners and testing the proposed methodologies on a real manufacturing system. Another avenue for future work is to introduce uncertainty to the state transitions describing the Markovian models of degradation processes used in Chapters 3 and 4. Despite some work focusing on the uncertainty of the transition probability matrices in MDPs ([161]-[163]), we are not aware of any previous study on this in the realm of integrated maintenance and production operations decision-making. 
In Chapter 5, the triggering threshold $(\alpha)$ for probabilities of degraded states was regarded as a tunable parameter and a sensitivity analysis was conducted for this parameter. For future work, we believe that the integrated decision-making policy presented in Chapter 5 would greatly benefit from treating $\alpha$ as a variable and optimizing it to find the maximum profit solution. In addition, the emission probability matrices were assumed to be known with certainty. Therefore, another possible extension for the work presented in Chapter 5 would be to introduce uncertainty into the emission probability matrices of HMMs modeling asset degradation.

\subsection{Scientific Contributions}

Each chapter of this doctoral dissertation has certain scientific contributions which resulted in past/foreseen publications. This section will focus on an overview of the scientific contributions that spanned all chapters.

Through this entire doctoral research, the interactions between operations and maintenance in a multi-product/multi-station FMS have been demonstrated and quantified.

A simulation and metaheuristic based optimization framework was devised which enables one to model a manufacturing system as close to reality as possible by allowing the flexibility to use different cost functions, dynamic degradation models which are operating regime specific, various maintenance cost periods and yield models. To the best of our knowledge, this is the first integrated decision-making study in a multiple-station 
manufacturing system that considers both operation-dependent degradation models and a model of the probability of product yield, that is both operation and degradation state dependent. The aforementioned framework was used to evaluate sensitivities and gain an understanding of how effectiveness of integrated decision-making changes with changing parameters.

A new integrated maintenance and operations decision making system was conceived for a FMS with imperfectly observable degradation states. This work is one of the first works in POMDP literature to consider the effects of varying environment on degradation dynamics of the stations. Also, to the best of our knowledge, it is the first work that considers the effects of the condition of the stations on the environment affecting it.

\subsection{Publications}

The publications already produced or anticipated to be produced based on this doctoral research are as follows:

- Celen, M., Djurdjanovic, D., 2012, "Joint Maintenance and Production Operations Decision Making in Flexible Manufacturing Systems," Proceedings of the ASME 2012 International Manufacturing Science and Engineering Conference, Paper ID. MSEC2012-7258. 
- Celen, M., Djurdjanovic, D., 2012, "Operation-Dependent Maintenance Scheduling in Flexible Manufacturing Systems," CIRP Journal of Manufacturing Science and Technology, vol. 5, pp. 296-308. ${ }^{19}$

- Cholette, M. E., Celen, M., Djurdjanovic, D., and Rasberry, J. D., 2013, "Condition Based Monitoring and Operational Decision Making in Semiconductor Manufacturing," IEEE Transactions on Semiconductor Manufacturing, vol. 26, no.4, pp. 454-464.

- Celen, M., Djurdjanovic, D., 2015, "Integrated Maintenance Decision-Making and Product Sequencing in Flexible Manufacturing Systems," ASME Journal of Manufacturing Science and Engineering, vol. 137, no. 4, pp. 1-15.

- Celen, M., Djurdjanovic, D., 2016/2017, "Integrated Maintenance and Operations Decision Making with Imperfect Degradation State Observations," anticipated conference/journal paper based on Chapter 5 .

\footnotetext{
${ }^{19}$ Based on Celen, M., Djurdjanovic, D., 2010, "Operation-Dependent Maintenance Scheduling in Flexible Manufacturing Systems," 44th CIRP International Conference on Manufacturing Systems.

Invited by Prof. Neil Duffie, University of Wisconson - Madison
} 


\section{Chapter 7}

\section{Literature Review of Methods Relevant to Populating Models}

The research presented in this dissertation explores various forms of integrated decision making policies for maintenance scheduling and product sequencing in FMS, with the objective of maximizing a customizable profit function. The performance of these proposed methodologies are tested on a simulated model of a cluster tool. There are several parameters used in these simulations such as cycle time, rewards of production, maintenance time and cost. Such parameters can easily be obtained from historical data in any manufacturing system. On the other hand, identification of the yield models and operation-specific Markov models used in modeling the degradation process of the stations is not straightforward and is out of the scope of this dissertation. However, a review on the most recent methods for operation-dependent degradation and yield model identifications will be presented in this chapter.

\subsection{Review of HMM Estimation}

As mentioned in Chapter 5, in highly complex manufacturing environments, such as the cluster tool simulated in this dissertation, the condition of the equipment cannot be directly observed and can only be probabilistically inferred from the available sensor readings. In addition, the decisions of PM, RM or production decisions are made based 
on uncertain information making this process a POMDP. HMMs are similar to POMDPs in that they have an unobserved underlying state which must be inferred through a series of observations. The main difference being that HMMs are used for monitoring processes without decision making. Therefore, HMM identification methods can be applied directly to learn POMDP parameters but may require more data for training due to the addition of actions [164]. The rest of this section will give a brief overview of HMM identification methods and their applications. However, the main focus will be on identification of operation-specific HMMs as operation-dependent degradation models are utilized in the work presented in this dissertation.

In his tutorial paper [165], Rabiner mentioned that three basic problems must be solved for an HMM model to be useful in real-world applications:

Problem 1 (Evaluation) is the determination of the probability of an observation sequence given an HMM. It is common in classification type tasks and Forward Backward Procedure [165]-[167] can be used to solve Problem 1.

Problem 2 (Decoding) is the determination of the hidden state sequence given an observation sequence and a HMM. The difficulty of this problem lies with the definition of the optimal state sequence [165] as there are several possible optimality criteria. One possible optimality criterion is to choose the hidden states which are individually most likely. Another optimality criterion is to choose the most likely hidden state sequence. Viterbi Algorithm [168] can be employed to find this most likely state sequence. 
Problem 3 (Learning) is the determination of the HMM parameters that maximize the probability of the sequence of observations and is by far the most difficult problem. The well-known Baum-Welch algorithm ([165], [169]) is commonly used to solve this problem. However, Baum-Welch algorithm $(\mathrm{BW})$ and other gradient based search methods can be trapped in local optima and hence metaheuristics methods have been utilized to overcome this drawback of BW ([142], [170]-[172]).

HMMs have found their way into various application areas such as speech recognition ([165], [173], [174]), signature forgery prevention [175], protein structure profiling [176] and equipment condition monitoring ([177]-[183]).

As aforementioned, operation-dependent degradation models have been employed throughout the work presented in this dissertation. However, despite the wide application of HMMs in equipment condition monitoring, identification of operation-specific HMMs have been addressed only recently. In [142], Cholette and Djurdjanovic present a novel data-driven approach for monitoring systems operating under variable conditions. Their method is based on characterizing the degradation process as operation-specific HMMs, where the hidden states represent the unobservable degradation states. The evaluation and decoding problems are solved by using the Forward Procedure and Viterbi Algorithm but with modified definitions of forward and backward variables to account for different operating regimes. For the learning problem, a modified Baum-Welch procedure is presented in detail. In order to avoid the local optima in the presence of operationspecific HMMs, the authors develop a GA-based identification method. Each candidate 
solution in the population was improved by using the modified Baum-Welch procedure and their fitness was determined, where the fitness of any candidate solution was defined as the likelihood of the observation sequence. The solutions with the highest fitness values were then used to generate the next population of candidate solutions. In [184], Cholette also used Tabu Search for local optima avoidance.

Zhang et al. [143] stated not only gradient based search algorithms such as Baum-Welch do not guarantee global optimality, but they also do not readily provide the information on model uncertainty (how close or how far the actual solution is from the maximum likelihood estimate). In addition, model uncertainties accumulate as one attempts to predict probabilities of degradation states further and further ahead and hence could cause the predictions to become useless. In order to address these problems, they proposed a Bayesian estimation scheme for identification of operating regime specific HMM parameters. The capabilities of the proposed Bayesian estimation procedure to identify HMM parameters and the associated parametric uncertainties were first demonstrated via simulations, then on a vast dataset obtained from a PECVD tool operating in a major semiconductor fab.

\subsection{Review of Yield Estimation}

In semiconductor manufacturing fabs, development of in-situ particle monitors has made it possible to assess the level of contamination while the equipment is in use [185]. The use of such monitors also enabled the association of the wafer yields with the 
current condition of the equipment and hence improved maintenance decisions and process control [186]. However, most of the research in this area did not address the impact of yield in product dispatching decisions. Sloan and Shanthikumar [136] was the first study to consider product dispatching in a single-stage system where the product yield was directly linked to the equipment condition. Later Sloan and Shanthikumar [138] extended the work presented in [136] by considering a multi-stage system.

In both [136] and [138], the product yield decreases as the equipment condition worsens. The expected yield values were generated from a beta distribution and sorted. In the work presented in this dissertation, the product yield is operation dependent and decreases with increasing equipment degradation, which is in line with the assumptions in Sloan and Shanthikumar's studies. Therefore, the expected yield values used in the simulations in this work were taken from [138]. 


\section{Appendix}

A.1. Maintenance triggering states for the baseline example in Chapter 3

\begin{tabular}{|c|c|c|c|c|c|}
\cline { 2 - 6 } \multicolumn{1}{c|}{} & \multicolumn{5}{c|}{ OPERATION-INDEPENDENT CBM } \\
\cline { 2 - 6 } \multicolumn{1}{c|}{} & Ch1 & Ch2 & Ch3 & Ch4 & Ch5 \\
\hline Op1 & 3 & 3 & 5 & 5 & 4 \\
Op2 & 3 & 3 & 5 & 3 & 5 \\
Op3 & 5 & 3 & 3 & 5 & 5 \\
Op4 & 3 & 3 & 3 & 5 & 5 \\
Op5 & 3 & 5 & 3 & 5 & 4 \\
Op6 & 3 & 3 & 5 & 5 & 4 \\
Op7 & 3 & 5 & 3 & 5 & 4 \\
Op8 & 3 & 3 & 5 & 3 & 5 \\
Op9 & 3 & 3 & 3 & 5 & 5 \\
Op10 & 5 & 3 & 3 & 5 & 5 \\
Op11 & 5 & 5 & 3 & 5 & 4 \\
Op12 & 5 & 5 & 5 & 3 & 4 \\
Op13 & 5 & 5 & 5 & 3 & 5 \\
\hline
\end{tabular}

\begin{tabular}{|c|c|c|c|c|c|}
\cline { 2 - 6 } \multicolumn{1}{c|}{} & \multicolumn{5}{c|}{ OPERATION-DEPENDENT CBM } \\
\cline { 2 - 6 } & Ch1 & Ch2 & Ch3 3 & Ch4 & Ch5 \\
\hline Op1 & 3 & 3 & 5 & 5 & 4 \\
Op2 & 3 & 3 & 5 & 3 & 5 \\
Op3 & 5 & 2 & 3 & 5 & 5 \\
Op4 & 3 & 3 & 2 & 5 & 5 \\
Op5 & 4 & 5 & 2 & 5 & 4 \\
Op6 & 3 & 4 & 5 & 5 & 3 \\
Op7 & 3 & 5 & 3 & 5 & 4 \\
Op8 & 3 & 3 & 5 & 3 & 5 \\
Op9 & 3 & 4 & 4 & 5 & 5 \\
Op10 & 5 & 5 & 3 & 5 & 5 \\
Op11 & 5 & 5 & 4 & 5 & 3 \\
Op12 & 5 & 5 & 5 & 3 & 3 \\
Op13 & 5 & 5 & 5 & 3 & 5 \\
\hline
\end{tabular}


A.2. Maintenance triggering states for the increasing reactive maintenance costs $\left(c_{r}\right)$ in Chapter 3

\begin{tabular}{|c|c|c|c|c|c|}
\hline & \multicolumn{5}{|c|}{$\begin{array}{c}\text { OPERATION-INDEPENDENT } \\
\text { CBM } \\
\left(c_{r}=100\right)\end{array}$} \\
\hline & Ch1 & Ch2 & Ch3 & Ch4 & Ch5 \\
\hline Op1 & 5 & 3 & 5 & 5 & 3 \\
\hline Op2 & 5 & 3 & 5 & 3 & 5 \\
\hline Op3 & 5 & 3 & 3 & 5 & 5 \\
\hline Op4 & 5 & 3 & 3 & 5 & 5 \\
\hline Op5 & 5 & 5 & 3 & 5 & 3 \\
\hline Op6 & 5 & 3 & 5 & 5 & 3 \\
\hline Op7 & 5 & 5 & 3 & 5 & 3 \\
\hline Op8 & 5 & 3 & 5 & 3 & 5 \\
\hline Op9 & 5 & 3 & 3 & 5 & 5 \\
\hline Op10 & 5 & 3 & 3 & 5 & 5 \\
\hline Op11 & 5 & 5 & 3 & 5 & 3 \\
\hline Op12 & 5 & 5 & 5 & 3 & 3 \\
\hline Op13 & 5 & 5 & 5 & 3 & 5 \\
\hline
\end{tabular}

\begin{tabular}{|c|c|c|c|c|c|}
\cline { 3 - 6 } \multicolumn{1}{c|}{} & \multicolumn{5}{c|}{ OPERATION-INDEPENDENT } \\
CBM \\
\multicolumn{1}{c|}{} & \multicolumn{5}{c|}{$=\mathbf{4 5 0})$} \\
\cline { 2 - 6 } & Ch1 & Ch2 & Ch3 & Ch4 & Ch5 \\
\hline Op1 & 4 & 3 & 5 & 5 & 3 \\
Op2 & 4 & 3 & 5 & 3 & 5 \\
Op3 & 5 & 3 & 2 & 5 & 5 \\
Op4 & 4 & 3 & 2 & 5 & 5 \\
Op5 & 4 & 5 & 2 & 5 & 3 \\
Op6 & 4 & 3 & 5 & 5 & 3 \\
Op7 & 4 & 5 & 2 & 5 & 3 \\
Op8 & 4 & 3 & 5 & 3 & 5 \\
Op9 & 4 & 3 & 2 & 5 & 5 \\
Op10 & 5 & 3 & 2 & 5 & 5 \\
Op11 & 5 & 5 & 2 & 5 & 3 \\
Op12 & 5 & 5 & 5 & 3 & 3 \\
Op13 & 5 & 5 & 5 & 3 & 5 \\
\hline
\end{tabular}

\begin{tabular}{|c|c|c|c|c|c|}
\cline { 2 - 6 } \multicolumn{1}{c|}{} & \multicolumn{5}{c|}{\begin{tabular}{c} 
OPERATION-INDEPENDENT \\
CBM \\
\multicolumn{1}{c|}{} \\
\cline { 2 - 6 } \multicolumn{1}{c|}{$=\mathbf{6 5 0})$}
\end{tabular}} \\
\cline { 2 - 6 } Ch1 & Ch2 & Ch3 & Ch4 & Ch5 \\
\hline Op1 & 3 & 3 & 5 & 5 & 4 \\
Op2 & 3 & 3 & 5 & 3 & 5 \\
Op3 & 5 & 3 & 2 & 5 & 5 \\
Op4 & 3 & 3 & 2 & 5 & 5 \\
Op5 & 3 & 5 & 2 & 5 & 4 \\
Op6 & 3 & 3 & 5 & 5 & 4 \\
Op7 & 3 & 5 & 2 & 5 & 4 \\
Op8 & 3 & 3 & 5 & 3 & 5 \\
Op9 & 3 & 3 & 2 & 5 & 5 \\
Op10 & 5 & 3 & 2 & 5 & 5 \\
Op11 & 5 & 5 & 2 & 5 & 4 \\
Op12 & 5 & 5 & 5 & 3 & 4 \\
Op13 & 5 & 5 & 5 & 3 & 5 \\
\hline
\end{tabular}

\begin{tabular}{|c|c|c|c|c|c|}
\hline & \multicolumn{5}{|c|}{$\begin{array}{c}\text { OPERATION-INDEPENDENT } \\
\text { CBM } \\
\left(c_{r}=850\right) \\
\end{array}$} \\
\hline & Ch1 & Ch2 & Ch3 & Ch4 & Ch5 \\
\hline Op1 & 2 & 3 & 5 & 5 & 4 \\
\hline Op2 & 2 & 3 & 5 & 3 & 5 \\
\hline Op3 & 5 & 3 & 2 & 5 & 5 \\
\hline Op4 & 2 & 3 & 2 & 5 & 5 \\
\hline Op5 & 2 & 5 & 2 & 5 & 4 \\
\hline Op6 & 2 & 3 & 5 & 5 & 4 \\
\hline Op7 & 2 & 5 & 2 & 5 & 4 \\
\hline Op8 & 2 & 3 & 5 & 3 & 5 \\
\hline Op9 & 2 & 3 & 2 & 5 & 5 \\
\hline Op10 & 5 & 3 & 2 & 5 & 5 \\
\hline Op11 & 5 & 5 & 2 & 5 & 4 \\
\hline Op12 & 5 & 5 & 5 & 3 & 4 \\
\hline Op13 & 5 & 5 & 5 & 3 & 5 \\
\hline
\end{tabular}

\begin{tabular}{|c|c|c|c|c|c|}
\cline { 2 - 6 } \multicolumn{1}{c|}{} & \multicolumn{5}{c|}{\begin{tabular}{c} 
OPERATION-DEPENDENT \\
CBM \\
\multicolumn{1}{c|}{} \\
\cline { 2 - 5 } \multicolumn{1}{c|}{$=\mathbf{1 0 0})$}
\end{tabular}} \\
\cline { 2 - 5 } & Ch1 & Ch2 & Ch3 & Ch4 & Ch5 \\
\hline Op1 & 5 & 3 & 5 & 5 & 2 \\
Op2 & 3 & 3 & 5 & 5 & 5 \\
Op3 & 5 & 3 & 3 & 5 & 5 \\
Op4 & 5 & 3 & 2 & 5 & 5 \\
Op5 & 5 & 5 & 2 & 5 & 3 \\
Op6 & 5 & 3 & 5 & 5 & 3 \\
Op7 & 5 & 5 & 3 & 5 & 3 \\
Op8 & 5 & 3 & 5 & 5 & 5 \\
Op9 & 5 & 4 & 5 & 5 & 5 \\
Op10 & 5 & 3 & 3 & 5 & 5 \\
Op11 & 5 & 5 & 5 & 5 & 4 \\
Op12 & 5 & 5 & 5 & 4 & 4 \\
Op13 & 5 & 5 & 5 & 3 & 5 \\
\hline
\end{tabular}

\begin{tabular}{|c|c|c|c|c|c|}
\hline & \multicolumn{5}{|c|}{$\begin{array}{c}\text { OPERATION-DEPENDENT } \\
\text { CBM } \\
\left(c_{r}=450\right)\end{array}$} \\
\hline & Ch1 & Ch2 & Ch3 & Ch4 & Ch5 \\
\hline$\overline{\text { Op1 }}$ & 2 & 2 & 5 & 5 & 2 \\
\hline Op2 & 4 & 3 & 5 & 5 & 5 \\
\hline Op3 & 5 & 3 & 2 & 5 & 5 \\
\hline Op4 & 5 & 3 & 3 & 5 & 5 \\
\hline Op5 & 3 & 5 & 2 & 5 & 3 \\
\hline Op6 & 3 & 3 & 5 & 5 & 5 \\
\hline Op7 & 4 & 5 & 3 & 5 & 3 \\
\hline Op8 & 4 & 5 & 5 & 5 & 5 \\
\hline Op9 & 4 & 4 & 2 & 5 & 5 \\
\hline Op10 & 5 & 3 & 5 & 5 & 5 \\
\hline Op11 & 5 & 5 & 2 & 5 & 3 \\
\hline Op12 & 5 & 5 & 5 & 3 & 3 \\
\hline Op13 & 5 & 5 & 5 & 2 & 5 \\
\hline
\end{tabular}

\begin{tabular}{|c|c|c|c|c|c|}
\hline & \multicolumn{5}{|c|}{$\begin{array}{c}\text { OPERATION-DEPENDENT } \\
\text { CBM } \\
\left(c_{r}=650\right)\end{array}$} \\
\hline & Ch1 & Ch2 & Ch3 & Ch4 & Ch5 \\
\hline Op1 & 2 & 3 & 5 & 5 & 4 \\
\hline Op2 & 4 & 3 & 5 & 3 & 5 \\
\hline Op3 & 5 & 3 & 2 & 5 & 5 \\
\hline Op4 & 3 & 3 & 2 & 5 & 5 \\
\hline Op5 & 3 & 5 & 2 & 5 & 3 \\
\hline Op6 & 3 & 3 & 5 & 5 & 4 \\
\hline Op7 & 3 & 5 & 2 & 5 & 5 \\
\hline Op8 & 3 & 3 & 5 & 3 & 5 \\
\hline Op9 & 4 & 3 & 2 & 5 & 5 \\
\hline Op10 & 5 & 4 & 2 & 5 & 5 \\
\hline Op11 & 5 & 5 & 2 & 5 & 3 \\
\hline Op12 & 5 & 5 & 5 & 3 & 4 \\
\hline Op13 & 5 & 5 & 5 & 3 & 5 \\
\hline
\end{tabular}

\begin{tabular}{|c|c|c|c|c|c|}
\hline & \multicolumn{5}{|c|}{$\begin{array}{c}\text { OPERATION-DEPENDENT } \\
\text { CBM } \\
\left(c_{r}=850\right)\end{array}$} \\
\hline & Ch1 & Ch2 & Ch3 & Ch4 & Ch5 \\
\hline Op1 & 2 & 3 & 5 & 5 & 4 \\
\hline Op2 & 2 & 3 & 5 & 3 & 5 \\
\hline Op3 & 5 & 3 & 2 & 5 & 5 \\
\hline Op4 & 3 & 3 & 2 & 5 & 5 \\
\hline Op5 & 2 & 5 & 2 & 5 & 4 \\
\hline Op6 & 2 & 3 & 5 & 5 & 4 \\
\hline Op7 & 2 & 5 & 2 & 5 & 4 \\
\hline Op8 & 2 & 3 & 5 & 3 & 5 \\
\hline Op9 & 2 & 3 & 2 & 5 & 5 \\
\hline Op10 & 5 & 3 & 2 & 5 & 5 \\
\hline Op11 & 5 & 5 & 2 & 5 & 4 \\
\hline Op12 & 5 & 5 & 5 & 3 & 4 \\
\hline Op13 & 5 & 5 & 5 & 3 & 5 \\
\hline
\end{tabular}


A.3. Maintenance triggering states for the increasing penalty $\operatorname{costs}\left(\mathbf{p}_{w_{j}}\right)$ in Chapter 3

\begin{tabular}{|c|c|c|c|c|c|}
\cline { 2 - 6 } \multicolumn{1}{c|}{} & \multicolumn{5}{c|}{\begin{tabular}{c} 
OPERATION-INDEPENDENT CBM \\
\multicolumn{1}{c|}{$\left(\boldsymbol{p}_{\boldsymbol{w}_{\mathbf{1}}}=\mathbf{1 0}, \boldsymbol{p}_{\boldsymbol{w}_{\mathbf{2}}}=\mathbf{5}, \boldsymbol{p}_{\boldsymbol{w}_{\mathbf{3}}}=\mathbf{2}\right)$}
\end{tabular}} \\
\cline { 2 - 6 } \multicolumn{1}{c|}{} & Ch1 & $\mathbf{C h 2}$ & $\mathbf{C h 3}$ & $\mathbf{C h 4}$ & $\mathbf{C h 5}$ \\
\hline Op1 & 3 & 3 & 5 & 5 & 3 \\
Op2 & 3 & 3 & 5 & 3 & 5 \\
Op3 & 5 & 3 & 2 & 5 & 5 \\
Op4 & 3 & 3 & 2 & 5 & 5 \\
Op5 & 3 & 5 & 2 & 5 & 3 \\
Op6 & 3 & 3 & 5 & 5 & 3 \\
Op7 & 3 & 5 & 2 & 5 & 3 \\
Op8 & 3 & 3 & 5 & 3 & 5 \\
Op9 & 3 & 3 & 2 & 5 & 5 \\
Op10 & 5 & 3 & 2 & 5 & 5 \\
Op11 & 5 & 5 & 2 & 5 & 3 \\
Op12 & 5 & 5 & 5 & 3 & 3 \\
Op13 & 5 & 5 & 5 & 3 & 5 \\
\hline
\end{tabular}

\begin{tabular}{|c|c|c|c|c|c|}
\cline { 2 - 6 } \multicolumn{1}{c|}{} & \multicolumn{5}{c|}{$\begin{array}{c}\text { OPERATION-INDEPENDENT CBM } \\
\left(\boldsymbol{p}_{\boldsymbol{w}_{\mathbf{1}}}=\mathbf{3 0}, \boldsymbol{p}_{\boldsymbol{w}_{\mathbf{2}}}=\mathbf{1 5}, \boldsymbol{p}_{\boldsymbol{w}_{\mathbf{3}}}=\mathbf{6}\right)\end{array}$} \\
\cline { 2 - 6 } \multicolumn{1}{c|}{} & $\mathbf{C h 1}$ & $\mathbf{C h 2}$ & $\mathbf{C h 3}$ & $\mathbf{C h 4}$ & $\mathbf{C h 5}$ \\
\hline Op1 & 3 & 3 & 5 & 5 & 3 \\
Op2 & 3 & 3 & 5 & 3 & 5 \\
Op3 & 5 & 3 & 3 & 5 & 5 \\
Op4 & 3 & 3 & 3 & 5 & 5 \\
Op5 & 3 & 5 & 3 & 5 & 3 \\
Op6 & 3 & 3 & 5 & 5 & 3 \\
Op7 & 3 & 5 & 3 & 5 & 3 \\
Op8 & 3 & 3 & 5 & 3 & 5 \\
Op9 & 3 & 3 & 3 & 5 & 5 \\
Op10 & 5 & 3 & 3 & 5 & 5 \\
Op11 & 5 & 5 & 3 & 5 & 3 \\
Op12 & 5 & 5 & 5 & 3 & 3 \\
Op13 & 5 & 5 & 5 & 3 & 5 \\
\hline
\end{tabular}

\begin{tabular}{|c|c|c|c|c|c|}
\cline { 2 - 6 } \multicolumn{1}{c|}{} & \multicolumn{5}{c|}{\begin{tabular}{c} 
OPERATION-INDEPENDENT CBM \\
\multicolumn{1}{c|}{$\left(\boldsymbol{p}_{\boldsymbol{w}_{\mathbf{1}}}=\mathbf{4 0}, \boldsymbol{p}_{\boldsymbol{w}_{\mathbf{2}}}=\mathbf{2 0}, \boldsymbol{p}_{\boldsymbol{w}_{\mathbf{3}}}=\mathbf{8}\right)$}
\end{tabular}} \\
\cline { 2 - 6 } & Ch1 & $\mathbf{C h 2}$ & $\mathbf{C h 3}$ & $\mathbf{C h} 4$ & $\mathbf{C h 5}$ \\
\hline Op1 & 5 & 3 & 5 & 5 & 3 \\
Op2 & 5 & 3 & 5 & 3 & 5 \\
Op3 & 5 & 3 & 3 & 5 & 5 \\
Op4 & 5 & 3 & 3 & 5 & 5 \\
Op5 & 5 & 5 & 3 & 5 & 3 \\
Op6 & 5 & 3 & 5 & 5 & 3 \\
Op7 & 5 & 5 & 3 & 5 & 3 \\
Op8 & 5 & 3 & 5 & 3 & 5 \\
Op9 & 5 & 3 & 3 & 5 & 5 \\
Op10 & 5 & 3 & 3 & 5 & 5 \\
Op11 & 5 & 5 & 3 & 5 & 3 \\
Op12 & 5 & 5 & 5 & 3 & 3 \\
Op13 & 5 & 5 & 5 & 3 & 5 \\
\hline
\end{tabular}

\begin{tabular}{|c|c|c|c|c|c|}
\cline { 2 - 6 } \multicolumn{1}{c|}{} & \multicolumn{5}{c|}{\begin{tabular}{c} 
OPERATION-DEPENDENT CBM \\
\multicolumn{1}{c|}{$\left(\boldsymbol{p}_{\boldsymbol{w}_{\mathbf{1}}}=\mathbf{1 0}, \boldsymbol{p}_{\boldsymbol{w}_{\mathbf{2}}}=\mathbf{5}, \boldsymbol{p}_{\boldsymbol{w}_{\mathbf{3}}}=\mathbf{2}\right)$}
\end{tabular}} \\
\cline { 2 - 6 } \multicolumn{1}{c|}{} & $\mathbf{C h 1}$ & $\mathbf{C h 2}$ & $\mathbf{C h 3}$ & $\mathbf{C h 4}$ & $\mathbf{C h 5}$ \\
\hline Op1 & 3 & 3 & 5 & 5 & 2 \\
Op2 & 4 & 4 & 5 & 3 & 5 \\
Op3 & 5 & 2 & 2 & 5 & 5 \\
Op4 & 3 & 3 & 3 & 5 & 5 \\
Op5 & 4 & 5 & 2 & 5 & 3 \\
Op6 & 3 & 4 & 5 & 5 & 3 \\
Op7 & 2 & 5 & 2 & 5 & 2 \\
Op8 & 3 & 3 & 5 & 3 & 5 \\
Op9 & 4 & 4 & 5 & 5 & 5 \\
Op10 & 5 & 4 & 5 & 5 & 5 \\
Op11 & 5 & 5 & 4 & 5 & 3 \\
Op12 & 5 & 5 & 5 & 3 & 3 \\
Op13 & 5 & 5 & 5 & 3 & 5 \\
\hline
\end{tabular}

\begin{tabular}{|c|c|c|c|c|c|}
\cline { 2 - 6 } \multicolumn{1}{c|}{} & \multicolumn{5}{c|}{ OPERATION-DEPENDENT CBM } \\
\multicolumn{1}{c|}{$\left(\boldsymbol{p}_{\boldsymbol{w}_{\mathbf{1}}}=\mathbf{3 0}, \boldsymbol{p}_{\boldsymbol{w}_{\mathbf{2}}}=\mathbf{1 5}, \boldsymbol{p}_{\boldsymbol{w}_{\mathbf{3}}}=\mathbf{6}\right)$} \\
\cline { 2 - 6 } \multicolumn{1}{c|}{ Ch1 } & $\mathbf{C h 2} 2$ & $\mathbf{C h 3}$ & $\mathbf{C h} 4$ & $\mathbf{C h 5}$ \\
\hline Op1 & 3 & 3 & 5 & 5 & 3 \\
Op2 & 3 & 3 & 5 & 3 & 5 \\
Op3 & 5 & 3 & 3 & 5 & 5 \\
Op4 & 3 & 3 & 3 & 5 & 5 \\
Op5 & 3 & 5 & 3 & 5 & 3 \\
Op6 & 3 & 3 & 5 & 5 & 3 \\
Op7 & 3 & 5 & 2 & 5 & 3 \\
Op8 & 3 & 3 & 5 & 3 & 5 \\
Op9 & 3 & 3 & 3 & 5 & 5 \\
Op10 & 5 & 3 & 3 & 5 & 5 \\
Op11 & 5 & 5 & 3 & 5 & 3 \\
Op12 & 5 & 5 & 5 & 3 & 3 \\
Op13 & 5 & 5 & 5 & 3 & 5 \\
\hline
\end{tabular}

\begin{tabular}{|c|c|c|c|c|c|}
\cline { 2 - 6 } \multicolumn{1}{c|}{} & \multicolumn{5}{c|}{\begin{tabular}{c} 
OPERATION-DEPENDENT CBM \\
\multicolumn{1}{c|}{$\left(\boldsymbol{p}_{\boldsymbol{w}_{\mathbf{1}}}=\mathbf{4 0}, \boldsymbol{p}_{\boldsymbol{w}_{\mathbf{2}}}=\mathbf{2 0}, \boldsymbol{p}_{\boldsymbol{w}_{\mathbf{3}}}=\mathbf{8}\right)$}
\end{tabular}} \\
\cline { 2 - 6 } & $\mathbf{C h 1}$ & $\mathbf{C h 2}$ & $\mathbf{C h 3}$ & $\mathbf{C h 4}$ & $\mathbf{C h 5}$ \\
\hline Op1 & 5 & 3 & 5 & 5 & 3 \\
Op2 & 5 & 3 & 5 & 3 & 5 \\
Op3 & 5 & 3 & 3 & 5 & 5 \\
Op4 & 5 & 3 & 3 & 5 & 5 \\
Op5 & 5 & 5 & 3 & 5 & 3 \\
Op6 & 5 & 3 & 5 & 5 & 3 \\
Op7 & 5 & 5 & 3 & 5 & 3 \\
Op8 & 5 & 3 & 5 & 3 & 5 \\
Op9 & 2 & 3 & 3 & 5 & 5 \\
Op10 & 5 & 3 & 3 & 5 & 5 \\
Op11 & 5 & 5 & 3 & 5 & 3 \\
Op12 & 5 & 5 & 5 & 3 & 3 \\
Op13 & 5 & 5 & 5 & 3 & 5 \\
\hline
\end{tabular}


A.4. Maintenance triggering states for increasing production goals $\left(\mathrm{N}_{\mathrm{w}_{\mathrm{j}}}\right)$ in Chapter 3

\begin{tabular}{|c|c|c|c|c|c|}
\cline { 2 - 6 } \multicolumn{1}{c|}{} & \multicolumn{5}{c|}{ OPERATION-INDEPENDENT CBM } \\
\multicolumn{1}{c|}{$\left.\boldsymbol{N}_{\boldsymbol{w}_{\mathbf{1}}}=\mathbf{8 5}, \boldsymbol{N}_{\boldsymbol{w}_{\mathbf{2}}}=\mathbf{8 5}, \boldsymbol{N}_{\boldsymbol{w}_{\mathbf{3}}}=\mathbf{8 5}\right)$} \\
\cline { 2 - 6 } \multicolumn{1}{c|}{ Ch1 } & $\mathbf{C h 2}$ & $\mathbf{C h 3}$ & $\mathbf{C h 4}$ & $\mathbf{C h 5}$ \\
\hline Op1 & 3 & 3 & 5 & 5 & 3 \\
Op2 & 3 & 3 & 5 & 3 & 5 \\
Op3 & 5 & 3 & 3 & 5 & 5 \\
Op4 & 3 & 3 & 3 & 5 & 5 \\
Op5 & 3 & 5 & 3 & 5 & 3 \\
Op6 & 3 & 3 & 5 & 5 & 3 \\
Op7 & 3 & 5 & 3 & 5 & 3 \\
Op8 & 3 & 3 & 5 & 3 & 5 \\
Op9 & 3 & 3 & 3 & 5 & 5 \\
Op10 & 5 & 3 & 3 & 5 & 5 \\
Op11 & 5 & 5 & 3 & 5 & 3 \\
Op12 & 5 & 5 & 5 & 3 & 3 \\
Op13 & 5 & 5 & 5 & 3 & 5 \\
\hline
\end{tabular}

\begin{tabular}{|c|c|c|c|c|c|}
\cline { 2 - 6 } \multicolumn{1}{c|}{} & \multicolumn{5}{c|}{ OPERATION-INDEPENDENT CBM } \\
\multicolumn{1}{c|}{$\left(\boldsymbol{N}_{\boldsymbol{w}_{\mathbf{1}}}=\mathbf{9 0}, \boldsymbol{N}_{\boldsymbol{w}_{\mathbf{2}}}=\mathbf{9 0}, \boldsymbol{N}_{\boldsymbol{w}_{\mathbf{3}}}=\mathbf{9 0}\right)$} \\
\cline { 2 - 6 } \multicolumn{1}{c|}{} & $\mathbf{C h 1}$ & $\mathbf{C h 2}$ & $\mathbf{C h 3}$ & $\mathbf{C h 4}$ & $\mathbf{C h 5}$ \\
\hline Op1 & 3 & 3 & 5 & 5 & 5 \\
Op2 & 3 & 3 & 5 & 3 & 5 \\
Op3 & 5 & 3 & 2 & 5 & 5 \\
Op4 & 3 & 3 & 2 & 5 & 5 \\
Op5 & 3 & 5 & 2 & 5 & 5 \\
Op6 & 3 & 3 & 5 & 5 & 5 \\
Op7 & 3 & 5 & 2 & 5 & 5 \\
Op8 & 3 & 3 & 5 & 3 & 5 \\
Op9 & 3 & 3 & 2 & 5 & 5 \\
Op10 & 5 & 3 & 2 & 5 & 5 \\
Op11 & 5 & 5 & 2 & 5 & 5 \\
Op12 & 5 & 5 & 5 & 3 & 5 \\
Op13 & 5 & 5 & 5 & 3 & 5 \\
\hline
\end{tabular}

\begin{tabular}{|c|c|c|c|c|c|}
\cline { 2 - 6 } \multicolumn{1}{c|}{} & \multicolumn{5}{c|}{ OPERATION-INDEPENDENT CBM } \\
\multicolumn{1}{c|}{$\left.\boldsymbol{N}_{\boldsymbol{w}_{\mathbf{1}}}=\mathbf{9 5}, \boldsymbol{N}_{\boldsymbol{w}_{\mathbf{2}}}=\mathbf{9 5}, \boldsymbol{N}_{\boldsymbol{w}_{\mathbf{3}}}=\mathbf{9 5}\right)$} \\
\cline { 2 - 6 } \multicolumn{1}{c|}{ Ch1 } & $\mathbf{C h 2}$ & $\mathbf{C h 3}$ & $\mathbf{C h 4}$ & $\mathbf{C h 5}$ \\
\hline Op1 & 5 & 3 & 5 & 5 & 2 \\
Op2 & 5 & 3 & 5 & 3 & 5 \\
Op3 & 5 & 3 & 2 & 5 & 5 \\
Op4 & 5 & 3 & 2 & 5 & 5 \\
Op5 & 5 & 5 & 2 & 5 & 2 \\
Op6 & 5 & 3 & 5 & 5 & 2 \\
Op7 & 5 & 5 & 2 & 5 & 2 \\
Op8 & 5 & 3 & 5 & 3 & 5 \\
Op9 & 5 & 3 & 2 & 5 & 5 \\
Op10 & 5 & 3 & 2 & 5 & 5 \\
Op11 & 5 & 5 & 2 & 5 & 2 \\
Op12 & 5 & 5 & 5 & 3 & 2 \\
Op13 & 5 & 5 & 5 & 3 & 5 \\
\hline
\end{tabular}

\begin{tabular}{|c|c|c|c|c|c|}
\cline { 2 - 6 } \multicolumn{1}{c|}{} & \multicolumn{5}{c|}{ OPERATION-DEPENDENT CBM } \\
\multicolumn{1}{c|}{$\left(\boldsymbol{N}_{\boldsymbol{w}_{\mathbf{1}}}=\mathbf{8 5}, \boldsymbol{N}_{\boldsymbol{w}_{\mathbf{2}}}=\mathbf{8 5}, \boldsymbol{N}_{\boldsymbol{w}_{\mathbf{3}}}=\mathbf{8 5}\right)$} \\
\cline { 2 - 6 } & Ch1 & $\mathbf{C h 2}$ & $\mathbf{C h 3}$ & $\mathbf{C h 4}$ & $\mathbf{C h 5}$ \\
\hline Op1 & 2 & 3 & 5 & 5 & 3 \\
Op2 & 3 & 3 & 5 & 3 & 5 \\
Op3 & 5 & 3 & 3 & 5 & 5 \\
Op4 & 3 & 3 & 3 & 5 & 5 \\
Op5 & 3 & 5 & 2 & 5 & 3 \\
Op6 & 3 & 5 & 5 & 5 & 3 \\
Op7 & 3 & 5 & 3 & 5 & 3 \\
Op8 & 3 & 3 & 5 & 3 & 5 \\
Op9 & 4 & 2 & 3 & 5 & 5 \\
Op10 & 5 & 3 & 3 & 5 & 5 \\
Op11 & 5 & 5 & 3 & 5 & 3 \\
Op12 & 5 & 5 & 5 & 3 & 3 \\
Op13 & 5 & 5 & 5 & 3 & 5 \\
\hline
\end{tabular}

\begin{tabular}{|c|c|c|c|c|c|}
\cline { 2 - 6 } \multicolumn{1}{c|}{} & \multicolumn{5}{c|}{ OPERATION-DEPENDENT CBM } \\
\multicolumn{1}{c|}{$\left.\boldsymbol{N}_{\boldsymbol{w}_{\mathbf{1}}}=\mathbf{9 0}, \boldsymbol{N}_{\boldsymbol{w}_{\mathbf{2}}}=\mathbf{9 0}, \boldsymbol{N}_{\boldsymbol{w}_{\mathbf{3}}}=\mathbf{9 0}\right)$} \\
\cline { 2 - 6 } & $\mathbf{C h 1}$ & $\mathbf{C h 2}$ & $\mathbf{C h 3}$ & $\mathbf{C h 4}$ & $\mathbf{C h 5}$ \\
\hline Op1 & 3 & 4 & 5 & 5 & 2 \\
Op2 & 3 & 3 & 5 & 3 & 5 \\
Op3 & 5 & 3 & 2 & 5 & 5 \\
Op4 & 3 & 3 & 2 & 5 & 5 \\
Op5 & 5 & 5 & 2 & 5 & 2 \\
Op6 & 3 & 3 & 5 & 5 & 5 \\
Op7 & 3 & 5 & 2 & 5 & 3 \\
Op8 & 3 & 3 & 5 & 4 & 5 \\
Op9 & 3 & 3 & 2 & 5 & 5 \\
Op10 & 5 & 3 & 2 & 5 & 5 \\
Op11 & 5 & 5 & 2 & 5 & 5 \\
Op12 & 5 & 5 & 5 & 3 & 5 \\
Op13 & 5 & 5 & 5 & 3 & 5 \\
\hline
\end{tabular}

\begin{tabular}{|c|c|c|c|c|c|}
\cline { 2 - 6 } \multicolumn{1}{c|}{} & \multicolumn{5}{c|}{ OPERATION-DEPENDENT CBM } \\
\multicolumn{1}{c|}{$\left(\boldsymbol{N}_{\boldsymbol{w}_{\mathbf{1}}}=\mathbf{9 5}, \boldsymbol{N}_{\boldsymbol{w}_{\mathbf{2}}}=\mathbf{9 5}, \boldsymbol{N}_{\boldsymbol{w}_{\mathbf{3}}}=\mathbf{9 5}\right)$}
\end{tabular}


A.5. Maintenance triggering states for the increasing perfect maintenance probabilities (MP) in Chapter 3

\begin{tabular}{|c|c|c|c|c|c|c|c|c|c|c|c|c|c|c|c|c|c|c|c|c|c|c|c|}
\hline & \multicolumn{5}{|c|}{$\begin{array}{c}\text { OPERATION-INDEPENDENT } \\
\text { CBM } \\
\left(M P_{1}\right) \\
\end{array}$} & & \multicolumn{5}{|c|}{$\begin{array}{c}\text { OPERATION-INDEPENDENT } \\
\text { CBM } \\
\left(\mathrm{MP}_{2}\right) \\
\end{array}$} & & \multicolumn{5}{|c|}{$\begin{array}{c}\text { OPERATION-INDEPENDENT } \\
\text { CBM } \\
\left(\mathrm{MP}_{4}\right) \\
\end{array}$} & & \multicolumn{5}{|c|}{$\begin{array}{c}\text { OPERATION-INDEPENDENT } \\
\text { CBM } \\
\left(M P_{5}\right)\end{array}$} \\
\hline & Ch1 & Ch2 & Ch3 & Ch4 & Ch5 & & Ch1 & Ch2 & \begin{tabular}{|l|} 
Ch3 \\
\end{tabular} & Ch4 & Ch5 & & Ch1 & $\mathrm{Ch2}$ & Ch3 & Ch4 & Ch5 & & Ch1 & Ch2 & Ch3 & Ch4 & Ch5 \\
\hline Op1 & 4 & 2 & 5 & 5 & 3 & Op1 & 5 & 3 & 5 & 5 & 2 & Op1 & 3 & 3 & 5 & 5 & 3 & Op1 & 3 & 3 & 5 & 5 & 3 \\
\hline Op2 & 4 & 2 & 5 & 2 & 5 & Op2 & 5 & 3 & 5 & 3 & 5 & Op2 & 3 & 3 & 5 & 3 & 5 & Op2 & 3 & 3 & 5 & 3 & 5 \\
\hline Op3 & 5 & 2 & 2 & 5 & 5 & Op3 & 5 & 3 & 2 & 5 & 5 & Op3 & 5 & 3 & 3 & 5 & 5 & Op3 & 5 & 3 & 3 & 5 & 5 \\
\hline Op4 & 4 & 2 & 2 & 5 & 5 & Op4 & 5 & 3 & 2 & 5 & 5 & Op4 & 3 & 3 & 3 & 5 & 5 & Op4 & 3 & 3 & 3 & 5 & 5 \\
\hline Op5 & 4 & 5 & 2 & 5 & 3 & Op5 & 5 & 5 & 2 & 5 & 2 & Op5 & 3 & 5 & 3 & 5 & 3 & Op5 & 3 & 5 & 3 & 5 & 3 \\
\hline Op6 & 4 & 2 & 5 & 5 & 3 & Op6 & 5 & 3 & 5 & 5 & 2 & Op6 & 3 & 3 & 5 & 5 & 3 & Op6 & 3 & 3 & 5 & 5 & 3 \\
\hline Op7 & 4 & 5 & 2 & 5 & 3 & Op7 & 5 & 5 & 2 & 5 & 2 & Op7 & 3 & 5 & 3 & 5 & 3 & Op7 & 3 & 5 & 3 & 5 & 3 \\
\hline Op8 & 4 & 2 & 5 & 2 & 5 & Op8 & 5 & 3 & 5 & 3 & 5 & Op8 & 3 & 3 & 5 & 3 & 5 & Op8 & 3 & 3 & 5 & 3 & 5 \\
\hline Op9 & 4 & 2 & 2 & 5 & 5 & Op9 & 5 & 3 & 2 & 5 & 5 & Op9 & 3 & 3 & 3 & 5 & 5 & Op9 & 3 & 3 & 3 & 5 & 5 \\
\hline Op10 & 5 & 2 & 2 & 5 & 5 & Op10 & 5 & 3 & 2 & 5 & 5 & Op10 & 5 & 3 & 3 & 5 & 5 & Op10 & 5 & 3 & 3 & 5 & 5 \\
\hline Op11 & 5 & 5 & 2 & 5 & 3 & Op11 & 5 & 5 & 2 & 5 & 2 & Op11 & 5 & 5 & 3 & 5 & 3 & Op11 & 5 & 5 & 3 & 5 & 3 \\
\hline Op12 & 5 & 5 & 5 & 2 & 3 & Op12 & 5 & 5 & 5 & 3 & 2 & Op12 & 5 & 5 & 5 & 3 & 3 & Op12 & 5 & 5 & 5 & 3 & 3 \\
\hline Op13 & 5 & 5 & 5 & 2 & 5 & Op13 & 5 & 5 & 5 & 3 & 5 & Op13 & 5 & 5 & 5 & 3 & 5 & Op13 & 5 & 5 & 5 & 3 & 5 \\
\hline
\end{tabular}

\begin{tabular}{|c|c|c|c|c|c|c|c|c|c|c|c|c|c|c|c|c|c|c|c|c|c|c|c|}
\hline & \multicolumn{5}{|c|}{$\begin{array}{c}\text { OPERATION-DEPENDENT } \\
\text { CBM } \\
\left(M P_{1}\right)\end{array}$} & & \multicolumn{5}{|c|}{$\begin{array}{c}\text { OPERATION-DEPENDENT } \\
\text { CBM } \\
\left(M_{2}\right) \\
\end{array}$} & & \multicolumn{5}{|c|}{$\begin{array}{c}\text { OPERATION-DEPENDENT } \\
\text { CBM } \\
\left(M_{4}\right)\end{array}$} & & \multicolumn{5}{|c|}{$\begin{array}{c}\text { OPERATION-DEPENDENT } \\
\text { CBM } \\
\left(M P_{5}\right)\end{array}$} \\
\hline & Ch1 & Ch2 & Ch3 & Ch4 & Ch5 & & Ch1 & Ch2 & Ch3 & Ch4 & Ch5 & & Ch1 & Ch2 & Ch3 & Ch4 & Ch5 & & Ch1 & Ch2 & Ch3 & Ch4 & Ch5 \\
\hline Op1 & 4 & 2 & 5 & 5 & 3 & Op1 & 5 & 3 & 5 & 5 & 2 & Op1 & 3 & 3 & 5 & 5 & 3 & Op1 & 3 & 3 & 5 & 5 & 3 \\
\hline Op2 & 4 & 4 & 5 & 5 & 5 & Op2 & 4 & 3 & 5 & 3 & 5 & Op2 & 3 & 3 & 5 & 5 & 5 & Op2 & 3 & 3 & 5 & 3 & 5 \\
\hline Op3 & 5 & 3 & 2 & 5 & 5 & Op3 & 5 & 3 & 2 & 5 & 5 & Op3 & 5 & 3 & 3 & 5 & 5 & Op3 & 5 & 3 & 3 & 5 & 5 \\
\hline Op4 & 4 & 2 & 2 & 5 & 5 & Op4 & 5 & 3 & 2 & 5 & 5 & Op4 & 3 & 3 & 4 & 5 & 5 & Op4 & 3 & 3 & 3 & 5 & 5 \\
\hline Op5 & 4 & 5 & 2 & 5 & 3 & Op5 & 5 & 5 & 2 & 5 & 2 & Op5 & 5 & 5 & 3 & 5 & 2 & Op5 & 3 & 5 & 3 & 5 & 3 \\
\hline Op6 & 2 & 3 & 5 & 5 & 3 & Op6 & 5 & 3 & 5 & 5 & 2 & Op6 & 3 & 5 & 5 & 5 & 3 & Op6 & 5 & 3 & 5 & 5 & 3 \\
\hline Op7 & 2 & 5 & 2 & 5 & 3 & Op7 & 5 & 5 & 2 & 5 & 2 & Op7 & 3 & 5 & 3 & 5 & 3 & Op7 & 3 & 5 & 3 & 5 & 3 \\
\hline Op8 & 4 & 2 & 5 & 4 & 5 & Op8 & 5 & 3 & 5 & 3 & 5 & Op8 & 3 & 3 & 5 & 3 & 5 & Op8 & 3 & 3 & 5 & 3 & 5 \\
\hline Op9 & 5 & 5 & 3 & 5 & 5 & Op9 & 5 & 3 & 2 & 5 & 5 & Op9 & 3 & 3 & 3 & 5 & 5 & Op9 & 3 & 3 & 3 & 5 & 5 \\
\hline Op10 & 5 & 3 & 2 & 5 & 5 & Op10 & 5 & 3 & 2 & 5 & 5 & Op10 & 5 & 5 & 3 & 5 & 5 & Op10 & 5 & 3 & 3 & 5 & 5 \\
\hline Op11 & 5 & 5 & 2 & 5 & 3 & Op11 & 5 & 5 & 2 & 5 & 2 & Op11 & 5 & 5 & 4 & 5 & 3 & Op11 & 5 & 5 & 3 & 5 & 3 \\
\hline Op12 & 5 & 5 & 5 & 2 & 3 & Op12 & 5 & 5 & 5 & 3 & 2 & Op12 & 5 & 5 & 5 & 3 & 3 & Op12 & 5 & 5 & 5 & 3 & 3 \\
\hline Op13 & 5 & 5 & 5 & 2 & 5 & Op13 & 5 & 5 & 5 & 3 & 5 & Op13 & 5 & 5 & 5 & 3 & 5 & Op13 & 5 & 5 & 5 & 3 & 5 \\
\hline
\end{tabular}




\section{Bibliography}

[1] H. Wang, "A survey of maintenance policies of deteriorating systems," Eur. J. Oper. Res., vol. 139, no. 3, pp. 469-489, Jun. 2002.

[2] S. a. Spiewak, R. Duggirala, and K. Barnett, "Predictive Monitoring and Control of the Cold Extrusion Process," CIRP Ann. - Manuf. Technol., vol. 49, no. 1, pp. 383-386, Jan. 2000.

[3] S. Lee, D. Djurdjanovic, and J. Ni, "Optimal Condition-Based Maintenance Decision Making for a Cluster Tool," in TECHCON, 2007, pp. 1-4.

[4] M. Marseguerra, E. Zio, and L. Podofillini, "Condition-based maintenance optimization by means of genetic algorithms and Monte Carlo simulation," Reliab. Eng. Syst. Saf., vol. 77, no. 2, pp. 151-166, Aug. 2002.

[5] J. Zhou, D. Djurdjanovic, J. Ivy, and J. Ni, "Integrated reconfiguration and agebased preventive maintenance decision making," IIE Trans., vol. 39, no. 12, pp. 1085-1102, Oct. 2007.

[6] Z. Yang, D. Djurdjanovic, and J. Ni, "Maintenance scheduling for a manufacturing system of machines with adjustable throughput," IIE Trans., vol. 39, pp. 11111125, 2007.

[7] M. Celen and D. Djurdjanovic, "Operation-Dependent Maintenance Scheduling in Flexible Manufacturing Systems," CIRP J. Manuf. Sci. Technol., vol. 5, pp. 296308, 2012.

[8] M. Celen and D. Djurdjanovic, "Integrated Maintenance Decision-Making and Product Sequencing in Flexible Manufacturing Systems," ASME J. Manuf. Sci. Eng., vol. 137, no. 4, pp. 1-15, 2015.

[9] M. E. Cholette, M. Celen, D. Djurdjanovic, and J. D. Rasberry, "Condition Monitoring and Operational Decision Making in Semiconductor Manufacturing," IEEE Trans. Semicond. Manuf., vol. 26, no. 4, pp. 454-464, 2013.

[10] T. Chitra, "Life based maintenance policy for minimum cost," in Annual Reliability and Maintainability Symposium, 2003, pp. 470-474.

[11] E. Iakovou, C. Ip, and C. Koulamas, "Throughput-dependent periodic maintenance policiesfor general production units," Ann. Oper. Res., vol. 91, pp. 41-47, 1999.

[12] Y.-T. Tsai, K.-S. Wang, and H.-Y. Teng, "Optimizing preventive maintenance for mechanical components using genetic algorithms," Reliab. Eng. Syst. Saf., vol. 74, no. 1, pp. 89-97, Oct. 2001.

[13] N. Rezg, A. Chelbi, and X. Xie, "Modeling and optimizing a joint inventory control and preventive maintenance strategy for a randomly failing production unit: Analytical and simulation approaches," Int. J. Comput. Integr. Manuf., vol. 18, no. 2-3, pp. 225-235, Mar. 2005. 
[14] J.-P. Kenné, a. Gharbi, and M. Beit, "Age-dependent production planning and maintenance strategies in unreliable manufacturing systems with lost sale," Eur. J. Oper. Res., vol. 178, no. 2, pp. 408-420, Apr. 2007.

[15] N. Rezg, S. Dellagi, and a. Chelbi, "Joint optimal inventory control and preventive maintenance policy," Int. J. Prod. Res., vol. 46, no. 19, pp. 5349-5365, Oct. 2008.

[16] K. A. H. Kobbacy, N. C. Proudlove, and M. A. Harper, "Towards an intelligent maintenance optimization system,” J. Oper. Res. Soc., vol. 46, no. 7, pp. 831-853, 1995.

[17] R. Barlow and L. Hunter, "Optimum preventive maintenance policies," Oper. Res., vol. 8, no. 1, pp. 90-100, 1960.

[18] Y. Sherif and M. Smith, "Optimal maintenance models for systems subject to failure-a review," Nav. Res. Logist. Q., vol. 28, no. 1, pp. 47-74, 1981.

[19] C. Valdez- $\square$ Flores and R. Feldman, "A survey of preventive maintenance models for stochastically deteriorating single $\square$ unit systems," Nav. Res. Logist., vol. 36, no. 4, pp. 419-446, 1989.

[20] S. Zeng, "Discussion on maintenance strategy, policy and corresponding maintenance systems in manufacturing," Reliab. Eng. Syst. Saf., vol. 55, pp. 151$162,1997$.

[21] E. Fernandez, M. Fu, and S. Marcus, "Survey of Current Best Practices in Preventive Maintenance Scheduling in Semiconductor Manufacturing," SRC Deliv. Rep. Task ID 877.001, 2002.

[22] A. Garg and S. G. Deshmukh, "Maintenance management: literature review and directions," J. Qual. Maint. Eng., vol. 12, no. 3, pp. 205-238, 2006.

[23] H. Pham, A. Suprasad, and R. Misra, "Availability and mean life time prediction of multistage degraded system with partial repairs," Reliab. Eng. Syst. Saf., vol. 56, pp. 169-173, 1997.

[24] H. Pham and H. Wang, "Imperfect maintenance," Eur. J. Oper. Res., vol. 94, no. 3, pp. 425-438, Nov. 1996.

[25] V. Mijailovic, "Probabilistic method for planning of maintenance activities of substation components," Electr. Power Syst. Res., vol. 64, pp. 53-58, 2003.

[26] V. Legat, A. Žaludová, V. Cervenka, and V. Jurca, "Contribution to optimization of preventive replacement," Reliab. Eng. Syst. Saf., vol. 51, pp. 259-266, 1996.

[27] R. V. Canfield, "Cost Optimization of Periodic Preventive Maintenance," IEEE Trans. Reliab., vol. R-35, no. 1, pp. 78-81, 1986.

[28] A.-S. Charles, I.-R. Floru, C. Azzaro-Pantel, L. Pibouleau, and S. Domenech, "Optimization of preventive maintenance strategies in a multipurpose batch plant: application to semiconductor manufacturing," Comput. Chem. Eng., vol. 27, no. 4, pp. 449-467, Apr. 2003. 
[29] T. Nakagawa, "Optimum Policies When Preventive Maintenance is Imperfect," IEEE Trans. Reliab., vol. R-28, no. 4, pp. 331-332, Oct. 1979.

[30] D. H. Park, G. M. Jung, and J. K. Yum, "Cost minimization for periodic maintenance policy of a system subject to slow degradation," Reliab. Eng. Syst. Saf., vol. 68, no. 2, pp. 105-112, May 2000.

[31] D. Chaudhuri and K. Sahu, "Preventive Maintenance Interval for Optimal Reliability of Deteriorating System," IEEE Trans. Reliab., vol. R-26, no. 5, pp. 371-372, 1977.

[32] H. Wang and H. Pham, "Some maintenance models and availability withimperfect maintenance in production systems," Ann. Oper. Res., vol. 91, pp. 305-318, 1999.

[33] F. Badia, M. Berrade, and C. Campos, "Optimal inspection and preventive maintenance of units with revealed and unrevealed failures," Reliab. Eng. Syst. ..., vol. 78, pp. 157-163, 2002.

[34] P. Bruns, "Optimal maintenance strategies for systems with partial repair options and without assuming bounded costs," Eur. J. Oper. Res., vol. 139, no. 1, pp. 146165, May 2002.

[35] K. Linderman, K. E. McKone-Sweet, and J. C. Anderson, "An integrated systems approach to process control and maintenance," Eur. J. Oper. Res., vol. 164, no. 2, pp. 324-340, Jul. 2005.

[36] T. Nakagawa and D. Murthy, "Optimal replacement policies for a two-unit system with failure interactions," RAIRO. Rech. opérationnelle, vol. 27, no. 4, pp. 427438, 1993.

[37] I. Gertsbakh, "Optimal group preventive maintenance of a system with observable state parameter," Adv. Appl. Probab., vol. 16, no. 4, pp. 923-925, 1984.

[38] S. Sheu and J. Jhang, "Theory and Methodology: A generalized group maintenance policy," Eur. J. Oper. Res., vol. 96, pp. 232-247, 1996.

[39] J. Dagpunar, "A maintenance model with opportunities and interrupt replacement options,” J. Oper. Res. Soc., vol. 47, no. 11, pp. 1406-1409, 1996.

[40] R. Ahmad and S. Kamaruddin, "An overview of time-based and condition-based maintenance in industrial application," Comput. Ind. Eng., vol. 63, no. 1, pp. 135149, 2012.

[41] A. H. Christer and W. Wang, "A simple condition monitoring model for a direct monitoring process," Eur. J. Oper. Res., vol. 82, no. 2, pp. 258-269, Apr. 1995.

[42] J. a. M. Hontelez, H. H. Burger, and D. J. D. Wijnmalen, "Optimum conditionbased maintenance policies for deteriorating systems with partial information," Reliab. Eng. Syst. Saf., vol. 51, no. 3, pp. 267-274, Mar. 1996.

[43] F. Barbera, H. Schneider, and P. Kelle, "A condition based maintenance model with exponential failures and fixed inspection intervals," J. Oper. Res. Soc., vol. 47, no. 8, pp. 1037-1045, 1996. 
[44] T. Aven, "Condition based replacement policies - a counting process approach," Reliab. Eng. Syst. Saf., vol. 51, no. 3, pp. 275-281, Mar. 1996.

[45] F. Cadini, E. Zio, and D. Avram, "Model-based Monte Carlo state estimation for condition-based component replacement," Reliab. Eng. Syst. Saf., vol. 94, no. 3, pp. 752-758, Mar. 2009.

[46] L. Dieulle, C. Berenguer, A. Grall, and M. Roussignol, "Continuous-time predictive-maintenance scheduling for a deteriorating system," in Annual Reliability and Maintainability Symposium, 2001, pp. 150-155.

[47] A. Grall, C. Bérenguer, and L. Dieulle, "A condition-based maintenance policy for stochastically deteriorating systems," Reliab. Eng. Syst. Saf., vol. 76, no. 2, pp. 167-180, May 2002.

[48] L. Dieulle, C. Bérenguer, a Grall, and M. Roussignol, "Sequential condition-based maintenance scheduling for a deteriorating system," Eur. J. Oper. Res., vol. 150, no. 2, pp. 451-461, Oct. 2003.

[49] R. Ahmadi and M. Newby, "Maintenance scheduling of a manufacturing system subject to deterioration," Reliab. Eng. Syst. Saf., vol. 96, no. 10, pp. 1411-1420, Oct. 2011.

[50] V. a. Kopnov, "Optimal degradation processes control by two-level policies," Reliab. Eng. Syst. Saf., vol. 66, no. 1, pp. 1-11, Oct. 1999.

[51] J. Barata, C. G. Soares, M. Marseguerra, and E. Zio, "Simulation modelling of repairable multi-component deteriorating systems for 'on condition' maintenance optimisation," Reliab. Eng. Syst. Saf., vol. 76, no. 3, pp. 255-264, Jun. 2002.

[52] X. Zhou, L. Xi, and J. Lee, "A dynamic opportunistic maintenance policy for continuously monitored systems," J. Qual. Maint. Eng., vol. 12, no. 3, pp. 294305, 2006.

[53] B. Saassouh, L. Dieulle, and a. Grall, "Online maintenance policy for a deteriorating system with random change of mode," Reliab. Eng. Syst. Saf., vol. 92, no. 12, pp. 1677-1685, Dec. 2007.

[54] W. Liao, E. Pan, and L. Xi, "Preventive maintenance scheduling for repairable system with deterioration," J. Intell. Manuf., vol. 21, no. 6, pp. 875-884, Apr. 2009.

[55] W. Wang and A. Christer, "Towards a general condition based maintenance model for a stochastic dynamic system," J. Oper. Res. Soc., vol. 51, no. 2, pp. 145-155, 2000 .

[56] V. Makis and X. Jiang, "Optimal Replacement Under Partial Observations," Math. Oper. Res., vol. 28, no. 2, pp. 382-394, May 2003.

[57] L. M. Maillart, "Maintenance policies for systems with condition monitoring and obvious failures," IIE Trans., vol. 38, no. 6, pp. 463-475, Jul. 2006.

[58] A. N. Njike, R. Pellerin, and J. P. Kenne, "Maintenance/production planning with 
interactive feedback of product quality," J. Qual. Maint. Eng., vol. 17, no. 3, pp. 281-298, 2011.

[59] A. Prajapati, J. Bechtel, and S. Ganesan, "Condition based maintenance: a survey," J. Qual. Maint. Eng., vol. 18, no. 4, pp. 384-400, 2012.

[60] J.-H. Shin and H.-B. Jun, "On condition based maintenance policy," J. Comput. Des. Eng., vol. 2, no. 2, pp. 119-127, 2015.

[61] A. K. S. Jardine, D. Lin, and D. Banjevic, "A review on machinery diagnostics and prognostics implementing condition-based maintenance," Mech. Syst. Signal Process., vol. 20, pp. 1483-1510, 2006.

[62] R. C. M. Yam, P. W. Tse, L. Li, and P. Tu, "Intelligent Predictive Decision Support System for Condition-Based Maintenance," Int. J. Adv. Manuf. Technol., vol. 17, no. 5, pp. 383-391, Feb. 2001.

[63] S. Wu, N. Gebraeel, M. A. Lawley, and Y. Yih, “A Neural Network Integrated Decision Support System for Condition-Based Optimal Predictive Maintenance Policy," IEEE Trans. Syst. Man. Cybern., vol. 37, no. 2, pp. 226-236, 2007.

[64] K. A. Kaiser and N. Z. Gebraeel, "Predictive Maintenance Management Using Sensor-Based Degradation Models," IEEE Trans. Syst. Man. Cybern., vol. 39, no. 4, pp. 840-849, 2009.

[65] A. H. Elwany and N. Z. Gebraeel, "Sensor-driven prognostic models for equipment replacement and spare parts inventory," IIE Trans., vol. 40, no. 7, pp. 629-639, 2008.

[66] M. You, F. Liu, W. Wang, and G. Meng, "Statistically Planned and Individually Improved Predictive Maintenance Management for Continuously Monitored Degrading Systems," IEEE Trans. Reliab., vol. 59, no. 4, pp. 744-753, 2010.

[67] M. C. Garcia, M. A. Sanz-Bobi, and J. del Pico, "SIMAP : Intelligent System for Predictive Maintenance Application to the health condition monitoring of a windturbine gearbox," Comput. Ind., vol. 57, pp. 552-568, 2006.

[68] S. Lu, Y.-C. Tu, and H. Lu, "Predictive condition-based maintenance for continuously deteriorating systems," Qual. Reliab. Eng. Int., vol. 23, pp. 71-81, 2007.

[69] G. Curcurù, G. Galante, and A. Lombardo, "A predictive maintenance policy with imperfect monitoring," Reliab. Eng. Syst. Saf., vol. 95, pp. 989-997, 2010.

[70] M.-Y. You, L. Li, G. Meng, and J. Ni, "Cost-Effective Updated Sequential Predictive Maintenance Policy for Continuously Monitored Degrading Systems," IEEE Trans. Autom. Sci. Eng., vol. 7, no. 2, pp. 257-265, 2010.

[71] Z. (Max) Yang, D. Djurdjanovic, and J. Ni, "Maintenance scheduling in manufacturing systems based on predicted machine degradation," J. Intell. Manuf., vol. 19, no. 1, pp. 87-98, Jul. 2008.

[72] S. M. Pandit and S.-M. Wu, Time series and system analysis with application. 
Malabar, FL: Krieger Publishing Co, 1993.

[73] G. Yu, H. Qiu, D. Djurdjanovic, and J. Lee, "Feature signature prediction of a boring process using neural network modeling with confidence bounds," Int. J. Adv. Manuf. Technol., vol. 30, pp. 614-621, 2006.

[74] D. Djurdjanovic, J. Lee, and J. Ni, "Watchdog Agent — an infotronics-based prognostics approach for product performance degradation assessment and prediction," Adv. Eng. Informatics, vol. 17, pp. 109-125, 2003.

[75] R. Dekker, "Applications of maintenance optimization models: a review and analysis,” Reliab. Eng. Syst. Saf., vol. 51, no. 3, pp. 229-240, Mar. 1996.

[76] D. Chen and K. S. Trivedi, "Closed-form analytical results for condition-based maintenance," Reliab. Eng. Syst. Saf., vol. 76, no. 1, pp. 43-51, Apr. 2002.

[77] Z. Yamayee, "A computationally efficient optimal maintenance scheduling method," IEEE Trans. Power Appar. Syst., vol. PAS-102, no. 2, pp. 330-338, 1983.

[78] H. Zurn and V. Quintana, "Generator maintenance scheduling via successive approximations dynamic programming," IEEE Trans. Power Appar. Syst., vol. PAS-94, no. 2, pp. 665-671, 1975.

[79] J. Dopazo and H. Merrill, "Optimal generator maintenance scheduling using integer programming," IEEE Trans. Power Appar. Syst., vol. PAS-94, no. 5, pp. 1537-1545, 1975.

[80] G. T. Egan, T. S. Dillon, and K. Morsztyn, "An Experimental Method of Determination of Optimal Maintenance Schedules in Power Systems Using the Branch-and-Bound Technique," IEEE Trans. Syst. Man. Cybern., vol. 6, no. 8, pp. 538-547, 1976.

[81] G. Tagaras, "An integrated cost model for the joint optimization of process control and maintenance," J. Oper. Res. Soc., vol. 39, no. 8, pp. 757-766, 1988.

[82] T. Das and S. Sarkar, "Optimal preventive maintenance in a production inventory system,” IIE Trans., vol. 31, pp. 537-551, 1999.

[83] H. Kim, K. Nara, and M. Gen, "A method for maintenance scheduling using GA combined with SA,” Comput. Ind. Eng., vol. 27, no. 94, pp. 477-480, 1994.

[84] J. Holland, Adaptation in natural and artificial systems. Cambridge, MA: MIT Press, 1992.

[85] T. Back, Evolutionary algorithms in theory and practice. USA: Oxford University Press, 1996.

[86] A. Munõz, S. Martorell, and V. Serradell, "Genetic algorithms in optimizing surveillance and maintenance of components," Reliab. Eng. Syst. Saf., vol. 57, pp. 107-120, 1997.

[87] G. Cavory, R. Dupas, and G. Goncalves, "A genetic approach to the scheduling of 
preventive maintenance tasks on a single product manufacturing production line," Int. J. Prod. Econ., vol. 74, no. 1-3, pp. 135-146, Dec. 2001.

[88] M. Marseguerra and E. Zio, "Optimizing maintenance and repair policies via a combination of genetic algorithms and Monte Carlo simulation," Reliab. Eng. Syst. Saf., vol. 68, no. 1, pp. 69-83, Apr. 2000.

[89] M. A. Ilgin and S. Tunali, "Joint optimization of spare parts inventory and maintenance policies using genetic algorithms," Int. J. Adv. Manuf. Technol., vol. 34, no. 5-6, pp. 594-604, May 2007.

[90] L. Wang, J. Chu, and W. Mao, "A condition-based replacement and spare provisioning policy for deteriorating systems with uncertain deterioration to failure," Eur. J. Oper. Res., vol. 194, no. 1, pp. 184-205, Apr. 2009.

[91] M. Machani and M. Nourelafath, "A genetic algorithm for integrated production and preventive maintenance planning in multi-state systems," in International Conference of Modeling and Simulation, 2010.

[92] Z. Yang, Q. Chang, D. Djurdjanovic, J. Ni, and J. Lee, "Maintenance Priority Assignment Utilizing On-line Production Information," J. Manuf. Sci. Eng., vol. 129, no. 2, pp. 435-446, 2007.

[93] F. Camci, "System Maintenance Scheduling With Prognostics Information Using Genetic Algorithm," IEEE Trans. Reliab., vol. 58, no. 3, pp. 539-552, Sep. 2009.

[94] S. Deris, S. Omatu, H. Ohta, L. C. Shaharudin Kutar, and P. Abd Samat, "Ship maintenance scheduling by genetic algorithm and constraint-based reasoning," Eur. J. Oper. Res., vol. 112, no. 3, pp. 489-502, Feb. 1999.

[95] J. Gonçalves and J. De Almeida, "A hybrid genetic algorithm for assembly line balancing," J. Heuristics, vol. 8, pp. 629-642, 2002.

[96] S. H. Chung, F. T. S. Chan, and H. K. Chan, "A modified genetic algorithm approach for scheduling of perfect maintenance in distributed production scheduling," Eng. Appl. Artif. Intell., vol. 22, no. 7, pp. 1005-1014, Oct. 2009.

[97] N. Metropolis, A. W. Rosenbluth, M. N. Rosenbluth, A. H. Teller, and E. Teller, "Equation of State Calculations by Fast Computing Machines," J. Chem. Phys., vol. 21, no. 6, pp. 1087-1092, 1953.

[98] S. Kirkpatrick, C. D. Gelatt, and M. P. Vecchi, "Optimization by simulated annealing.," Science, vol. 220, no. 4598, pp. 671-680, May 1983.

[99] V. Cerný, "Thermodynamical approach to the traveling salesman problem: An efficient simulation algorithm," J. Optim. Theory Appl., vol. 45, no. 1, pp. 41-51, 1985.

[100] L. Ingber, "Simulated annealing: Practice versus theory," Math. Comput. Model., vol. 18, no. 11, pp. 29-57, 1993.

[101] T. Satoh and K. Nara, "Maintenance scheduling by using simulated annealing method," IEEE Trans. Power Syst., pp. 850-857, 1991. 
[102] J. T. Saraiva, M. L. Pereira, V. T. Mendes, and J. C. Sousa, "A Simulated Annealing based approach to solve the generator maintenance scheduling problem," Electr. Power Syst. Res., vol. 81, no. 7, pp. 1283-1291, Jul. 2011.

[103] K. P. Dahal, G. M. Burt, J. R. Mcdonald, and S. J. Galloway, "GA/SA-based hybrid techniques for the scheduling of generator maintenance in power systems," in IEEE Conference on Evolutionary Computation, 2000, pp. 567-574.

[104] F. Glover, E. Taillard, and D. de Werra, "A user's guide to tabu search," Ann. Oper. Res., vol. 41, pp. 3-28, 1993.

[105] F. Glover and M. Laguna, Tabu search. MA: Kluwer Academic Publishers, 1997.

[106] T. Sawa, T. Furukawa, M. Nomoto, T. Nagasawa, T. Sasaki, K. Deno, and T. Maekawa, "Automatic scheduling method using tabu search for maintenance outage tasks of transmission and substation system with network constraints," IEEE Power Eng. Soc. 1999 Winter Meet., vol. 2, pp. 895-900, 1999.

[107] I. El-Amin, S. Duffuaa, and M. Abbas, "A tabu search algorithm for maintenance scheduling of generating units," Electr. Power Syst. Res., vol. 54, pp. 91-99, 2000.

[108] M. Gopalakrishnan, S. Mohan, and Z. He, "A tabu search heuristic for preventive maintenance scheduling," Comput. Ind. Eng., vol. 40, no. 1-2, pp. 149-160, Jun. 2001.

[109] H. Kim, Y. Hayashi, and K. Nara, "An algorithm for thermal unit maintenance scheduling through combined use of GA, SA and TS," IEEE Trans. Power Syst., vol. 12, no. 1, pp. 329-335, 1997.

[110] E. Borgonovo, M. Marseguerra, and E. Zio, "A Monte Carlo methodological approach to plant availability modeling with maintenance, aging and obsolescence," Reliab. Eng. Syst. Saf., vol. 67, no. 1, pp. 61-73, Jan. 2000.

[111] a. Crespo Marquez, J. N. D. Gupta, and J. P. Ignizio, "Improving preventive maintenance scheduling in semiconductor fabrication facilities," Prod. Plan. Control, vol. 17, no. 7, pp. 742-754, Oct. 2006.

[112] R. Bris, E. Châtelet, and F. Yalaoui, "New method to minimize the preventive maintenance cost of series-parallel systems," Reliab. Eng. Syst. Saf., vol. 82, no. 3, pp. 247-255, Dec. 2003.

[113] M. Bevilacqua and M. Braglia, "The analytic hierarchy process applied to maintenance strategy selection," Reliab. Eng. Syst. Saf., vol. 70, no. 1, pp. 71-83, Oct. 2000.

[114] V. S. Deshpande and J. P. Modak, "Application of RCM for safety considerations in a steel plant," Reliab. Eng. Syst. Saf., vol. 78, no. 3, pp. 325-334, Dec. 2002.

[115] V. S. Deshpande and J. P. Modak, "Application of RCM to a medium scale industry," Reliab. Eng. Syst. Saf., vol. 77, no. 1, pp. 31-43, Jul. 2002.

[116] C. Cassady and E. Kutanoglu, "Minimizing job tardiness using integrated preventive maintenance planning and production scheduling," IIE Trans., vol. 35, 
pp. 503-513, 2003.

[117] F. Benbouzid, C. Varnier, and N. Zerhouni, "Resolution of joint maintenance/production scheduling by sequential and integrated strategies," Artif. Neural Nets Probl. Solving Methods, vol. 2687/2003, pp. 782-789, 2003.

[118] C. R. Cassady and E. Kutanoglu, "Integrating Preventive Maintenance Planning and Production Scheduling for a Single Machine," IEEE Trans. Reliab., vol. 54, no. 2, pp. 304-309, Jun. 2005.

[119] F. Benbouzid-Sitayeb, I. Ammi, C. Varnier, and N. Zerhouni, “Applying Ant Colony Optimization for the Joint Production and Preventive Maintenance Scheduling Problem in the Flowshop Sequencing Problem," 2008 3rd Int. Conf. Inf. Commun. Technol. From Theory to Appl., pp. 1-6, Apr. 2008.

[120] M. Dorigo, V. Maniezzo, and A. Colorni, "Ant system: optimization by a colony of cooperating agents.," IEEE Trans. Syst. Man, Cybern. B, vol. 26, no. 1, pp. 113, Jan. 1996.

[121] A. Berrichi, L. Amodeo, F. Yalaoui, E. Châtelet, and M. Mezghiche, "Bi-objective optimization algorithms for joint production and maintenance scheduling: application to the parallel machine problem," J. Intell. Manuf., vol. 20, no. 4, pp. 389-400, Jun. 2009.

[122] M. Ben-Daya, "Integrated production maintenance and quality model for imperfect processes," IIE Trans., vol. 31, no. 6, pp. 491-501, Jun. 1999.

[123] E. H. Aghezzaf, M. a. Jamali, and D. Ait-Kadi, "An integrated production and preventive maintenance planning model," Eur. J. Oper. Res., vol. 181, no. 2, pp. 679-685, Sep. 2007.

[124] A. Chelbi, N. Rezg, and M. Radhoui, "Simultaneous determination of production lot size and preventive maintenance schedule for unreliable production system," $J$. Qual. Maint. Eng., vol. 14, no. 2, pp. 161-176, 2008.

[125] S. El-Ferik, "Economic production lot-sizing for an unreliable machine under imperfect age-based maintenance policy," Eur. J. Oper. Res., vol. 186, no. 1, pp. 150-163, Apr. 2008.

[126] G.-L. Liao, Y. H. Chen, and S.-H. Sheu, "Optimal economic production quantity policy for imperfect process with imperfect repair and maintenance," Eur. J. Oper. Res., vol. 195, no. 2, pp. 348-357, Jun. 2009.

[127] M. Ben Ali, M. Sassi, M. Gossa, and Y. Harrath, "Simultaneous scheduling of production and maintenance tasks in the job shop," Int. J. Prod. Res., vol. 49, no. 13, pp. 3891-3918, Jul. 2011.

[128] Y.-L. Jin, Z.-H. Jiang, and W.-R. Hou, "Integrating flexible-interval preventive maintenance planning with production scheduling," Int. J. Comput. Integr. Manuf., vol. 22, no. 12, pp. 1089-1101, Dec. 2009.

[129] D. A. Coley, An Introduction to Genetic Algorithms for Scientists and Engineers, 
vol. 174, no. 4480. MA: World Scientific Publishing Co.Pte. Ltd., 1999.

[130] S. M. R. Iravani and I. Duenyas, "Integrated maintenance and production control of a deteriorating production system," IIE Trans., vol. 34, no. 5, pp. 423-435, May 2002.

[131] X. Yao, X. Xie, M. C. Fu, and S. I. Marcus, "Optimal joint preventive maintenance and production policies," Nav. Res. Logist., vol. 52, no. 7, pp. 668-681, Oct. 2005.

[132] B. Kazaz and T. W. Sloan, "Production policies under deteriorating process conditions," IIE Trans., vol. 40, no. 3, pp. 187-205, Jan. 2008.

[133] Y. Cai, J. Hasenbein, E. Kutanoglu, and M. Liao, "Single-machine Multiple-recipe Predictive Maintenance," Probab. Eng. Informational Sci., vol. 27, no. 2, pp. 209235, 2013.

[134] D. L. Kaufman and M. E. Lewis, "Machine maintenance with workload considerations," Nav. Res. Logist., vol. 54, no. 7, pp. 750-766, Oct. 2007.

[135] J. Zhou, D. Djurdjanovic, J. Ivy, and J. Ni, "Integrated load-allocation and condition-based maintenance policy in a multi-unit load -sharing deteriorating system," in Proceedings of the 61st Meeting of the Society for Machine Failure Prevention Technology, 2007, pp. 215-228.

[136] T. W. Sloan and J. G. Shanthikumar, "Combined Production and Maintenance Scheduling for a Multiple-Product, Single- Machine Production System," Prod. Oper. Manag., vol. 9, no. 4, pp. 379-399, Jan. 2000.

[137] T. Sloan, "A periodic review production and maintenance model with random demand, deteriorating equipment, and binomial yield," J. Oper. Res. Soc., vol. 55 , no. 6, pp. 647-656, 2004.

[138] T. W. Sloan and J. G. Shanthikumar, "Using in-line equipment condition and yield information for maintenance scheduling and dispatching in semiconductor wafer fabs," IIE Trans., vol. 34, no. 2, pp. 191-209, Feb. 2002.

[139] D. J. D. Wijnmalen and J. a. M. Hontelez, "Coordinated condition-based repair strategies for components of a multi-component maintenance system with discounts,” Eur. J. Oper. Res., vol. 98, no. 1, pp. 52-63, Apr. 1997.

[140] J. Banks, “Getting started with Automod," 2000.

[141] F. Geyik and I. Cedimoglu, "The strategies and parameters of tabu search for jobshop scheduling," J. Intell. Manuf., vol. 15, pp. 439-448, 2004.

[142] M. E. Cholette and D. Djurdjanovic, "Degradation Modeling and Monitoring of Machines Using Operation-Specific Hidden Markov Models," IIE Trans., vol. 46, no. 10, pp. 1107-1123, 2014.

[143] D. Zhang, A. D. Bailey III, and D. Djurdjanovic, "Bayesian Identification of Hidden Markov Models and Their Use for Condition-Based Monitoring," Accept. by IEEE Trans. Reliab., p. Paper No. TR-2015-183, 2015. 
[144] R. Howard, Dynamic Programming and Markov Processes. Cambridge, MA: The M.I.T. Press, 1960.

[145] R. Kaplan, "Optimal investigation strategies with imperfect information," $J$. Account. Res., vol. 7, no. 1, pp. 32-43, 1969.

[146] J. Hughes, "Optimal internal audit timing," Account. Rev., vol. 52, no. 1, pp. 56$68,1977$.

[147] W. Karush and R. Dear, "Optimal strategy for item presentation in a learning process,” Manage. Sci., vol. 13, no. 11, pp. 773-786, 1967.

[148] T. Nakai, "Properties of a job search problem on a partially observable Markov chain in a dynamic economy," Comput. Math. with Appl., vol. 51, no. 2, pp. 189198, Jan. 2006.

[149] M. Girshick and H. Rubin, "A Bayes approach to a quality control model," Ann. Math. Stat., vol. 23, pp. 114-125, 1952.

[150] H. Taylor, "Markovian sequential replacement processes," Ann. Math. Stat., vol. 36, no. 6, pp. 1677-1694, 1965.

[151] S. Ross, "Quality control under Markovian deterioration," Manage. Sci., vol. 17, no. 9, pp. 587-596, 1971.

[152] J. S. Ivy and H. B. Nembhard, "A Modeling Approach to Maintenance Decisions Using Statistical Quality Control and Optimization," Qual. Reliab. Eng. Int., vol. 21, no. 4, pp. 355-366, Jun. 2005.

[153] R. Smallwood and E. Sondik, "The optimal control of partially observable Markov processes over a finite horizon," Oper. Res., vol. 21, no. 5, pp. 1071-1088, 1973.

[154] C. White, "Optimal control-limit strategies for a partially observed replacement problem†,” Int. J. Syst. Sci., vol. 10, pp. 321-331, 1979.

[155] W. Lovejoy, "Some monotonicity results for partially observed Markov decision processes," Oper. Res., vol. 35, no. 5, pp. 736-743, 1987.

[156] A. H. Elwany, N. Z. Gebraeel, and L. M. Maillart, "Structured Replacement Policies for Components with Complex Degradation Processes and Dedicated Sensors," Oper. Res., vol. 59, no. 3, pp. 684-695, Jul. 2011.

[157] E. Byon, L. Ntaimo, and Y. Ding, "Optimal Maintenance Strategies for Wind Turbine Systems Under Stochastic Weather Conditions," IEEE Trans. Reliab., vol. 59, no. 2, pp. 393-404, Jun. 2010.

[158] L. Jin, K. Miyawaki, and K. Suzuki, "Optimal Maintenance Policy For Partially Observable Markovian Deteriorating System Subject To A Controllable Varying Environment," J. Reliab. Eng. Assoc. Japan, vol. 35, no. 3, pp. 177-187, 2013.

[159] G. E. Monahan, "A Survey of Patially Observable Markov Decision Processes: Theory, Models, and Algorithms," Manage. Sci., vol. 28, no. 1, pp. 1-16, 1982.

[160] T. Sawa, T. Furukawa, M. Nomoto, T. Nagasawa, T. Sasaki, K. Deno, and T. 
Maekawa, "Automatic scheduling method using tabu search for maintenance outage tasks of transmission and substation system with network constraints," IEEE Power Eng. Soc. 1999 Winter Meet., vol. 2, pp. 895-900, 1999.

[161] J. K. Satia and R. E. . Lave Jr, "Markovian Decision Processes with Uncertain Transition Probabilities," Oper. Res., vol. 21, no. 3, pp. 728-740, 1973.

[162] A. Nilim and L. El Ghaoui, "Robust Control of Markov Decision Processes with Uncertain Transition Matrices," Oper. Res., vol. 53, no. 5, pp. 780-798, 2005.

[163] L. F. Bertuccelli and J. P. How, "Robust Markov Decision Processes Using Sigma Point Sampling," in 2008 American Control Conference, 2008, pp. 5003-5008.

[164] A. Atrash and J. Pineau, "A Bayesian Method for Learning POMDP Observation Parameters for Robot Interaction Management Systems," in The International POMDP Practitioners Workshop, 2010.

[165] L. R. Rabiner, "A Tutorial on Hidden Markov Models and Selected Applications in Speech Recognition," Proc. IEEE, vol. 77, no. 2, pp. 257-286, 1989.

[166] L. E. Baum and J. A. Eagon, "An inequality with applications to statistical estimation for probabilistic functions of Markov processes and to a model for ecology," Bull. Am. Math. Soc., vol. 73, pp. 360-363, 1967.

[167] L. E. Baum and G. R. Sell, "Growth transformations for functions on manifolds," Pacific J. Math., vol. 27, no. 2, pp. 211-227, 1968.

[168] G. D. Forney, “The Viterbi algorithm,” Proc. IEEE, vol. 61, pp. 268-278, 1973.

[169] A. P. Dempster, N. M. Laird, and D. B. Rubin, "Maximum Likelihood from Incomplete Data via the EM Algorithm," J. R. Stat. Soc., vol. 39, no. 1, pp. 1-38, 1977.

[170] F. Yang, C. Zang, and G. Bai, "A Novel Genetic Algorithm Based on Tabu Search for HMM Optimization," in Fourth International Conference on Natural Computation, 2008, no. 1, pp. 57-61.

[171] M. Slimane, G. Venturini, J.-P. A. de Beauville, T. Brouard, and A. Brandeau, "Optimizing Hidden Markov Models with a Genetic Algorithm," in Artificial Evolution, volume 1063 of Lecture Notes in Computer Science, Springer, 1996, pp. 384-396.

[172] J.-S. Lee and C. H. Park, "Training Hidden Markov Models by Hybrid Simulated Annealing for Visual Speech Recognition," in 2006 IEEE International Conference on Systems, Man, and Cybernetics, 2006, pp. 198-202.

[173] C. Champion and S. M. Houghton, "Application of continuous state Hidden Markov Models to a classical problem in speech recognition," Comput. Speech Lang., vol. 36, pp. 347-364, 2016.

[174] M. Gales and S. Young, "The Application of Hidden Markov Models in Speech Recognition," Found. Trends Signal Process., vol. 1, no. 3, pp. 195-304, 2007. 
[175] M. Durvey and D. Satyarthi, "A Review Paper on Statistical Methods," Int. J. Emerg. Trends Technol. Comput. Sci., vol. 4, no. 5, pp. 33-39, 2015.

[176] S. R. Eddy, "Hidden Markov models," Curr. Opin. Struct. Biol., vol. 6, pp. 361365, 1996.

[177] C. Bunks and D. McCarthy, "Condition-based maintenance of machines using hidden Markov models," Mech. Syst. Signal Process., vol. 14, no. 4, pp. 597-612, 2000 .

[178] L. Atlas, M. Ostendorf, and G. D. Bernard, "Hidden Markov models for monitoring machining tool-wear," in 2000 IEEE International Conference on Acoustics, Speech and Signal Processing, 2000, pp. 3887-3890.

[179] H. M. Ertunc and C. Oysu, "Drill wear monitoring using cutting force signals," Mechatronics, vol. 14, pp. 533-548, 2004.

[180] A. G. Rehorn, J. Jiang, and P. E. Orban, "State-of-the-art methods and results in tool condition monitoring: a review," Int. J. Adv. Manuf. Technol., vol. 26, pp. 693-710, 2005.

[181] Z. Li, Z. Wu, Y. He, and C. Fulei, "Hidden Markov model-based fault diagnostics method in speed-up and speed-down process for rotating machinery," Mech. Syst. Signal Process., vol. 19, pp. 329-339, 2005.

[182] V. Purushotham, S. Narayanan, and S. A. N. Prasad, "Multi-fault diagnosis of rolling bearing elements using wavelet analysis and hidden Markov model based fault recognition," NDT\&E Int., vol. 38, pp. 654-664, 2005.

[183] Y. Peng, M. Dong, and M. J. Zuo, "Current status of machine prognostics in condition-based maintenance : a review," Int. J. Adv. Manuf. Technol., vol. 50, pp. 297-313, 2010.

[184] M. E. Cholette, "Performance Monitoring and Fault-Tolerant Control of Complex Systems with Variable Operating Conditions," Ph.D. Dissertation, The University of Texas at Austin, 2012.

[185] T. Sloan, "Simultaneous Determination of Production and Maintenance Schedules Using In-Line Equipment Condition and Yield Information," Nav. Res. Logist., vol. 55, no. 2, pp. 116-129, 2008.

[186] S. Lee and Y.-C. Tien, "In Situ and Real-Time Monitoring of Plasma Process Chamber Component Qualities and Predictive Controlling of Wafer Yields," IEEE Trans. Semicond. Manuf., vol. 19, no. 4, pp. 432-436, 2006. 